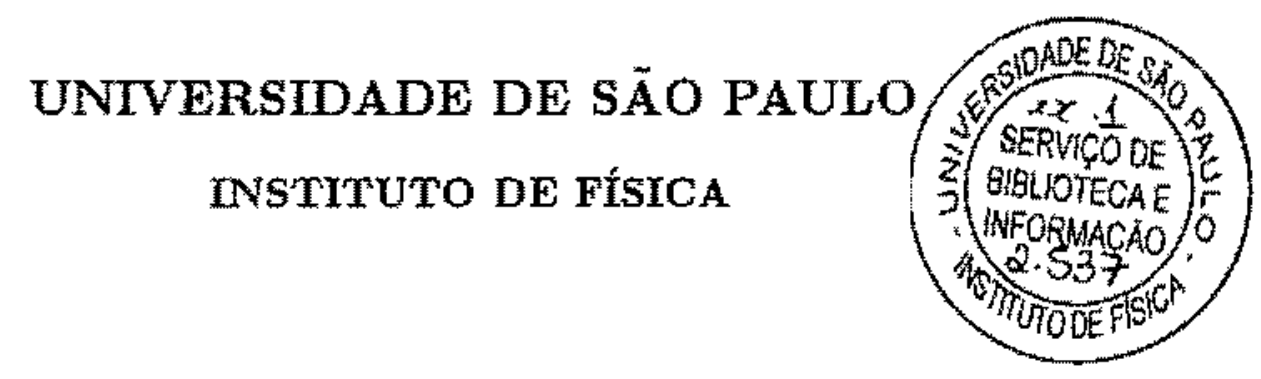

\title{
Teoria Cinética de Gases Poliatômicos: Efeito Senftleben-Beenakker e Equações Linearizadas de Burnett
}

\author{
ALCIONE SHVA FERNANDES
}

Tese apresentada ao Instituto de Fisica da Universidade de Săo Paulo como parte dos requisitos necessários à obtençẫo do título de Doutor em Ciencias.

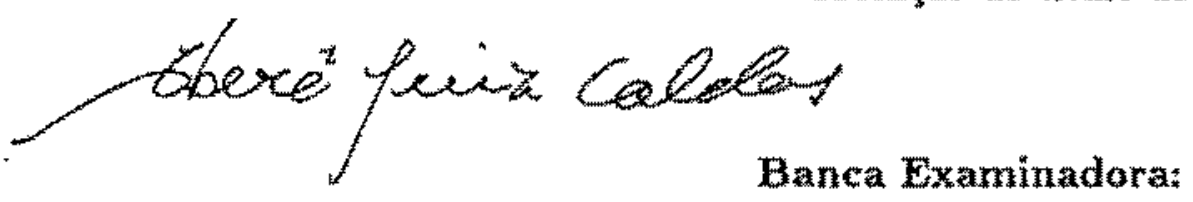

Prot, Dr. Giberto Medetros Kremer (UFPR)

Prof. Dr. 1-Shin List (UFRJ)

Prof. Dr, Jose Gatuto Ramos (UNICAMP)

Profa Dra. Tanta Tomé Mantins de Casto (IFUSP)

Prof. Dr. Valdar Binduata (IFUSP)

Orientador: Prof: Dt. Lbere Luiz Caldas

Co-Orientador: Prof. Dr. Giberto Medeiros Krewer

Sẫo Paulo

1996
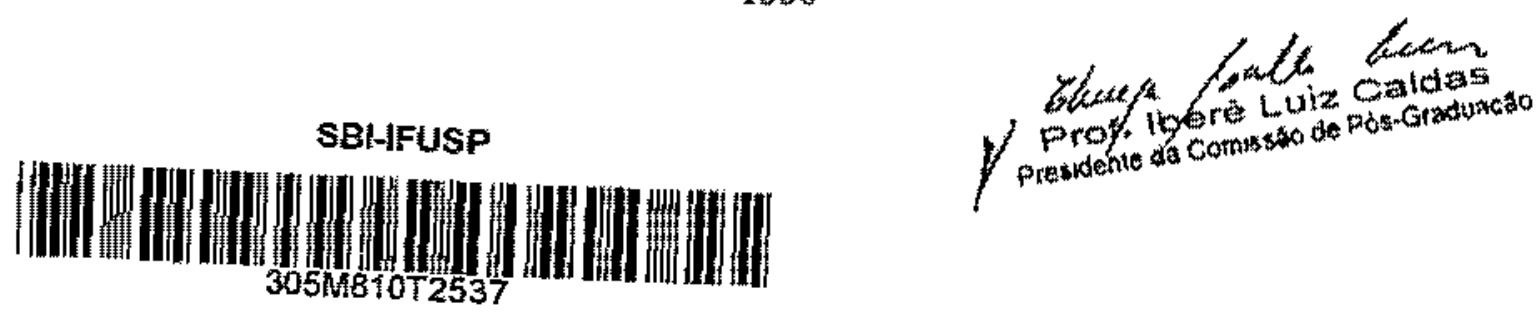


$$
\begin{gathered}
530.13 \\
F 3631 \\
= \\
2 x .1
\end{gathered}
$$

\section{FICHA CATALOGRÁFICA}

Preparada pelo Serviço de Biblioteca e Informação do Instituto de Física da Universidade de São Paulo

\section{Fernandes, Alcione Silva}

Teoria Cinética de Gases Poliatốmicos: Efeito Senftleben - Beenakker e Equaçöes Linearizadas de Burnett.

Tese (Doutorado) Universidade de São Paulo. Instituto de Fisica. Departamento de Física Aplicada.

Área de Concentração: Física Nuclear

Orientador: Prof. Dr. lberế Luiz Caldas

Unitermos: 1. Gases Poliatômicos; 2. Teoria Cinética de Gases; 3. Coeficientes de Transporte; 4. Mecânica Estatistica. 
A menória de mets pais Alziro Alzira.

À minha esposa Nelma $e$ às minhas filtras Maria Luiza, Maria Alice e Maria Angela. 
"Ai, experimentei o meu estilo, como é que estarík. Me aqratou. De certo que eu amava a lingua. Apenas năo a amo como a mäe severa, mas como a bela amante companheira. O que eu gostaria de poder fazer (näto o que faz, João Condét) seria aplicar, no kaso, a minha interpretaçäo de uns versos de Paul Ehtard: ... "o peize avança nägua, como um dedo numa luva" ... Um ideal. precisäo, micromilimétrica. E riqueza, oh! riqueza... Pelo menos, impiedoso, horror ao lugar comum; que as chapas säo pedaços de came corrompida, säo pecados contra o Espírito Santo, são taperas no territónio do idioma.

Mas, atuda haveria mats, se possível (sonhar é fäcil, Joäo Condé, realizar é que são elas...): alén los zstados líguidos e sólidos, porque näo tentar trabalhar a língua

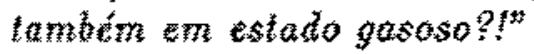

(trecho de carla de Gumarases Rosa a Joäo Condt, rezelando segredos de Sagarana) 


\section{Agradecimentos}

Na realização desta tese recebi o apoio de várias pessoas; em especial, agradeço:

an men prezado orientador professor Gilberto Medeiros Kremer pela orientaçüo dedicada ao longo do desenvolvimento desta tese;

ao professor Iberê Luiz Caldas pelo estímulo e atençẫo os quais tornaram possível a realização deste trabalho;

ao professor Silvio Roberto de Azevedo Salinas pela atenciosa orientaçäo académica no meu primeiro ano de USP;

à professora Suzana Reinecke pelo valioso auxilio computacional na realizaçấo de vários cálculos algébricos destáa tese;

ao professor Wilson Marques Jr. pela leitura paciente deste trabalho e valiosas sugestōes;

aos professores Márcio Henrique Franco Bettega, Ricardo Luiz Viana e Jair Lucinda pelo estimulo e apoio;

aos professores Cláudio Henrique Lepienski, Mauro Gomes Rodbard, Lauro Samojden e Felix Sharipov, colegas do grupo de Termodinâmica Fenomenológica estatística, pela convivéncia amigavel;

à minha filha Maria Angela pelo entusiasmo com que realizou alguns desenhos desta tese. 


\section{Resumo}

Neste trabalho desenvolvemos métodos de solução para gases poliatômicos clâssicos sob o ponto de vista da teoria cinética com base na equação de Boltzmann para moléculas esféricas com energia rotacional. Dois temas sâo abordados:

(i) corn o método combinado de Chapman-Enskog e Grad e o emprego de teoremas de representaçâa apresentamos uma forma sistemática para obter os coeficientes de transporte de gases poliatômicos submetidos a campos magnéticos extenos constantes (efeito SenftlebenBeenakker);

(ii) obtemos equaçōes linearizadas de Burnett, correspondentes a gradientes de segunda ordem dos campos de densidade, velocidade e temperatura, de duas maneiras distintas: (a) através da teoria cinética com o emprego do método de Chapman-Enskog e (b) segundo uma teoria fenomenológica com base na termodinàmica estendida. 


\section{Abstract}

We develop methods of solution for classical rarefied polyatomic gases with spherical molecules having rotational energy through a kinetic theory based on the Boltzmann equation. Two subjects are considered:

(i) by the use of the combined method of Chapman-Euskog and Grad and representation theorems we show a systematical way to get the transport coefficients for polyatomic gases subjected to external constant magnetic fields (Senftleben*Beenakker effect);

(ii) we obtain lizearized Burnett equations that correspond to gradients of second order of the basic fields of density, velocity and temperature through: (a) a kinetic theory by using the Chapman-Enskog method and (b) a phenomenological theory based on extended thermody. namies. 


\section{Conteúdo}

Agradecimentos

Resumo

$\begin{array}{ll}\text { Abstract } & \text { iii }\end{array}$

Lista de Figuras $\quad$ vii

Lista de Tabelas

$\begin{array}{ll}\text { Introdução } & 1\end{array}$

1 Fundamentos da Teoria Cinética de Gases Poliatômicos 8

1.1 Introduçầo $\ldots \ldots \ldots \ldots \ldots$

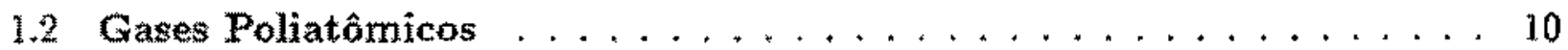

1.3 Gases Poliatômicos de Moléculas Rugosas . . . . . . . . . . . . . 14

1.3.1 Dinâmica da Colisấo Binăria ............... 15

1.3 .2 Equação de Boltzmann $\ldots \ldots \ldots \ldots$

1.3.3 A Equaçăo de Transporte es Invariantes de Soma . . . . . . . 23

2 Descriçẫo Macroscópica de um Gás Ideal Poliatônico 27

2.1 Introdução $\ldots \ldots \ldots \ldots \ldots \ldots \ldots \ldots$

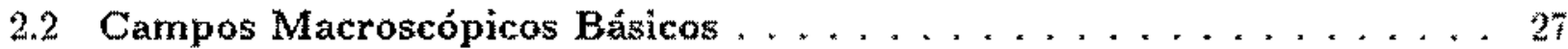

2.3 Equaçöes de Balanço para Campos Macroscópicos . . . . . . . . . . 29

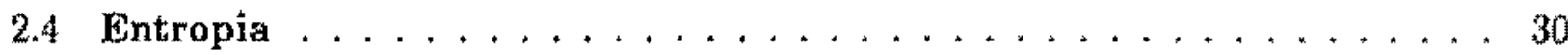

2.5 Função de Distribuição para o Estado de Equilíbrio . . . . . . . . . . 32

2.6 Descriçăo Macroscópica do Estado de Equilíbrio . . . . . . . . . . 34

2.7 Equaçöes de Balanço para o Estado de Equilíbrio . . . . . . . . . . . 35

3 Métodos de Solução da Equaçäo de Boltzmann 37

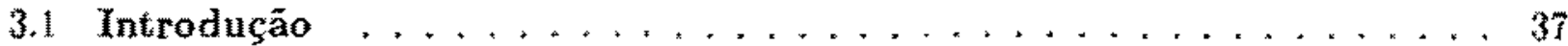


3.2 O Método de Chapman-Enskog . . . . . . . . . . . 37

3.3 O Método dos Momentos de Grad . . . . . . . . . . . . . 46

3.4 O Método Combinado de Chapman-Enskog e Grad ......... 54

4 Gases Poliatỗmicos em Campos Magnéticos Externos $\quad 57$

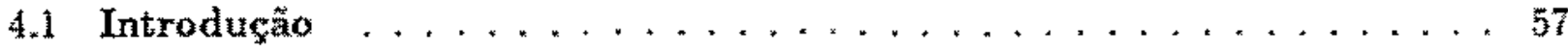

4.2 Campos Básicos $\ldots \ldots \ldots \ldots \ldots \ldots \ldots \ldots \ldots \ldots \ldots$

4.3 A Função de Distribuição de Grad . . . . . . . . . . . 64

4.4 O Método Combinado de Chapman-Enskog e Grad . . . . . . . . 66

4.5 Coeficientes de Condutividade Térmica . . . . . . . . . . . . 69

4.5.1 A Solução de Pidduck para o Coeficiente de Condutividade Térmica . . . . . . . . . . . . . . . . . . 71

4.5.2 Campo $H_{l}^{\prime \prime}(\mathrm{x}, t)$ e Coeficientes de Condutividade Térmica ... 72

4.5.3 Campo $H_{\langle i j\rangle k}(x, t)$ e Coeficientes de Condutividade Térmica . 78

4.6 Coeficientes de Viscosidade para Gases Poliatômicos . . . . . . . . 92

4.6.1 Determinação dos Coeficientes de Viscosidade Cisalhante . . . 94

4.6 .2 Coeficiente de Viscosidade Volumétrica . . . . . . . . . . 107

$4 . \overline{7}$ Conclusōes . . . . . . . . . . . . . . . . . . 107

5 Equaçōes Linearizadas de Burnett 111

5.1 Introduçä̃o $\ldots \ldots \ldots \ldots \ldots \ldots \ldots \ldots \ldots \ldots \ldots \ldots \ldots$

5.2 Teoria Cinética . . . . . . . . . . . . . . . . . . . 112

5.2.1 Segunda Aproximação para a Funçãa de Distribuiçăo . . . . 112

5.2 .2 Terceira Aproximaçäo para a Funçâ̄o de Distribuição . . . . 114

5.2.3 Coeficientes de Transporte para as Equaçöes Linearizadas de Burnett . . . . . . . . . . . . . . . . . . . . 122

5.3 Teoria Fenomenológica . . . . . . . . . . . . . . . . 125

5.3 .1 Resultados de uma Teoria Termodinâmica Estendida . . . . . 125

5.3.2 Comparaçäo Entre Grandezas das Duas Teorias . . . . . . . 130

5.3 .3 Equaçōes de Balanço Linearizadas . . . . . . . . . . . . 131

5.3.4 Iteração Maxwelliana e as Equações Linearizadas de Burnett . . 134

5.3 .5 Densidade e Fluxo de Entropia . . . . . . . . . . . 138

5.3.6 As Temperaturas $\vartheta$ e $T$ como Variâveis Termodinămicas . . . . 139

5.3.7 Coeficientes de Transporte das Equações Linearizadas de Burnett . . . . . . . . . . . . . . . . . . . 140

5.4 Conclusồes . . . . . . . . . . . . . . . . . . 143 
A. Integrais em Teoria Cinética

B Representaçäes Tensoriais

150

B.I Representação para o Tensor $H_{<i j} \ldots \ldots \ldots \ldots \ldots \ldots$

B.2 Representação para um Tensor de Quarta Ordem .......... 152

B.3 Inversão de um Tensor de Segunda Ordem ............... la

B.4 Inversão de um Tensor de Quarta Ordem ..............154

$\begin{array}{ll}\text { C Integraçäo de uma Integral de Colisäo } & 156\end{array}$

D Polinômios de Sonine $\quad 159$

$\begin{array}{ll}\text { E Condiçăo de Salto para Campos } & 161\end{array}$

$\begin{array}{lr}\text { Bibliografia } & 164\end{array}$ 


\section{Lista de Figuras}

1.1 Valores experimentais para o calor especifico $c_{y}$ para o gás $\mathrm{H}_{2} \ldots \ldots \ldots$

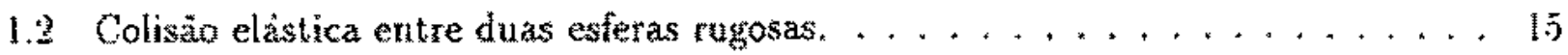

1,3 Colisó direta e inversa de moletulas no modelo de esferas lisas. . . . . . . . 18

1.4 Esfera de infuência numa colisăo binatia. . . . . . . . . . . . . 21

4.1 Os coeficientes de condutividade térmica: (a) paralelo, (b) perpendicular e (c)

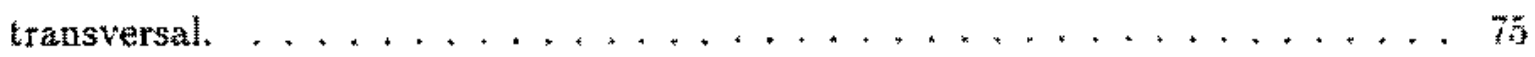

4.2 Varianoes relativas do coeficiente de condubividade térmica perpendicular . . . . if

4.3 Variaçöes relativas do coeficientu de condutividade témica transversal . . . . . T

4 Variaçòes relativas para o coeficiente de condutividade térnica paralelo. . . . . 90

4.5 Variaçoes relativas para o coeficiente de condutividade de térmica perpendicular. 91

4.6 Variaçoes telativas para o coeficiente de condutividade térmica transversal. . . 91

4.7 Variaçato do parâmetro $R$ em funçào do campo magnético de saturaçäo . . . . .93

4.8 Variações relativas dos coeficientes te viscosidade cisalhante. . . . . . . . 104

4.9 Dispositivo empregado na medila dos coeficientes de viscosidade. . . . . . I05

4. 10 Arranjo esquemático utilisado na determinaçâo dos coeficientes de viscosidade $\eta_{3}$ e $\eta_{5} \ldots \ldots \ldots \ldots \ldots \ldots \ldots$

E.1 Superficie singular para um volume de fuido $\ldots \ldots \ldots \ldots$ 


\section{Lista de Tabelas}

1.1 Temperaturas caranterísticas para alguns zases poliatómicos. . . . . . . . II

1.2 Valores caracteristicos para alguns gases poliatomicos a temperatura de $273 \mathrm{~K} \mathrm{e}$ pressăo de $1,01 \times 10^{5} \mathrm{P} \ldots \ldots \ldots \ldots \ldots \ldots$

1.3 Valores experimentais e teóricos para o numero de Eucken. . . . . . . 13

4.1 Valores experimentais e teóticos para o parâmetro $\mathrm{R} \ldots \ldots \ldots$

4.2 Comportentes do tensor pressäo $\ldots \ldots \ldots \ldots \ldots$

5.1 Comparaça entre as duas teorias. . . . . . . . . . . . . 130

E.1 Densidades, fuxos e produçóes para a grandeza $\Psi \ldots \ldots \ldots$ 


\section{Introdução}

A mecânica estatística pode ser dividida em duas grandes áreas de interesse: a que trata de sistemas en equilbrio e a que focaliza os sistemas fora do equilintio. A teotia cinética de gases Na teoria cinética clássica os gases sảo considerados como constituídos de um número muito grande partículas com mowimentos descritos pelas leis da mecanica clássica. O conceito de livte caminho médio para uma partícula, introduzido por Clausius em 1858 , pode ser considerado o início da teoria cinética moderna.

Em 1859, Maxwell [1] derivou fómulas para os coeficientes de transporte de gases (viscosidade. condutividade térnica e difusâo) empregando a idéia de livre carninho médio de Clausius; alem disso introduziu na teoria cinética a definição estatística de funçăo de distribuiçăo de velocidades obtendo a sua forma para un gás em equilíbrio (distribuiçào maxwelliana de welocidades).

Em 1867, Maxwell [2] propờ uma equaça geral de transporte para uma grandeza macroscópica qualquer, definida como o valor médio de uma grandeza microscópica associada às partículas do gás. A equação geral de transporte relaciona a taxa de mudança temporal da grandeza macroscópica com o movimento das partículas, as colisóces entre elas e açấo de forças externas sobre as partículas. No entanto Maxwell só conseguiu obter os coeficientes de transporte considerando que as partículas se repeliam segundo uma força inversamente proporctonal à quinta potência da distância entre elas (moléculas maxwellianas).

No ano de 1872, Boltzmann [3] propôs uma equação integrondiferencial que descreve a

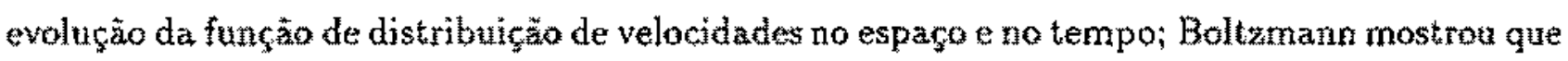
as fórmulas obtidas por Maxwell para os coeficientes de transporte com o modelo de moléculas maxwellianas podiam ser obtidas como solução da equação integrom diferencial por de formulada (equaçào de Bolkzmann). Neste mesmo artigo, Boltzmann estabeleceu o chamado teorema H que estabelece a irreversbllidade natural dos processos fisicos e mostrou como as colisões moleculares tendem a aumentar a entropia do gás (qualquer função inicial de distribuiçäo de velocidades e posições a as moléculas evolui para um estado de equilbrio descrito pela funçäo de distribuiçào maxwelliana). As bases formais da teorta cinética foram ampliadas com a generall. zaçăo do teorema $\mathrm{H}$ para um gás submetido a um campo de força conservativo, por Boltzmann [4] L Lorentz [5].

Em 1912, o matemático David Hibert [6] indicou como obter soluçöes aproximadas da equaçăo de Boltzmann atraves de expansöes efn serte num parametro inversamente proporciutal 


\section{à densidade do gás.}

Nos anos de 1916 e 1917, de maneiras independentes, Chapman [7] e Enskog [8] obtiveram soluçós aproximadas para a equaçäo de Boltzmann. Os resultados obtidos foram idênticos mas os mêtodos utilizados eram completamente diferentes. Chapman estendeu o método de Maxwell utilizando a equação de transporte enquanto Enskog apresentou uma técnica sistemática de expansão generalizando o método sugerido por Hibert. Em 1939, Chapman e Cowling [9] publicaram o livro "The Mathematical Theory of Non-Uniform Gases" baseado nos dois métodos; este novo método passou a ser denominado de método de Chapman-Enskog.

Una extensäo dos resultados de Chapman e Enskog fot apresentada por Burnett [10] que empregou uma aproximação de segunda ordem para a funçäo de distribuiçăo. As equaçöes obtidas țesta forma são atualmente conhecidas como equaçoes de Burnett.

Num artigo de 1949. Grad [11] apresentou um método sistemático de solução para a equaçào de Boltzmann através da expansão da soluçŭo numa série de polinômios ortogonais o qual é hoje conhecito como método de Grad.

Para estender a aplicação da equaçăo de Boltzmann aos gases poliatômicos encontramos algumas dificuldades. Em primeiro hgar, porque as moleculas poliatömicas apresentam formas variadas de modo que os potenciais intermoleculares näo sẫo esfericamente simétricos; em segundo lugar, porque as moléculas poliatómicas apresentam graus de liberdade internos (rotaçăo, vibração e excitaçäo eletrồica) com os quais energia pode ser associada.

Uma tentativa para estender a equaçăo de Boltzmann aos gases poliztôrnicos foi realizada. na década de 1940, por Wang-Chang e Uhlenbeck e, independentemente, por de Boer [12]. Embora nào rigorosa exta teoria semiclássica apontou na direça de una teoria mais completa. Uma teoria quântica para gases poliatơnicos foi apresentada por Waldmann [13] e Snider [14] e posteriormente aplicada por MeCourt e Snider [15] considerando a energia de rotaçăo das moléculas do gás.

$O$ estudo dos gases poliatomicos apresentou grande impulso a partir do início da década de 1960 quando Beenakker e colaboradores [16] empreenderam um estudo sistemático, teórico e exparimental de gases poliatơnicos submetidos a campos magnéticos externos (efeito SenfulebenBeenakker), o qual te tema de intensas pesquisas ainda nos djas de hoje.

Neste trabalho desenvolvemos dois temas tratando de gase poliatömicos: o efaito SenftlebenBeenakker e as equaçôs linearizadas de Burnett. No capítulo 1 apresentamos a dinàmica da colisăo binária para o modelo de Bryan [17], o qual é empregado ao longo deste trabalbo.

No eapitulo 2 discutimos as primcipais caracteristicas para o extado de equilibrio de um gás poliatomico rarefeito bem como apresentamos a funçào de distribuiçào de velocidades para 0 estado da equilibrio.

No capitulo 3 mostramos os métodos tradicionais de soluçăo da equaçäo de Bolizmann ou 
seja, o método de Chapman-Enskog e o método de Grad. Além destes, introduzimos o método combinado de Chapman-Enskog Grad, desenvolvido por Bezerra Jr, Reinecke e Kremer [18]. Este último método apresentou aspectos vantajosos em relação relaçäo ans métodos tradicionais na obtenção de coeficientes de transporte para gases monoatômicos [18] e găser ionizados [19].

No capítulo 4 empregamos o método combinado de Chapman-Enskog Grad com objetivo de determinar as mudanças que ocorrem nos coeficientes de transporte para um gâs poliatômico sob um campo magnético constante, denominado efeito Senftleben-Beenakker, 0 gual descrevemos a seguir.

\section{Efeito Senftleben-Beenakker}

Em 1930 foi observado por Senfteben que os valores do coeficiente de condutividade térmica de um gás paramagnético submetido a um campo magnético constante apresentavam pequenos decréscimos; dois anos após, verificou-se que o mesmo ocorría com o coeficiente de viscosidade cisalhante do gás; estes fenômenos foram denominados de efeito Senflieben.

$O$ estudo do efeito Senftleben só foi retomado no inicio década de 1960 por Beenakker e colaboradores [16], os quais observaram que o fenômeno ocorre tanto para gases paramagnéticos quarto para diamagnéticos (efeito Senftleben-Beenakker).

Do ponto de vista teórico o tratamento do problema segundo a teoria cinética de gases é mais complexo do que o empregado para gases monoatônicos, por duas razôes: primeira, que as moleculas de gasez poliatómicos apresentam graus de liberdade internos (rotação. vibraçäo e excilaçăo eletrờnica); segunda, que sob campo magnético aplicado o gás poliatômico torna.se um melo anisotropico de modo que os coefientes de transporte adquirem um carater tensorial.

Com o objetivo de determinar os coeficientes de transportepara ofeito Senftleben-Beenakker desenvolvenos uma solução para a equaçäo de Bolbzmann através do método combinado de Chapman-Enskog e Grad. Para isso, consideramos as moleculas constituintes do gás como esteras rigidas, perfeitamente elásticas e perfeitamente rugosas. As esferas apresentam energia interna de rotaçäo que, numa colisảo binária, pode ser convertida em energia de translaçấo e vice-versa. Una caracteristica deste motelo, denominado modelo de Bryan [17], é que a velocidade relativa dos pontos das duas esferas, que entram em contato na colisão, é revertida.

O emprego do modelo das esferas rugosas condua a uma maior facilidade nos cáloulos algébricos. desde que näo necessitamos de uma grande número de coordenadas para orientar a molecula no espaço, como ocorre com outros modelos moleculares existentes (esferocilindros, por exemplo). No entanto, a reversäo do sentido da velocidade relativa nas colisòes conduz a salores altos para os coeficientes macroscópicos oblidos pois, mesmo moléculas que se to. azm levemente ten suas velocidades relativa revertidas. Desta forma, nossa expectativa näo A de obter valores acurados para os coefcientes te transpote mas sim. uma determinaçă 
sistemática dos coeficientes de transporte através do uso do método combinado de Chapman. Enskog e Grad para gases poliatốmicos.

Para moléculas com graus de liberdade internos de zotaçäo Kagan e Afanase'v [20] mostra" ram que a funço de distribuição para o gás além de depender do vetor velocidade transiacional é também funçâ do psendovetor velocidade angular da molécula. Nesta nova formulaçäo grandezas que dependem da velocidade angular, denominadas polatizaçöes, podem influenciar nos valores dos coeficientes de transporte do gás. Desta forma, analisamos a influencia de duas polarizaçöes $\left(\varepsilon_{i j k} C_{j} \Omega_{k}\right.$ e $\left.\Omega_{\langle i} \Omega_{j>} C_{k}\right)$ que podem contribuir para os coeficientes de condutividade térmica e uma polarizacäo $\left(\Omega_{<i} \Omega_{j\rangle}\right)$ que pode contribuir para as coeficientes de viscosidade cisalhante.

Para obter os coeficientes de condutividade térmica é necessário exprimir grandezas tensom riais em termos do pseudovetor campo magnético e do vetor gradiente de temperatura. Isto é feito com bastante clareza através de teoremas de representação [21] os quais nos fornecem uma maneira sistemática para o cálculo de novas aproximaçöes para os coeficientes de condutítidade térmica se introduzimos novas polarizaçồ na teoria. Estas representaçōes tensoriais bem como as inversoes tensoriais empregadas no cálculo dos coeficientes de viscosidade cisalhante săc apresentadas no apêndice $\mathbf{B}$.

O cálculo algébrico das integrais de colisão, isto é, do segundo membro da equaçào de Boltzmann, são longos e trabalhosos. Em sua maioria eles foram realizados utilizandore os programas computacionaís Mathematica e Maple. No apêndice $G$ apresentamos um cálculo detalhado para uma destas integrais tipicas.

\section{Equações Linearizadas de Burnett}

No capítulo 5 obtemos as equacôes linearizadas de Burnett para gases poliatómicos de duas formas distintas: através da teoria cinética de gases, com base na equação de Boltzmann, segundo uma teoria fenomenológica basejda na teoria termodinâmica estendida. Uma explanaçăo dos metodos empregados ê dada a seguir.

O escoamento de gases rarefeitos é tradicionalmente descrito através de equaços que ex. primem leis de conservaçä́ (massa. momento linear e energia) complementadas por equaçoes constitutivas para o tensor pressäo (lei de Navier-Stokes) e para o vetor fluxo de calor (lei de Fourier). Em teoria cinética elas correspondem às soluçôes da equaçào de Boltzmann obtidas com a segunda aproximaçäo para a função de distribuiçäo utilizada no método de Chapman. Enskog. sendo que a primeira aproximaçăo corresponde ao estado de equilibrio onde o tensor pressão é somente una pressão e ofluxo de calor nulo. As equagóes de Navier-5tokes e Fourier envolvem derivadas espaciais de primeira ordem da velocidade hidrodinamica e da temperă ura do gas. respectivamente. 


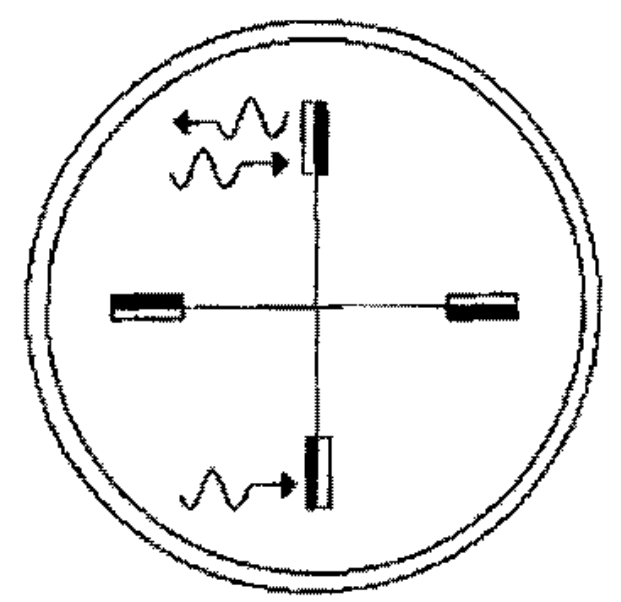

Figura 1: Radiometro de Crookes.

A primeira equação constitutiva para o tensor pressâo com termos alërn daqueles que compóen a equação de Navier-Stokes foi obtida por Maxwell [22], ao procurar explicar a fôrça de radiometro. Maxwell obteve que o deviante do tensor pressão é proporcional ao deviante da derivada espacial de segunda ordern da temperatura.

Para gases monoatómicos rarefeitos, a equação constitutiva completa para o tensor pressão contendo não somente derivadas de segunda ordem dos campos básicos (densidade, velocidade e temperatura) mas também quadrados e produtos de derivadas de primeira ordem, foi realizada por Burnett [10]; a equaçăo constitutiva para o vetor Aluxo de calor foi obtida por Chapman e Cowling [9]. Estas equaçōes são conhecidas como equaçôes de Burnett.

As equaçôes linearizadas de Burnett, obtidas no capitulo 5, desempenham papel importante na explicação de vărios fenômenos que tratam de gases poliatônicos rarefeitos. Como ilustração. destacamos dois deles: a fốrça de radiômetro e o efeito Scott.

O fenômeno do radiồmetro, observado por Crookes em 1873, ocupou a atenção dos físicos na segunda metade do século passado e pode ser considerado um marco no desenvolvimento da teoria cinética dos gases [23].

O radiồmetro consiste de um invólucro de vidro com ar faréleito no seu interior e um sistema de pás capaz de rodar em torno de um eixo fixo (veja figura 1). Consideremos dois feixes de luz incidindo ortogonaimente em duas pás diametralmente opostas; a luz incidente sobre a face preta da pá é absorvida transferindo momento linear da radiaçăo para a pá; a luz incidindo sobre a face branca é refletida transferindo à pá o dobro do mornento linear incidenté; desta forma, o conjunto deveria rodar no sentido horário. No entanto, o que se observa é que o sistema gira no sentido anti-horário.

Para obter uma explicação qualitativa consideremos o papel desempenhado pelo ar rarefeito no interior do radiômetro. A face preta da pá, que absorve luza torma-se mais aquecida $(T+\Delta T)$ 


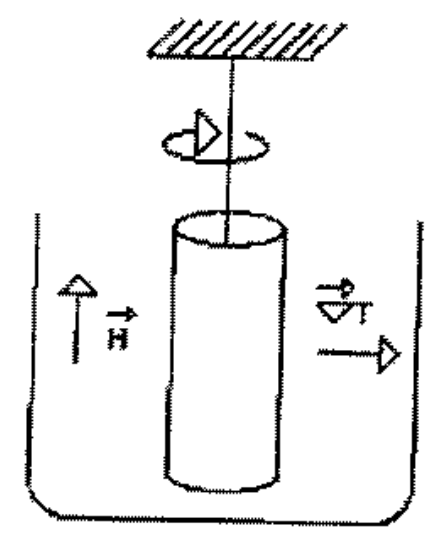

Figura 2; Arrajo experimental para o efeito Scott.

que a face branca (T) da pá que reflete luz. As moléculas do găs rarefeito que colidem com a face preta mais aquecida fetornam ao gás com urra energía cinética major que aquelas que colidem com a face branca mais fria. Assim, a transferência líquida de momento linear tas moléculas é maior para as pás com faces escurecidas resultando num movimento no sentido antîhorârio. Maxwell [22] propôs uma explicaçăo quantitativa para o fenốmeno considerando que a pressä̃o sobre as pás está relacionada com gradientes de segunda ordem da temperatura.

Em 1967, foi observado por Scoth, Stumer e Williamson [24] que un gás poliatônico rarefeito $\left(\mathrm{O}_{3}, \mathrm{~N}_{2}\right.$ ou $\mathrm{CH}_{4}$, por exemplo) limitado por dois cilindros coaxiais pode exercer um torque sobre o cilindro interno se um gradiente te temperatura é estabelecido entre os dois cilindros e um cumpo magnético é aplicado paralelamente aos éxos dos cilindros (veja figura 2). Este fenömeno s conhecido na literatura como efeito Scott. Verifica-se que a direça do torque coincide com a do campo magnético aplicado $\mathrm{H}$ e o seu sentído é revertido se um deles, o campo magnético ou gradiente de temperatura, é revertido.

A origem do torque exercido pelo gás sobre o cilindro interno pode ser considerada como de. vido a transporte de momento angular do gás para o cilindro. Levi e Beenakker [25] mostraram que o termo proporcional ao gradiente de segunda ordem da temperatura $\dot{e}$ uma das causas para o torque observado.

No capítulo 5, determinamos com a teoria cinética a terceira aproximação linearizada para a funçảo de distribuiçằ através do método de Chapman-Enskog considerando as moléculas como esferas rigitas, perfeilamente elásticas e perfeitamente rugosas (modelo de Bryan). Com a terceira aproximaça obtemos as equaçoes constitutivas para o tensor pressão e para o vetor fuxo de calor que constituem as equaçōes linearizadas de Burnetz. O tensor pressâo apresenta Les termos que näo ocorrem na equaçăo correspondente para un gás monoatónico rarefeito. Im deles é bem conhecido sendo proporcional ao divergante da velocidade do gás tendo cono 
coeficiente a viscosidade volumétrica. Os outros dois säo proporcionais ans laplacianos da temperatura $e$ da densidade e estäo associados as pressöes térmica e de densidade, respectivamente. $O$ vetor fluxo de calor contém termos proporcionaís a derivadas espaciais de segunda ordem da velocidade macroscópica do gás, denominados fluxos viscosos de calor. Todos os coeficientes destes termos săo inversamente proporcionais â pressão do gás.

Os coeficientes nestas equaçoes generalizadas de Navier-Stokes e Fourier sào completamente determinados em termos dos coeficientes oblidos com a segunda aproximação para a função de distribuição do método de Chapman-Enskog os quais, por sua vez, dependem da massa e do diämetro molecular.

As equaçôs linearizadas de Burnet säo também obtidas através de uma teoria feromenológica baseada na termodinâmica estendida para um gás poliatômico com uma variável interna, desenvolvida por Kremer $[26,27]$. O estado macroscópico do gás é descrito pelos dezessete campos escalares seguintes: densidade de massa, densidade de momento linear, tensor pressào, fluxo de calor translacional, densidade de energia da variável interna e fuxo de energia da variâvel interna. Horm igual número de equaçöes de balanço para estes campos básicos.

Com as equaçốtes de balanço para os campos bắsicos utilizamos o método iterativo de Maxwell [28] da teoria cinética para determinar as equacöos linearizadas de Burnett para o tensor pressão e vetor fuxo de calor. Estas equaçóes contem coeficientes de transporte que podem ser determinados experimentalmente ou calculados através da teoria cinética.

Na teoria fenomenológica a temperatura é associada com o traço do tensor pressăo enquanto que na teoria cinética ela é associada com a energia especifica interna total. Com a finalidade de comparar as duas teorias associamos as temperaturas definidas nestas teorias de modo que os coeficientes de transporte da teoria fenomenológica podem ser identificados em termos de seus correspondentes da teoria cinética.

$O$ conceito de termperatura desempen ha um papel fundamental em termotinămica principal. mente porque ela é considerada uma grandeza facilmente mensurável. Utilizando a condiçäo de salto para a temperatura numa parede termométrica verificamos que, para processos próximos do equilibrio onde termos năo lineares tos campos podem ser negligenciados, as temperaturas definidas pelas duas teorias săo continuas; entretanto, para processos em que os termos näo lineares säo relevantes as temperaturas são descontínuas. Nestes processos, o conceito de temperatura perde muito do seu valor como variảvel termodinâmica. 


\section{Capítulo 1}

\section{Fundamentos da Teoria Cinética de Gases Poliatômicos}

\subsection{Introdução}

O objetivo deste capítulo é descrever os fundamentos da teoria cinética de gases poliatomicos e seu desenvolvimento tem como base as referencias [9] e [29] a [32].

A teoria cinébica considera gases como sendo constituidos de um nimero muito grande de moléculas que, na maior parte do tempo, movimentam independentemente através do volume do gás. O movimento livre e brevemente interrompido quando uma molecula colide com as paredes do recipiente que a contém ou com outras moléculas.

Se a energia potencial de interação entre as moléculas puder ser desprezada em comparaçào com suas energias cináticas temos um gás ideal e, em caso contrário, um gás denso ou real.

Para se ter uma estimativa de comprimentos característicos, considererrios as moléculas do gás hélo como esferas rigidas com diametro $a=2,18 \times 10^{-10} \mathrm{~m}$. Como um mol de gats, à temperatura de $273,15 \mathrm{~K}$ e pressão de uma atmosfera $\left(1,01 \times 10^{5} \mathrm{~Pa}\right)$, ocupa um volume de $2,24 \times 10^{-2} \mathrm{~m}^{3}$ e tem um número de moléculas igual ao número de $\mathrm{A}$ vogadro $N_{A}=6,02 \times 10^{23}$ moléculas/mol, a densidade de número de moléculas é da ordem de $2,69 \times 10^{25}$ moléculas $/ \mathrm{m}^{2}$.

Se imaginarmos o volume molar dividido em células cúbicas, com uma molécula por célula, o volume de cada cêlula será de $3,72 \times 10^{-26} \mathrm{~m}^{3}$ aproximadamente, que resulta num comprimento de aresta de $3,34 \times 10^{-9} \mathrm{~m}$. Portanto, podemos concluir que a distância média $d$ entre as moléculas $\hat{A}$ da ordem do comprimento de uma aresta, isto é, cerca de quinze vezes o dianmetro molecular.

Se considerarmos uma molécula teste que se move com uma velocidade a densidade de número de partículas é $n$, podemos dizer que, por unílate de tempo, a molḱtula teste colide com todas as outras moléculas cujos centros estäo no cilindro com área de base za $a^{2}$ e altura $y$. O número de colisóes por unidade de tempo, denominato de freqüuencia de colisäo, é $y=n \pi a^{2} v$. 
O tempo médio entre colisöes é o inverso da trequência de colisōes, ou seja

$$
\tau=\frac{1}{\nu}=\frac{1}{n \sin ^{2} t^{2}}
$$

e o livre camunho médio, que corresponde à distãncia média percorrida por uma molécula entre duas colisoes, dado atravis de

$$
l=v r=\frac{1}{n \pi a^{2}}
$$

Por outro lado, o tempo de colisäo $\tau_{c}$, que representa o tempo de interaço entre duas moléculas, e considerado como sendo diretamente propocional ao alcance efetivo das forças intermoleculares e inversamente proporcional à velocidade da molécula. $\mathrm{O}$ alcance efetivo das forças por sua vez, da ordem do diâmetro molecular. Conseqüuntemente, temos que

$$
\tau_{c} \approx \frac{a}{y}
$$

Assim, com uma densidade do número de moléculas $n=2,69 \times 10^{25} \mathrm{moléculas} / \mathrm{m}^{3}$ para moléculas com diâmetro $a=2,18 \times 10^{-10} \mathrm{~m}$ e velocidade térmica média $=3,0 \times 10^{2} \mathrm{~m} / \mathrm{s}$, temos:

- livre caminho médio: $l=2,5 \times 10^{-7} \mathrm{~m}$,

- tempo médio entre colisões: $\tau=8,3 \times 10^{-10} \mathrm{~s}$,

- tempo de colisão: $\tau_{c} \approx 10^{-1 / 2} s$.

Com estes valores concluimos que, para um gás ideal, o livre caminho médio l é carca de oitenta vezes a distancia média d entre moléculas e que o tempo médio $\tau$ entre as colisóes é cerca de mil vezes o tempo de colitiono $\tau_{c}$.

En geral, gases constituitos de moléculas neutras a pressões até a ordem de 100 atm podem ser considerados como gases ideais pois, neste intervalo, a ocorrênáa de colisùes ternárias

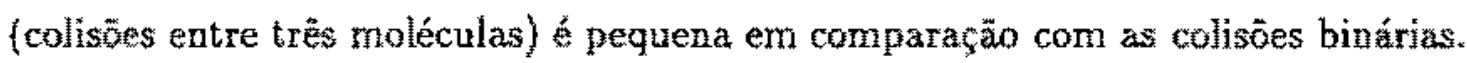

Por outro lado, gases com temperaturas antre $50 \mathrm{~K}$ e $100000 \mathrm{~K}$ podem ser tratados cono gases clássicos ideais pois, neste intervalo, nem os efeitos quânticos nem os relativinticos sä importantes.

Esta tiltima observação nos permite descrever o movimento das moléculas a partir das leis da mecânica clássica Newtoniana. No entantô, estes limites nào säo absolutos pois a densidade do número de moléculas não pode ser considerada como arbitrária. Para assegurar que nestes limites os efeitos quânticos e relativisticos podem ser desprezados, as seguintes condiçôs devem ser satisfeitas: 
(i) o comprimento de onda de de Broglie $\lambda_{B}$ associado a uma molecula, deve ser muito menor que a distância média $d$ entre as moléculas:

$$
\lambda_{B}=\frac{h}{(3 m k T)^{1 / 2}} \ll d,
$$

onde $h=6,626 \times 10^{-34} \mathrm{~J}$.s é a constante de Planck;

(ii) a velocidade térnica média de uma molécula deve ser muito menor que a velocidade da luz no vacuo $t=2,998 \times 10^{8} \mathrm{~m} / \mathrm{s}$.

\subsection{Gases Poliatômicos}

Os gases poliatômicos podem ser considerados cono sendo constituídos por moléculas con graus de liberdade internos (ou modos de movimento) com os quais podemos associar estados de energia interna. Estes estados de energia interna podem ser: a energia rotacional da molécula, a energia de vibração dos átomos que compõem a molếcula ou a energía de excitação eletrônica das moléculas.

Como os átomos, moléculas apresentam estados eletrổnicos que säo dífíceis de serem exci tados já que requerem vários elétronsmvolt de energia acima do estado fundamental (1 elétronvolt de energia corresponde a uma temperatura aproximada de $11600 \mathrm{~K}$ ). Para temperaturas moderadas os estados eletrónicos excitados das moléculas säo inacessiveis, de modo que seräo ignorados para os nossos objetivos.

No desenvolvimento deste trabalho consideraremos as moléculas constituintes do gás po" liatônico como esferas rígidas e rugosas com diâmetro a, tendo a massa $m$ distribuída simetricamente em torno do seu centro geométrico. Assim, a molécula apresenta trés graus de liberdade translacionais associados à energia de translaçào $\frac{1}{2} m c^{2}$ do seu centro de massa e très graus de liberdade rotacionais associados anergiz de rotaça $\frac{1}{2} l \omega^{2} ; c$ é a velocidade do centro de massa, w a velocidade angular em torno de um eixo que passa pelo centro de maska e I é o monento de inércia da molécula em relaçấo a esse eixo.

Para um gás ideal a relaço entre os calores especificos a pressão constante co a volume constante $c_{t}$, é dada por $[3 x\}$

$$
\gamma=\frac{c}{c_{y}}=1+\frac{2}{f},
$$

onde $f$ é o número de graus de liberdade da molécula. Para o nosso modelo molecular esférico $f=6$, de modo que $\gamma=\frac{4}{3} \simeq 1,33$. Na tabela 1.1, obtida de [34], apresentamos valores ca* racteristicos para alguns gases poliatömicos que preenchem os requíitos deste modelo. Nesta labela $\Theta_{r o t}=\frac{h^{2}}{2 l k}$ a temperatura característica de rotação e $\Theta_{v i b}=\hbar \nu_{o s e}$ a temperatura 


\begin{tabular}{|c|c|c|c|r|}
\hline Gas & $\gamma=c_{p} / c_{v}$ & $\begin{array}{c}\Delta T \\
(\mathrm{~K})\end{array}$ & $\begin{array}{c}\Theta_{\text {rot }} \\
(\mathrm{K})\end{array}$ & $\begin{array}{r}\Theta_{\text {wit }} \\
(\mathrm{K})\end{array}$ \\
\hline $\mathrm{CH}_{4}$ & 1,33 & $60<T<200$ & 7,4 & 1876 \\
$\mathrm{CF}_{4}$ & 1,33 & $60<T<80$ & 0,4 & 628 \\
$\mathrm{CD}_{4}$ & 1,33 & $80<T<160$ & 3,9 & 1546 \\
\hline
\end{tabular}

Tabela 1.1: Temperaturas características para alguns gases poliatômicos.

\begin{tabular}{|c|c|c|c|c|c|}
\hline Gás & $\begin{array}{c}m \\
10^{-26} \mathrm{~kg}\end{array}$ & $\begin{array}{c}a \\
10^{-10} \mathrm{~m}\end{array}$ & $\begin{array}{c}1 \\
10^{-47} \mathrm{~kg} \cdot \mathrm{m}^{2}\end{array}$ & \multirow{1}{*}{$\begin{array}{c}\eta \\
10^{-6} \mathrm{Pas}\end{array}$} \\
\hline $\mathrm{CH}_{4}$ & 2,66 & 4,12 & 5,47 & 0,048 & 10,33 \\
$\mathrm{CF}_{4}$ & 14,61 & 5,04 & 101,30 & 0,109 & 16,17 \\
$\mathrm{CD}_{4}$ & 3,33 & 4,08 & 10,44 & 0,075 & 11,80 \\
\hline
\end{tabular}

Tabela 1.2: Valores característicos para alguns gases poliatômicos a temperatura de $273 \mathrm{Ke}$ pressāo de $1,01 \times 10^{5} \mathrm{~Pa}$.

característica de vibraçăo, onde $\hbar=\frac{h}{3 \pi}$ e $k$ säo, respectivamente, as constantes de Planck e Boltzmann: vasc a frequência característica de vibração da molécula. 0 valor $\gamma=\frac{1}{3} e$ verificado, aproximadamente e com os dados experimentais disponíveis, nos intervalos de temperatura apresentados na coluna para $\Delta T$. Nestes intervalos, o metano $\left(\mathrm{CH}_{4}\right)$, o tetrafluoreto de carbono (CF $)$ e o metanom $\left(\mathrm{CD}_{4}\right)$ näo apresentam estados vibracionais excitados.

$\mathrm{Na}$ tabela 1.2 os valores para os diametros moleculares a para $\mathrm{CH}_{4}$ \& $\mathrm{CH}_{4}$ foram calculados a partir de valores experimentais dos coeficiente de viscosidade cisalhante $n$ [35], atraves de [veja equaç̄ôn $(3.62)]$

$$
\eta=\frac{15}{8 a^{2}}\left(\frac{m k T}{\pi}\right)^{1 / 2} \frac{(\kappa+1)^{2}}{(13 \kappa+6)}
$$

onde o momento de inércia adimensional é dado por

$$
N=\frac{4 I}{m a^{2}}
$$

O coeficiente de viscosidade cisalhante para $\mathrm{OCD}_{4}$ foi calculado a partit do coeficiente de viscosidade do $\mathrm{CH}_{4}$, através da relaçâto (36)

$$
\mu_{C D_{1}}=1,142 \mu_{\mathrm{CH}_{*}} .
$$

O falo de gases poliatomicos apresentarem energias moleculares internas implica em difem renças significativas con os gases monoatómicos. Estas diferenças estäo relacionadas com o 


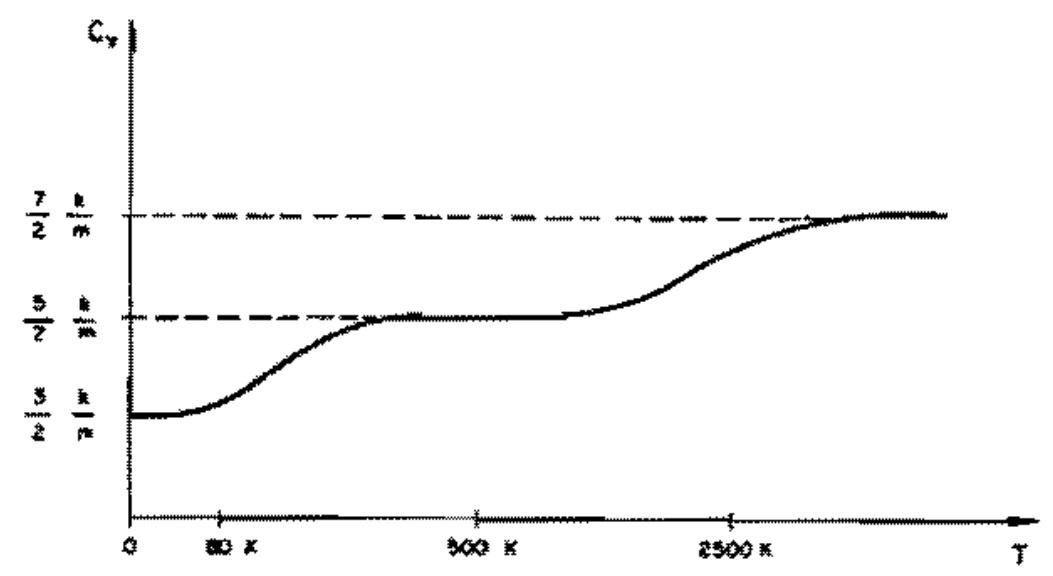

Figura 1.1: Valores experimentais para o calor especifico ck para o gás $\mathrm{H}_{\text {. }}$

calor especifico a volume constante $c_{y}$ ecm a relaçäo $\mathrm{N} / \mathrm{\eta}$ entre o coeficiente de condutividade tểmica $\lambda$ e coeficiente de viscosidade de cisalhamento $\eta$. Para um gás monoatômico $c_{v}$ é práticamente constante e dado por $c_{\mathrm{t}}=\frac{3}{2} \frac{k}{\mathrm{~m}}$, sendo $m$ a massa da partícula do gás. Para um gás poliatômico $c_{v}$ é uma funçäo da temperatura. A figura 1.1 mostra o comportamento de $c_{v}$ en funçăo da temperatura para um gás de hidrogênio. Para temperaturas muito baixas, $c_{v}$ coincide com o valor do calor específico a volume constante de um gás monoatômico (cy $=\frac{3}{2} \frac{k}{m}$ ). Este fato pode ser interpretado considerando que, a temperaturas muito baixas, as energias de rotaça e vibraçăo nāo estäo ainda excitadas. A medida que a temperatura cresce a energia rotacional é excitada e o calor especifico a volume constante cresce até $c_{v}=\frac{5}{2} \frac{5}{2}$. continuo aumento da temperatura faz com que senergia de vibraçào da molécula seja excitada de modo que o calor especifico cresce até o valor $c_{v}=\frac{7}{2} \frac{k}{m}$.

Os graut de liberdade referentes à energia de excitaçăo eletrônica sơ săo ativados a tempe raturas mais altas (em particular, para pressótes moderadas molécula $\mathrm{H}_{2}$ se dissocia antes de atingir o valor $c_{z}=\frac{\tau}{2} \frac{k}{m k}$ ).

A relação $\lambda / 7$ para gases monoatomicos é praticanente uma constante e dada por [9]

$$
\frac{\lambda}{\eta}=\frac{15}{4} \frac{k}{m}=\frac{5}{2} c^{z}
$$

onde $c_{t}^{T}=\left(\frac{\partial^{T}}{\partial T}\right)$ é o calor especifico a volume constante correspondente de translaçào $\varepsilon^{T}=\frac{3}{3} \mathrm{k} T$.

Várias fórmulas foram propostas para a relaçăo A/n para gases poliatônicos (veja. por exemplo. [37]). A mais antiga delas é devida a Eucken [38], que dividiu a condutividade 


\begin{tabular}{|l|l|l|}
\hline Gás & {$\left[f_{E}\right]_{\exp }$} & {$\left[f_{E}\right.$ teo } \\
\hline $\mathrm{He}$ & 2,45 & 2,50 \\
\hline $\mathrm{Ne}$ & 2,52 & 2,50 \\
\hline $\mathrm{Ar}$ & 2,48 & 2,50 \\
\hline $\mathrm{H}_{2}$ & 2,02 & 1,90 \\
\hline $\mathrm{CH}_{4}$ & 1,77 & 1,75 \\
\hline
\end{tabular}

Tabela 1.3: Valores experimentais e teóricos para o número de Eucken.

térmica em duas partes:

$$
\lambda=\eta\left(f_{T} c_{w}^{T}+f_{r} c_{w}^{t}\right)
$$

O primeiro termo do lado direito está associado ao transporte de energia translacional pela molécula enquanto que o segundo considera o transporte de energia da variáutel interna: $c_{w}^{I}=$ $\left(\frac{\partial e^{i}}{\partial T}\right)_{t}$ é calor especifico a volume constante correspondente à energia especifica da variável internat $\varepsilon^{l}$.

Eucken escolheli os valores $f_{T}=\frac{5}{2}$ ef $f=1$, de modo que podemos escrever para a equaçă (1.10):

$$
\frac{\lambda}{\eta}=\frac{5}{2} c_{v}^{T}+c_{v}
$$

A equaçăo anterior pode também ser escrita como

$$
f_{E}=\frac{\lambda}{\eta c_{y}}=\frac{9}{2 f}+1
$$

onde $f$ to número de graus de liberadade da molécula $e$

$$
c_{w}=c_{y}^{*}+c_{y}^{t}=\frac{f}{2} \frac{k}{m}
$$

é o calor especifico total a volume constante. A combinação adimensional $f_{E}=\frac{1}{n} \frac{1}{e}$ denominada de número de Eucken.

As partículas de gases monoatômicos apresentam três graus de liberdade translacionais

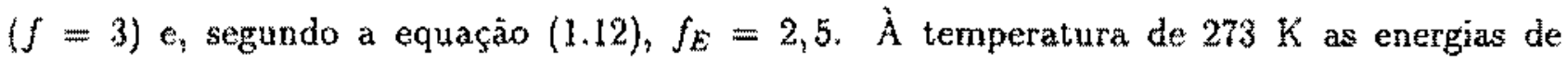
rotaçăo das moléculas do gases hidrogênio $\left(\mathrm{H}_{2}\right)$ e metano $\left(\mathrm{CH}_{4}\right)$ estâo completamente excitadas enquanto que as energias de vibração ainda näo estäo. Podemos afirmar entảo, que o número de सraus de liberdade para a molécula de hidrogênio é $f=5$ pois é uma molécula linear, enquanto que para molécula esférica do metano temos $f=6$.

A tabela 1.2 apresenta, para alguns gases monoatomicos e poliatomicos, os valores experimentais (obtidos de [9]) e leóricos (obtidos da equaçăo (1.12)] para o número de Eucken, para a temperalura de $273 \mathrm{~K}$. Verificamos uma boa concordância entre valores teóricos e os obtidos experimentalmente. 
Como a fórmula de Eucken (1.12) independe ta temperatura, decorre que os valores teóncos são válidos para quaisquer temperaturas; obviamente, ela não deve ter validade geral.

\subsection{Gases Poliatômicos de Moléculas Rugosas}

Diversos modelos moleculares foram introduzidos para descrever as colisoes inelásticas entre as moléculas de gases poliatômicos, dentre os os quais citamos:

(i) o modelo de esferas lisas, elásticas e rugosas em que duas esferas se aderem sem deslizar numa colisào binária; este modelo foi introduzido por Bryan [17] e utilizado por Pidduck [39] no cálculo de coeficientes dè transporte;

(ii) o modelo de esferas lisas em que o centro de massa e o centro gemétrico da tatera năo coincidem (loaded spheres), introduzido por Jeans [40];

(iii) o modelo de esferocilindros de Kihata [41], que consiste de cilindros com semi-esteras em suas extremidades.

Dentre estes modelos, o das esferas elasticas e rugosas e 0 mais simples de ser tratado matematicamente, desde que o estado dinamico da molécula é caracterizado por somente três variaveis: a posiçăo a velocidade linear do seu centro de massa além da velocidade angular em torno de um eixo que passa pelo centro de massa da molécula năo sẫo necessárias variáveis adicionais para especificar a orientaça da molécula no espaço. É o modelo que empregaremos no desenvolvimento deste trabalho.

Na descriçâo de gases poliztómicos consideraremos que as moléculas que constituem o gás possuem anergias cinética de translaçăo e interna de rotação. Um modelo clássico para tais moléculas toi introduzido por Bryan [17] que considerou as como esferas rigidas, perfeitamente elásticas e rugosas, com a massa distribuida simetricamente em torno do centro da esfera.

Segundo aste modelo, numa colisào elástica binária os pontos das esferas que entram em contato se aderem sem deslizamento inicialmente, cada esfera é deformada pela outra e. em seguida, a energia de deformação é reconvertida em energias cinética de translação e rotăçăo, nenhuma energia sendo perdida. Como consequencia, a velocidade relativa dos pontos das esferas que entram em contato é revertida após a colisão.

Este modelo é relativamente simples desde que a caracterização de uma molécula é feita através de apenas dois parämetros: o diâmetro a e o momento de inércia $I$. Não são necessários parâmetros adicionais como. por exemplo, indicar a orientaçảo da molécula no espaço.

O modelo molecular de esferas rugosas foi empregado pela primeira vez por Pidduck [39] no calculo para os coeficientes de transporte de gases poliatômicos. 


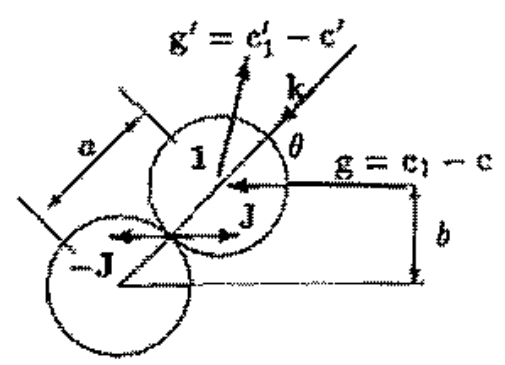

Figura 1.2: Colisâo elástica entre duas esferas rugosas.

\subsubsection{Dinâmica da Colisão Binária}

Vamos analisar a colisão elástica entre duas moléculas representadas por esferas rígidas, perfeitamente elásticas e rugosas, com diametro a, massa m e momento de inércia $f$. Na colisào ocome uma troca de energias cinéticas de translaçẫo e rotaçăo através da aça de impulsos mítuos entre as moléculas. Denotaremos por $\left(c_{1}, \omega\right)$ e $\left(c_{1}, w_{1}\right)$ as velocidades linear angular das duas moléculas antes da colisão por $\left(c^{*}, w^{\prime}\right)$ e $\left(\mathrm{c}_{\mathrm{t}}^{7}, \omega_{1}^{\prime}\right)$ suas correspondentes velocidades após a colisảo. Nosso objetivo nesta seção é determinar as ralaçoes que permitem obter as velocidades moleculares pós-colisionais $\left(c^{t}, \omega^{\prime} ; c_{1}^{\prime}, \omega_{1}^{*}\right)$ em termos dus velocidades pré-colisionais

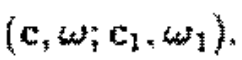

Definimos a velocidade linear relativa dos centros de massa das esferas, antes da colisăo, por

$$
\mathrm{g}=\mathrm{c}_{1}-\mathrm{c}
$$

e, após a colisäio, por

$$
\mathrm{g}^{\prime}=\mathrm{c}_{1}^{\prime}-\mathbf{c}^{\prime}
$$

Numa colisato binária, a molecula denotada por 1 exerce um impulso - I sobre a molécula sem rotulo $e$, simultaneamente, experimenta um impulso $\mathrm{J}$ o que provoca a alteraçäo de suas velocidader pré-colisionais (veja figura 1.2).

$O$ vetor de colisăo (ou vetor apsidal) é definido por

$$
k=\frac{g-g^{\prime}}{\left|g-g^{\prime}\right|}
$$

tem a direçâ da retáa que une os centros da moléculas no instante da colísão e é dirigido da molécula 1 para a molécula sem rótuló alẻm dis5o, $\mathrm{k}$ bissecta o ángulo formado pelas direçöes das velocidades relativas $\mathrm{g}$ e $\mathrm{g}^{*}$. Na figura $1,2, x=\pi-20$ é o angulo de espalhamento e be parâmetro de impacto para a colisăo. 
A partir das leis de conservaçào dos momentos linear e angular para as esferas, podemos escrever as equagòes de impacto tomo

$$
\begin{array}{cl}
m \mathbf{c}^{\prime}=m \mathrm{c}-\mathrm{J}, \quad m \mathrm{c}_{1}^{\prime}=m \mathrm{c}_{1}+\mathrm{J} \\
I \omega^{\prime}=I \omega^{\prime}+\frac{a}{2} \mathrm{k} \times \mathrm{J}, \quad I \omega_{1}^{\prime}=I \omega_{1}+\frac{a}{2} \mathrm{k} \times J .
\end{array}
$$

Par outro lado, se designarmos por $V$ a velocidade relativa dos pontos das esferas que entram em contato antes do choque, temos

$$
V=c_{1}-\frac{a}{2} k \times w_{1}-c-\frac{a}{2} k \times w
$$

após o choq̣ue

$$
\mathrm{V}^{\prime}=c_{1}^{t}-\frac{a}{2} \mathrm{k} \times \omega_{1}^{\prime}-\mathrm{c}_{1}^{z}-\frac{a}{2} \mathrm{k} \times w^{t}
$$

Para esferas rugosas, a velocidade relativa dos pontos que entram em contato é revertida após a coljsão ou seja,

$$
V^{\prime}=-\mathbf{V}
$$

e subtraindo a equação $(1.20)$ da (1.19), tendo en conta a equaçäo (1.21), resulta

$$
2 \mathbf{V}=c_{1}-\frac{a}{2} \mathbf{k} \times \omega_{1}-\mathbf{c}-\frac{a}{2} \mathbf{k} \times \omega-\mathbf{c}_{1}^{\prime}+\frac{a}{2} \mathbf{k} \times \omega_{1}^{\prime}+\mathrm{c}_{1}^{\prime}+\frac{a}{2} \mathbf{k} \times \omega^{\prime}
$$

Com o emprego das equaçöes $(1.17)$ e $(1.18)$, obtemos

$$
\mathbf{V}=-\frac{1}{m} \mathbf{J}+\frac{a^{2}}{k_{l}}[(\mathbf{J} \cdot \mathbf{k}) \mathbf{k}-\mathbf{J}]
$$

Definimos a momento de inếrcia adimensional por

$$
n=\frac{4}{m a^{2}}
$$

sendo que $k$ varia desde o valor 0 , que corresponde à concentraçăo de massa no centro da esfera, até um valor máximo de $2 / 3$ que corresponde à distribuiçào uniforme de massa na superfícte da esfera. $\mathrm{O}$ valor $*=2 / 5$ correxponde distribuiça uniforme de massa na esfera.

Assim, da equação $(1.23)$, temos

$$
\mathbf{V}=-\frac{1}{m} \mathbf{J}+\frac{1}{m k}[(\mathbf{k} \cdot \mathbf{J}) \mathbf{k}-\mathbf{J}]
$$

* multiplicando escalarmente a equaça anterior por $\mathrm{k}$, obtemos 


$$
\mathbf{V} \cdot \mathbf{k}=-\frac{1}{m} \mathbf{J} \cdot \mathbf{k}
$$

Substituindo esta equaça na $(1,25)$ e resolvendo para J. resulta:

$$
\mathfrak{J}=-\frac{m k}{(\kappa+1)}\left[\mathrm{V}+\frac{1}{\kappa}(\mathrm{V} \cdot \mathrm{k}) \mathrm{k}\right]
$$

Se substituirmos o impulso I nas equacoes de impacto (1.17) e (1.18) obtemos as velocidades finats em termos das velocidades iniciais, ou seja

$$
\begin{aligned}
& c^{\prime}=\mathrm{c}+\frac{\kappa}{(\kappa+1)}\left[\mathrm{g}-\frac{a}{2} \mathrm{k} \times\left(\omega+\omega_{1}\right)+\frac{1}{\kappa}(\mathrm{g} \cdot \mathrm{k}) \mathrm{k}\right] \\
& \mathrm{c}_{1}^{\prime}=\mathrm{c}_{1}-\frac{\kappa}{(k+1)}\left[\mathrm{g}-\frac{a}{2} \mathrm{k} \times\left(\omega+\omega_{1}\right)+\frac{1}{k}(\mathrm{~g} \cdot \mathrm{k}) \mathrm{k}\right] \\
& \omega^{\prime}=\omega-\frac{2}{a(\kappa+1)}\left\{\mathrm{k} \times \mathrm{g}-\frac{a}{2} \mathrm{k} \times\left[\mathrm{k} \times\left(\omega+\omega_{1}\right)\right]\right\} \\
& \omega_{1}^{\prime}=\omega_{1}-\frac{2}{a(k+1)}\left\{\mathrm{k} \times \mathrm{g}-\frac{\mathrm{a}}{2} \mathrm{k} \times\left[\mathrm{k} \times\left(\omega+\omega_{1}\right)\right]\right\}
\end{aligned}
$$

Notemos que, para $\mathrm{s}$ tendendo a zero, as equaçōes $(1.28)$ e $(1.29)$ se transformam em

$$
\begin{aligned}
& c^{\prime}=\mathrm{c}+(\mathrm{g} \cdot \mathrm{k}) \mathrm{k}, \\
& \mathrm{c}_{1}^{\prime}=\mathrm{c}_{1}-(\mathrm{g} \cdot \mathrm{k}) \mathrm{k}
\end{aligned}
$$

que săo as relaçōes entre as velocidades pós-colisionats e pré-colisionatis obtidas no modelo de esfera lisas, empregadas no estudo de gases monoatónicos [0]. Neste modelo, as velocidades lineares pós-colistonais independem das yelocidades angulares. Por outro lado, pelo princtio da equipartiçào da energia, as energias cinéticas médias para uma molécula estäo telacionadas por

$$
\frac{1}{2} m \overline{c_{i}^{2}}=\frac{1}{2} f \bar{\omega}=\frac{1}{8} m a^{2} \kappa \overline{\omega^{2}}=\frac{1}{2} k T
$$

ou sejk.

$$
\overline{c_{i}^{2}}=\frac{1}{4} a^{2} \bar{w}_{i}^{2}
$$

de morlo que, para $k$ iendendo a zero, o módulo de c deve ser muito menor que o módulo de aw: assim. as equacoes $(1.30)$ e (1.31) se reduzem a 

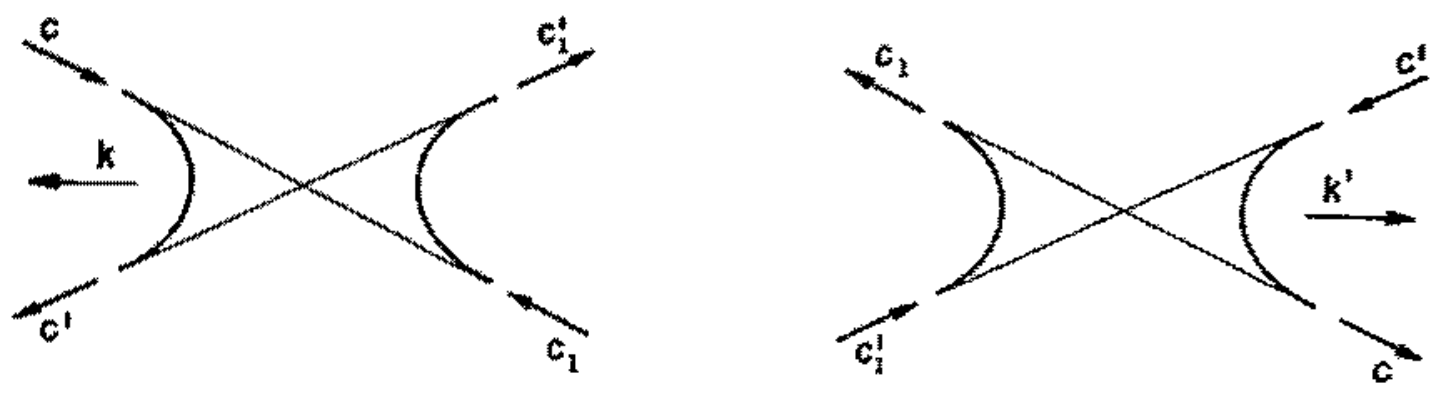

Figura 1.3: Colisöes direta e inversa de moléculas no modelo de esferas lisas.

$$
\begin{aligned}
\omega^{\prime} & =\omega+\mathrm{k} \times\left[\mathrm{k} \times\left(\omega+\omega_{1}\right)\right], \\
\omega_{1}^{\prime} & =\omega_{1}+\mathrm{k} \times\left[\mathrm{k} \times\left(\omega+\omega_{1}\right)\right],
\end{aligned}
$$

ou seja, as velocidades angulares finais independem das velocidades lineares.

Das equaçöes (1.28) e (1.29), podemos verificar que

$$
\mathrm{g} \cdot \mathrm{k}=-\mathrm{g}^{\prime} \cdot \mathrm{k}=\mathrm{g}^{\prime} \cdot \mathrm{k}^{\prime}
$$

No modelo de esferas lisas, a cada choque direto entre duas moléculas com velocidades $\mathrm{c}$ e $\mathrm{c}_{1}$, vetor de colisäo $\mathrm{k}$ e que resultam em moléculas com velocidades pós-colisionais $\mathrm{c}^{\prime}$ e $\mathrm{c}_{1}^{\prime}$, corresponde um choque inverso entre moléculas com velocidades pré-colisionais $\mathrm{c}^{\prime}$ e $\mathrm{c}_{1}^{t}$, vetor de colisảo $\mathrm{k}^{\prime}=-\mathrm{k}$ e que resultam em moléculas com velocidades pós-colisionais $\mathrm{c}$ e $\mathrm{c}_{1}$. Isto pode ser verificado através das equaçōes (1.32), (1.33) e (1.38), e está ilustrado na figura 1.3.

Para o caso de gases poliatômicos no modelo de moléculas esféricas rugosas näo existe um choque inverso, ou seja. moléculas com velocidades pré-colisionais $\left(c^{\prime}, \omega^{\prime} ; c_{1}^{\prime}, \omega_{1}^{\prime}\right)$ que resultam em moléculas com velocidades pós-colisionais $\left(c, \omega ; c_{1}, \omega_{1}\right)$ e vetor de colisăo $\mathrm{k}^{b}=-\mathrm{k}$, como pode ser verificado das equaçoes (1.28) e (1.31). Assim, denotaremos por $\left(\mathbf{c}^{*}, \omega^{*}\right)$ e $\left(c_{1}^{*}, \omega_{1}^{*}\right)$ as velocidades pré-colisionais que correspondem às velocidades pós-colisionais $(c, \omega)$ e $\left(c_{1}, \omega_{1}\right)$, com vetor de colisāo $\mathrm{k}^{*}=-\mathrm{k}$. Esta é denominada de colisäo de restitutçio e corresponde à colisäo direta descrita pelas equaçóes (1.28) a (1.31).

Com estas consideraçòes podemos escrever as velocidades pós-colisionais em termos das pré-colisionais, das equaçóes (1.28) a (1.31), como

$$
c=c^{*}+\frac{\kappa}{(\kappa+1)}\left[g^{*}-\frac{a}{2} k^{*} \times\left(\omega^{*}+\omega_{1}^{*}\right)+\frac{1}{\kappa}\left(g^{*} \cdot k^{*}\right) k^{*}\right]
$$




$$
\begin{aligned}
& \mathrm{c}_{1}=\mathrm{c}_{1}^{*}-\frac{n}{(k+1)}\left[\mathrm{g}^{*}-\frac{a}{2} \mathrm{k}^{*} \times\left(\omega^{*}+\omega_{1}^{*}\right)+\frac{1}{\kappa}\left(\mathrm{g}^{*} \cdot \mathrm{k}^{*}\right) \mathrm{k}^{*}\right] \\
& \omega=\omega^{*}-\frac{2}{a(k+1)}\left\{\mathrm{k}^{*} \times \mathrm{g}^{*}-\frac{a}{2} \mathrm{k}^{*} \times\left[\mathrm{k}^{*} \times\left(\omega^{*}+\omega_{1}^{*}\right)\right]\right\} \\
& w_{1}=\omega_{1}^{*}-\frac{2}{a\left(k^{*}+1\right)}\left\{\mathrm{k}^{*} \times \mathrm{g}^{*}-\frac{a}{2} \mathrm{k}^{*} \times\left[\mathrm{k}^{*} \times\left(\omega^{*}+\omega_{1}^{*}\right)\right]\right\},
\end{aligned}
$$

onde

$$
\mathrm{g}^{*}=\mathrm{c}_{1}^{*}-\mathrm{c}^{*}, \quad \mathrm{k}^{*}=-\mathrm{k} \text {. }
$$

Pot outro lado, das equaçóes (1.38), (1.39) e (1.40), temos que

$$
\mathrm{g} \cdot \mathrm{k}=-\mathrm{g}^{*} \cdot \mathrm{k}=\mathrm{g}^{*} \cdot \mathrm{k}^{t}=-\mathrm{g}^{*} \cdot \mathrm{k}=\mathrm{g}^{*} \cdot \mathrm{k}^{*}
$$

\subsubsection{A Equação de Boltzmann}

Una molecula de um gás poliatônico pode ser especificada, em cada instante de tempo $t$, pela posiçắ $\mathrm{x}$ de seu centro de massa, a velocidade linear $\mathrm{c}$ do centro de massa e velocidade angular $w$ em tomo de um eixo que passa pelo centro de massa. Associamos a cada molécula um ponto no espaço 9-dimensional, denominado espaço de fase , determinado por três coordenadas de posição e seis coordenadas de velocidades. Neste espaço, o sistema constituído pelas $N$ moléculas do gás é descrito por $N$ pontos com coordenadas $\left(x_{\alpha}, c_{\alpha}, \omega_{a}\right)$, sendo $\alpha=1,2, \cdots, k$.

$O$ estado do gás no espaço de fase $\mu$ descrito por uma funçâa de distribuiçäo $f(x, c, t, t)$. tal que

$$
\int(x, c, w, t) d x d c d w
$$

nos dá o número de moléculas que se encontram no elemento de volume entre $\mathrm{x}$ e $\mathrm{x}+d \mathrm{x}$, com velocidades lineares entre $\mathrm{c} e \mathrm{c}+d \mathrm{c}$ e velocidades angulares entre $\omega$ e $\omega+t w$, no instante $t$

Vamos considerar que cada molécula do gás, com momento de inércia $I$ e romento angular $\mathbf{L}=1 \omega$, esteja sujeita a uma força específica externa $\mathbf{F}_{m}$ que não depende das velocidades das moléculas, dada por

$$
\mathbf{F}_{m}=\mathbf{F}_{H}+\mathbf{F}_{H}
$$

onde $\mathrm{F}$ tuma forca especifica que pode alterar as velocidates lineares das moleculas fromo a forca gravitacional, por exemplo) e $F_{H} \dot{e}$ uma força específica que pode alterar as velocidades 
angulares das moléculas (por exemplo, em campos magnéticos externos o torque sobre uma molécula é dado por $\tau=I \frac{\text { th }}{\text { it }}$ ).

Portanto, após um intervalo de tempo $\Delta t$ as moléculas ocuparão uma nova posição no espaço de fase, a saber: $x+c \Delta t, c+F \Delta t$ e $\omega+\frac{T}{T} \Delta t$. Podemos entăo afirmar que: no intervalo de tempo entre $t$ e $t+\Delta t$, o número de moléculas que se encontram no elemento de volume entre $x+c \Delta t e(x+s \Delta t)+d x$, com velocidades lineares entre $c+F \Delta t e(c+F \Delta t)+d c$ e com velocidades angulares entre $\omega+\frac{T}{T} \Delta t e\left(\omega+\frac{\tau}{T} \Delta t\right)+d \omega$, é dado por

$$
f\left(\mathrm{x}+\mathrm{c} \Delta t, \mathrm{c}+\mathrm{F} \Delta t, \omega+\frac{T}{l} \Delta t, t+\Delta t\right) d \mathrm{x} d \mathbf{c} d \omega
$$

Os números de moléculas representados pelas equaçues (1.45) e (1.47) seriam iguais se näo houvessem colisôes entre as moléculas. Porém, as colisóes alteram a densidade do número de moléculas no elemento de volume $d x$ de dw desde que algumes moleculas deíxam o elemento de volume enquanto outras, provenientes de outros elementos de volume, entram no mesmo. A mudança do número de moléculas neste elemento de volume propocional ao elemento de volume $\mathbf{k}$ dc $d \omega$, isto é

$$
\left[f\left(\mathrm{x}+\mathrm{c} \Delta t, \mathrm{c}+\mathrm{F} \Delta t, \omega+\frac{\tau}{I} \Delta t, t+\Delta t\right)-f(\mathrm{x}, \mathrm{c}, \omega, t)\right] d \mathrm{x} d \mathrm{c} d \omega=Q d \mathrm{x} d \mathrm{c} d \omega \Delta t
$$

onde $Q$ é a taxa de variação temporal da função de distribuição $f$ devido as colisôes entre as moléculas.

Se dividirmos a equaça (1.48) por $d x d c d \omega$ e passarmos ao limite quando $\Delta t$ tende a zero, obtemos a eçucucio de Boltzman

$$
\frac{\partial f}{\partial i}+c_{i} \frac{\partial f}{\partial x_{i}}+F_{i} \frac{\partial f}{\partial c_{i}}+\frac{\pi_{i}}{I} \frac{\partial f}{\partial \omega_{i}}=Q
$$

A taxa de variaçào temporal $Q$ apresenta duas contribuiçōes, ou seja

$$
Q=Q^{(+)}+Q^{(-)}
$$

onde $Q^{t+1}$ está relucionado ao número de moléculas que entram no elemento de volume $d x d c$ du $\in Q^{(-)}$ao número de moléculas que saem deste volume. Na determinaçäo de $Q$ empregaremos as seguintes hipóteses:

(i) par um gás rarefeito somente as interaçôs entre pares de moléculas (colisôes binarias) săo levadas em consideraçăo;

(ii) durante uma colisão o efeito das forças externas é pequeno quando comparado com o efeito das forças de interaça entre as moleculas:

(iii) as velocidades de duas moléculas, para quasqquer posição e tempo, näo estäo correla. rionadas (hipótese do caos molecular); 


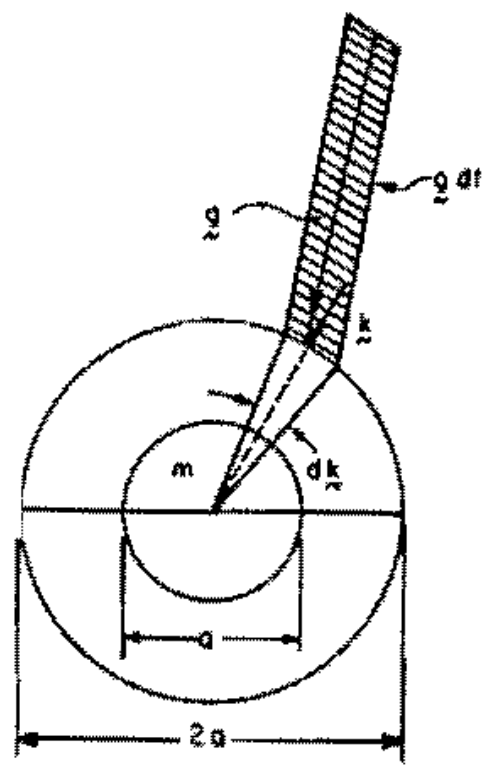

Figura 1.4: Esfera de influêtocia numa colisão binária.

(iv) a função de distribuiçẵo é considerada constante ao longo de distăncias comparáveis ao tamanho da molécula porém, näo ao longo de distâncias comparáveis ao livre caminho médio.

Cálculo de Q. Para calcular a taxa de colisöes $Q$ observemos inticialmente que, no instante da colisão. o centro da molécula 1 esta sobre a superficie de uma esfera de raio a, centrada na moltcula sem rótulo, que denominamos de esfera de inliuência (veja figura 1.4); a condigà̃o $\mathrm{g} \cdot \mathbf{k}>0$ deve ser verificada pois assegura que a molécula 1 se desloca na direção da molécula sem rốtulo.

Constderemos as colisôes entre moleculas com velocidades entre $(c, \omega)$ e $(c+t c, \omega+d \omega)$ com moléculas com velocidades entre $\left(c_{1}, \omega_{1}\right)$ e $\left(c_{1}+d c_{1}, \omega_{1}+d \omega_{1}\right)$ e direçöes da linha que une os centros das moléculas entre $\mathrm{k} e \mathrm{k}+d \mathrm{k}$. Podemos, de ângulo sólido subentendido por uma esfera $\operatorname{com}$ raio unitário $|\mathrm{k}|=1$, ou seja, $d \mathrm{k}=d \Omega=$

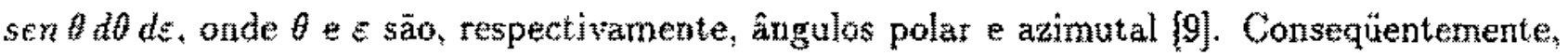
no intervalo de tempo entre $t e t+\Delta t_{z}$ todas as moléculas cujos centros estiverem dentro do cilindro com area de base $a^{2} d \Omega=a^{2} d \mathrm{k}=a^{2} \operatorname{sen} \theta d \theta d c$ altura $(\mathrm{g} \cdot \mathrm{k}) d \mathrm{~d}$, irăo colidir com a molécula com centro en $x$.

Portanto. o nímero de colisóes que ocorrem no elemento de volume $d x$ e no intervalo de lempo dt. é dado por 


$$
\underbrace{\int(\mathrm{x}, \mathrm{c}, \omega, t) d \mathrm{x} d \mathrm{c} d \omega}_{(x)} \underbrace{\int\left(\mathrm{x}, \mathrm{c}_{1}, w_{1}, t\right) a^{2}(\mathrm{~g} \cdot \mathrm{k}) d \mathrm{k} d \mathrm{c}_{1} d \omega_{1} d t}_{(\mathrm{b})}
$$

Nesta expressăo, o fator (a) representa o número de moléculas com velocidades entre $(c, \omega)$ e $(\mathrm{c}+d \mathrm{c}, \omega+d \omega)$ que se encontram no elemento de wolume entre $\mathrm{x} \in \mathrm{x}+d \mathrm{x}$, enquanto que 0 fator (b) representa o número de moléculas com velocidades entre $\left(c_{1}, \omega_{1}\right)$ e $\left(c_{1}+d c_{1}, \omega_{1}+d w_{1}\right)$ que se encontram no cilindro com volume $a^{2}(\mathrm{~g}+\mathrm{k}) \mathrm{dk} d t$.

Se dividirmos a equaça (1.51) por $d x d c d w d t$ e integrarmos sobre todas as velocidades $c_{1}$ e $w_{1}$ e sobre todas as direçotes de $\mathbf{k}$, obteremos a densidade do número total de colisoes por intervalo de tempo dt que anulam pontos de fase con velocidades $\mathrm{c} e \omega$ no elemento $d \mathrm{x} d \mathrm{c} d \omega$ do espaço de fase, ou seja

$$
Q^{(-)}=\int f\left(x_{1}, \omega_{2}, t\right) f_{1}\left(x_{2}, c_{1}, \omega_{1}, t\right) a^{2}(g+k) d k d c_{1} d \omega_{1}
$$

Por outro lado, a criaça de pontos de fase com velocidades $(c, \omega)$ no espaço de fase ocorre através de colisôes de restituição, ou seja, das colisōes entre moléculas corn velocidades ( $\mathrm{c}^{*}, \omega^{*}$ ) com moléculas com velocidades $\left(c_{1}^{*}, \omega_{1}^{*}\right)$ e vetor de colisäo $k^{*}=-k$; a densidade do número destas colisồes, no intervalo de tempo d, tem forma análoga a da equaçăo (1.51), isto é

$$
f\left(\mathrm{x}_{*} \mathrm{c}^{*}, \omega^{*}, t\right) d \mathrm{x} d \mathrm{c}^{*} d \omega^{*} f\left(\mathrm{x}, \mathrm{c}_{1}^{*}, \omega_{1}^{*}, t\right) a^{2}\left(\mathrm{~g}^{*} \cdot \mathrm{k}^{*}\right) d \mathrm{k}^{*} d \mathrm{c}_{1}^{*} d \omega_{1}^{*} d t
$$

com $\mathrm{g}^{*} \mathrm{e} \mathrm{k}^{*}$ definidos pela equaçäo (1.43).

A relaçäo entre elementos de volume no espaço das velocidades é dada por

$$
d c d \omega d c_{1} d \omega_{1}=|N| d c^{*} d \omega^{*} d c_{1}^{*} d \omega_{1}^{*}
$$

onde $J$ te o determinante jacobiano da transformaça, definido por

$$
J=\frac{\partial\left(c, w_{1} c_{1}, \omega_{1}\right)}{\partial\left(\mathrm{c}^{*}, w^{*}, c_{i}^{*}, w_{1}^{*}\right)}
$$

Com as equaçôes de transformação (1.39) a (1.43) obtemos que $|J|=1$; assim, a equuaçăo $(1,34)$ torna-se

$$
d \mathrm{c} d \omega d \mathrm{c}_{1} d \omega_{1}=d \mathrm{c}^{*} d \omega^{*} d \mathrm{c}_{i}^{*} d \omega_{1}^{*}
$$

\footnotetext{
'Nesta equastuo o simbolo de integraz̧ăo representa oito integrais correspondentes a: trés componentes da

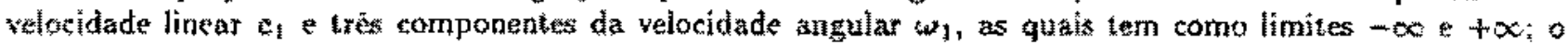

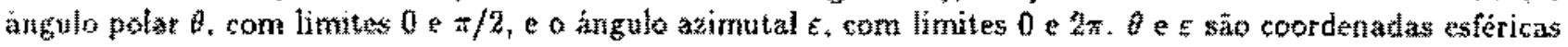
para o versor $\mathbf{k}$.
} 
de modo que a equação (1.53) pode ser escrita como

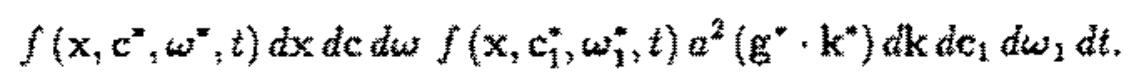

Dividindo esta equaçấo por $d x d \mathrm{c} d \omega \mathrm{dt}$, considerando da equaçäo (1.44) que $\mathrm{g}^{*} \cdot \mathrm{k}^{*}=\mathrm{g} \cdot \mathrm{k}$ e integrando sobre todas as velocidades e sobre todas as direçốes possiveis de colisâo, obtemos

$$
Q^{(+)}=\int f\left(\mathrm{x}, \mathrm{c}^{*}, \omega^{*}, t\right) f_{1}\left(\mathrm{x}, \mathrm{c}_{1}^{*}, \omega_{1}^{*}, t\right) a^{2}(\mathrm{~g} \cdot \mathrm{k}) d \mathrm{k} d \mathrm{c}_{1} d \omega_{1}
$$

que representa a densidade do mumero de colisóes, por intevalo de tempo $d t$, que cria pontos de fase com velocidades $(\mathrm{c}, \omega)$ no elemento do espaco de fase $d x \mathrm{de} d \omega$.

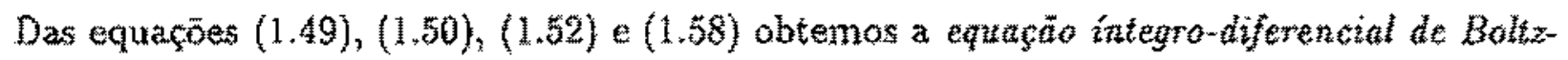
mann, na forma

$$
\frac{\partial f}{\partial t}+c_{i} \frac{\partial f}{\partial x_{i}}+F_{i} \frac{\partial f}{\partial c_{i}}+\frac{r_{i}}{f} \frac{\partial f}{\partial \omega_{i}}=\int\left(f_{1}^{*} f^{*}-f_{1} f\right) a^{2}(\mathrm{~g} \cdot \mathrm{k}) d \mathrm{k} d \mathrm{c}_{1} d \omega_{1}
$$

Nesta equaçäo introduzimos as seguintes abreviaçö̌s:

$$
\begin{array}{ll}
f^{*}=f\left(x, c^{*}, \omega^{*}, t\right), & f=f\left(x, c_{3}, \omega_{1} t\right) \\
f_{1}^{*}=f\left(x, c_{1}^{*}, \omega_{1}^{*}, t\right), & f_{1}=f\left(x, c_{1}, \omega_{1}, t\right),
\end{array}
$$

sendo que os termos com asterisco denotam colisôes de restiłuiçăo.

$\dot{E}$ conveniente ressaltar que na equação (1.59) as funçöes de distribuição são calculadas no mesmo ponto $x$ no instante da colisăo. Isto reflete o fato de que para um gás rarefeito não se consideram as dimensōes das moléculas.

\subsubsection{A Equaçāo de Transporte e os Invariantes de Soma}

Para obtermos a equaçäo de transporte iniciamos multiplicando a equaçäo de Boltzmann (1.39) por uma funçäo arbitráría $\varphi\left(x_{1}, c, \omega, t\right)$ e integrando a equaçăo resultante em todos os valores das velocidades $c \omega$, ou seja

$$
\int \psi\left(\frac{\partial f}{\partial t}+c_{i} \frac{\partial f}{\partial x_{i}}+F_{i} \frac{\partial f}{\partial c_{i}}+\frac{\tau_{i}}{I} \frac{\partial f}{\partial \omega_{i}}\right) d \mathrm{c} d \omega=\int \varphi\left(f_{i} f^{*}-f_{1} f\right) a^{2}(\mathrm{~g} \cdot \mathrm{k}) d \mathrm{k} d \mathrm{c} d \omega d \mathrm{c}_{1} d \omega_{1}
$$

A equação anterior pode tambẻm, ser escrita como

$$
\begin{aligned}
& \frac{\partial}{\partial t} \int \varphi_{\gamma} \int \mathrm{c} d \omega+\frac{\partial}{\partial x_{i}} \int c_{\varphi} \int d \mathrm{c} d \omega-\int\left(\frac{\partial \varphi}{\partial t}+c_{i} \frac{\partial \varphi}{\partial x_{i}}+F_{i} \frac{\partial \varphi}{\partial c_{i}}+\frac{r_{i}}{I} \frac{\partial \varphi}{\partial \omega_{i}}\right) \int d \mathrm{c} d \omega= \\
& =\int \rho\left(f_{1}^{*} f^{*}-\int f\right) a^{2}(g \cdot k) d k d c d w d c_{1} d \omega_{1}
\end{aligned}
$$


onde consideramos que as velocidades moleculares säo independentes da posiçào e do teorema de Gauss, que

$$
\begin{aligned}
& \int \frac{\partial}{\partial c_{i}}\left(\varphi F_{i} f\right) d c=\oint\left(\varphi F_{i} f\right) n_{i}^{c} d S_{c}=0 \\
& \int \frac{\partial}{\partial \omega_{i}}\left(\varphi r_{i} f\right) d \omega=\oint\left(\varphi r_{i} f\right) n_{i}^{\omega} d S_{t u}=0
\end{aligned}
$$

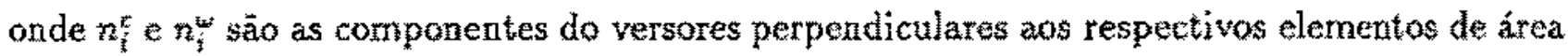
$d S_{0}$ e $t S_{w}$ do espaço de velocidades. Ambas as integrais são nulas pois a funçäo de distribuiçăo decai rapidamente a zero para grandes valores das velocidades.

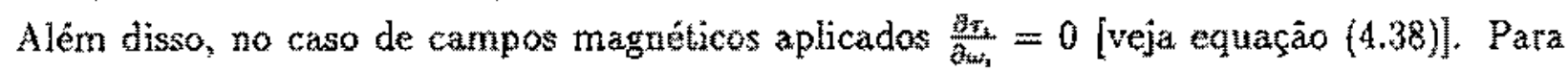
transformat o lado diretto da equaçäo (1.62) consideremos que, das equações (1.28) a (1.31) e (1.56), temos que

$$
d c d \omega d \mathrm{c}_{1} d \omega_{1}=d \mathrm{c}^{2} d \omega^{*} d \mathrm{c}_{1}^{\prime} d \omega_{1}^{\prime}=d \mathrm{c}^{*} d \omega^{*} d \mathrm{c}_{1}^{*} d \omega_{1}^{*}
$$

Desta lorma, da equacta (1.44) podemos escrever que

$$
\begin{aligned}
& \left.\int \psi(\mathrm{x}, \mathrm{c}, \omega, t) f_{1}^{*}\right)^{*} a^{2}(\mathrm{~g} \cdot \mathrm{k}) d \mathrm{k} d \mathrm{c} \omega \omega^{2} d \mathrm{c}_{1} d \omega_{1} \\
& =\int \varphi\left(\mathrm{x}, \mathrm{c}, \omega^{*}, t\right) f_{1}^{*} f^{*} a^{2}\left(\mathrm{~g}^{*} \cdot \mathrm{k}^{*}\right) d \mathrm{k}^{*} d \mathrm{c}^{*} d \omega^{*} d \mathrm{c}_{\mathrm{I}}^{*} d \omega_{1}^{*} \\
& =\int \varphi\left(\mathrm{x}, \mathrm{c}^{\prime}, \omega^{\prime}, t\right) \mathrm{h} \int \mathrm{a}^{2}(\mathrm{~g}, \mathrm{k}) d \mathrm{k} d \mathrm{c} d \omega d \mathrm{c}_{1} d \omega_{1},
\end{aligned}
$$

onde a ultima igualdade resulta da observaçäo de que $\left(\mathrm{c}^{*} ; \omega^{*} ; \mathrm{c}_{1}^{*}, w_{1}^{*}\right)$ representam as velocidades iniciass correspondentes às velocidades finais $\left(c, w ; c_{1}, \omega_{3}\right)$, com a direça da linha que une os centros dada por $k^{*}=-k_{i}$ apenas trocamos a notação e adotamos $\left(c_{1} \omega_{i} c_{1}, \omega_{1}\right)$ para as

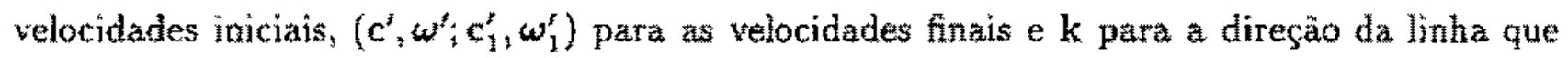
une os centros. Porbanto, para o lado direito da equaçăo (1.62), temos

$$
\begin{aligned}
& \int \varphi\left(f_{\mathrm{i}}^{*} f^{*}-f_{1} f\right) a^{2}(\mathrm{~g} \cdot \mathrm{k}) \mathrm{k} d \mathrm{c} d \omega d \mathrm{e}_{1} d \omega_{3}=\int\left(\varphi^{\prime}-\varphi_{1} f a^{2}(\mathrm{~g} \cdot \mathrm{k}) d \mathrm{k} d \mathrm{c} d \omega d \mathrm{c}_{1} d \omega_{1}=\right. \\
& =\int\left(\varphi_{1}^{\prime}-\varphi_{1}\right) f_{1} \int \alpha^{2}(\mathrm{~g} \cdot \mathrm{k}) \mathrm{dk} d \mathrm{~d} d \omega \mathrm{dc_{1 }} d \omega_{1} \text {. }
\end{aligned}
$$

Para obter a ultima igualdade da equaçăo anterior mudamos o papel das moleculas que colidem. isto consideramos moléculas con velocidades niciais $\left(c_{1}, \omega_{1} ; c, \omega\right)$ e velocidades fintis $\left(\mathrm{c}_{1}^{p}, \omega_{1}^{z}: \mathbf{c}^{\prime} \cdot \omega^{\prime}\right)$; consequentemente, trocamos $\mathrm{k}$ por $-\mathrm{k}$ e $\mathrm{g}$ por $-\mathrm{g}$.

Com a equą̧̃̃ (1.66) podemos reescrever a (1.62) na forma 


$$
\begin{aligned}
& \frac{\partial}{\partial t} \int \varphi \int d \mathrm{c} d \omega+\frac{\partial}{\partial x_{i}} \int c_{i} f d \mathrm{c} d \omega-\int\left(\frac{\partial \varphi}{\partial t}+c_{i} \frac{\partial \varphi}{\partial x_{i}}+F_{i} \frac{\partial \varphi}{\partial c_{i}}+\frac{T_{i}}{T_{\varphi}} \frac{\partial \varphi}{\partial \omega_{i}}\right) f d \mathrm{c} d \omega \\
& =\int \varphi\left(f_{1}^{*} f^{*}-f_{1} f\right) a^{2}(\mathrm{~g} \cdot \mathrm{k}) d \mathbf{k} d \mathrm{c} d \omega d \mathbf{c}_{1} d \omega_{1}= \\
& =\frac{1}{2} \int\left(\varphi_{1}^{2}+\varphi^{t}-\varphi_{1}-\varphi\right) a^{2} f_{1} f(\mathrm{~g} \cdot \mathrm{k}) d \mathrm{k} d \mathrm{c} d \omega \mathrm{d} \mathrm{c}_{1} d \omega_{1}
\end{aligned}
$$

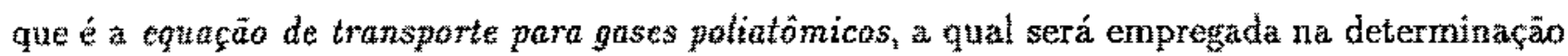
das equaçōes de balanço para as propriedades macroscópicas do gás.

A equaçäo (1.67) pode ser escrita na forma abreviada

$$
\frac{\partial \Psi}{\partial t}+\frac{\partial}{\partial x_{i}}\left(\psi v_{i}+\phi_{i}\right)=S+p
$$

onde $\Psi$ è a densidade de uma quantidade aditiva arbitrária, $v_{i}$ é a velocidade hidrodinamica do gás, $\phi_{i}$ a sua densidade de fluxo, $S$ a densidade de suprimento relacionada à forças e torques externos e $P$ o termo de produçăo. Essás quantidades săo definidas por:

$$
\begin{aligned}
& \Psi=\int \cos d \mathrm{c} d \omega \\
& \varphi=\int \varphi C_{i}{ }^{2} d e d \omega \\
& S=\int\left(F_{i} \frac{\partial \varphi}{\partial c_{i}}+\frac{\pi_{i}}{I} \frac{\partial \varphi}{\partial \omega_{i}}\right) f d \mathrm{c} d \omega \\
& P=\int\left(\frac{\partial \varphi}{\partial t}+c_{i} \frac{\partial \varphi}{\partial x_{i}}\right) \int d \mathrm{c} d \omega+\frac{1}{2} \int\left(\varphi_{1}^{*}+\varphi^{\prime}-\varphi_{1}-\varphi\right) f \int_{1} a^{2}(\mathrm{~g} \times \mathrm{k}) d \mathrm{k} d \mathrm{c} d \omega d \mathrm{c}_{1} d \omega_{1}
\end{aligned}
$$

onde $C_{i}=c_{i}-v_{i}$ é a velocidade peculiar da molécula.

Uma grandeza $\psi$ que se conserva numa colisão, isto $e_{\text {q }}$ que satisfaz

$$
\dot{\psi}+\psi_{1}=\psi^{z}+\psi_{1}^{\prime}
$$

e denominada um invariante de soma. Para estas grandezas o termo de colisão da equação de transporte (1.67) é nuto. No caso de gases poliatomicos, os invariantes de soma para um par de molêtulas, são:

- a massa

$$
\xi^{4}=m \text {. }
$$


- o momento linear

$$
\psi^{(2)}=m \mathbf{c},
$$

- a energia total

$$
\psi^{(3)}=\frac{1}{2} m c^{2}+\frac{1}{2} I \omega^{2}
$$

- o momento angular total

$$
\psi^{(4)}=I \omega+\mathbf{x} \times(m c)
$$

onde $\mathrm{x}$ é o vetor posição do centro de massa da molécula. O invariante de soma $\psi^{(4)}$ é importante quando tratamos do gás denso de Enskog [9] para o qual as posições das moléculas são consideradas como distintas numa colisão. Este não é o caso dos gases rarefeitos, que constituem o objeto do nosso estudo. 


\section{Capitulo 2}

\section{Descrição Macroscópica de um Gás Ideal Poliatômico}

\subsection{Introdução}

Neste capitulo apresentamos uma descrição macroscópica de gases ideás poliatônicos a partir dos conceitos microscópicos da teoria cinética.

Do ponto de vista da termodinámica um gás ideal poliatômico pode ser descrito atraves de cinco campos básicos denominados de campos da termodinâmica. Estes campos satistarem a equaçóes de balanço que podem ser obtidas atrayés da teoria cinéticta. Para tornar este conjunto de equaçoes um sistema fechado novas grandezas, denominadas de grandezas constitutivas, devem ser determinadas.

Verificamos que, da equaça de balanço para a densidade de entropia, decorre uma desigual. dade que explicita o fato de que a produçăo de entropia é uma grandeza näo negativa. Esta

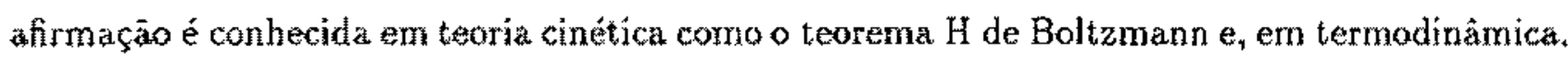
como a segunda lei da termodinârnica.

A funçăo de distribuiçăo para o estado de equilíbrio de urn gás poliatômico é determinada através de um método anälogo ao que conduz à funçào de distribuição maxwelliana para gases monoatômicos isto é, tornando nulo o termo de colista na equação de Boltzmann.

Através da equação de Boltzmann concluimos que um gás poliatômico no estado de equiltbrio é regido pelas equaçóes de balanço para um fluido de Euler, com as condiçoes adicionais de qute o gradiente de temperatura e o gradiente da velocidade hidrodinâmica sä̊ nulos.

\subsection{Campos Macroscópicos Básicos}

O estado macroscópico de um gás poliatómico pode ser descrito através de grandezas denominadas enmpos da lennodinânica. Descreveremos o gấs em termos de cinco cantpos escalares basicos, os quais sāo: densidade de massa $\varrho(x, b)$, densidade de momento linear $g v_{i}(x, 1)$ 
e densidade de energia $\varrho u(x, t)$.

Em teoria cinética estes campos são definidos como médias, em relaçäo a funçăo de distribuiçẫo $/(x, c, \omega, t)$, das grandezás microscópicas que são invariantes de soma, ou seja

$$
\begin{gathered}
e=\int m \int d c d w \\
\varrho^{v_{i}}=\int m c_{n} f d c d w \\
\varrho^{u}=\int\left(\frac{1}{2} m c^{2}+\frac{1}{2} \int w^{2}\right) \int d \mathrm{c} d \omega
\end{gathered}
$$

Consideraremos que o gás não apresenta rotação como um todo, de modo que a densidade de spin, definida por

$$
e \sigma_{i}=\int I w_{i} f d \mathbf{c} d \omega
$$

é nula.

Introduzimos a velocidade peculiar de translação para uma molécula do gás, por

$$
C_{i}(x, t)=c_{i}-v_{i}(x, t)
$$

que representa a velocidade da molécula no ref́terencial do gas em repouso.

De forma anäloga, a velocidade peculiar de rotaçác é definida por

$$
\Omega_{i}(x, t)=w_{i}-s_{i}(x, t)
$$

onde $s_{i}=\frac{m}{7} \sigma_{i}$ é a velocidade de spin. Para gases que não apressentam rotaçẫo como um todo de nodo que $\sigma_{\mathrm{i}}=0_{\mathrm{i}} \mathrm{e}$

$$
\Omega_{i}=w_{i}
$$

Se substituirmos a equagào (2.5) na (2.2) e, tendo em conta a equaçäo (2.1), verificamos que a velocidade peculiar mểia é nula, ou seja

$$
\int m C_{i} f d c d w=0
$$

Ao substituirmos as velocitades peculiares (2.5) e (2.7) nă equação (2.3) e considerando a equação (2.8), obtemos para a densidade de energia

$$
\rho_{u}=g \varepsilon+\frac{1}{2} g v^{2}
$$

onde definimos a densidade de energia interna do gás por

$$
g^{z}=\int\left(\frac{1}{2} m C^{2}+\frac{1}{2} I \Omega^{2}\right) \int d c d w
$$


Assim, na equaçăo (2.9) o termo $\frac{1}{2} \varrho v^{2}$ representa a densidate de energia cinatica do gás como un todo.

O momento de orden $N$ da funçüo de distribuiçă, é definido por

$$
p_{i_{1}, i_{2}, \cdots, i_{i}}(\mathrm{x}, t)=\int m C_{i_{1}} C_{i_{2}} \cdots C_{\xi_{N}} f(\mathrm{x}, \mathrm{c}, \omega) d \mathrm{c} d \omega
$$

e representa um tensor simétrico de ordem $N$ com $\frac{1}{2}(N+1)(N+2)$ componentes distintas.

0 momento de ordem zero é a densidade de massa $g$ enquanto que o primeiro momento é nulo como consequência da equação (2.8). O segundo momento

$$
p_{i j}=\int m C_{i} C_{j} f(x, c, w) d e d w
$$

é denominado de tensor pressão e csta relacionado ao tensor tensäo $t_{i j}$ por $p_{i j}=-t_{i j}$

\subsection{Equações de Balanço para Campos Macroscópicos}

As equaçós de balanço para os campos macroscópicos densidade de massa $\rho_{\text {a }}$ densidade de momento linear $g v_{i}$ e densidade de energiz $\rho^{u}$ são obtidas ao igualarmos a funçäo escalar $p(x, c, t)$, sucessivamente, aos invariantes de soma: massa $m$, momento linear $m c_{i}$ e energia $\frac{1}{2} m c^{2}+\frac{1}{2} I \omega^{2}$ na equaça de transporte (1.67). Após realizarmos as integraçöes, obtemos:

- bilanço de densidade de massa: $\varphi=m$

$$
\frac{\partial e}{\partial t}+\frac{\partial \varrho v_{i}}{\partial x_{i}}=0
$$

- balango de densidade de momento linear: $p=$ mo

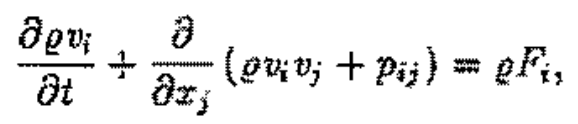

- balanço de densidade de energia: $p=\frac{1}{2} m c^{2}+\frac{1}{2} f w^{2}$

$$
\frac{\partial}{\partial t}\left[g\left(\epsilon+\frac{1}{2} v^{2}\right)\right]+\frac{\partial}{\partial x_{i}}\left[\hat{g}\left(c+\frac{1}{2} v^{2}\right) w_{i}+q_{i}+p_{i j} v_{j}\right]=\partial F_{i} z_{i}
$$

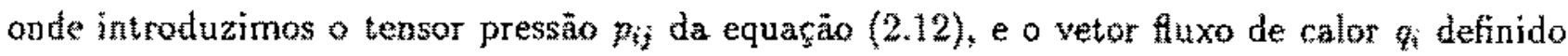
por

$$
q_{t}=\int\left(\frac{1}{2} m C^{2}+\frac{1}{2} l \Omega^{2}\right) C_{i} \hat{f} d c d \omega
$$


Se multiplicarmos escalarmente a equação (2.14) por tu abtraimos o resultado da (2.15), obtemos a equação de balanço para a energia especifica interna:

$$
\frac{\partial \varepsilon}{\partial t}+\varrho \frac{\partial \varepsilon}{\partial x_{i}}+\frac{\partial q_{i}}{\partial x_{i}}+p_{i j} \frac{\partial u_{i}}{\partial x_{j}}=0
$$

Notemos que as equaçóes de balanço (2.13) a (2.15) näo constituem um sistema fechado de equacoos; para tanto é necessário conhecer as equaçôes constitutivas para o tensor pressäo $p_{i j}$ e para o vetor fluxo de calor $q_{i}$ em termos dos campos básicos. Tais equaçöes dependem do meio a que se referem e são denominadas de equaçôes constitutivas para o meio considerado. A determinaçio das equaçōes constitutivas torna-se possivel desde conheçamos a funçăo de distributcato $f(x, c, \omega, t)$, como podemos verificar dus equaçoes $(2.12)$ e $(2.16)$.

\subsection{Entropia}

Consideremos função

$$
\varphi(x, c, w, b)=-k \ln (b f)
$$

onde $k$ é a constante de Boltzmann e $b$ uma constante arbitrária. Substituindo na equaçẫo de transporte (1.67), obtemos

$$
\frac{\partial \varrho s}{\partial t}+\frac{\partial}{\partial x_{i}}\left(\varrho s v_{i}+\phi_{i}\right)-k \int\left(\frac{\partial f}{\partial t} c_{i}+\frac{\partial f}{\partial x_{i}}+F_{i} \frac{\partial f}{\partial c_{i}}+\frac{\tau_{i}}{I} \frac{\partial f}{\partial \omega_{i}}\right) d \mathbf{c} d \omega=\sigma
$$

onde introduzimos a seguinte notaçăo:

$$
\begin{aligned}
& \left.\rho s=-\iint \ln (b)\right) d c t u \\
& \phi_{i}=-n \int \ln (b f) G_{i} d c d \omega \\
& \sigma=-\frac{k}{2} \int \ln \left(\frac{f_{1} f}{f_{1} f}\right) f_{1} f a^{2}(\mathrm{~g}-\mathrm{k}) d \mathrm{k} d c_{1} d \omega_{1} d \mathrm{c} d \omega
\end{aligned}
$$

Podemos verificar que o termo da equaçầ (2.19) que contêm a integral é rulo. De fato; tomando $\varphi=1$ nas equaçōes (1.61) e (1.66), obtemos:

$$
\int\left(f_{i}^{*} f^{*}-f f\right) a^{2}(\mathbf{g} \cdot \mathbf{k}) d \mathbf{k} d c_{1} d \omega_{1} d \mathrm{c} d \omega=\int\left(\frac{\partial f}{\partial t}+c_{i} \frac{\partial f}{\partial x_{i}}+F_{i} \frac{\partial f}{\partial c_{i}}+\frac{T_{i}}{I} \frac{\partial f}{\partial \omega_{i}}\right) d \mathrm{c} d \omega=0
$$

Desta forma, da equaça $(2.19)$ obtemos a seguinte equaçào de balanço:

$$
\frac{\partial \rho}{\partial t}+\frac{\partial}{\partial x_{i}}\left(g s m_{i}+\phi_{i}\right)=\sigma
$$


Vamos explorar um pouco mais o termo o definido pela equaçzo (2.22). Se escolhermos $\varphi=1$ na equaço $(1.66)$, temos

$$
\begin{gathered}
\int f_{1}^{*} f^{*} a^{2}(\mathrm{~g} \cdot \mathrm{k}) d \mathrm{k} d \mathrm{c}_{1} d \omega_{1} d \mathrm{c} d \omega=\int f_{1} f a^{2}(\mathrm{~g} \cdot \mathrm{k}) d \mathrm{k} d \mathrm{c}_{1} d \omega_{1} d \mathrm{c} d \omega \\
=\int f_{1}^{*} f^{*} a^{2}\left(\mathrm{~g}^{*} \cdot \mathrm{k}^{*}\right) d \mathrm{k}^{*} d \mathrm{c}_{1}^{*} d \omega_{1}^{*} d \mathrm{c}^{*} d \omega^{*}
\end{gathered}
$$

sendo que a segunda igualdade segue das equaçōe (1.44) e (1.64).

Na equaçäo (2.25) as grandezas com e sem asterisco se referem a uma colisäo binária de moléculas cujas velocidades iniciais foram denotadas por $\left(c_{1}^{*}, \omega_{1}^{*} ; c^{*}, \omega^{*}\right)$ e velocidades finais por $\left(\mathrm{c}_{1}, \omega_{1} ; c, \omega\right)$, com o vetor de colisão $\mathrm{k}^{*}=-\mathrm{k}$. Se mudarmos os símbolos e denotarmos as velocidade iniciais por $\left(c_{1}, \omega_{1} ; c_{i} \omega\right)$, as velocidades finais por $\left(c_{1}^{\prime}, \omega_{1}^{\prime} ; c^{\prime}, \omega^{\prime}\right)$ com $k^{\prime}=-k$ para o vetor de colisão, segue para a segunda igualdade da equação (2.25):

$$
\int f_{1}^{t} \int^{t} a^{2}\left(\mathrm{~g}^{t} \cdot \mathrm{k}^{\prime}\right) d \mathrm{k}^{t} d \mathrm{c}_{1}^{t} d \omega_{1}^{t} d \mathrm{c}^{t} d \omega^{\prime}=\int f f a^{2}(\mathrm{~g} \cdot \mathrm{k}) d \mathrm{k} d \varepsilon_{1} d \omega_{1} d \mathrm{c} d \omega
$$

e com base nas equaçós $(1.44)$ e $(1.64)$, podemos escrever que

$$
\int f_{1} f^{2}(\mathrm{~g} \cdot \mathrm{k}) d \mathrm{k} d \mathrm{c}_{1} d \omega_{1} d \mathrm{c} d \omega=\int f_{1} f a^{2}(\mathrm{~g} \cdot \mathrm{k}) d \mathrm{k} d \mathrm{c}_{1} d \omega_{1} d \mathrm{c} d \omega
$$

A equaçào anterior tern a forma squivalente

$$
\frac{k}{2} \int\left(\frac{f_{1}^{\prime} f^{\prime}}{f_{1} f}-1\right) f_{1} f a^{2}(\mathrm{~g} \cdot \mathrm{k}) d \mathrm{k} d \mathrm{c}_{1} d \omega_{1} d \mathrm{c} d \omega=0
$$

de modo que a equação $(2.22)$ pode ser escrita como

$$
g=-\frac{k}{2} \int\left[\ln \left(\frac{f_{1}^{\prime} f^{\prime}}{f_{1} f}\right)-\left(\frac{f_{1}^{\prime} f^{\prime}}{f_{1} f}-1\right)\right] f_{1} f a^{2}(\mathrm{~g} \cdot \mathrm{k}) d \mathrm{k} d \mathrm{c}_{1} d \omega_{1} d \mathrm{c} d \omega
$$

Desde que para qualquer $x>0$, temos

$$
\ln x-(x-1) \leq 0
$$

entäo

$$
\sigma=-\frac{k}{2} \int\left[\ln \left(\frac{f_{1}^{\prime} f}{f_{1} f}\right)-\left(\frac{f_{1}^{\prime} f^{\prime}}{f_{1} f}-1\right)\right] f_{1} f^{2}(\mathrm{~g} \cdot \mathrm{k}) d \mathrm{k} d \mathrm{c}_{1} d \omega_{1} d \mathrm{c} d \omega \geq 0
$$

Segue das equacoos $(2.24)$ e $(2.31)$ que

$$
\frac{\partial \rho s}{\partial i}+\frac{\partial}{\partial r_{i}}\left(\operatorname{los} x_{i}+\phi_{i}\right) \geq 0
$$


que representa a equação de balanço para a densidade de entropia gs com o termo de produçào o năo negativo; o termo $\phi_{i}$ é identificado com o fluxo de entropia. Esta equação está em acordo com a segunda lei da termodinämica a qual estabelece que a produção de entropia é nula (sistema em equilíbrio) ou é positiva (sistema fora do equilibrio). $O$ fato da produçäo de entropia ser näo negativa é conhecido em teoria cinética como o leorema $H$ de Boltamann. Lma prova do teorema H para gases poliatômicos clássicos com moléculas apresentando estruturas complexas, é encontrada em [42].

\subsection{Função de Distribuição para o Estado de Equilíbrio}

Em teoria cinética o estado de equilbrio é definido como processo que torna nulo o termo da equaçâo de Boltzmann (1.59) devido às colisóes, isto é,

$$
f_{1}^{(0)} f^{(0)}=f_{1}^{(0)} f^{(0)}
$$

ou

$$
\operatorname{In} f_{1}^{(0)}+\ln f^{(0)}=\ln f_{1}^{(0)}+\ln f^{(0)}
$$

onde o superescrito (0) denota o estado de equilibrio.

Observamos aue a equaçäo (2.34) tem a forma da (1.73), de modo que In $f^{(0)}$ to um invariante de sona assim. a equaçăo (2.34) pode ser escrita como uma combinaçầ linear de termos das equaçòes $(1.74)$ a $(1.76)$, ou seja

$$
\ln f^{(0)}=A+\mathbf{B} \cdot(m c)+D\left(\frac{1}{2} m c^{2}+\frac{1}{2} I \omega^{3}\right)
$$

E conveniente escrevermos os coeficientes indeterminados como

$$
A=\ln a-m b u_{i}^{2}, \quad \mathbf{B}=2 b \mathbf{u}_{\mathbf{1}} \quad \text { e } \quad D=-2 b
$$

sendo $a, b$ e $u_{1}$ novos parânetros a determinar. Portanto, podemos escrever a equação (2.35) como

$$
f^{(0)}=a e^{-m b\left(c-u_{1}\right)^{*}} e^{-1 t w w^{2}}
$$

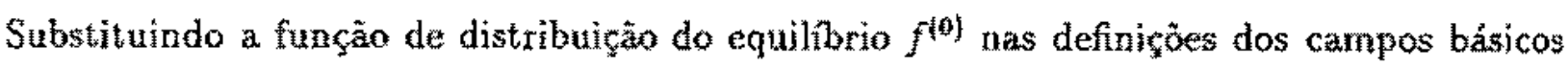
densidade de messa (2.1), densidade de momento linear (2.2), e integrando sobre todas as 
velocidades, obtemos:

$$
p=\int m f^{f 0)} d c d \omega=a m \int e^{-m b\left(c-u_{1}\right)^{3}} d c \int e^{-1 b \omega^{2}} d \omega=a m\left(\frac{\pi}{m b}\right)^{3 / 2}\left(\frac{\pi}{1 b}\right)^{3 / 2}
$$

ou seja

$$
a=\frac{e}{m}\left(\frac{m b}{\pi}\right)^{3 / 2}\left(\frac{m b}{\pi}\right)^{3 / 2}
$$

$\mathbf{e}$

$$
\varrho w_{i}=\int m c_{3} f^{(0)} d \mathrm{c} d \omega=\int m C_{i} f^{(0)} d \mathrm{c} d \omega+u_{1 i} \int m f^{(0)} d \mathrm{c} d \omega=g u_{1}
$$

desde que a primeira integral no lado direito da segunda igualdade é nula. Assim,

$$
u_{1 i}=w_{i}
$$

Com as equacöos (2.5) (2.39) e (2.41) podemos escrever para a função de distribuição (2.37):

$$
f^{f 01}=\frac{g}{m}\left(\frac{m b}{m}\right)^{3 / 2}\left(\frac{m b}{\pi}\right)^{3 / 2} e^{-m b C^{2}} e^{-m n^{2}}
$$

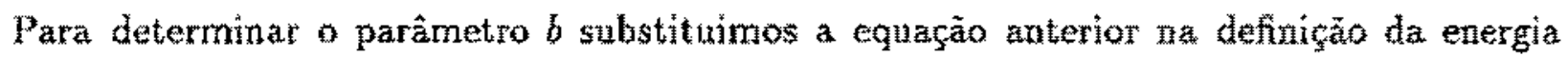
especifica interna (2.10) e integramos sobre todass as velocidades ce wi entäo, obtemos:

$$
\varrho \varepsilon=\int\left(\frac{1}{2} m C^{2}+\frac{1}{2} I \Omega^{2}\right) d \mathbf{c} d \omega=\frac{3}{2} \frac{\varrho}{m} \frac{1}{b}
$$

e, portanto

$$
b=\frac{3}{2} \frac{1}{m \epsilon}
$$

Como cada molécula do gás possui seis graus de liberdade ftrês translacionais e três rotacionais) temos, de acordo com o principio da equipartiça da energia, que a energia especifica por molécula é dada por

$$
\varepsilon=3 \frac{k}{m} T
$$

e segue, das duas últimas equuaçōes, que

$$
b=\frac{1}{2 k T}
$$

Assim. a funçào de distributçäo do equilibrio (2.37) é escrita como

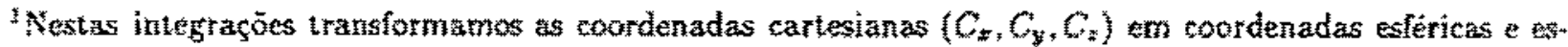
atevernos a integral em at $c=d C_{\text {, como }}$

$$
\int d C=\int_{-\infty}^{+\infty} \int_{-\infty}^{+\infty} \int_{-\infty}^{+\infty} d C_{x} d C_{3} d C_{x}=\int_{0}^{\infty} C^{2} d C \int_{0}^{*} \operatorname{sen} \theta d y \int_{0}^{2 *} d \theta
$$
}




$$
f^{(0)}=\frac{e}{m(m I)^{3 / 2}} \exp \left\{-\left(\frac{m C^{2}}{2 k T}+\frac{I N^{2}}{2 k T}\right)\right\}
$$

Com o conhecimento da funçäo de distribuiçäo $f^{(0)}$ podemos determinar as grandezas mactoscópicas no estado de equilibrio; é o que faremos na próxima seção.

\subsection{Descrição Macroscópica do Estado de Equilíbrio}

Com a lunção de distribuição do squilibrio (2.47) podemos obter grandezas que caractertzam o estado de equilíbrio de um gás poliatônico.

O tensor pressão, definido pela equação $(2.12)$, tem a forma

$$
p_{i j}^{E}=\int m C_{i} C_{j} f^{(0)} d \mathrm{c} d \omega=\frac{1}{3} \delta_{i j} \int m C^{2} f^{(0)} d \mathrm{c} d \omega,
$$

tealizando as integraçōes nas velocidades com a equação (2.47), obtemos

$$
p_{i j}^{E}=\frac{k}{m} T \delta_{i j}=p \delta_{i j}
$$

onde $p=g \frac{k}{m} T$ é pressão hidrostática do găs.

Para o vetor fluxo de calor, definido pela equaçäo (2.16), temos

$$
q_{i}^{E}=\int\left(\frac{1}{2} m C^{2}+\frac{1}{2} I \omega^{2}\right) f^{(0)} d \mathbf{c} d \omega
$$

e realizando as integraçôes, resulta

$$
q_{i}=0
$$

Ým fluido que obdece às equaçoes $(2,49)$ e $(2.51)$, isto é, um fluido nâo viscoso e nảo condutor de calor, é denominado de flutido de Euler.

A entropia especifica do gás para o estado de equilibrio é dada, atravếs da equação (2.20), por

$$
p s^{E}=-k \int f^{(0)} \ln \left(b f^{(0)}\right) d c d \omega
$$

e apôs realizarmos as integrações nas velocidades, obtemos

$$
e^{E}=\frac{k}{m}\left\{\ln \frac{T^{3}}{e}+3-\ln \left[\frac{\left(m l^{3}\right)^{1 / 2}}{(2 \pi k)^{3}} b\right]\right\}
$$

onde $b$ e uma constante arbitrária.

Para ofuxo de entropia em equilibrio, da equaça (2.21), obtemos

$$
\phi_{i}^{E}=-k \int C_{i} f^{(0)} \ln \left(b f^{(0)}\right) d \mathrm{c} d \omega=0 .
$$


Dos princípios de conservação de massa e energia decorre que $f_{1}^{(0)} f^{(0)}=f_{1}^{(0)} f^{(0)} ;$;ssim, da equaçăo (2.31), resulta para a produção de entropia em equilbrio:

$$
\sigma^{E}=0
$$

\subsection{Equações de Balanço para o Estado de Equilíbrio}

A equaçăo de Boltzmann (1.59) para o estado de equilibrio é dada por

$$
\frac{\partial f^{(0)}}{\partial t}+c_{i} \frac{\partial f^{(0)}}{\partial x_{i}}+F_{i} \frac{\partial f^{(0)}}{\partial c_{i}}+\frac{r_{i}}{1} \frac{\partial f^{(0)}}{\partial \omega_{i}}=0 .
$$

Substituindo a funçäo de distribuiçäo (2.47) na equaça anterior e realizando as derivadas, obtemos

$$
\begin{gathered}
f^{(0)}\left\{\frac{1}{\varrho} \frac{\partial \varrho}{\partial t}+\frac{1}{T}\left(\frac{m C^{2}}{2 k T}+\frac{I \Omega^{2}}{2 k T}-3\right) \frac{\partial T}{\partial t}+\frac{m}{k T} C_{i} \frac{\partial v_{i}}{\partial t}\right. \\
\left.+\left(C_{i}+v_{i}\right)\left[\frac{1}{\varrho} \frac{\partial \varrho}{\partial x_{i}}+\frac{1}{T}\left(\frac{m C^{2}}{2 k T}+\frac{I \Omega^{2}}{2 k T}-3\right) \frac{\partial T}{\partial x_{i}}+\frac{m}{k T} C_{j} \frac{\partial v_{i}}{\partial x_{i}}\right]-\frac{m}{k T} C_{i} F_{i}\right\}=0
\end{gathered}
$$

a qual tem a forma polinomial

$$
\alpha^{(\mathfrak{D})}+\alpha_{i}^{(1)} C_{i}+\alpha_{i j}^{(2)} C_{i} C_{j}+\alpha_{i j}^{(3)} C_{i} \Omega_{j} \Omega_{k}+\alpha_{i j}^{(4)} \Omega_{j} \Omega_{j}=0
$$

e válida para todo valor de $C_{i}$ e $\Omega_{i}$; assim, os coeficientes da equação (2.57) são nulos, ou seja

$$
\begin{gathered}
\frac{1}{\rho}\left(\frac{\partial \rho}{\partial t}+v_{i} \frac{\partial \rho}{\partial x_{i}}\right)-\frac{\partial}{T}\left(\frac{\partial T}{\partial t}+v_{i} \frac{\partial T}{\partial x_{i}}\right)=0 \\
\frac{\partial u_{i}}{\partial t}+v_{j} \frac{\partial v_{i}}{\partial x_{i}}+\frac{k T}{m}\left(\frac{1}{\varrho} \frac{\partial \rho}{\partial x_{i}}-\frac{3}{T} \frac{\partial T}{\partial x_{i}}\right)=F_{i} \\
\frac{1}{T}\left(\frac{\partial T}{\partial t}+v_{k} \frac{\partial T}{\partial x_{k}}\right) \delta_{i j}+\left(\frac{\partial v_{i}}{\partial x_{i}}+\frac{\partial v_{j}}{\partial x_{i}}\right)=0 \\
\frac{\partial T}{\partial x_{i}}=0 \\
\frac{\partial T}{\partial t}+v_{i} \frac{\partial T}{\partial x_{i}}=0
\end{gathered}
$$

Defnimos o deviant do tensor gradiente de velocidade como o tensor simétrico e sem traço 


$$
\frac{\partial v_{<i}}{\partial x_{j}}=\frac{1}{2}\left(\frac{\partial v_{i}}{\partial x_{j}}+\frac{\partial v_{j}}{\partial x_{i}}\right)-\frac{1}{3} \frac{\partial v_{k}}{\partial x_{k}} \delta_{i j}
$$

Das equaçoes $(2.61)$ a $(2.63)$ segue que

$$
\frac{\partial v_{i}}{\partial x_{j}}+\frac{\partial v_{i}}{\partial x_{i}}=0
$$

e o traço desta equação é dado por

$$
\frac{\partial u_{k}}{\partial x_{k}}=\nabla \cdot v=0
$$

Assim, das equaçōes (2.64) a (2.66), concluimos que

$$
\frac{\partial v_{\langle i}}{\partial x_{j\rangle}}=0
$$

Se introduzimos a derivada material de uma função arbitrária a( $x, 1)$ por

$$
\dot{a}=\frac{d a}{d t}=\frac{\partial a}{\partial t}+u_{i} \frac{\partial a}{\partial x_{i}}
$$

e, tendo em conta que $p=e \frac{k}{m}{ }^{m}$, podemos escrever as equaçóes $(2.59)$ a $(2.62)$ e $(2.66)$ a $(2.67)$, como

$$
\begin{gathered}
\dot{g}=0 \\
e^{\dot{v}_{i}}=\rho F_{i}-\frac{\partial p}{\partial x_{i}} \\
\dot{T}=0 \\
\frac{\partial T}{\partial x_{i}}=0 \\
\frac{\partial v_{k}}{\partial x_{k}}=0 \\
\frac{\partial v_{<i}}{\partial x_{j>}}=0
\end{gathered}
$$

que săo as equaçôes de balanço para um fiuido em equilibrio sem spin. A equaçäo (2.69) representa a conservaçäo de massa para um fuído incompressivel, condição que explicitada pela equaçăo (2.73). A equaçăo (2.70) è a equação de movimento para um fluido de Euler

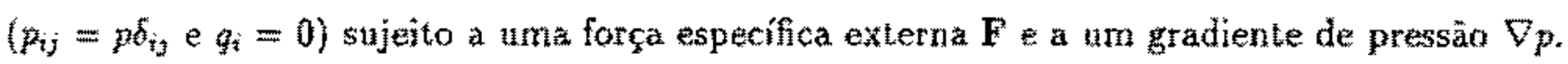
Das equaçōes $(2.71)$ e (2.72) segue que o campo de temperatura $T$ é uniforme estacionário.

Assim, a equaço de Boltzmann (2.56) para um gás poliatômico no estado de equilibrio nos fornece as equaçoes de balanço para um luido de Euler, com as condiçöes adicionais dadas pelas equaçoes $(2.71)$ a $(2.7)$. 


\section{Capítulo 3}

\section{Métodos de Solução da Equação de Boltzmann}

\subsection{Introdução}

Neste capítulo desenvolvemos de maneira sucinta os mátodos clássicos empregados no cálculo dos coeficientes de transporte de gases ideais, os quais são: o método de ChapmanEnskog [9] e o mêtodo de Grad [11]. Estes métodos diferem, fundamentalmente, pelo número de campos básicos empregados.

Recentemente Reinecle e Kremer [43] mostraram que, no calculo das sucessivas aproximaçöes para os coeficientes de transporte de gases ldeais monoatönicos, estes métodos são equivalentes. Com base neste kato, Bezerra Ir., Reinecke e Kremer propuserarn uma forma alternativa para o cálculo dos coeficientes de transporte de gases ideas monoatómicos, denominada método combinado de Chapman-Enskog e Grad [18]. Posteriormente, o método foi empregado para tratar gases ionizados [19]. Também abordaremos este método, com éfáse aos gases ideais poliatốmicos.

No desenvolvimento deste capítulo, consideraremos o gás poliatómico constituido de moléculas modeladas como esferas rígidas, perfeitamente elásticas e rugosas, que a o modelo de Bryan desenvolvido no capitulo 1.

\subsection{O Método de Chapman-Enskog}

A idéla básica deste método consiste em escrever a função de distribuiçăo para o gás. na forma

$$
f(x, c, t, t)=f^{(0)}\left(1+b^{(1)}+b^{(2)}+\cdots\right)
$$

onde $f^{(0)}(x, c, \omega, t)$ é a função de distribuiçäo local de equilibrio, dada pela equaçäo (2.47)

$$
f^{(0)}=\frac{e}{m} \frac{(m I)^{3 / 2}}{(2 \pi k T)^{3}} \exp \left\{-\left(\frac{m C^{2}}{2 k T}+\frac{I T^{2}}{2 k T}\right)\right\}
$$


e nos fornece os valores locais de densidade, velocidade e temperatura; as funçoos $\phi^{(i)}(x, c, \omega, t)$ säo aproximaçöes sucessivas para a funçăo de distribuçâo.

Neste capítulo nos restringiremos à segunda aproximaçấ para a função de distribuição,

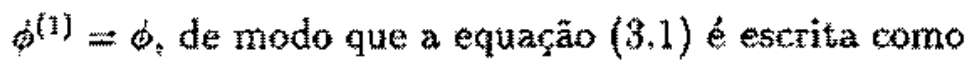

$$
f(x, c, \omega, t)=f^{(0)}(x, c, \omega, t)\lfloor+\phi(x, c, \omega, t)] \text {. }
$$

A solução da equação de Boltzmann para esta aproximaçäo ló obtida, independentemente, por Chapman [7] Enskog [8]. As equacöes constitutivas que dela decorrem conduzern à leis de Navier-Stokes para o tensor pressão e de Fourier para o vetor fluxo de calor, como veremos adiante.

Consideremos um gấs poliatòmico clássico cuja descriçăo é baseada na teoria termodinâmica de cinco campos esealares. Esta teoria tem como objetivo determinar os seguintes campos: densidade de massa $\varrho(x, b)$, velocidade $\ldots(x, l)$ e temperatura $T(x, t)$ ox quais são definidos, através das equaçôes $(2.1),(2.2)$ e $(2.10)$, por

$$
\begin{gathered}
e=\int m \int d c d \omega \\
g w_{i}=\int m c_{i} f d c d \omega^{2} \\
D E=\int\left(\frac{1}{2} m C^{2}+\frac{1}{2} l \Omega^{2}\right) f d c d \omega
\end{gathered}
$$

Para um gás poliatômico ideal constitúdo por moléculas esféricas com energia interna de rotaçào (seis graus de liberdade), a energia específica interna e a temperatura estäo relacionadas auraves da equaçăo $(2.45), \varepsilon=3 \frac{1}{m} T$.

As equaçōes de balanço para estes campos são dadas pelas equaçōes (2.13), (2.14) e (2.17):

$$
\begin{gathered}
\frac{\partial e}{\partial t}+\frac{\partial \varrho v_{i}}{\partial x_{i}}=0 \\
\frac{\partial \eta_{i}}{\partial t}+\frac{\partial}{\partial x_{j}}\left(\rho n_{i} v_{j}+p_{i j}\right)=\underline{Q} F_{i} \\
e \frac{\partial \varepsilon}{\partial t}+2 v_{i} \frac{\partial e}{\partial x_{i}}+\frac{\partial q_{i}}{\partial x_{i}}+p_{i j} \frac{\partial v_{i}}{\partial x_{j}}=0
\end{gathered}
$$

as quais representam, respectivamente, os balanços de massa, densidade de momento linear e energia espectfic à interna.

As equaçöes (3, $\}$ ) a (3.9) näo constituem um sistema de equaç̧es para os campos $\hat{0}, v_{i}$ e $T$ deste que temos dois termos adicionais por ora desconhecidas: o tensor pressäo pi e fluxo 
de calor $q_{i}$. Assim, devemos determinar equaçôs que relacionam o tensor pressäo to buxo de calor com os campos de densidade, velocidade e temperatura. Estas relaçoes dependem do meio a que se referem em termodinânica são conhecidas como equaçós constitutivas.

En leoria cinética a determinação do tensor pressão e do vetor fluxo de calor depende do conhecimento da função de distribuiçăo, de acordo com as definiçoes (2.12) e (2.16), ou seja

$$
\begin{gathered}
p_{i j}=\int m C_{i} C_{j} f d \mathrm{c} d \omega \\
q_{i}=\int\left(\frac{1}{2} m C^{2}+\frac{1}{2} m \Omega^{2}\right) C_{i} f d c d \omega
\end{gathered}
$$

Assim. em seguida, trataremos da determinação do desvio ó para a função de distribuicăo dada pela equaça (3.3), através do nétodo de Chapman-Enskog.

A partir das definiçôes dos campos básicos (3.4) a (3.6) e da equaçăo (3.3), obtemos

$$
\begin{gathered}
Q=\int m f d c d \omega=\int m f^{(0)} d c d \omega \\
g v_{t}=\int m c_{i} f d c d \omega=\int m c_{i} f^{(0)} d c d \omega_{2} \\
T=\frac{m}{3 k \varrho} \int\left(\frac{1}{2} m C^{2}+\frac{1}{2} I \Omega^{2}\right) f d c d \omega=\frac{m}{3 k \varrho} \int\left(\frac{1}{2} m C^{2}+\frac{1}{2} I^{2}\right) f^{(0)} d \mathrm{c} d \omega
\end{gathered}
$$

Das equaçöes $(3.3)$ e (3.12) a (3.14) concluimos que

$$
\int w^{(0)} \phi d \mathrm{c} d \omega=0
$$

onde representa um dos invariantes de somat $m, m c_{i}$ (ou $\left.m C_{i}\right)$ e $\frac{1}{2} m c^{2}+\frac{1}{2} f \omega^{2}$ (ou $\frac{1}{2} m C^{2}+$ $\left.\frac{1}{2} \Omega^{2}\right)$.

Por outro lado, se inserimos a funça de distribuição (3.3) nas definiços (3.10) e (3.11), obtemos:

$$
\begin{gathered}
p_{i j}=\int m C_{i} C_{j} f d \mathrm{c} d \omega=p \delta_{i j}+\int m C_{i} C_{j} f^{(0)} \phi d c d \omega \\
q_{i}=\int\left(\frac{1}{2} m C^{2}+\frac{1}{2} I \Omega^{2}\right) C_{i} \int d c d \omega=\int\left(\frac{1}{2} m C^{2}+\frac{1}{2} I \Omega^{2}\right) C_{i} f^{(0)} \phi d c d \omega
\end{gathered}
$$

As duas equaçoes anteriores inaicam que podernos determinar o tensor prassăo e o vetor fluxo de calor se conhecermos o desvio $\phi$ da funçăo de distribuiça de equilibrio.

No método de Chapman-Enskog, a função de distribuição para um estado fora do equilibrio e deferminada a partir da equaça de Boltzmann. Para o modelo de moleculas como esferas 


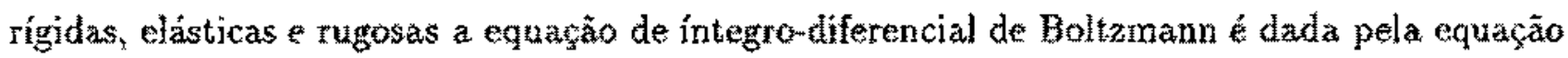
(1.59)

$$
\frac{\partial f}{\partial t}+c_{i} \frac{\partial f}{\partial x_{i}}+F_{\mathrm{i}} \frac{\partial f}{\partial c_{i}}=\int\left(f_{i}^{*} f^{*}-f_{1}\right) a^{2}(\mathrm{~g} \cdot \mathrm{k}) d \mathrm{k} d c_{1} d \omega_{1}
$$

onde nato consideramos torques externos sobre as moleculas $\left(\tau_{i}=0\right)$.

Se substituirmos a equação (3.3) na equaça anterior, obtemos a equação integral

$$
\frac{\partial f^{(0)}}{\partial t}+c_{i} \frac{\partial f^{(0)}}{\partial x_{i}}+F_{i} \frac{\partial f^{(0)}}{\partial c_{i}}=\int f^{(0)} f^{(0)}\left(\phi_{1}^{*}+\phi^{*}-\phi_{1}-\phi\right) a^{2}(\mathrm{~g}, \mathrm{k}) d \mathrm{k} d \mathrm{c}_{1} d \omega_{1}
$$

sendo que: no lado esquerdo desta equaçào mantivemos apenas as derivadas da funçấ $f^{(0)}$, pois săo elas que causarn o desvio $\phi$ na funçà de distribujça $f^{(0)}$; no lado direito, somente 05 termos lineares em $\phi$ foram considerados, pois estamos interessados numa teoria linearizada. Além disso, utilizamos a relacazo $f_{1}^{*(0)} f^{-(0)}=f_{1}^{(0)} f^{(0)}$, que decorre da conservacăo da massa, momento linear e energia numa colisäo binária [veja equaçäo (2.35)].

A equaçăo (3.19) pode ser escrita na forma compacta

$$
D f^{(0)}=I^{*}|\phi|
$$

onde $D$ e $I$ săo operadores definidos por

$$
D \equiv \frac{\partial}{\partial t}+c_{i} \frac{\partial}{\partial x_{i}}+F_{i} \frac{\partial}{\partial c_{i}}
$$

e,

$$
I^{*}[\phi] \equiv \int f_{1}^{(0)} f^{(0)}\left(\phi_{1}^{*}+\phi^{*}-\phi_{1}-\phi\right) a^{2}(\mathrm{~g} \cdot \mathrm{k}) d \mathrm{k} d \mathrm{c}_{1} d \omega_{1}
$$

Ao multiplicarmos a equação $(3.20)$ por uma funçẫo arbitrária $\varphi(x, c, \omega, t)$ e integrarmos nas velocidades e $\omega^{*}$, obtermos

$$
\int \varphi\left(D f^{(0)}\right) d c d \omega=\int \varphi I^{*}[\omega] d \mathrm{c} d \omega
$$

O lado direito da última equaçào pode ser escrito como

$$
\begin{gathered}
\int \varphi I^{*}(\phi) d \mathrm{c} d \omega=\int \varphi f_{1}^{(0)} f^{(0)}\left(\phi_{1}^{*}+\phi^{*}-\phi_{1}-\phi\right) a^{2}(\mathrm{~g} \cdot \mathrm{k}) d \mathrm{k} d \mathrm{c}_{1} d \omega_{1} d \mathrm{c} d \omega= \\
=\int \phi \int_{1}^{(0)} f^{(0)}\left(\varphi_{1}{ }^{*}+\varphi^{\prime}-\varphi_{1}-\varphi\right) a^{2}(\mathrm{~g} \cdot \mathrm{k}) d \mathrm{k} d \mathrm{c}_{1} d \omega_{1} d \mathrm{c} d \omega=\int \phi I[\varphi] d \mathrm{c} d \omega
\end{gathered}
$$

serdo

$$
\mathcal{I}[\phi]=\int f_{1}^{(0)} f^{(0)}\left(\phi_{1}^{\prime}+\phi^{*}-\phi_{k}-\phi\right) a^{2}(\mathrm{~g} \cdot \mathrm{k}) d \mathrm{k} d \mathrm{c}_{1} d w_{1}
$$

A terceifa igualdade da (3.24) é obtida através de argumentos semelhantes aos empregados na dedução do lado direito da equação (1.67). 
Se identificarmos a funçäo arbitrária $\varphi(x, c, w, t)$ com os invariantes de soma $\psi=m, m c_{i} \mathrm{e}$ $\frac{1}{2} m c^{2}+\frac{1}{2} I_{\omega}^{2}$ podemos escrever a equaça $(3.23)$, através da $(3,24), c o m o$

$$
\int \psi\left(D f^{(0)}\right) d c d w=0
$$

que é uma condiçào necessária para que o desvio ḩ seja soluçào da equação integral (3.20).

Aplicando o operador (3.21) à funçăo de distribuição (3.2), obtemos

$$
\begin{gathered}
D f^{(0)}=f^{(0)}\left\{\frac{1}{\rho} \frac{\partial e}{\partial t}+\frac{1}{T}\left(\frac{m C^{2}}{2 k T}+\frac{I \Omega^{2}}{2 k T}-3\right) \frac{\partial T}{\partial t}+\frac{m}{k T} C_{i} \frac{\partial v_{i}}{\partial t}\right. \\
\left.+\left(C_{i}+v_{i}\right)\left[\frac{1}{g} \frac{\partial g}{\partial x_{i}}+\frac{1}{T}\left(\frac{m C^{2}}{2 k T}+\frac{I \Omega^{2}}{2 k T}-3\right) \frac{\partial T}{\partial x_{i}}+\frac{m}{k T} C_{j} \frac{\partial v_{j}}{\partial x_{i}}\right]-\frac{m}{k T} C_{i} F_{i}\right\}
\end{gathered}
$$

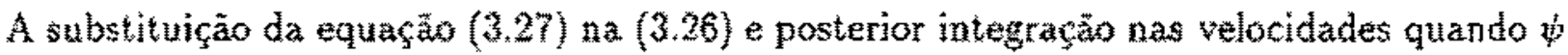
é identificado aos invariantes de soma $m, m c_{i}$ e $\frac{1}{2} m C^{2}+\frac{1}{2} l n^{2}$; resulta nas seguintes equaçós:

$$
\begin{gathered}
\frac{\partial \varrho}{\partial t}+\frac{\partial \vartheta_{i}}{\partial x_{i}}=0 \\
\varrho\left(\frac{\partial v_{i}}{\partial t}+v_{j} \frac{\partial v_{i}}{\partial x_{j}}\right)+\frac{\partial p}{\partial x_{i}}=\varrho F_{i} \\
g \frac{k}{m}\left(\frac{\partial T}{\partial t}+v_{i} \frac{\partial T}{\partial x_{i}}\right)+p \frac{\partial v_{i}}{\partial x_{i}}=0
\end{gathered}
$$

Se compararmos as equacoes (3.7) a (3.9) com (3.28) a (3.30) verificamos que estas últimas sào casos particulares das primeiras. De fato, as equaçoes (3.28) a (3.30) representam equaçoes de balanço para um luido de Euler (fluido năo viscoso e nâo condutor de calor), com $p_{i j}=p t_{i j}$ e $q_{i}=0$.

Se utilizamos as equacöos $(3.28)$ a (3.30) para eliminar as derivadas temporais de (3.27), obtemos

$$
\begin{aligned}
D f^{(0)} & =f^{(0)}\left\{\frac{m}{k T} C_{i} C_{j} \frac{\partial v_{\langle i}}{\partial x_{j}}+\frac{1}{3}\left(\frac{m C^{2}}{2 k T}-\frac{I \Omega^{2}}{2 k T}\right) \frac{\partial v_{*}}{\partial x_{t}}\right. \\
+ & \left.\frac{1}{T}\left[\left(\frac{m C^{2}}{2 k T}-\frac{5}{2}\right)+\left(\frac{I \Omega^{2}}{2 k T}-\frac{3}{2}\right)\right] C_{i} \frac{\partial T}{\partial x_{i}}\right\} .
\end{aligned}
$$

Con base am $(3.22)$ e $(3.31)$ podemos escrever para a equaja $(3.20)$ :

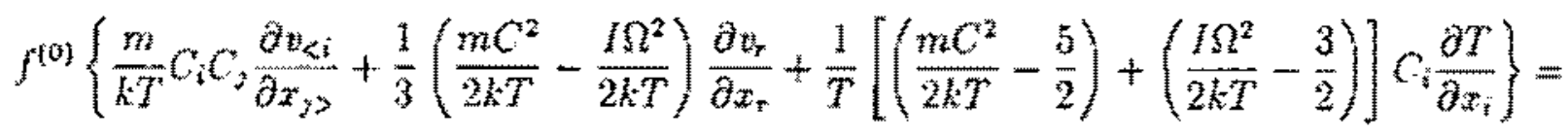




$$
=\int f_{1}^{(0)} f^{(0)}\left(\phi_{1}^{*}+\phi^{*}-\phi_{1}-\phi\right) a^{2}(\mathrm{~g} \cdot \mathrm{k}) d \mathrm{k} d c_{1} d \omega_{1}
$$

que é uma equaçäo integral näo homogênea para o desvio $\phi$. A solução geral para esta equaçăo é dada por

$$
\phi=\phi_{h}+\phi_{p}
$$

onde $\varphi_{h}$ é a soluşão da equação homogênea

$$
\int f_{1}^{(0)} f^{(0)}\left(\phi_{1}^{*}+\phi^{*}-\phi_{1}-\phi\right) a^{2}(\mathrm{~g} \cdot \mathrm{k}) d \mathrm{k} d \mathrm{c}_{1} d \omega_{1}=0
$$

e $\phi_{7}$ e uma soluçắo particular da equaçĩo integral (3.32).

Da equaçäo (3.34) verificamos que a soluçầ $\phi_{\hbar}$ é lal que

$$
\phi_{h i}^{*}+\phi_{h}^{*}=\phi_{h_{1}}+\phi_{h_{h}}
$$

ou seja. $\phi_{h}$ um invariante de soma; assim, podemos escrever que

$$
\phi_{n}=\alpha^{(1)}+\alpha_{i}^{(2)} C_{4}+\alpha_{3}\left(C^{2}+\frac{1}{m} \Omega^{2}\right)
$$

onde $\alpha$ coeficientes escalares $\alpha^{\dot{i}}$ não dependem das velocidades.

Da análise do lado esquerdo da equação (3.32) conclumos qque uma soluçăo particular para essa equaçä̃o é dada por

$$
\begin{gathered}
\phi_{\beta^{p}}=a_{1}\left(\frac{m C^{2}}{2 k T}-\frac{5}{2}\right) C_{i} \frac{\partial T}{\partial x_{i}}+a_{2}\left(\frac{I \Omega^{2}}{2 k T}-\frac{3}{2}\right) C_{i} \frac{\partial T}{\partial x_{i}}+b_{1} C_{i} C_{j} \frac{\partial v_{k i}}{\partial x_{j}} \\
+b_{2}\left(\frac{m C^{2}}{2 k T}-\frac{M^{2}}{2 k T}\right) \frac{\partial \psi_{i}}{\partial x_{t}}
\end{gathered}
$$

onde $a_{1}=a_{1}(\varrho, T), a_{2}=a_{2}(\varrho, T), b_{1}=b_{1}(\varrho, T)$ e $b_{2}=b_{3}(\varrho, T)$ são coeficientes escalares. Assin; a soluçăo geral da equação (3.32) é escrita como

$$
\begin{gathered}
=\omega_{n}+\phi_{p}=a_{1}\left(\frac{m C^{2}}{2 k T}-\frac{5}{2}\right) C_{i} \frac{\partial T}{\partial x_{i}}+a_{2}\left(\frac{I \Omega^{2}}{2 k T}-\frac{3}{2}\right) C_{i} \frac{\partial T}{\partial x_{i}}+b_{1} C_{i} C_{3} \frac{\partial v_{\langle i}}{\partial x_{j\rangle}} \\
+b_{2}\left(\frac{m C^{2}}{2 k T}-\frac{I \Omega^{2}}{2 k T}\right) \frac{\partial v_{i}}{\partial x_{r}}+\alpha^{(n)}+\alpha_{i}^{2} C_{i}+\alpha_{3}\left(C^{2}+\frac{1}{m} n^{2}\right)
\end{gathered}
$$

Da condição dada pela equação (3.15) verificamos que os coeficientes $\alpha^{(0)}, \alpha_{i}^{(2)}$ e $\alpha_{3}$ săo todos nulos: assim, a soluçäo geral para a equaçäo integral (3.32) é escrita como

$$
\phi=a_{1}\left(\frac{m C^{2}}{2 m}-\frac{5}{2}\right) C_{i} \frac{\partial T}{\partial x_{i}}+a_{2}\left(\frac{I N^{2}}{2 m T}-\frac{3}{2}\right) C_{i} \frac{\partial T}{\partial z_{i}}+b_{1} C_{1} C_{j} \frac{\partial v_{\zeta i}}{\left.\partial x_{j}\right\rangle}
$$




$$
+b_{2}\left(\frac{m C^{2}}{2 k T}-\frac{r \Omega^{2}}{2 k T}\right) \frac{\partial v_{r}}{\partial x_{r}}
$$

Com o objetivo de determinar os coeficientes escalates, vamos multiplicar a equaçào (3.32) pela funçäo arbitrária $p(x, c, w, t)$ e integrála nas velocidades, ou seja

$$
\begin{gathered}
\int p f^{(0)}\left\{\frac{m}{k T} C_{3} C_{j} \frac{\partial w_{\zeta}}{\partial x_{j\rangle}}+\frac{1}{3}\left(\frac{m C^{2}}{2 k T}-\frac{I \Omega^{2}}{2 k T}\right) \frac{\partial v_{r}}{\partial x_{r}}+\frac{1}{T}\left[\left(\frac{m C^{2}}{2 k T}-\frac{5}{2}\right)+\right.\right. \\
\left.\left.\left(\frac{I \Omega^{2}}{2 k T}-\frac{3}{2}\right)\right] C_{i} \frac{\partial T}{\partial x_{i}}\right\} d \mathrm{c} d \omega=\int \varphi I[\phi] d \mathrm{c} d \omega=\int \phi I[\varphi] d \mathrm{c} d \omega
\end{gathered}
$$

onde a equaça $(3.24)$ for utilizada.

Se escolhermos a funçă $\psi(x, c, \omega, \bar{l})$, sucessivamente, como

$$
\begin{aligned}
& \varphi=\left(\frac{m C^{2}}{2 k T}-\frac{5}{2}\right) C_{k}, \\
& \psi=\left(\frac{I \Omega^{2}}{2 k T}-\frac{3}{2}\right) C_{k},
\end{aligned}
$$

e realizarmos as integraçóes da equaçäo $(3,40)$ obtemos, respectivamente ${ }^{1}$,

$$
\frac{3}{2} \frac{p}{m} \frac{1}{T} \frac{\partial T}{\partial x_{k}}=\frac{4 \pi}{3}\left(\frac{a \varrho}{m}\right)^{2}\left(\frac{k T}{m}\right)^{3 / 2} \frac{1}{(\kappa+1)^{2}}\left[5 a_{2} \kappa-a_{1}(17 \kappa+4)\right] \frac{\partial T}{\partial x_{k}}
$$

e

$$
\frac{5 p}{2 m} \frac{1}{T} \frac{\partial T}{\partial x_{k}}=\frac{4 \pi}{3}\left(\frac{a g}{m}\right)^{2}\left(\frac{k T}{m}\right)^{3 / 2} \frac{1}{(k+1)^{2}}\left[5 a_{1} k-3 a_{2}\left(2 k^{2}+2 k+1\right)\right] \frac{\partial T}{\partial x_{k}}
$$

Ao resolvermos este sistema de equaçôes para a e $a_{2}$ obtemos

$$
a_{1}=-\frac{45}{8} \frac{m}{a^{2} \varrho T}\left(\frac{m}{\pi k T}\right)^{1 / 2} \frac{(\kappa+1)^{3}(2 \kappa+1)}{\left(102 \kappa^{3}+101 \kappa^{2}+75 \kappa+12\right)}
$$

e

$$
a_{2}=-\frac{3}{2} \frac{m}{a^{2} g T}\left(\frac{m}{\pi k T}\right)^{1 / 2} \frac{(k+1)^{2}(19 k+3)}{\left(102 \kappa^{3}+101 \kappa^{2}+75 \alpha+12\right)}
$$

Para a determinasăo dos coeficientes $b_{1}$ e ba da equaçăo (3.39). consideremos na equạào $(3.40)$ a funçào $\rho(x, c, \omega, t)$, sucessivamente, como

$$
\begin{gathered}
\varphi=C_{\langle r} C_{s\rangle} \\
\psi=\frac{m C^{2}}{2 \cdot T}-\frac{3}{2}
\end{gathered}
$$

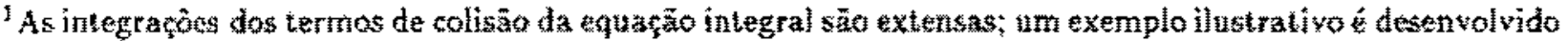
no A pendice :
} 
Inserindo as equaçoes $(3.47)$ e (3.48) na equação (3.40) e realizando as integraçōes nas velocim dades, obtemos

$$
2 \frac{\varrho}{m} \frac{k T}{m} \frac{\partial v_{\langle p}}{\partial x_{s>}}=-\frac{16 \sqrt{\pi}}{15} k_{1}\left(\frac{a g}{m}\right)^{2}\left(\frac{k T}{m}\right)^{3 / 2} \frac{13 n+6}{(k+1)^{2}} \frac{\partial v_{\langle r}}{\partial x_{s>}}
$$

e

$$
\frac{1}{2} \frac{e}{m} \frac{\partial v_{r}}{\partial x_{r}}=-16 \pi b_{2}\left(\frac{a \ell}{m}\right)^{2}\left(\frac{k T}{m}\right)^{1 / 2} \frac{\kappa}{(\kappa+1)^{2}}
$$

on seja,

$$
b_{1}=-\frac{15}{8 g}\left(\frac{m}{a}\right)^{2}\left(\frac{m}{\pi k^{3} T^{3}}\right)^{1 / 2} \frac{(k+1)^{2}}{13 \kappa+6}
$$

e

$$
b_{2}=-\frac{1}{32} \frac{m}{0 a^{2}}\left(\frac{m}{m T^{2}}\right)^{1 / 2} \frac{(\kappa+1)^{2}}{\kappa}
$$

Tendo determinado $a_{1}$ e $a_{2}$ podemos obter a equaça constitutiva para 0 fuxo de calor â Vamos considerar o vetor fluxo de calor (3.11) na lorma

$$
q_{i}=q_{i}^{T}+q_{i}^{R}=\int\left(\frac{1}{2} m C^{2}+\frac{1}{2} r \Omega^{2}\right) C_{i} f^{(0)} \phi d c d \omega
$$

onde ofluxo de calor translacional, relacionado ao transporte de energia cinética transiacional, e definido por

$$
q_{i}^{T}=\int \frac{1}{2} m C^{2} C_{i} f^{(0)} \phi d c d \omega
$$

e o luxo de calor totacional, relacionado ao transporte de energia cinética rotacional, é definido por

$$
d_{i}^{n}=\int \frac{1}{2} \ln ^{2} C_{i} f^{(0)} \phi d d u
$$

Se inseritmos as equaçoes $(3.2)$ e (3.39) na (3.54) e realizamos as integraçoes nas veloci. dades, obtemos para ofluxo de calor translacional

$$
q_{i}^{T}=\frac{5 a_{1}}{2} \varrho\left(\frac{k T}{m}\right)^{2} \frac{\partial T}{\partial x_{i}}
$$

que é a lei de Fourier $\left(q_{i}^{T}=-\lambda^{T} \frac{\partial r}{\partial x_{k}}\right)$; com a equaça (3.45) podemos escrever para o coeficiente de condutividade térmica translacional:

$$
\lambda^{T}=\frac{225}{16 a^{2}}\left(\frac{k^{3} t^{2}}{\pi m}\right)^{1 / 2} \frac{(k+1)^{3}(2 k+1)}{\left(102 k^{3}+101 k^{2}+75 k+12\right)} .
$$

Procedendo de forma análoga com a equaçăo (3.53), obtenos para ofluxo de calor rotacional 


$$
\eta_{i}^{R}=\frac{3 a_{2}}{2} \varphi\left(\frac{k T}{n}\right)^{2} \frac{\partial T}{\partial x_{i}},
$$

com o coeficiente de condutividade térmica rotacional dado, através da equação (3.46), por

$$
\lambda^{R}=\frac{9}{4 a^{2}}\left(\frac{k^{3} T}{\pi m}\right)^{1 / 2} \frac{(\kappa+1)^{2}(19 \kappa+3)}{\left(102 \kappa^{3}+101 \kappa^{2}+75 \kappa+12\right)}
$$

Assim, das equaçoes $(3.57)$ e $(3.59)$, temos para o coeficiente de condutividade térmica do gás

$$
\lambda=\lambda^{F}+\lambda^{T}=\frac{9}{16 a^{2}}\left(\frac{k^{3} T}{\pi m}\right)^{1 / 2} \frac{(\kappa+1)^{2}\left(50 \kappa^{2}+151 \kappa+37\right)}{\left(102 \kappa^{3}+101 \kappa^{2}+75 k+12\right)}
$$

Pura obtermos o tensor pressăo procedemos de maneira semelhante: substituimos as equaçỏes (3.2) e (3.39) na definição (3.16) e, após realizarmos a integraçöes nas volocidades, resulta alei de Navier-Stokes:

$$
p_{i j}=p \delta_{i j}-2 \eta \frac{\partial v_{\langle i}}{\partial x_{j\rangle}}-\eta_{i} \frac{\partial v_{i}}{\partial x_{i}} \delta_{i j}
$$

Com as equaçóes (3.51) e (3.52), temos para os coeficientes da equaçă丶 (3.61):

- coeficiente de viscosidade cisalhante

$$
\eta=e\left(\frac{k T}{m}\right)^{2} b_{1}=\frac{15}{8 a^{2}}\left(\frac{m k T}{\pi}\right)^{1 / 2} \frac{(\kappa+1)^{2}}{(13 x+6)}
$$

- coeficiente de viscosidade volumétrica

$$
\eta_{v}=-e \frac{k T}{m} b_{2}=\frac{1}{32 a^{2}}\left(\frac{m k T}{\pi}\right)^{1 / 2} \frac{(\kappa+1)^{2}}{\kappa}
$$

Com as equaçües $(3.45),(3.46),(3.51)$ e $(3.52)$ podemos escrever o desvio da equação (3.39) como

$$
\begin{aligned}
& \phi=-\frac{2}{5}\left(\frac{m}{k T}\right)^{2}\left[\frac{\lambda^{T}}{\varrho}\left(\frac{m C^{2}}{2 k T}-\frac{5}{2}\right)+\frac{3}{3} \frac{\lambda^{R}}{\varrho}\left(\frac{I \Omega^{2}}{2 k T}-\frac{3}{2}\right)\right] C_{i} \frac{\partial T}{\partial x_{i}} \\
& -\frac{\eta}{\varphi}\left(\frac{m}{k T}\right)^{2} C_{i} C_{j} \frac{\partial v_{\langle i}}{\partial x_{i}}-\frac{\eta_{v}}{\varrho} \frac{m}{k T}\left(\frac{m C^{2}}{2 k T}-\frac{I \Omega^{2}}{2 k T}\right) \frac{\partial v_{z}}{\partial x_{*}}
\end{aligned}
$$

com os coeficientes $\lambda^{T} \cdot \lambda^{A}, \eta$ e $\eta_{y}$ dados, respectivamente pelas equaçöes $(3.57)$ a $(3.59),(3.62)$ e $(3.63)$.

Os resultados descritos pelas equaçoes $(3.60)$ para o coeficjente de condutividade térmira $\lambda$ e (3.62) para o coeficiente de viscosidade cisalhante $\eta$, foram obtidos primeiramente por 
Pidduck [39]. As expressòes para os coeficientes de transporte $\lambda$, $\eta$ e $\eta_{v}$ estâo reproduzidas em Chapman-Cowling [9].

No capitulo 5, empregaremos a terceira aproximaçăo para a função de distribuiçăo $\phi^{(2)}$ na equação (3.1)] na descrição de um gás poliatônico. Obteremos entäo, equações constitutivas que envolvem derivadas de segunda ordem da densidade, velocidade e temperatura. Tais equaçöes sào conhecidas na literatura como equaçoes de Burnett [10].

\subsection{O Método dos Momentos de Grad}

Neste nétodo ${ }^{2}$ o estado macroscópico de um gás poliatômico é descrito através de dezessete campos escalares: densidade de massa $\varrho\left(x_{2}\right)$, densidade de momento linear $\varrho v_{i}\left(x_{*}\right)$, tensor pressấ $p_{i}(x, t)$, temperatura $T(x, t)$, fuxo de calor translacional $q_{i}^{T}(x, t)$, fuxo de calor rotacional $q_{\text {q }}(x, t)$, os quais são definidos por

$$
\begin{gathered}
\varrho=\int m f d c, d \omega, \\
\varrho v_{i}=\int m c_{i} f d c d \omega, \\
p_{i j}=\int m C_{i} C_{j} f d c d \omega, \\
m=\frac{m}{3 k_{\varrho}} \int\left(\frac{1}{2} m C^{2}+\frac{1}{2} I \Omega^{2}\right) \int d c d \omega, \\
q_{i}^{q}=\int \frac{1}{2} m C^{2} C_{i} \int d c d \omega, \\
q_{i}^{n}=\int \frac{1}{2} I \Omega^{2} C_{i} f d c d \omega .
\end{gathered}
$$

As equaçoes de balanço para os dezessete campos escalares são obtidas através das integraçōes da equaçăo de transporte (1.67) ao escolhermos a função arbitráría $\varphi(x, c, \omega, t)$ como:

- $\varphi=m$ (Balanço de Massa)

$$
\frac{\partial \varrho}{\partial t}+\frac{\partial \varrho v_{i}}{\partial x_{i}}=0
$$

\footnotetext{
20 desenvolvimento apresenlado nesta seça
} 
- $\varphi=m c_{i} \quad$ (Balanço de Momento Linear)

$$
\frac{\partial \varrho v_{i}}{\partial t}+\frac{\partial}{\partial x_{j}}\left(\varrho v_{i} z_{j}+p_{i j}\right)=a F_{i}
$$

- $\varphi=m C_{i} C_{j} \quad$ (Balanço para o Tensor Pressäo)

$$
\frac{\partial p_{i j}}{\partial t}+\frac{\partial}{\partial x_{k}}\left(p_{i j k}+p_{i j} v_{k}\right)+p_{k i} \frac{\partial v_{j}}{\partial x_{k}}+p_{k j} \frac{\partial v_{i}}{\partial x_{k}}=p_{i j}
$$

- $p=\frac{1}{3} m C^{2} C_{\mathrm{i}} \quad$ (Balanço do Fluxo de Calor Translacional)

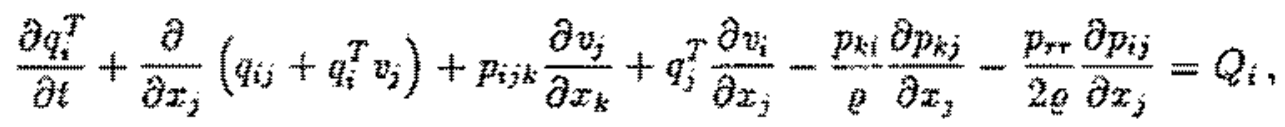

$\varphi=\frac{1}{2} m c^{2}+\frac{1}{2} / \omega^{2} \quad$ (Balanço de Energia)

$$
\frac{\partial}{\partial t}\left(3 \frac{k}{m} \rho T+\frac{1}{2} e v^{2}\right)+\frac{\partial}{\partial x_{i}}\left[q_{i}^{T}+q_{i}^{R}+p_{i j} v_{j}+\left(3 \frac{k}{m} e T+\frac{1}{2} e v^{2}\right) v_{i}\right]=e F_{i} v_{i}
$$

- $\phi=\frac{1}{2} I \Omega^{2} C_{i} \quad$ (Balanço do Fluxo de Calor Rotacional)

$$
\frac{\partial q_{i}^{R}}{\partial t}+\frac{\partial}{\partial x_{j}}\left(h_{i j}+q_{i}^{R} v_{j}\right)+q_{i}^{R} \frac{\partial u_{i}}{\partial x_{j}}-\left(3 \frac{k}{m} \rho T-\frac{1}{2} p_{r+}\right) \frac{\partial p_{i j}}{\partial x_{j}}=H_{i} .
$$

As equatçotes (3.71) a (3.76) não podem ser considerad âs como constituindo um sistema de equaçoes para os campos básico $0, p z_{i}, r, p_{i j}, q_{i}^{T}$ e $q^{R}$ pois contém termos constitutivos que definimos através de:

$$
\begin{aligned}
& p_{j k}=\int m C_{1} C_{j} C_{k} \int d c d w \\
& P_{i}=\int m\left(C_{i}^{*} C_{z}^{*}-C_{i} C_{j}\right) d \Gamma \\
& q_{i j}=\int \frac{1}{2} m C^{2} C_{i} C_{i} f d \mathbf{c} d w \\
& Q_{i}=\int \frac{1}{2} m\left(C^{2} C_{i}^{\prime}-C^{2} C_{i}\right) d \Gamma, \\
& h_{i j}=\int \frac{1}{2} I \Omega^{2} C_{i} C_{j} f d c d \omega \\
& H_{i}=\int \frac{I}{2}\left(\Omega^{22} C_{i}^{\prime}-\Omega^{2} C_{i}\right) d \Gamma
\end{aligned}
$$


onde

$$
d \Gamma=f f_{1} a^{2}(\mathbf{g} \cdot \mathbf{k}) d \mathbf{k} d \mathbf{c}_{1} d w_{1} d \mathbf{c} d \omega
$$

As equaçöes para $p_{i j k} q_{i j}$ e $h_{i j}$ säo denorninadas de momentos da função de distribuição en. quanto que $P_{i j}, Q_{i}$ e $H_{i}$ sắ termos de produção devido as colisòes. Assim, para obtermos um sisterna fechado de equaçồ de campo devemos determinar a funçăo de distribuição $f\left(x_{1}, \omega, t\right)$ en termos dos campos básicos.

Com este objetivo, Kremer [44] utilizou o método dos multiplicadores de Lagrange para a maximizaça da entropia (utilizamos este método no capitulo 4), e obteve a seguinte funçäo de distribuiçăo de Grad para o gás poliatônico com moléculas modeladas como esferas ruggosas:

$$
\begin{aligned}
f=\frac{e}{m} \frac{(m I)^{3 / 2}}{(2 \pi k T)^{3}} \exp \{ & \left.-\left(\frac{m C^{2}}{2 k T}+\frac{I \Omega^{2}}{2 k T}\right)\right]\left\{1+\frac{1}{2 \varrho}\left(\frac{m}{k T}\right)^{2}\left[C_{i} C_{j} p_{k i s}+\left(C^{2}-\frac{I \Omega^{2}}{m}\right) \mathrm{I}\right.\right. \\
& \left.\left.+2\left(\frac{m C^{2}}{5 k T}-1\right) C_{i} q_{i}^{T}+2\left(\frac{I \Omega^{2}}{3 k T}-1\right) C_{i} q_{i}^{R}\right]\right\} .
\end{aligned}
$$

Nesta equaçào introduzimos o deviante do tensor pressầo

$$
p_{<i j\rangle}=p_{i j}-\frac{1}{3} p_{r \tau} \delta_{i j}
$$

e a pressào dinámica $I$ para o gás, definita como a parte fora do equilibrio do traço do tensor pressào, ou seja

$$
\Pi=\frac{1}{3}\left(p_{r r}-p_{r r}^{E}\right)=\frac{1}{3} p_{r r}-\frac{k}{m} T
$$

A pressão dinămica também pode ser escrita em termos da funçăo de distributção f se considerarmos a equaçäo (2.45) para a energía específica por molécula do gás e a equaçăo (3.67) para a definiçăo do tensor pressão: obtemos, entăo

$$
\Pi=\frac{1}{3} \int\left(\frac{1}{2} m C^{2}-\frac{1}{2} m \Omega^{2}\right) f d c d \omega
$$

Inserindo a funça de distribuição (3.84) nas definiçōes dos momentos da funçāo de distribuiçâo (3.77) $(3.79),(3.81)$ e realizando as integraços nas velocidades, resultam:

$$
\begin{aligned}
& p_{i j k}=\frac{2}{5}\left(\delta_{i j} q_{k}^{T}+\delta_{i k} q_{j}^{T}+\delta_{j k} q_{i}^{T}\right) \\
& q_{i j}=\left[\frac{5}{2} m\left(\frac{k T}{m}\right)^{2}+5 \Pi \frac{k T}{m}\right] \hat{b}_{i j}+\frac{7 k T}{2} \frac{k T}{m} p_{\langle i j\rangle} \\
& h_{i, j}=\frac{3}{2}\left(\frac{k T}{m}\right)^{2} \delta_{i j}+\frac{3 k T}{2} \frac{k T}{m}\langle i j\rangle \text {. }
\end{aligned}
$$


Realizando as integraçōes para os termos de produçẫo ${ }^{3}(3.78),(3.80)$ e $(3.82)$, obternos:

$$
\begin{gathered}
P_{i j}=\frac{\rho a^{2}}{m}\left(\frac{\pi k T}{m}\right)^{1 / 2} \frac{1}{(\kappa+1)^{2}}\left[-\frac{32 \kappa}{3} \Pi g_{i j}-\frac{8}{15}(13 \kappa+6) p_{<i j}\right], \\
Q_{i}=\frac{\rho a^{2}}{m}\left(\frac{\pi k T}{m}\right)^{1 / 2} \frac{1}{(\kappa+1)^{2}}\left[-\frac{8}{15}(17 \kappa+4) q_{i}^{T}+\frac{40 \kappa}{9} q_{i}^{R}\right], \\
H_{i}=\frac{\rho a^{2}}{m}\left(\frac{\pi k T}{m}\right)^{1 / 2} \frac{1}{(\kappa+1)^{2}}\left[\frac{8 \kappa}{3} q_{i}^{T}-\frac{8}{3}\left(2 \kappa^{2}+2 \kappa+1\right) q_{i}^{n}\right]
\end{gathered}
$$

Assim, os termos constitutivos estäo determinados em temos dos campos básicos, através das equaçōes $(3.88)$ a $(3.93)$.

Se inserirnos $(3.88)$ a $(3.93)$ nas equaçoes de balanco $(3.71)$ a $(3.76)$, obtemos o seguinte sistema de equaçoes de campo para os dezessete campos escalares básicos:

$$
\begin{aligned}
& \dot{\rho}+\hat{\partial} \frac{\partial p_{i}}{\partial x_{i}}=0 \\
& Q \dot{v}_{i}+\frac{\partial p_{i j}}{\partial x_{j}}=F_{i}
\end{aligned}
$$

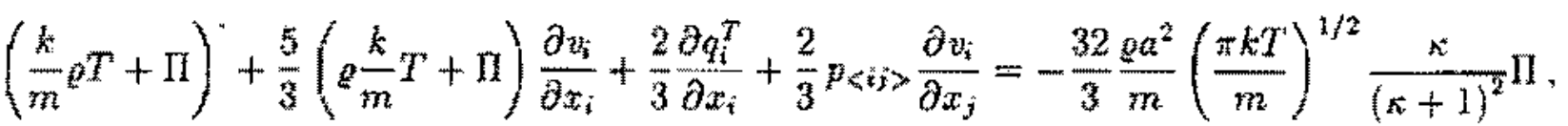

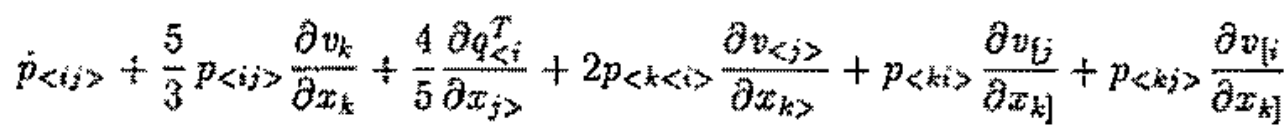

$$
\begin{aligned}
& +2\left(\frac{k}{m} \rho T+\Pi\right) \frac{\partial v_{\langle i}}{\partial x_{j\rangle}}=-\frac{8}{15} \frac{g a^{2}}{m}\left(\frac{\pi k T}{m}\right)^{1 / 2} \frac{(13 k+6)}{(k+1)^{2}} p_{\langle i j\rangle} \\
& a_{i}^{T}+\frac{7}{5} q_{i}^{T} \frac{\partial v_{j}}{\partial x_{i}}+\frac{7}{5} q_{i}^{T} \frac{\partial v_{i}}{\partial x_{j}}+\frac{2}{5} q_{i}^{T} \frac{\partial w_{j}}{\partial x_{i}}+\frac{5}{2}\left(\frac{k T}{m}+\frac{\Pi}{g_{i}}\right) \frac{\partial \Pi}{\partial x_{i}}+\frac{5}{2} \frac{k T}{m}\left(\frac{1}{T} \frac{\partial T}{\partial x_{i}}-\frac{1}{\varrho} \frac{\partial \rho}{\partial x_{i}}\right) \Pi
\end{aligned}
$$

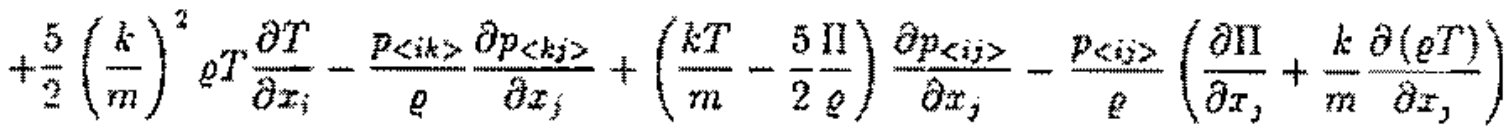

\footnotetext{
No apendice $C$ apresentamos am exemplo para ste tipo de integragăa.
} 


$$
\begin{aligned}
& +\frac{7}{2 m} p_{i j i>} \frac{\partial T}{\partial x_{j}}=\frac{g a^{2}}{m}\left(\frac{\pi k T}{m}\right)^{1 / 2} \frac{1}{(k+1)^{2}}\left[-\frac{8}{15}(17 \kappa+4) q_{i}^{T}+\frac{40 k}{9} q_{i}^{k}\right] \\
& \frac{k}{m} e T+\frac{\partial q_{i}^{k}}{\partial x_{i}}+\frac{\partial q_{i}^{T}}{\partial x_{i}}+\left(\frac{k}{m} \mathrm{e} T+\Pi\right) \frac{\partial \psi_{i}}{\partial x_{i}}+p_{<i j\rangle} \frac{\partial w_{i}}{\partial x_{j}}=0 \\
& q_{i}^{p_{i}}+q_{i}^{R} \frac{\partial v_{k}}{\partial x_{k}}+q_{j}^{R} \frac{\partial v_{i}}{\partial x_{j}}+\frac{3 \Pi}{2} \frac{\partial}{g}\left(\frac{\partial \Pi}{\partial x_{i}}+\frac{\partial p_{<i j}}{\partial x_{j}}+\frac{k}{m} T \frac{\partial \varrho}{\partial x_{i}}+\frac{k}{m} g \frac{\partial T}{\partial x_{i}}\right)+\frac{3}{2} \frac{k}{m}\left(\frac{k}{m} g T \delta_{i j}+p_{<i j\rangle}\right) \frac{\partial T}{\partial x,} \\
& -\frac{3 k}{2} T \frac{\partial \Pi}{\partial x_{i}}=\frac{g a^{2}}{m}\left(\frac{\pi k T}{m}\right)^{1 / 2} \frac{1}{(\kappa+1)^{2}}\left[\frac{8 \kappa}{3} q_{i}^{T}-\frac{8}{3}\left(2 \kappa^{2}+2 \kappa+1\right) q_{i}^{R}\right] .
\end{aligned}
$$

As equaços (3.96) e (3.97) representam, respectivamente, o traço a parte sem traço da equaçu (3.73). Alérn disso, os pontos superescritos representam derivadas materiais definidas pela equação (2,68).

Para obtermos as equaçốes constitutivas para os campos pressão dinânùca $\Pi$, deviante do

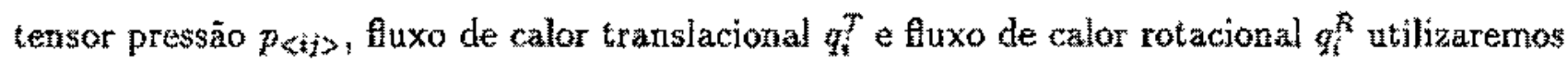
o método de iteraça de Maxwell [28], o qual descreveremos em seguida.

Inicialmente eliminamos $\dot{T}$ da equaçăo $(3.96)$ atravês da equação $(3.99)$; então, obtemos

$$
\Pi+\left(\frac{1}{3} \frac{k}{m} \rho^{T}+\frac{4}{3} \Pi\right) \frac{\partial v_{i}}{\partial x_{i}}+\frac{1}{3} \frac{\partial}{\partial x_{i}}\left(g_{i}^{T}-q_{i}^{R}\right)+\frac{p<\omega_{3}}{3} \frac{\partial v_{i}}{\partial x_{i}}=-\frac{32}{3} \frac{g^{2}}{m}\left(\frac{\pi k T}{m}\right)^{1 / 2} \frac{\kappa}{(\kappa+1)^{2}} \Pi
$$

Em seguida, inserimos os valores de equilibrio

$$
\begin{aligned}
& \Pi^{(0)}=0 \\
& p_{<i j>}^{(0)}=0 \\
& q_{i}^{T(0)}=0 \\
& q_{i}^{n(0)}=0,
\end{aligned}
$$

no lado esquerdo das equaços $(3.97),(3.98),(3.100)$ e $(3.101)$; se negligenciarmos os termos näo lineares obtemos para o lado direito destas equaçoes os valores para a primeira iteração:

$$
2 \frac{k}{m} \rho T \frac{\partial v_{<i}}{\partial x_{j>}}=-\frac{8}{15} \frac{g a^{2}}{m}\left(\frac{\pi k T}{m}\right)^{1 / 2} \frac{(13 \kappa+6)}{(\kappa+1)^{2}} p_{\langle i j\rangle}^{(1)}
$$




$$
\begin{aligned}
& \frac{5}{2}\left(\frac{k}{m}\right)^{2} \rho T \frac{\partial T}{\partial x_{i}}=-\frac{e^{2}}{m}\left(\frac{\pi k T}{m}\right)^{1 / 2} \frac{1}{(\kappa+1)^{2}}\left[\frac{8}{15}(17 \kappa+4) q_{i}^{T^{(1)}}-\frac{40 \kappa}{9} q_{i}^{R(1)}\right] \\
& \frac{3}{2}\left(\frac{k}{m}\right)^{2} \rho T \frac{\partial T}{\partial x_{i}}=\frac{\rho a^{2}}{m}\left(\frac{\pi k T}{m}\right)^{1 / 2} \frac{1}{(k+1)^{2}}\left[\frac{8 \kappa}{3} q_{i}^{T(1)}-\frac{8}{3}\left(2 \kappa^{2}+2 \kappa+1\right) q_{i}^{R(1)}\right] \\
& \frac{1}{3} \frac{k}{m} \rho T \frac{\partial y_{i}}{\partial x_{i}}=-\frac{32}{3} \frac{\rho a^{2}}{m}\left(\frac{m k}{m}\right)^{1 / 2} \frac{\kappa}{(n+1)^{2}} \Pi^{(n)}
\end{aligned}
$$

As equaçoes $(3.106)$ e $(3.109)$ podem ser escritas corro

$$
p_{\langle i j\rangle}^{(1)}=-2 \eta \frac{\partial v_{\langle i}}{\partial x_{j\rangle}}, \quad \Pi^{[1]}=-\eta_{i} \frac{\partial z_{i}}{\partial x_{i}}
$$

que são relações de Navier-Stokes com os coeficientes de transporte dados por:

- coeficiente de viscosidade cisalhante

$$
\eta=\frac{15}{8 a^{2}}\left(\frac{m k T}{n}\right)^{1 / 2} \frac{(\kappa+1)^{2}}{13 k+6}
$$

* coeficiente de viscosidade volumétrica

$$
\eta_{v}=\frac{1}{32 a^{2}}\left(\frac{m k T}{\pi}\right)^{1 / 2} \frac{(k+1)^{2}}{n} .
$$

Para as equaçôes $(3.107)$ e $(3.108)$, temos

$$
q_{i}^{\gamma(1)}=-\lambda^{x^{2}} \frac{\partial T}{\partial x_{i}}, \quad q_{i}^{R^{(1)}}=-\lambda^{R} \frac{\partial T}{\partial x_{i}}
$$

que săo relaçòes de Fourier com os coeficientes de transporte identificados por:

- coeficiente de condutividade térmica translacional

$$
\lambda^{T}=\frac{225}{16 a^{2}}\left(\frac{k^{3} T}{\pi m}\right)^{1 / 2} \frac{(\kappa+1)^{3}(2 \kappa+1)}{\left(102 \kappa^{3}+101 \kappa^{2}+75 k+12\right)}
$$

* coeficiente de condutividade térmica rotacional

$$
\lambda^{R}=\frac{9}{4 a^{2}}\left(\frac{k^{3} T}{7 m}\right)^{1 / 2} \frac{(k+1)^{2}(19 \kappa+3)}{\left(102 k^{3}+101 \kappa^{2}+75 \kappa+12\right)}
$$


Para o vetor fluxo de calor temos, das equaçóes (3.113):

$$
q_{i}^{(1)}=q_{i}^{r(1)}+q_{i}^{R(1)}=-\left(A^{T}+\lambda^{R}\right) \frac{\partial T}{\partial x_{i}}=-\lambda \frac{\partial T}{\partial x_{i}}
$$

com o coeficiente de condutividade térmica dado por

$$
\lambda=\lambda^{T}+\lambda^{R}=\frac{9}{16 a^{2}}\left(\frac{k^{3} T}{\pi m}\right)^{1 / 2} \frac{(\kappa+1)^{2}\left(50 \kappa^{2}+151 \kappa+37\right)}{\left(102 \kappa^{3}+101 \kappa^{2}+75 \kappa+12\right)} .
$$

Os resultados expressos pelas equaçóes $(3.111),(3.112),(3.114),(3.115)$ e (3.117) para os coeficientes de transporte são os mesmos obtidos através do método de Chapman-Enskog desenvolvido na seção 3.2 [veia equaçôes $(3.57),(3.59),(3.60),(3.62)$ e $(3.63)]$.

A densidade e fluxo de entropia podem ser determinadas através das definiçōes (2.20) e $(2,21)$, ou seja

$$
\rho^{s}=-k \int f \ln f d \mathrm{c} d \omega
$$

e

$$
\phi_{i}=-k \int C_{i} f \ln f d c d \omega,
$$

onde consideramos a constante $b=1$. A função de distribuiçầ de Grad (3.84) pade ser escrita na forma

$$
f=f^{(0)}(1+6)
$$

onde $f^{(0)}$ è a funçăo de distribuiçăo do equilibrio,

$$
f^{(0)}=\frac{\varrho}{m} \frac{(m I)^{3 / 2}}{(2 \pi k T)^{3}} \exp \left\{-\left(\frac{m C^{2}}{2 k T}+\frac{1 \Omega^{2}}{2 k T}\right)\right\},
$$

e o desvio do equilíbrio é identiffcado por

$$
0=\frac{1}{2 a^{2}}\left(\frac{m}{m T}\right)^{2}\left[C_{i} C_{j} p_{<i j\rangle}+\left(C^{2}-\frac{m^{2}}{m}\right) \Pi+2\left(\frac{m C^{2}}{5 k T}-1\right) C_{i} q_{i}^{T}+2\left(\frac{M \Omega^{2}}{3 k T}-1\right) C_{i} q_{i}^{R}\right]
$$

Considerando que

$$
\ln (1+x) \simeq x-\frac{x^{2}}{2}, \text { para } x<1
$$

podemos escrever alé a ordem $\phi^{2}$ e com a equação (3.120), que

$$
f \ln l=f^{(0)}\left[(1+\phi) \ln f^{(0)}+\phi+\frac{\phi^{2}}{2}\right]
$$

onde 


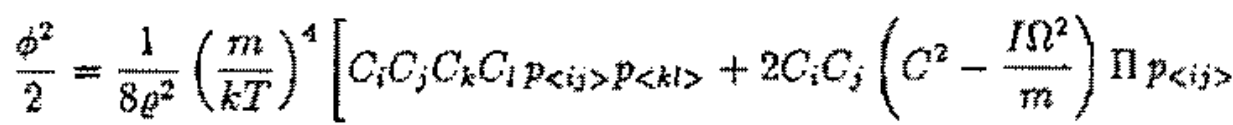

$$
\begin{aligned}
& +4\left(\frac{m C^{2}}{5 k T}-1\right) C_{i} C_{j} C_{k} p_{\langle i j\rangle} q_{k}^{T}+\left(\frac{I \Omega^{2}}{3 k T}-1\right) C_{i} C_{j} C_{k} p_{\langle i j\rangle} q_{k}^{A} \\
& +\left(C^{2}-\frac{I \Omega^{2}}{m}\right)^{2} \Pi^{2}+4\left(\frac{m C^{2}}{5 m}-1\right)\left(C^{2}-\frac{I \Omega^{2}}{m}\right) C_{i} \Pi q_{i}^{T} \\
& +4\left(\frac{M \Omega^{2}}{3 k T}-1\right)\left(C^{2}-\frac{\Pi^{2}}{m}\right) C_{i} \Pi q_{i}^{R}+4\left(\frac{m C^{2}}{5 k T}-1\right)^{2} C_{i} C_{j} q_{i}^{T} q_{j}^{T} \\
& \left.+8\left(\frac{m C^{2}}{5 k T}-1\right)\left(\frac{I \Omega^{2}}{3 k T}-1\right) C_{i} C_{j} q_{i}^{T} q_{j}^{R}+4\left(\frac{I n^{2}}{3 k T}-1\right)^{2} C_{j} C_{j} q_{i}^{n} q_{j}^{n}\right] \text {. }
\end{aligned}
$$

Da equação (3.121) temos

$$
\ln f^{(0)}=\ln \left[\frac{g}{m} \frac{(m I)^{3 / 2}}{(2 \pi k)^{3}}\right]-\left(\frac{m C^{2}}{2 k T}+\frac{I \Omega^{2}}{2 k T}\right)
$$

Inserindo as equagotos (3.124) e (3.126) na (3.118), resulta

$$
\begin{gathered}
p s=-k \ln \left[\frac{\varrho}{m} \frac{(m I)^{3 / 2}}{(2 \pi k T)^{3}}\right] \int f^{(0)}(1+\phi) d c d \omega+k \int f^{(0)}\left(\frac{m C^{2}}{2 k T}+\frac{I n^{2}}{2 k T}\right)(1+\phi) d c d \omega \\
-k \int f^{(0)}\left(\phi+\frac{\phi^{2}}{2}\right) d c d \omega
\end{gathered}
$$

Substituindo as equacōes $(3.121),(3.122)$ e (3.125) na equação anterior e realizando as integraçōes nas velocidades c e $w$ obtemos para a densidade de entropia:

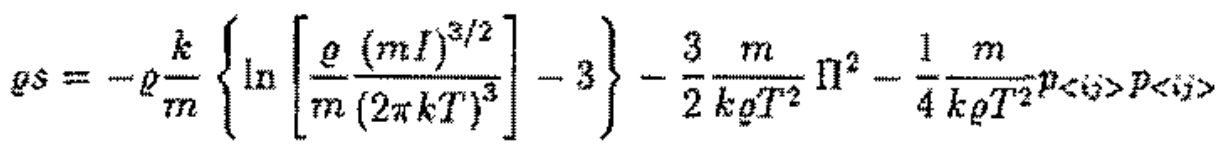

$$
\begin{aligned}
& -\frac{m^{2}}{k^{2} \varrho T^{3}}\left(\frac{1}{5} q_{i}^{T} q_{i}^{T}+\frac{1}{3} q_{j}^{R} q_{j}^{R}\right) .
\end{aligned}
$$

Das equações $(3.119),(3.124)$ e $(3.126)$, temos

$$
\begin{aligned}
& o_{i}=-k \ln \left[\frac{g}{m(2 m l)^{3 / 2}}\right] \int C_{i} f^{(0)}(1+\varphi) d \mathrm{c} d \omega+k \int\left(\frac{m C^{2}}{2 k T}+\frac{I \Omega^{2}}{2 k T}\right) C_{i} f^{(0)}(1+\varphi) d c d \omega \\
& \left.-k \int C_{i} f^{(0)} \phi d c d \omega-k \int C_{i} f^{(0)} \frac{d^{2}}{2} d \mathrm{c} d \omega\right] \text {. }
\end{aligned}
$$

Inserindo as equagoes $(3.121),(3.122)$ e (3.125) na equaçăo anterior realizando as integraçôes nas velocidades, resulta para o fuxo de entropia:

$$
\omega_{i}=\frac{1}{T}\left(a_{i}^{T}+q_{i}^{n}\right)+\frac{m}{k \rho^{T}}\left(q_{i}^{R}+q_{i}^{T}\right) \Pi-\frac{2}{5 k Q^{2}} q_{j}^{T} p_{\langle i j}
$$




\subsection{O Método Combinado de Chapman-Enskog e Grad}

Este método, desenvolvido por Kremer, Reinecke e Bezerra Jr. [18], tem por objetivo a determinação das equaçōes constitutivas para o tensor presš̃a p p para o vetor fluxo de calor gi. Fundamenta-se nos métodos de Chaprnan-Enskog e dos momentos de Grad apresentados, respectivarnente, nas seçōes 3.2 e 3.3 .

As principais vantagens do emprego do método combinado, são:

i) 落o é necessärio obter a soluçăo da equação integral (3.32) do método de Chapman-Enskog: ii) năo é necessário o emprego das equaçồs de campo para os momentos da funçäo de distribuiçäo (3.94) a (3.100) do método dos momentos de Grad.

Em seguida, vamos apresentar os principais pontos no desenvolvimento do método combinado considerando um gás poliatónico constituido de moléculas modeladas como esferas rígidas. perfeitamente elásticas e rugosas. Consideraremos, alén disso, uma teoria de dezessete campos escalares como a desenvolvida na seçäo anterior.

No método combinado a funçăo de distribuiçäo para o gás num estado próximo ao do equilibrio ê a função de dístribuiça de Grad (3.84). Escrevendo- forma da equaça (3.3) do método de Chapman-Enskog, temos:

$$
\begin{aligned}
f=f^{(0)}(1+\phi)=f^{(0)}\{1 & +\frac{1}{2 e}\left(\frac{m}{k T}\right)^{2}\left[C_{i} C_{j} p_{\langle i j\rangle}+\left(C^{2}-\frac{I \Omega^{2}}{m}\right) \Pi+2\left(\frac{m C^{2}}{5 k T}-1\right) C_{i} q_{i}^{T}\right. \\
& \left.\left.+2\left(\frac{M \Omega^{2}}{3 k T}-1\right) C_{i} q_{i}^{R}\right]\right\}
\end{aligned}
$$

onde a funça de distribuiçăo do equillotio é dada pela equação (3.2)

$$
f^{(0)}=\frac{e}{m(m I)^{3 / 2}} \exp \left\{-\left(\frac{m C^{2}}{2 k T}+\frac{1 \Omega^{2}}{2 k T}\right)\right\}
$$

e desvio da funçăo de distribuição do equilíbrio é

$$
\phi=\frac{1}{2 g}\left(\frac{m}{k T}\right)^{2}\left[C_{i} C_{\left.i B_{i}\right\rangle}+\left(C^{2}-\frac{M \Omega^{2}}{m}\right) \Pi+2\left(\frac{m C^{2}}{5 k T}-1\right) C_{i} q_{i}^{T}+2\left(\frac{m^{2}}{3 k T}-1\right) C_{i} q_{i}^{k}\right]
$$

No método de Chapman.Enskog a equação integral näo homogénea para o desvio î dada pela equaçăo (3.32), ou seja

$$
f^{(0)}\left\{\frac{m}{h T} C_{i} C_{j} \frac{\partial v_{\zeta i}}{\partial x_{j}}+\frac{1}{3}\left(\frac{m C^{2}}{2 k T}-\frac{I N^{2}}{2 k T}\right) \frac{\partial v_{r}}{\partial x_{r}}+\frac{1}{T}\left[\left(\frac{m C^{2}}{2 k T}-\frac{5}{2}\right)+\left(\frac{1 \Omega^{2}}{2 k T}-\frac{3}{2}\right)\right] C_{i} \frac{\partial T}{\partial x_{i}}\right\}=
$$




$$
=I^{*}[\phi]=\iint_{1}^{(0)} f^{(0)}\left(\phi_{1}^{*}+\phi^{*}-\phi_{1}-\phi\right) a^{z}(\mathrm{~g} \cdot \mathrm{k}) d \mathrm{k} d \mathrm{c}_{1} d \omega_{1} \text {. }
$$

Inserindo o desvio $\phi$ da (3.133) na equação anterior, resulta:

$$
\begin{aligned}
& f^{(0)}\left\{\frac{m}{k T} C_{k} C_{1} \frac{\partial v_{<k}}{\partial x_{l>}}+\frac{1}{3}\left(\frac{m C^{2}}{2 k T}-\frac{I \Omega^{2}}{2 k T}\right) \frac{\partial v_{k}}{\partial x_{k}}+\frac{1}{T}\left[\left(\frac{m C^{2}}{2 k T}-\frac{5}{2}\right)+\left(\frac{I \Omega^{2}}{2 k T}-\frac{3}{2}\right)\right] C_{k} \frac{\partial T}{\partial x_{k}}\right\}= \\
& =\frac{\left.p_{\langle k}\right\rangle}{2 \varrho}\left(\frac{m}{k T}\right)^{2} \mathcal{L}^{*}\left[C_{k} C_{l}\right]+\frac{\Pi}{2 \varrho}\left(\frac{m}{k T}\right)^{2} \mathcal{T}^{*}\left[C^{2}-\frac{I \Omega^{2}}{m}\right]+\frac{q_{k}^{T}}{T}\left(\frac{m}{k T}\right)^{2} T^{*}\left[\left(\frac{m C^{2}}{5 k T}-1\right) C_{k}\right] \\
& +\frac{q_{k}^{R}}{g}\left(\frac{m}{k T}\right)^{2} T^{*}\left[\left(\frac{I^{2}}{3 k T^{2}}-1\right) C_{k}\right] \text {. }
\end{aligned}
$$

Se multiplicarmos a equaçäo anterior por $\left(C^{2}-\frac{m^{2}}{m}\right)$ e integrarmos a equação resultante nas velocidades, obtemos

$$
\Pi=-\eta_{v} \frac{\partial v_{s}}{\partial x_{k}}
$$

onde

$$
\eta_{z}=-\frac{2}{3} \frac{e^{2}}{m}\left(\frac{k T}{m}\right)^{2}\left\{\int I\left[\frac{m C^{2}}{2 k T}-\frac{I \Omega^{2}}{6 k T}\right]\left(C^{2}-\frac{m n^{2}}{m}\right) d c d \omega\right\}^{-1}
$$

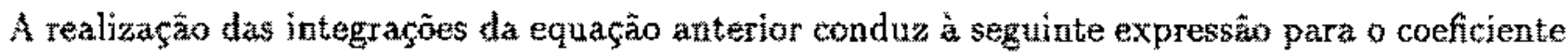
de viscosidade volumètricat

$$
\eta_{v}=\frac{1}{32 a^{2}}\left(\frac{m k T}{\pi}\right)^{1 / 2} \frac{(\kappa+1)^{2}}{\kappa}
$$

De maneira análoga, se multiplicarmos a equação $(3.135)$ por $C_{\longleftrightarrow} C_{j}$ e integramos a equaçäo resultante nas velocidades, obtemos:

$$
p_{\left\langle i Y_{i}\right\rangle}=-2 \eta \frac{\partial v_{<i}}{\partial x_{j\rangle}},
$$

onde

$$
\eta=-10 \frac{\theta^{2}}{m}\left(\frac{k T}{m}\right)^{3}\left\{C_{<k} C_{l} T\left[C_{<k} C_{b}\right] d e d \omega\right\}^{-1}
$$

Realizando as integraçöes da equaçăo anterior obtemos para o coeficiente de viscosidade cisalhante:

$$
\eta=\frac{15}{8 a^{2}}\left(\frac{m k T}{\pi}\right)^{1 / 2} \frac{(\kappa+1)^{2}}{(13 \kappa+6)}
$$

Para a determinarmos o vetor Ruxo de calor multiplicamos a equaça (3.135), sucessiva. mente por $\frac{m C^{*}}{2 k T} C_{t} \in \frac{\Omega^{2}}{2 k T} C_{i}$ integramos as equaçoes resultantes nas velocidades e obternos 0

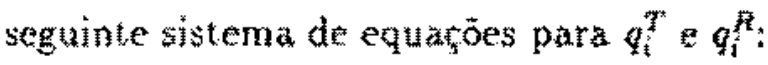




$$
\begin{gathered}
\frac{15}{2}\left(\frac{k^{3 / 2}}{m^{2}} a^{T}\right)^{2} \frac{\partial T}{\partial x_{i}}=q_{i}^{T} \int \frac{m C^{2}}{2 k T} C_{j} I\left[\left(\frac{m C^{2}}{5 k T}-1\right) C_{j}\right] d c d \omega \\
+q_{i}^{n} \int \frac{m C^{2}}{2 k T} C_{j} I\left[\left(\frac{I n^{2}}{3 k T}-1\right) C_{j}\right] d c d \omega
\end{gathered}
$$

e

$$
\begin{gathered}
\frac{9}{2}\left(\frac{k^{3 / 2}}{m^{2}} e T\right)^{2} \frac{\partial T}{\partial x_{i}}=a_{i}^{2} \int \frac{I \Omega^{2}}{2 k T} C_{j} I\left[\left(\frac{m C^{2}}{5 k T}-1\right) C_{j}\right] d \mathrm{c} d \omega \\
+q_{i}^{R} \int \frac{I \Omega^{2}}{2 k T} C_{j} I\left[\left(\frac{I \Omega^{2}}{3 k T}-1\right) C_{j}\right] d \mathrm{c} d \omega .
\end{gathered}
$$

Integrando as duas últimas equaçōes a resolyendo o sistema resultante para $q_{i}^{T}$ e $q_{i}^{R}$, obtemos

$$
q_{i}^{T}=-\lambda^{T} \frac{\partial T}{\partial x_{i}}, \quad q_{i}^{R}=-\lambda^{R} \frac{\partial T}{\partial x_{i}}
$$

onde o coeficiente de condutividade térmica translacional é dado por

$$
\lambda^{T}=\frac{225}{16 a^{2}}\left(\frac{k^{3} T}{\pi m}\right)^{1 / 2} \frac{(k+1)^{3}(2 k+1)}{\left(102 \kappa^{3}+101 \kappa^{2}+7 n k+12\right)}
$$

e o coeficiente de condutividade térmica rotacional é dado por

$$
A^{R}=\frac{9}{4 a^{2}}\left(\frac{k^{3} T}{\pi m}\right)^{1 / 2} \frac{(k+1)^{2}(19 \kappa+3)}{\left(102 \kappa^{3}+101 \kappa^{2}+75 \kappa+12\right)}
$$

Das equaçōes $(3.145)$ e $(3.146)$ resulta para o coeficiente de condutividade térmica:

$$
\lambda=\lambda^{T}+\lambda^{R}=\frac{9}{16 a^{2}}\left(\frac{k^{3} T}{\pi m}\right)^{1 / 2} \frac{(\kappa+1)^{2}\left(50 \kappa^{2}+151 \kappa+37\right)}{\left(102 \kappa^{3}+101 \kappa^{2}+75 \kappa+12\right)} .
$$

As equaçöes $(3.138),(3.141)$ e (3.145) a (3.147) para os coeficientes de tuansporte do gás poliatônico säo as mesmas obtidas anteriormente com os métodos de Chapman-Enskog a dos momentos de Grad.

No capítulo 4 empregaremos o método combinado de Chapman-Enskog e Grad com o objetivo de estudar o comportamento de um gás poliatómico sob a infuência de um campo magnético externo (efeito Senftleben-Beenakker). 


\section{Capítulo 4}

\section{Gases Poliatômicos em Campos Magnéticos Externos}

\subsection{Introdução}

Nosso objetivo neste capitulo é obter os coeficientes de transporte para um gás poliatômico submetido a um campo magnético uniforme estacionário através do método combinado de Chapman-Enskog e Grad, apresentado na seçäo 3.4. Consideremos inicialmente alguns aspectos gerais do problema.

En 1930 foi observado por Senftleben [45] que o gás paramagnatico oxigenio ( $\left.\mathrm{O}_{2}\right)$ apresenta. va pequena diminuição no valor de seu coeficiente de condutividade térmica, quando submétido a um campo magnético uniforme e estacionário. Dois anos após, Engelhardt e Sack [46] verificaram que este efeito também ocorria com o coeficiente de viscosidade cisalhante para o mesmo gass.

Uma explicaçăo qualitativa para o denominado efeito Senfleben foi apresentada por Gorter [47], alguns anos mais tarde. Consideremos, por exemplo, um gảs poliatómico paramagnético composto por moléculas com momento de dipolo magnético permanente $\boldsymbol{t}$. Sob um campo magnético aplicado $\mathbf{H}$ as moléculas do gás interagem com o campo, de modo que o eixo de rothȩăo da molécula precessiona em torno da direção de $\mathbf{H}$ com freqüência de Larmor $\omega_{L}$. Como a seçâo transversal de choque de una molécula nàomesférica depende da sua orientaçäo no espaço, resulta uma seça transversal que varia periodicamente. Desde que os coeficientes de transporte do yás dependem da seçäo de choque da molecula, entäo eles dependerào do campo magnético.

Para uma molécula com momento de inércia $l$ e momento angular $\mathbf{L}=l \omega$, a frequèència de precessão de Larmor é dada por $\omega_{\downarrow}=\frac{2 \pi}{\eta}=\gamma H$, onde $\tau_{L}$ e $\gamma=\mu / L$ são, respectivam mente, o periodo de precessão e a rapảo giromagnética da molécula. O efeito molecular do campo magnético resulta de uma competiçăo entre dois tempos caracteristicos: $\tau_{2}$ e $\tau$, o tempo médio entre duas colisöes moleculares consecutivas. Para $w_{\mathrm{L}} t=2 \pi \frac{t}{r_{2}} \ll 1$ o elpito do campo 
magnético é pequeno pois a seça de choque da molecula muda pouco entre colisóes consecu* tivas, No entanto, para valores tais que $\omega_{\mathrm{L}} \tau>1$ a molécula precessiona várias vezes entre colistzes consecutivas de modo que, tanto a seçăo de choque quanto a orientaçâo da velocidade angular w devem mudar significativamente.

$O$ tempo médio $r$ entre colisoes é inversamente proporcional a densidade $\varrho$ do gás on seja: para uma dada temperatura $T_{y}$ é inversamente proporcional à pressão hidrostática $p=0 \frac{k}{m} T$. Assim, $w_{\mathrm{L}} r$ é proporcional à razăo entre a intensidade $H$ do campo magnético a pressäo $p$ do gás. Portanto, o efeito de um campo magnético aplicado sobre um um gảs poliatômico paramaguético deve depender do parametro $H / p$. A saturação do efeito é alcançada para grandes valores de $H / p$, isto $e_{,}$para $w_{2} T \gg 1$.

Outro efeito da precessäo molecular é que da ocasiona un aumento da seçăo de choque sfetiva transversal da molécula; como os coeficientes de transporte säo inversamente proporcionais aos valores médios calculados sobre as seçôes de choque transversais, resulta uma redução nos valores dos coeficientes de transporte do gás. O efeito observado é pequeno, em torno de $1 \%$. mas mensurátel.

As principais conclusōes sobre estes trabalhos iniciais, sào:

(i) sob campo magnético constante, os coeficientes de condutividade térmica e viscosidade cisalhante de gases paramagnéticos apresentam pequen diminuiçăo, ern torno de $1 \%$ para campos magnéticos de saturação;

(ii) para temperatura constante, os efeitos do campo magnético $H$ dependem somente do pââmetro H/p. onde $p$ é a pressäo do gás;

(ii) o efeito não pode ser explicado em termos de alinhamentos das moléculas com o campo pois, nas condições experimentaís empregadas, a energia potencial magnética 1 H para uma molécula é muito menor que a energia de agitaçấo térmica molecular $k T$.

Estas conclusōes permaneceram praticamente esquecidas até o início da década de 1960 quando Beenakker e seus colaboradores, da Universidade de Leidea, retomaram o estudo do efeito Senftleben. Dentre outras conclutöes, descobriram que o fenómeno ocorre n a só para os gases paramagnéticos como também para os diamagnéticos [48]. Verificaram que, para vários gases, a saturacăo é alcançada para valores wipicos de induços magnéticas da ordem de $1 \mathrm{~T}$ e pressöes da ordem $100 \mathrm{~Pa}$. Na literatura, o efeito passou ser denominado de efeito Senftleben. Beenakker,

Para ilustrar a abordagem do problema do ponto de vista teórico, consideremos as modificajöes que devem ser introduzidas no método de Chapman-Enskog para gases monoatómicos a fim de que possamos estendê-lo aos gases poliatômicos.

Na presenç de forças temodinamicas, como gradientes da temperatura $T$ e da velocidade 
hidrodinâmica $v_{i}$ a funçã uma fungäo maxwelliana isotrópica e passa a depender vetorialmente da velocidade peculiar molecular $G_{i}(\mathrm{x}, t)=c_{i}-v_{i}(\mathrm{x}, t)$. O desvio $\hat{\phi}\left(\mathrm{x}, \mathrm{c}_{y} t\right)$ da funça maxwellianta propocional ăs forças termodinâmicas, e pode ser escrito como $[9]$

$$
\phi=-A_{i} \frac{\partial T}{\partial x_{i}}-B_{<i j>>} \frac{\partial \nu_{<i}}{\partial x_{j\rangle}}
$$

onde $A_{i}$ um vetor e $B_{<i j>}$ é um tensor de segunda ordem, simétrico e sem traço. Se considerarmos que é possivel construir o vetor $A_{i}$ e o tensor $B_{<i j\rangle}$ como funçöes da velocidade peculiar $C_{\text {is }}$ temos:

$$
\phi=-A\left(C^{2}, p_{2}, C_{i} \frac{\partial T}{\partial x_{i}}-B\left(C^{2}, \varrho, T\right) C_{k i} C_{j>} \frac{\partial v_{k i}}{\partial x_{j\rangle}}\right.
$$

onde $A$ e $B$ sato funçoes escalares.

Para un găs constituido de moléculas com graus de liberdade internos de rotaçäo, Kagan 4 Afanase'v [20] mostraram que, além da velocidade translacional $c_{i}$, devemos incluir o pseudovetor velocidade angular rotacional $\omega_{i}$ da molécula, de modo que funçăo de distribuiçăo fora do equilitrio passa a ser escrita como $f(x, c, \omega, t)$. Nesta nova formulacäo, as grandezas

$$
(\mathrm{C} \times \mathbf{\Omega})_{i}=\varepsilon_{\mathrm{i} j k} C_{j} \Omega_{k}, \text { e } \Omega_{<i} \Omega_{j\rangle} C_{k}
$$

podem contribuir para o coeficiente de condutividade térmica, enquanto que os tensores

$$
\Omega_{\langle i} \Omega_{j\rangle}, \quad C_{k i} C_{j\rangle} \Omega_{k} \text { e } C_{<i} C_{j\rangle} \Omega_{<k} \Omega_{i\rangle}
$$

podem contribuit para o coeficientes de viscosidade cisalhante e volumétrica do gás [49]. Estes vetores e tensores, que dependem da orientaçäo da velocidade angular molecular, sâo denominados de pola rizaçocs [50]. O vetor $\varepsilon_{i j k} \bigcap_{j} C_{k}$ efreqüentemente referido como vetor de Waldmann.

Em 1962, Kagan e Maksimov [51] empregaram a polarizaçã̃o $\Omega_{<i} \Omega_{j\rangle} C_{z}$ para calcular 。 tensor condulividade térmica para um gás poliatômico pararnagaético sob um campo magnético externo. tendo obtido resultados que concordavam tom os dados experimentais. Obtiveram expressóes para os coeficientes de condutividade témica perpendicular e paralela em termos de integrais de colisäo, também, mostraram que a razāo entre estes dois coeficientes atinge o valor 3 2 para campos magnéticos de saturaçăo $(H \rightarrow \infty)$. Com o mêtodo utilizado, nă obtiveram efeitos transwersais.

Uma extensäo do trabaho de Kagan e Maksimov foi desenvolvida por Knaap e Beenakker [52]; eles obtiveram expressós para as variaçöes relativas dos coeficientes de condutividade lérmica, utilizando a polarizaçäo $\Omega_{\langle i} n_{j} C_{k}$ e variaçoes relativas para os coeficientes de viscosì dade cisalhante empregando as polarizaços $\Omega_{\langle i} \Omega_{j\rangle} C_{\langle i} C_{j\rangle} \Omega_{<i} \Omega_{j\rangle}$. Com o método empregado obtiveram os efeitos transversais para os coeficientes de transporte mas o coeficiente de viscosidade rolumetica resuliou nulo. 
Sob um campo magnético aplicado um gás poliatômico torna-se anisotrópico, de modo que o vetor lluxo de calor é escrito como

$$
q_{i}=-\lambda_{i} \frac{\partial T}{\partial x_{i}}
$$

onde $\lambda_{i j}$ representa $a$ bensor condutividade termica possul trés componentes independentes. Por exemplo, para o campo magnético coincidente com o éxo a de um sistema de coordenadas cartesianas com origem fixa em algum ponto do gats, $\lambda_{i j}$ é representado por

$$
(\lambda)_{i x}=\left(\begin{array}{ccc}
\lambda_{\| 1} & 0 & 0 \\
0 & \lambda_{t} & \lambda_{t r} \\
0 & -\lambda_{t r} & \lambda_{L}
\end{array}\right)
$$

onde $\lambda_{\mid z}, \lambda_{1}$ e $\lambda_{t r}$ são denominados de coeficiente de condutividade térmica paralelo, perpendicular e transversal, respectivamente.

A comparação dos resultados teóricos com os obtidos experimentalmente mostra que a polarização dominante para a condutividade térmica é $\Omega_{\iota_{i}} \Omega_{j\rangle} C_{k}$ [30], embora outras polarizações, como $\varepsilon_{i j k} \Omega_{j} C_{k}$, possarm apresentar contribuiçōes menores [53].

Da mesma forma o tensor pressấo ê dado por

$$
p_{i j}=\left(p-\eta_{v} \frac{\partial v_{r}}{\partial x_{i}}\right) \delta_{i j}-2 \eta_{\langle i j\rangle\langle k i\rangle} \frac{\partial v_{\langle k}}{\partial x_{l\rangle}},
$$

onde os escalares $p$ no representam, respectivamente, a pressão hidrostática e o coeficiente

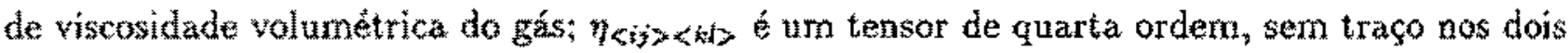
primeiros nos dois ultimos indices e representa os cinco coeficientes escalares independentes de viscosidade cisalhante do gás: $\eta_{1} \eta_{2}$ e $\eta_{3}$ săo coeficientes de viscosidade longitudinais enquanto que al $_{4}$ e n caracterisam os cofficientes de viscosidade transversais. Vários dispositivos expe. rimentais tem sido empregados com objetivo de medir estes coeficientes de viscosidade cisaIhante [54]; a comparaça dos valores calculados com os valores medidos para estes coeficientes indica que a polarizaça dominante neste caso é a polarizaça $\Omega_{<} \Omega_{3}$. Nesta aproximaçăo. tanto o coeficiente de viscosidade volumétrica n quanto o coeficiente de viscosidade cisalhante longitudinal miäs nulos $[50]$.

Para estudar os gases poliatómicos em teoria cinética devemos inicialmente modifcar a rquaçăo de Boltzmann, originalmente obtida para gases monoatomicos rarefeitos. Essa modificaçäo consiste em considerar a funça de distribuição como dependente dos grau de liberdade rotacionais da molécula. ou seja $f=f(\mathrm{x}, \mathrm{c}, \omega, t)$, como comentamos anteriornente. A maior dificuldade no entanto, reside no termo de colisảo da equação de Boltzmann pois a dinămica da colisẫo binária de moléculas poliatómicas não é bem conhecida, mesmo quando consideramos 
somente graus de liberdade internos rotacionais. Além disso, há poucos dados disponiveis sobre as seçôs transversais de choque das moléculas as quais estão diretamente relacionadas com as integrais de colisâo.

Assim, encontramos na literatura duas espécies de abordagem para o problema:

(i) adota-se um modelo molecular para o qual a dinâmica da colisäo binatia ê bem conhecida w, através do método de Chapmana-Enskog com base na equaçăo de Boltzmann modificada, calculam-se os coeficientes de transporte. Neste método resultam, usualmente, um on dois paràmetros livres para serem ajustados aos dados experimentais;

(ii) resolve-se a equaçấo de Boltzmann modificada de uma maneira formal em termos das seçoes transversais de choque, as quais são desconhecidas; obtem-se assim, expressós para os coeficientes de transporte em termos dessas seçoes transversais. A comparaça dos valores destes coeficientes de transporte com os obtidos experimentalmente permite inferir os valores das seçós transversais de choque.

Corn a primeira abordagem, que adotaremos neste trabalho, obtern-se expressoes para os coeficientes de transporte em temos de parämetros moleculares da razão $\mathrm{H} / \mathrm{p}$. Neste esquerna, Klein. Hofman e Dabler [53] utilizaram o modelo de esferas rugosas para as moléculas do gäs, com a faalidade de calcular o tensor condutividade térmica através das polarizaçoes $\varepsilon_{i j k} \Omega_{2} C_{k}$ $\Omega_{<i} \Omega_{j} C_{k}$

Nesta mesma linha, McCourt, Knaap e Moral [55, 56] realizaram cálculos sistemáticos para os coeficientes de condutividade témica (com as polarizaçoes $\Omega_{t_{j}} \Omega_{j>} C_{k}$ e $\Omega_{i} C_{j}$ ), viscosidade cisalhante (com as polarizarônes $\Omega_{\langle i} \Omega_{j\rangle}$ e $\Omega_{i} C_{<j} C_{k>}$ ) e para o coeficiente de viscosidade volumétrica (com a polarizacio $\left.\Omega_{<i} \Omega_{j\rangle} C_{<k} G_{i}\right)$.

Destes trabalhos, verificarnos que o tensor $\Omega_{<i} \Omega_{3} C_{k}$ é a polarizaça dominante na deter-

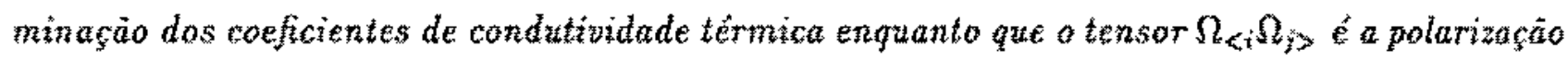

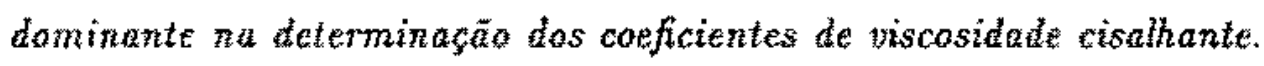

Os resultados numéricos para os coeficientes de transporte que decorrem destes trabalhos näo säo satisfatórios quando comparados com os dados experimentais. Isto se deve principal. mente ao modelo molecular empregado pois para esferas rugosas, mesmo quando as esferas se tocam levernente, os dosvios pós-colisionais säo pronunciados; assim, com o emprego deste modelo esperamos obter apenas resultados qualitativos. No entanto, o modelo empregado fornece estimativas para os coeficientes de transporte bem como é capaz de prever as carateristicas fundamentais do fenomeno, como: (a) a dependéncia dos coeficientes de transporte com o paràmetro $H / p$ : (b) os valores de saturação alcançados pelos coeficientes de transporte longitudinais para grandes valores do parâmetro $H / p$ e (c) os valores mátimos alcançados pelos coeficentes de transporte transversais.

No método combinado de Chapman-Enskog e Crad (veja seçăo 3.4) utilizamos caracteris- 
ticas de ambos os métodos ou seja, empregamos uma equaçäo análoga à equaçäo integral do método de Chapman-Enskog enquanto que o desvio da funçào de distribuiçăo é escrito em termos dos momentos da funçăo de distribuiç̧̃o, como no método dos momentos de Grad. As equaçoes constitutivas para o tensor pressão e vetor fluxo de calor säo obtidas diretamente da equação de Boltzmann através do método de Chapman-Enskog.

As principais vantagens do método combinado cousistem em nu necessitamos resolver a equaçäo integral do método de Chapman-Enskog nem empregar as equaçöes de campo que ocorrem no método dos momentos de Grad. Com isso, conseguimos uma consideráyel redução no processo de cálculo algébrico para a obtençấo dos coeficientes de transporte. Este método fot utilizado para calcular os coeficientes de tranporte de gases monototomicos e suas misturas [18] bem como de gases ionirados [19], com excelentes resultados.

Nosso objetivo neste capitulo ebter os coeficientes de transporte to efeito SenftlebenBeenakker através do método combinado de Chapman-Enskog e Grad. Para isso, consideraremos as moléculas que constituem o gás poliatômico como esferas perfeitamente rigidas, elásticas e rugosas, qué é o modelo de Bryan discutido na seçäo 1.3.

Na descrição do estado macroscópico do gảs empregamos quarenta campos básícos escalares dentre os quais escolhemos campos que estäo relacionados com as seguíntes polarizaçóes; 0 vetor de Wadmann $\varepsilon_{i j k} \Omega_{k} C_{j,}$ o tensor $\Omega_{<j} \Omega_{j\rangle} C_{k}$ que são dominantes na determinaça dos coeficientes de condutividade térmica o tensor $\Omega_{<i} \Omega_{j}$, que é dominante na determinaçäo dos coeftcientes de viscosidade cisalhante; com esta úlima escolha não é possivel obter a dependência do coeficiente de viscosidade volumétrica $\eta_{v}$ com o campo magnético [49].

Para a determinação da função de distribuição que representa o estado do gás próximo do estado de equilibrio em termos dos campos básicos, ou seja, a funçüo de distribuição de Grad para o problema, uthlizamos um método que consiste na maximizaçăo da entropia tendo como vínculos os quarenta campos básicos $[44,57]$.

$\mathrm{Na}$ obtençăo da soluçăo para os coeficientes de condutividade térmica é necessário representar o tensor $h_{\langle i j\rangle}$ : quue decorre da polarizaçäo $\Omega_{<j} \Omega_{j\rangle} C_{k}$, em termos do campo magnético e do vetor fluxo de calor; isto é leito através de teoremas de representação [21], os quais nos fornecem una maneira sistemática para os cálculos dos coeficientes de condutividade têrmica se viermos a introduzir novas polarizaço na teoria.

Das expressöes obtidas para os coeficientes de transporte resultam, como casos particulares, os resultados obtidos por Pidduck [39], para campo magnético nulo, e os resultados de Dabler e colaboradores [53] quando empregamos somente a polarizaçâo $\Omega_{<i} \Omega_{\zeta\rangle} C_{b}$ para o cálculo dos coeficientes de condutividade térmica. 


\subsection{Campos Básicos}

Caracterizaremos o estado macroscópico de um gảs poliatónico através de quarenta campos escalares, os quais süo definidos por:

- densidade de massa:

$$
g(x, b)=\int m f d c d w
$$

- densidade de momento linear:

$$
e v(x, t)=\int m c f d c d \omega
$$

- temperatura:

$$
T(x, t)=\frac{m}{3 k \varrho} \int\left(\frac{1}{2} m C^{2}+\frac{1}{2} I \Omega^{2}\right) \int d \mathrm{c} d \omega,
$$

- Aluxo de calor translacional:

$$
q_{i}^{T}(\mathrm{x}, t)=\int \frac{1}{2} m C^{2} C_{i} f d \mathrm{c} d \omega,
$$

- fuxo de calor rotacional:

$$
q_{i}^{R}(\mathrm{x}, t)=\int \frac{1}{2} I \Omega^{2} C_{i} f d \mathrm{c} d \omega
$$

- deviante do tensor pressão:

$$
p_{<i j>}(x, t)=\int m C_{<i} C_{j>} \int d c d \omega,
$$

- pressab dínámica.

$$
\Pi(x, t)=\frac{1}{3} \int\left(\frac{1}{2} m C^{2}-\frac{1}{2} I \Omega^{2}\right) \int d c d \omega
$$

- $\{<$ in $\rangle(x, t)$.

$$
q_{\langle i j\rangle}(x, b)=\int \ln _{<\left\{a_{j}\right\rangle} \int d c d w
$$

- $h_{i}(x, i)$

$$
h_{i}(x, i)=\int \varepsilon_{i j h} \Omega_{j} C_{k} \int d c d w
$$

- $h_{<i j>t}\left(x_{1} t\right)$

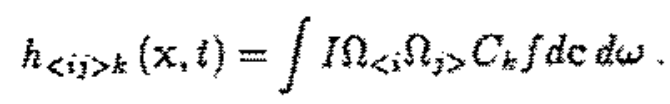

Os campos descritos pelas equaçòes $(4.15)$ a $(4.17)$ näo lem nomes especificos, mas eles săo

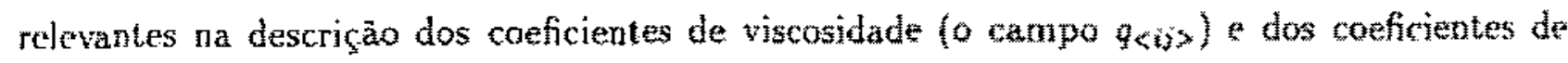
condutividade térmica (os campos $h_{i}$ e $\left.h_{<i j>k}\right)$, como será mostrado adiante. 


\subsection{A Função de Distribuição de Grad}

A determinação da função de distribuição para o gás poliatômico pata um exatado próximo ao estado de equilibrio, será feita através da maximizaçäo da densidade de entropia es [5T] sujeita aos vínculos descritos pelas equaçoes (4.8) a (4.17).

A densidade de entropia, definida pela equação (2.20), édada por

$$
p_{s}=-k \int f \ln f d c d w^{*}
$$

onde tomamos a constuante arbitrária $b=1$.

De acordo com o cálculo variacional este problema é equivalente to da maximizaçầo sem restriçōes. da funcĩo [58]

$$
\begin{aligned}
& F=-\int\left[\ln f+A m+A_{i}^{y} m C_{i}+\lambda\left(\frac{1}{2} m C^{2}+\frac{1}{2} \Omega^{2}\right)+\lambda_{i}^{F} \frac{m C^{2}}{2} C_{i}+\lambda_{i}^{R} \frac{m \Omega^{2}}{2} C_{i}\right. \\
& +A_{i j} m C_{<i} C_{j\rangle}+\lambda^{\prime}\left(\frac{1}{2} m C^{2}-\frac{1}{2} I \Omega^{2}\right)+\lambda_{i j} I \Omega_{<i} \Omega_{j\rangle}+\Lambda_{i} \frac{1}{m} \varepsilon_{i j} \Omega_{j} C_{k} \\
& \left.+\Lambda_{i j k} I \Omega_{\langle i} \Omega_{j\rangle} C_{k}\right] f d \mathrm{c} d \omega
\end{aligned}
$$

onde $A, \Lambda_{i}^{y}, \lambda, \lambda_{i}^{T}, \lambda_{i}, \Lambda_{i j}, \lambda^{*}, \lambda_{i j}, \Lambda_{i}$ e $\Lambda_{i j k}$ são multiplicadores de Lagrange a serem detarminados. Neste caso, a equação de Euler-Lagrange

$$
\frac{\partial F}{\partial f}-\frac{d \partial F}{d t} \frac{\partial F}{\partial f}=0
$$

se reduz a $\frac{\partial F}{\partial f}=0$ e obtemos da equação $(4.19)$ :

$$
\begin{aligned}
& f=\exp \left\{-\left(\frac{m}{k}\right)\left[\frac{k}{m}+\Lambda+\lambda\left(\frac{C^{2}}{2}+\frac{I \Omega^{2}}{2 m}\right)\right]\right\} \exp \left\{( - \frac { m } { k } ) \left[\Lambda_{i}^{y} C_{i}+\lambda_{i}^{T} \frac{C^{2}}{2} C_{i}\right.\right. \\
& +\lambda_{i}^{R} \frac{I}{2 m} \Omega^{2} C_{i}+\lambda_{i j} C_{\langle i} C_{j\rangle}+\lambda^{\prime}\left(\frac{C^{2}}{2}-\frac{I \Omega^{2}}{2 m}\right)+\lambda_{i j} \frac{I}{m} \Omega_{\langle i} \Omega_{j\rangle}+A_{i} \frac{I}{m} \varepsilon_{j j k} \Omega_{j} C_{k} \\
& \left.\left.+\Lambda_{i j k} \frac{I}{m} \Omega_{<i} \Omega_{j>} C_{k}\right]\right\} \text {. }
\end{aligned}
$$

Como estamos interessados em processos próximos do equilibrio, nosso objetivo é obter um função de distribuiçăo na forma $f=f^{(0)}(1+6)$, onde $f^{(0)}$ é a função de distribuiçăo de equilíbrio e ó seu desvio. Consideraremos portanto, a segunda exponencial da equaçäo (4.21) como um termo pequeno que pode ser expandido através de $e^{-x} \approx 1-x$, vảlida para $x \ll 1$. Identificamos o multiplicador $\lambda$ com o inverso da temperatura absoluta $T$ a fim de obtermos a funçào de distribuiçào maxwelliana. Assim, da equaçâo (4.21), obtemos: 


$$
\begin{gathered}
f=\exp \left\{-\left[1+\frac{m}{k} \Lambda+\left(\frac{m C^{2}}{2 k T}+\frac{I \Omega^{2}}{2 k T}\right)\right]\right\}\left\{1-\frac{m}{k}\left[\Lambda_{i}^{y} C_{i}+\lambda_{i}^{T} \frac{C^{2}}{2} C_{i}+\lambda_{i}^{k} \frac{I}{2 m} \Omega^{2} C_{i}\right.\right. \\
+\Lambda_{i j} C_{<i} C_{i>}+\lambda^{y}\left(\frac{C^{2}}{2}-\frac{I \Omega^{2}}{2 m}\right)+\lambda_{i j} \frac{I}{m} \Omega_{<i} \Omega_{j>}+\Lambda_{i} \frac{I}{m} \epsilon_{i j k} \Omega_{j} C_{k} \\
\left.\left.+\Lambda_{i j k} \frac{I}{m} \Omega_{<i} \Omega_{j>} C_{k}\right]\right\} .
\end{gathered}
$$

Para obtermos os multiplicadores restantes, inserimos a equaçõo $(4,22)$ nas defniçóes dos campo: básicos $(4.8)$ a (4.17) $e_{3}$ após as respectivas integraçöes, obtemos:

$$
\begin{aligned}
& A=-\frac{k}{m}\left\{1+\ln \left[\frac{e(m I)^{3 / 2}}{m} \frac{(2 \pi k)^{3}}{(m)}\right.\right. \\
& \Lambda_{i}^{v}+\frac{5 k T}{2} \lambda_{i}^{T}+\frac{3 k T}{2} \frac{k T}{m} \lambda_{i}^{*}=0 \text {, } \\
& \Lambda_{i}^{*}+\frac{7 k T}{2} \frac{\lambda_{i}^{T}}{m}+\frac{3 k T}{2} \frac{k}{m} \lambda_{i}^{R}=-\frac{2 k}{5 m \varphi}\left(\frac{m}{k T}\right)^{2} q_{i}^{T} \\
& \Lambda_{i}^{*}+\frac{5}{2} \frac{k T}{m} \lambda_{i}^{T}+\frac{5}{2} \frac{k T}{m} \lambda_{i}^{R}=-\frac{2 k}{3 m g}\left(\frac{m}{k T}\right)^{2} q_{i}^{R} \\
& \Lambda_{\langle i j\rangle}=-\frac{k}{2 m \varphi}\left(\frac{m}{k T}\right)^{q} p_{\langle i j\rangle} \\
& \lambda^{\prime}=-\frac{k}{m \rho}\left(\frac{m}{k T}\right)^{2} \Pi \\
& \left.\lambda_{\langle i j\rangle}=-\frac{k}{2 m g}\left(\frac{m}{k T}\right)^{2} q\langle i\rangle\right\rangle \\
& A_{i}=-\frac{k}{2 l g}\left(\frac{m}{k T}\right)^{2} h_{i} \\
& \Lambda_{<i j>k}=\Lambda_{i j k}=-\frac{k}{2 m \ell}\left(\frac{m}{k T}\right)^{3} h_{<j j>k} .
\end{aligned}
$$

Do sistema de equaçôes $(4.24)$ a (4.26) resultam

$$
\Lambda_{i}^{*}=\frac{k}{m \varphi}\left(\frac{m}{h T}\right)^{2}\left(g_{i}^{T}+q_{i}^{A}\right)
$$




$$
\begin{aligned}
& \lambda_{i}^{T}=-\frac{2 k}{5 m p}\left(\frac{m}{k T}\right)^{3} \eta_{i}^{T}, \\
& \lambda_{i}^{R}=-\frac{2 k}{3 m p}\left(\frac{m}{k T}\right)^{3} q_{i}^{R} .
\end{aligned}
$$

Assim, as equaçöes (4.23) e (4.27) a (4.34) fornecem os multiplicadores de Lagrange para a equacâo (4.22) em termos dos campos basicos definidos pelas equaçôes (4.8) a (4.17). Substituindo as na equaçào (4.22), obtemos

$$
\begin{aligned}
& f=\frac{\varrho}{m} \frac{(m I)^{3 / 2}}{(2 \pi k T)^{3}} \exp \left\{-\left(\frac{m C^{2}}{2 k T}+\frac{I \Omega^{2}}{2 k T}\right)\right\}\left\{1+\frac{1}{2 \varrho}\left(\frac{m}{k T}\right)^{2} p_{\langle i j\rangle} C_{i} C_{j}\right.
\end{aligned}
$$

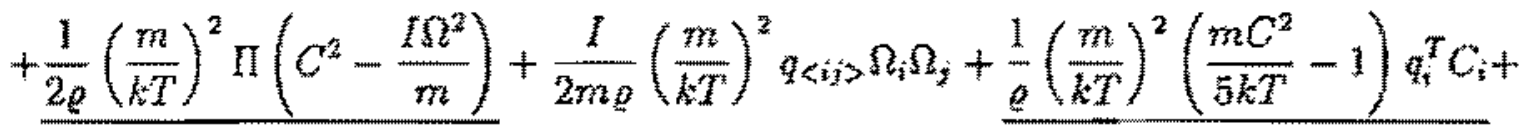

$$
\begin{aligned}
& \left.+\frac{1}{\varrho}\left(\frac{m}{k T}\right)^{2}\left(\frac{I \Omega^{2}}{3 k T}-1\right) q_{i}^{R} C_{i}+\frac{1}{2 \varrho}\left(\frac{m}{k T}\right)^{2} h_{i} \varepsilon_{i j k} \Omega_{j} C_{k}+\frac{I}{2 m \varrho}\left(\frac{m}{k T}\right)^{3} h_{<i j>k} \Omega_{i} \Omega_{i} C_{k}\right\},
\end{aligned}
$$

que é função de distribuição de Grad para o problema. Os termos sublinhados identificam

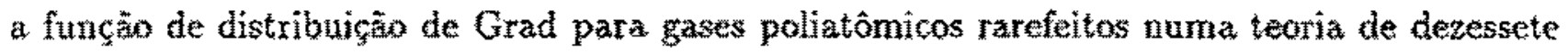
campos escalares, dada pela equação (3.84).

\subsection{O Método Combinado de Chapman-Enskog e Grad}

Consideremos um go poliatôrico sujeto a um campo magnético externo estacionário e uniforme H. O torque magnético $\tau$ sobre uma molécula do gks com momento de inércia $/$ e momento magnético $\mu$, é dado por

$$
r=\frac{d \mathbf{L}}{d t}=\mu \times \mathbf{H}
$$

O momento magnético $\mu$ é proporcional ao momento anguar $\mathrm{L}=1 \Omega$ de modo que

$$
\mu=\gamma I \Omega
$$

onde $\gamma=2 \pi g \mu_{n} /$ h é a razà giromagnética para a molécula, $g$ é o fator rotacional de Landé. f $_{\mathrm{s}}$ o magneton nuclem $e$ h a constante de Planck.

Das equacous $(4.36)$ e $(4.37)$, temos:

$$
\tau=\uparrow(\Omega \times \mathbf{H})
$$


de modo que a equaçăo de Boltamann (1.59) pode ser escrita como

$$
\frac{\partial f}{\partial t}+c_{i} \frac{\partial f}{\partial x_{i}}+\gamma(\mathbf{\Omega} \times \mathbf{H})_{i} \frac{\partial f}{\partial \Omega_{i}}=\int\left(f_{1}^{\prime \prime} f^{*}-f_{1} f\right) a^{2}(\mathrm{~g} \cdot \mathrm{k}) d \mathrm{k} k \mathrm{c}_{1} d w_{1}
$$

onde não consideramos forças externas $\left(F_{1}=0\right)$.

Vamos obter a soluçäo da equação (4.39) através do método combinado de Chapman- Ennskog e Crad. desenvolvido na seção 3.3 .

A funçăo de distribuição de Grad (4.35) pode ser escrita como

$$
f=f^{(0)}(1+\phi)
$$

onde

$$
f^{(0)}=\frac{e}{m} \frac{(m l)^{3 / 2}}{(2 \pi k T)^{3}} \exp \left\{-\left(\frac{m C^{2}}{2 k T}+\frac{m n^{2}}{2 k T}\right)\right\}
$$

é a função de distribuiçăo para o equilibrio,

$$
\begin{aligned}
& \phi=\frac{1}{2 \theta}\left(\frac{m}{k T}\right)^{2} p_{\langle i j\rangle} C_{i} C_{j}+\frac{1}{2 \theta}\left(\frac{m}{k T}\right)^{2} \Pi\left(C^{2}-\frac{m \Omega^{2}}{m}\right)+\frac{I}{2 m \theta}\left(\frac{m}{k T}\right)^{2} q\left\langle i j>\Omega_{i} \Omega_{j}\right. \\
& +\frac{1}{g}\left(\frac{m}{k T}\right)^{2}\left(\frac{m C^{2}}{5 k T}-1\right) q_{i}^{T} C_{i}+\frac{1}{p}\left(\frac{m}{k T}\right)^{2}\left(\frac{I \Omega^{2}}{3 k T}-1\right) q_{i}^{n} C_{i}+\frac{1}{2 p}\left(\frac{m}{k T}\right)^{2} h_{i} \varepsilon_{i j k} \Omega_{j} C_{k} \\
& +\frac{I}{2 m \underline{e}}\left(\frac{m}{k T}\right)^{3} h_{\langle i j\rangle k} \Omega_{i} \Omega_{j} C_{k}
\end{aligned}
$$

é o desvio de $f^{(0)}$.

Se substituirmos a equação (4.40) na (4.39), mantivermos as derivadas de f(0) no primeiro membro somente termos lineares em ho segundo membro, pois estamos interessados numa teoria linearizada, obtemos a seguinte equação:

$$
\frac{\partial f^{(0)}}{\partial t}+c_{i} \frac{\partial f^{(0)}}{\partial x_{i}}+\gamma(\Omega \times \mathbf{H}]_{i} \frac{\partial \phi}{\partial \Omega_{i}}=I^{m}[\phi]
$$

onde introduzimos a notação da equaçäo (3.22)

$$
I^{*}[\phi]=\int f_{1}^{(0)} f^{(0)}\left(\phi_{1}^{*}+\phi^{*}-\phi_{1}-\phi\right) a^{2}(\mathrm{~g} \cdot \mathrm{k}) d \mathrm{k} d c_{1} d \omega_{1} .
$$

Na equaça (4.43) mantivemos o termo proporcional a $\frac{\partial 3}{3 n_{1}}$ por duas razöes: a primeira é que este termo fornece o acoplamento entre campo magnético es campos escalares definidos pelas

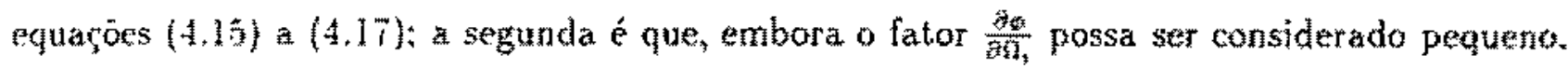
para campos magnéliros suficientemente intensos ele năo é negligenciävel. 
Sendo $y\left(x_{1}, \omega, t\right)$ uma funçăo arbitrátia, a equação $(4,44)$ satisfaz à propriedade dada através da equaciano $(3.24)$, por:

$$
\int \varphi T^{*}[\phi] d \mathrm{c} d \omega=\int \phi \mathcal{L}[\varphi] d \mathrm{c} d \omega
$$

ande

$$
I[\phi]=\int f_{1}^{(0)} f^{(0)}\left(\phi_{1}^{z}+\phi^{\prime}-\phi_{1}-\phi\right) a^{2}(g \cdot \mathrm{k}) d \mathrm{k} d \mathrm{c}_{1} d \mathrm{u}_{1}
$$

Ao multiplicarmos a equafão (4.43) por $\varphi(x, c, w i)$, integrarmos a equaça resultante nas velocidades $c$ e com a equąăo $(4.45)$, obtemos

$$
\int \varphi\left[\frac{\partial f^{(0)}}{\partial t}+c_{i} \frac{\partial f^{(0)}}{\partial x_{i}}+\gamma(\Omega \times \mathbf{H})_{i} \frac{\partial \phi}{\partial \Omega_{i}}\right] d c w^{*}=\int \phi I[\varphi] d c d \omega
$$

Substituindo as equaçōes (4.41) e (4.42) no primeiro membro da equação anterior, resulta:

$$
\begin{aligned}
& \int \varphi f^{(0)}\left\{\frac{1}{\varrho} \frac{\partial \vartheta}{\partial t}+\frac{1}{T}\left(\frac{m C^{2}}{2 k T}+\frac{m n^{2}}{2 k T}-3\right) \frac{\partial T}{\partial t}+\frac{m}{k T} C_{i} \frac{\partial v_{i}}{\partial t}\right. \\
& +\left(C_{i}+v_{i}\right)\left[\frac{1}{\partial} \frac{\partial \varrho}{\partial x_{i}}+\frac{1}{T}\left(\frac{m C^{2}}{2 k T}+\frac{I \Omega^{2}}{2 k T}-3\right) \frac{\partial T}{\partial x_{i}}+\frac{m}{k T} C_{j} \frac{\partial v_{j}}{\partial x_{i}}\right] \\
& \left.+\gamma\left[\frac{I}{m} \frac{q_{\langle p s\rangle}}{\varrho}\left(\frac{m}{k T}\right)^{2} \Omega_{s}+\frac{h_{s}}{2 \varrho}\left(\frac{m}{k T}\right)^{2} \varepsilon_{s p k} C_{k}+\frac{I}{m} \frac{h_{\langle p s>k}}{e}\left(\frac{m}{k T}\right)^{3} \Omega_{s} C_{k}\right](\Omega \times \mathbf{H})_{p}\right\} d \mathbf{c} d \omega= \\
& \left.=\int t \mid \varphi\right] d c d \omega .
\end{aligned}
$$

Se identificarmos a funçäo arbitrária $\varphi(x, c, \omega, t)$ sucessivamente com os invariantes de soma $m$, mo e $\frac{1}{2} m C^{2}+\frac{1}{2} / \Omega^{2}$ e integrarmos as equaços resultantes nas velocidades, obtemos:

$$
\begin{gathered}
\frac{\partial \varrho}{\partial t}+v_{i} \frac{\partial g}{\partial x_{i}}+e \frac{\partial v_{i}}{\partial x_{i}}=0 \\
\varrho\left(\frac{\partial v_{i}}{\partial t}+v_{i} \frac{\partial v_{i}}{\partial x_{j}}\right)+\frac{\partial p_{i}}{\partial x_{i}}=0 \\
3 \frac{k}{m}\left(\frac{\partial T}{\partial t}+v_{i} \frac{\partial T}{\partial z_{i}}\right)+p \frac{\partial v_{i}}{\partial x_{i}}=0
\end{gathered}
$$

que săo equaçóes de campo para um flutido de Euler $\left(p_{z j}=p \delta_{i j}\right.$ e $q_{i}=0$ ). Ao empregramos estas eçuaçóes para eliminar as derivadas temporais da equaçäo (4.48), obtemos

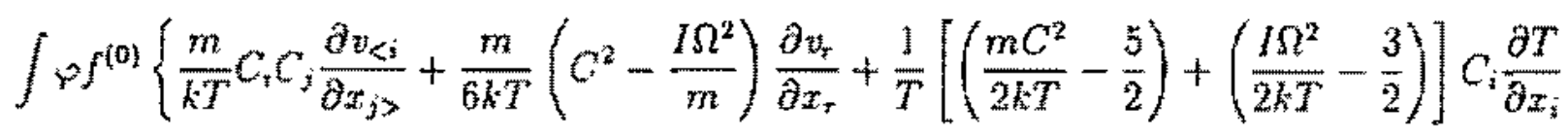




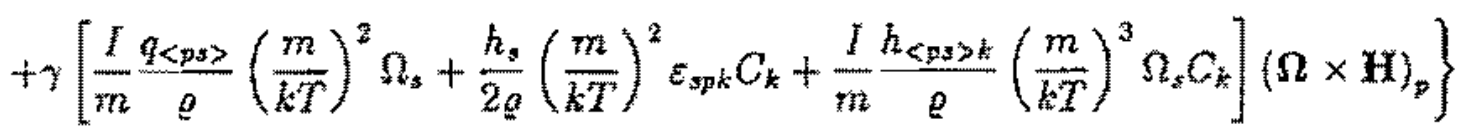

$$
\begin{aligned}
& =\int d I[\phi] d \mathrm{c} d \omega
\end{aligned}
$$

Nas seçöes seguintes trataremos de resolver a eqquaçào (4.52) através de escolhas adequadas da função arbitrária $t(x, c, \omega, t)$. Destas soluçōes decorrerem expressōes para os coeficientes de tramsporte para o gás poliatômieo sob a influência de um campo magnético externo $H$.

\subsection{Coeficientes de Condutividade Térmica}

Ao identificamos a funçăo arbitrária $\varphi(x, c, \omega, t)$ na equaça $(4.52)$, respectivamente, com $\frac{I \Omega^{2}}{2} C_{i}, \frac{m C^{2}}{2} C_{i}, I \varepsilon_{i j} \Omega_{j} C_{k,} I \Omega_{<j} \Omega_{j\rangle} C_{k}$, e integrarmos cada uma das equaçoes obtidas nas velocidades ce $\omega$, obtemos:

$$
\begin{aligned}
& 3 \frac{\varrho}{I}\left(\frac{k T}{m}\right)^{2} \frac{1}{T} \frac{\partial T}{\partial x_{i}}=A_{i}=\int \Omega^{2} C_{i} I[\phi] d \mathrm{c} d w \\
& 5 \frac{b}{m}\left(\frac{k T}{m}\right)^{2} \frac{1}{T} \frac{\partial T}{\partial x_{i}}=B_{i}=\int C^{2} C_{i} I[\phi] d c d w \\
& -\frac{y}{2 I} \varepsilon_{i s m} h_{s} H_{m}=D_{i}=\int \varepsilon_{i j k} \Omega_{j} C_{k} I[\phi] d c d \omega \\
& \frac{\gamma}{I}\left(\varepsilon_{m p r} h_{\langle p i>k} H_{*}+\varepsilon_{p i r} h_{\langle p i\rangle k} H_{r}\right)=E_{<i j\rangle k}=\int \Omega_{\langle i\rangle} \Omega_{j\rangle} C_{k} I[\phi] d \mathrm{~d} d \omega .
\end{aligned}
$$

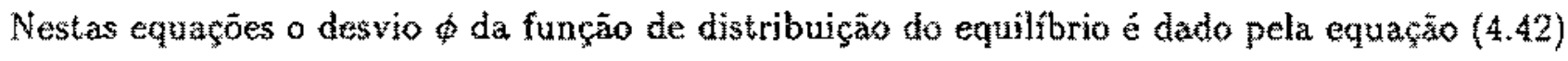
enquanto que o operador $I[\phi]$ é definido pela equaçăo (4.46).

As integraçôs dos termos de colisăo das equaçöes (4.53) a (4.56) requerem cálculos longos e repetitivos; no apêndice $\mathrm{C}$ realizamos, como um exemplo, a integraçẵo de urna integral de colisżo thpica.

As integraçōes das equaçòes $(4.53)$ a $(4.56)$ resultam no seguinte sistema de equaçôs:

$$
\begin{aligned}
& 3 \frac{e}{l}\left(\frac{k T}{m}\right)^{2} \frac{1}{T} \frac{\partial T}{\partial x_{i}}=\frac{32 \sqrt{m}}{3} \frac{e}{m^{2}}\left(\frac{k T}{m}\right)^{1 / 2} \frac{1}{k(\kappa+1)^{2}}\left[\frac{\sqrt{\pi}}{a}(\kappa+1)\left(\frac{k T}{m}\right)^{1 / 2} h_{i}\right. \\
& \left.+\frac{3}{5}(2 \kappa+1) h_{<i t s}-2 \kappa q_{i}^{T}+2\left(2 k^{2}+2 \kappa+1\right) q_{i}^{R}\right] \text {, } \\
& 5 \frac{e}{m}\left(\frac{m T}{m}\right)^{2} \frac{1}{T} \frac{\partial T}{\partial x_{i}}=-\frac{8 \sqrt{\pi}}{3} \frac{g^{2}}{m^{2}}\left(\frac{k T}{m}\right)^{1 / 2} \frac{1}{(n+1)^{2}}\left[k h_{<i l>l}+\frac{2}{3}(17 n+4) q_{i}^{T}-\frac{10}{3} k q_{k}^{k}\right]
\end{aligned}
$$




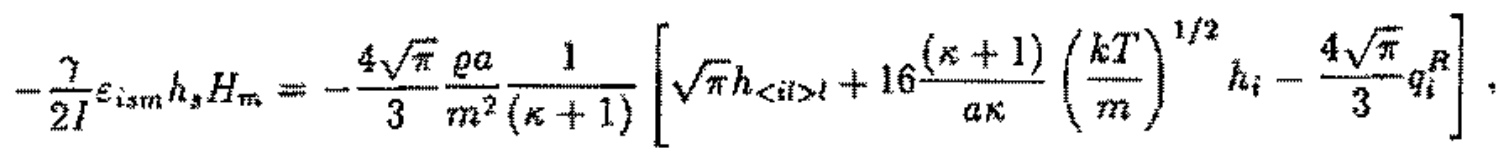

$$
\begin{aligned}
& \frac{\gamma}{I}\left(\varepsilon_{q j} H_{,} h_{<j i>k}+\varepsilon_{\text {mir }} H_{r} h_{<p j>k}\right)=\frac{8 \sqrt{\pi}}{15} \frac{g}{m^{2}}\left(\frac{k T}{m}\right)^{1 / 2} \frac{1}{\kappa(\kappa+1)^{2}}\left\{\frac{5 \sqrt{\pi}}{a}\left(\frac{k T}{m}\right)^{1 / 2}(\kappa+1)\right. \\
& \times\left(\delta_{i k} h_{j}+\delta_{j k} h_{i}-\frac{2}{3} \delta_{i j} h_{k}\right)+4(2 \kappa+1) \delta_{i j} h_{\langle k l>l}-(11 \kappa+3)\left(\delta_{i k} h_{<j l>1}+\delta_{j k} h_{<i}+>l\right) \\
& -2\left(20 \kappa^{2}+16 k+11\right) \hbar_{\langle i j\rangle k}-(\kappa+3)\left(h_{\langle i k>j}+h_{\langle j k>i}\right)-4\left[\kappa\left(\delta_{i k} q_{j}^{T}+\delta_{j k} q_{i}^{T}-\frac{2}{3} \delta_{i j} q_{k}^{T}\right)\right. \\
& \left.\left.+(2 \kappa+1)\left(\delta_{i k} q_{j}^{R}+\delta_{j k} q_{i}^{R}-\frac{2}{3} \xi_{j} q_{k}^{R}\right)\right]\right\}
\end{aligned}
$$

Vamos introduzir as seguintes grandezas reduzidas:

$$
\begin{gathered}
H_{i}^{w}=\frac{\sqrt{\pi} a}{k} h_{i}, \quad H_{<i j\rangle}=\frac{\sqrt{\pi} a^{2} T}{m}\left(\frac{k T}{m}\right)^{3 / 2} h_{<i j\rangle k} \\
Q_{i}^{T}=\frac{\sqrt{\pi} a^{2} T}{m}\left(\frac{m}{k T}\right)^{3 / 2} q_{i}^{T}, \quad Q_{i}^{n}=\frac{\sqrt{\pi} a^{2} T}{m}\left(\frac{m}{k T}\right)^{3 / 2} q_{i}^{n} \\
H_{i}=\frac{\gamma m}{g a^{2}}\left(\frac{m}{\pi k T}\right)^{1 / 2} H_{i}
\end{gathered}
$$

sendo que $H_{i}, H_{<i j\rangle k}, Q_{i}^{T}$ e $Q_{i}^{R}$ tem dimensós de temperatura/comprimento enquanto que $H_{i}$ é adimensional.

Desta forma, o sistema de equaçöes $(4.57) \cdot(4.60)$ pode ser escrito como:

$$
\begin{aligned}
& 3(2 \kappa+1) H_{k} H_{>1}-10 k Q_{i}^{2}+10\left(2 \kappa^{2}+2 \kappa+1\right) Q_{i}^{R}+5 \sqrt{T}(x+1) H_{i}^{w}=-\frac{45}{8}(x+1)^{2} \frac{\partial T}{\partial x_{i}} \\
& 15 k H_{<I I>I}+6(17 \kappa+4) Q_{i}^{T}-50 \kappa Q_{i}^{R}=-\frac{225}{8}(\kappa+1)^{2} \frac{\partial T}{\partial x_{i}}
\end{aligned}
$$

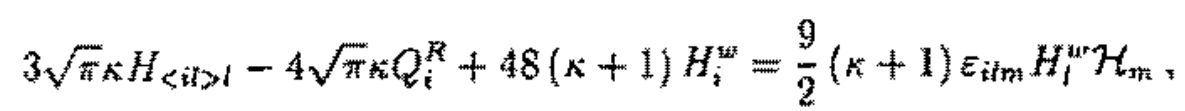




$$
\begin{aligned}
& 5 \sqrt{\pi}(\kappa+1)\left(\delta_{i k} H_{j}^{w}+\delta_{j k} H_{i}^{w}-\frac{2}{3} \delta_{i j} H_{k}^{w}\right)+4(2 \kappa+1) \delta_{i j} H_{<k>l} \\
& -(11 n+3)\left(\delta_{i k} H_{\langle j i>1}+\delta_{j k} H_{\langle k\rangle}\right)-2\left(20 \kappa^{2}+16 x+11\right) H_{\langle i j\rangle k}-(\kappa+3)\left(H_{<i k>j}+H_{\langle j k>i}\right) \\
& -4 \kappa\left(\delta_{i k} Q_{j}^{T}+\delta_{j k} Q_{i}^{T}-\frac{2}{3} \delta_{i j} Q_{k}^{T}\right)-4(2 \kappa+1)\left(\delta_{i k} Q_{j}^{R}+\delta_{j k} Q_{i}^{R}-\frac{2}{3} \delta_{i j} Q_{k}^{R}\right)
\end{aligned}
$$

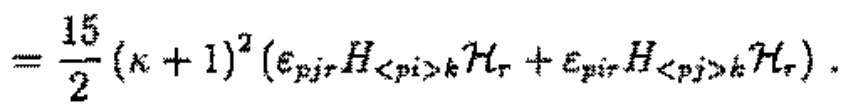

A solução deste sistema de equaçoses para a condutividade térmica reduzida

$$
Q_{i}(\mathcal{H})=Q_{i}^{T}(\mathcal{H})+Q_{i}^{F}(\mathcal{H})
$$

nos permitirá o cálculo do coefciente de condutividade térmica adimensional $\lambda_{i j}(\mathcal{H})$ através da lei de Fourier generalizada

$$
Q_{i}(H)=-\lambda_{i j}(H) \frac{\partial T}{\partial x_{j}}
$$

com o tensor condutividade térnica dado por

$$
\lambda_{i j}^{t}(H)=\frac{1}{a^{2}}\left(\frac{k^{3} T}{\pi m}\right)^{t / 2} \lambda_{i j}(h)
$$

Na resoluçato do sistema de equaçoes $(4.64)$ a (4.67) vamos analisar separadamente a influência de cada um dos campos: $H_{i}^{\text {w }}(\mathbf{x}, t)$, também denominado vetor de Waldmann [50], e do tensor $H_{\langle i j>k}(\mathrm{x}, t)$ sobre o tensor condutividade térmica $\lambda_{i j}$. Consideremos inicialmente, a soluçăo mais simples em que ambos, $H_{i}^{w}$ e $H_{<\text {iłsk }}$, säo nulos.

\subsubsection{A Soluçāo de Pidduck para o Coeficiente de Condutividade Térmica}

Se considerarmos somente os campos $Q_{i}^{T}(x, t)$ e $Q^{R}(x, t)$, o sistema de equaçoes (4.64) a (4.67) se reduz a

$$
\begin{gathered}
-10 \kappa Q_{i}^{T}+10\left(2 \kappa^{2}+2 \kappa+1\right) Q_{i}^{F}=-\frac{45}{8}(\kappa+1)^{2} \frac{\partial T}{\partial x_{i}} \\
6(17 \kappa+4) Q_{i}^{T}-50 \kappa Q_{i}^{R}=-\frac{225}{8}(\kappa+1)^{2} \frac{\partial T}{\partial x_{i}}
\end{gathered}
$$

com soluçōes 


$$
\begin{aligned}
& Q_{i}^{T}=-\frac{225}{16} \frac{(\kappa+1)^{2}(2 \kappa+1)}{\left(102 \kappa^{3}+101 \kappa^{2}+75 \kappa+12\right)} \frac{\partial T}{\partial x_{i}} \\
& Q_{i}^{R}=-\frac{9}{4\left(102 \kappa^{3}+101 \kappa^{2}+75 \kappa+12\right)} \frac{(\kappa+1)^{2}(19 \kappa+3)}{\partial x_{i}}
\end{aligned}
$$

Portanto,

$$
Q_{i}=Q_{i}^{T}+Q_{i}^{R}=-\frac{9}{16} \frac{(\kappa+1)^{2}\left(50 \kappa^{2}+151 \kappa+37\right)}{\left(102 \kappa^{3}+101 \kappa^{2}+75 \kappa+12\right)} \frac{\partial T}{\partial x_{i}}
$$

De acordo com as equaçốs $(4.69),(4.73)$ e (4.74) o coeficjente de condutividade térmita trandiacional é dado por

$$
\lambda_{0}^{T}=\frac{225}{16} \frac{(\kappa+1)^{2}(2 \alpha+1)}{\left(102 \kappa^{3}+101 \kappa^{2}+75 \kappa+12\right)}
$$

e o coeficiente de condulividade térmica rotacional por

$$
\lambda_{0}^{R}=\frac{9}{4} \frac{(\kappa+1)^{2}(19 \kappa+3)}{\left(102 \kappa^{3}+101 \kappa^{2}+75 \kappa+12\right)}
$$

Assim, através da equaçào (4.70), o coeficiente de condutividade térmica do gás $\lambda_{0}^{f}=\lambda_{0}^{\prime T}+\lambda_{0}^{\prime F}$ è dado por

$$
\lambda_{0}^{\prime}=\frac{9}{16} \frac{1}{a^{2}}\left(\frac{k^{3} T}{\pi m}\right)^{1 / 2} \frac{(\kappa+1)^{2}\left(50 \kappa^{2}+151 \kappa+37\right)}{\left(102 \kappa^{3}+101 \kappa^{2}+75 \kappa+12\right)}
$$

que é resultado obtido anteriormente por Pidduck [39].

Notemos que, corr esta escolha para os campos, o coeficiente de condutividade térmica é isotrópico e independente do campo magnético aplicado $H$. Este fato confirma a previsảo de Waldmann [59] de que pelo menos uma polarizaçäo em $\mathbf{\Omega}$ é necessária a firn de que o coeficiente de condutividade de um gás poliatômico apresente ura dependência com o campo magnético aplicado.

Na próxima seçẫo analisaremos a influéncia do campo $H_{i}^{w}(x, t)$ sobre o coeficiente de condutivjdade térmica do gás.

\subsubsection{Campo $H_{i}^{w}(\mathbf{x}, t)$ e Coeficientes de Condutividade Térmica}

Vamos analisar a influência da polarizaç̃o $\epsilon_{i j k} \Omega_{j} C_{k}$ sobre os coeficlentes de condutividade térmica, atraves do campo básico $H_{i}^{w}(\mathrm{x}, \mathrm{t})$ o qual é definido segundo as equaçoes $(4,16)$ e 
$(4.61)$, por

$$
H_{i}^{*}(\mathrm{x}, t)=\frac{\sqrt{\pi} a}{k} \int l \varepsilon_{\mathrm{i} j k} \Omega_{j} C_{k} \int d \mathrm{c} d t
$$

Para os campos $Q_{i}^{T}(\mathrm{x}, t), Q_{i}^{R}(\mathrm{x}, t)$ e $H_{i}^{w}(\mathrm{x}, t)$ o sistema de equaçós $(4.64)$ a (4.67) é escrito como

$$
\begin{gathered}
a_{1} H_{i}^{t}+a_{2} Q_{i}^{T}+a_{3} Q_{i}^{R}=a_{4} \frac{\partial T}{\partial x_{i}} \\
b_{1} Q_{i}^{R}+b_{2} Q_{i}^{R}=b_{3} \frac{\partial T}{\partial \tau_{i}} \\
c_{1} H_{i}^{w}+c_{2} Q_{i}^{R}=c_{3} \varepsilon_{i m} H_{i}^{w} H_{m}
\end{gathered}
$$

onde:

$$
\begin{gathered}
a_{1}=5 \sqrt{*}(k+1), \quad a_{2}=-10 \kappa, \quad a_{3}=10\left(2 \kappa^{2}+2 k+1\right), \quad a_{4}=-\frac{45}{8}(\kappa+1)^{2}, \\
b_{1}=6(17 \kappa+4), \quad b_{2}=-50 \kappa, \quad b_{3}=-\frac{225}{8}(k+1)^{2}, \\
a_{1}=48(k+1), c_{2}=-4 \sqrt{\pi} \kappa, c_{3}=\frac{9}{2}(k+1) .
\end{gathered}
$$

Das equaçóes $(4.80)$ e $(4.81)$ obtemos para ofluxo de calor rotacional reduzido:

$$
Q_{i}^{R}=\frac{\left(a_{4} b_{1}-a_{2} b_{3}\right)}{\left(a_{3} b_{1}-a_{2} b_{2}\right)} \frac{\partial T}{\partial x_{i}}-\frac{a_{1} b_{1}}{\left(a_{3} b_{1}-a_{2} b_{2}\right)} H_{i}^{\text {ts }}
$$

de modo que a equacăo $(4.82)$ pode ser escrita como

$$
\left(\alpha_{1} \delta_{i t}+\varepsilon_{i m} \mathcal{H}_{m}\right) H_{i}^{w}=\alpha_{i} \frac{\partial T}{\partial x_{i}}
$$

onde introduzimos

$$
\begin{gathered}
\alpha_{1}=\frac{a_{1} b_{1} c_{2}}{c_{3}\left(a_{3} b_{1}-a_{2} b_{2}\right)}-\frac{c_{1}}{c_{3}} \\
\alpha_{2}=\frac{c_{2}\left(a_{4} b_{1}-a_{2} b_{3}\right)}{a_{3}\left(a_{3} b_{1}-a_{2} b_{2}\right)} .
\end{gathered}
$$

Para obtermos $H_{i}$ da equacào (4.87) empregaremos o teorema de Cayley.Hamilton (veja apendice B). O lado esquerdo desta equaçăo tem a forma $S_{p l}=\left(\alpha_{1} \delta_{p l} \div \varepsilon_{p m} H_{m}\right) H_{i}$ assin. vamos muluplicá-lo pelo tensor 


$$
\left(\mathrm{s}^{-1}\right)_{i p}=\frac{\left(\alpha_{1}^{2} \hat{\delta}_{i p}-\alpha_{1} \varepsilon_{i p k} \mathcal{H}_{k}+\mathcal{H}_{i} \mathcal{H}_{p}\right)}{\alpha_{1}\left(\alpha_{1}^{2}+\mathcal{H}^{2}\right)}
$$

e, obtemos que

$$
H_{i}^{w}=\frac{\alpha_{2}\left(\alpha_{1}^{2} \delta_{i p}-\alpha_{1} \varepsilon_{i p k} \mathcal{H}_{k}+\mathcal{H}_{i} \mathcal{H}_{p}\right)}{\alpha_{1}\left(\alpha_{1}^{2}+\mathcal{H}^{2}\right)} \frac{\partial T}{\partial x_{p}}
$$

Das equaçäes $(4.81),(4.86)$ e (4.91) resulta que o fluxo de calor reduzido $Q_{i}=Q_{i}^{T}+Q_{i}^{R}$, é dado por

$$
Q_{i}=\left[\beta_{1} \delta_{i j}+\beta_{2}\left(\alpha_{1} \varepsilon_{i j k} \mathcal{H}_{k}-\mathcal{H}_{i} \mathcal{H}_{j}\right)\right] \frac{\partial T}{\partial x_{j}}
$$

onde introduzimos

$$
\beta_{1}(\mathcal{H})=\frac{b_{3}}{b_{1}}+\frac{\left(b_{1}-b_{2}\right)}{b_{1}\left(a_{3} b_{1}-a_{2} b_{2}\right)}\left[\left(a_{4} b_{1}-a_{2} b_{3}\right)-\frac{a_{1} b_{1} \alpha_{1} \alpha_{2}}{\left(\alpha_{1}^{2}+\mathcal{H}^{2}\right)}\right]
$$

e

$$
\beta_{2}(\mathcal{H})=\frac{a_{1} \alpha_{2}\left(b_{1}-b_{2}\right)}{\alpha_{1}\left(\alpha_{1}^{2}+\mathcal{H}^{2}\right)\left(a_{3} b_{1}-a_{2} b_{2}\right)} .
$$

De acordo com a lei de Fourier generalizada $(4.69)$ e através da equaçäo $(4.92)$, o tensor condutividade térmica reduzido é dado por

$$
\lambda_{i j}(\mathcal{H})=-\left[\beta_{1} \hat{b}_{i j}+\beta_{2}\left(\alpha_{1} \varepsilon_{i j k} \mathcal{H}_{t}-\mathcal{H}_{i} \mathcal{H}_{j}\right)\right]
$$

Na ausência de campó magnético o coeficiente de condutividade térmica $\lambda_{i j}(0)$ é isotrópico e dado por

$$
\lambda_{i j}(0)=-\beta_{1}(0) \delta_{i j}=\lambda_{0} \delta_{i j}
$$

onde o escalar

$$
\lambda_{0}=\frac{3}{16} \frac{(\kappa+1)^{2}\left[24\left(50 \kappa^{2}+151 \kappa+37\right)+25 \pi \kappa\right]}{\left[8\left(102 \kappa^{3}+101 \kappa^{2}+75 \kappa+12\right)+\pi \kappa(17 \kappa+4)\right]} .
$$

é obtido da eqquaçăo (4.93). Assim, com a equação (4.78), podemos escrever para o coeficiente de condutividade térmica para $\mathcal{H}=0$ :

$$
\lambda_{0}^{\prime}=\frac{3}{16 a^{2}}\left(\frac{k^{3} T}{\pi m}\right)^{1 / 2} \frac{(\kappa+1)^{2}\left[24\left(50 \kappa^{2}+151 \kappa+37\right)+25 \pi \kappa\right\}}{\left[8\left(102 \kappa^{3}+101 \kappa^{2}+75 \kappa+12\right)+\pi \kappa(17 \kappa+4)\right]} .
$$




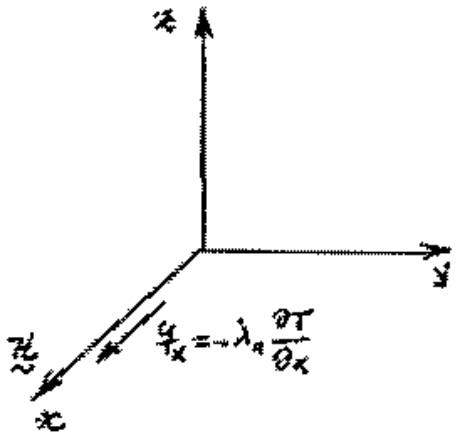

(ax)

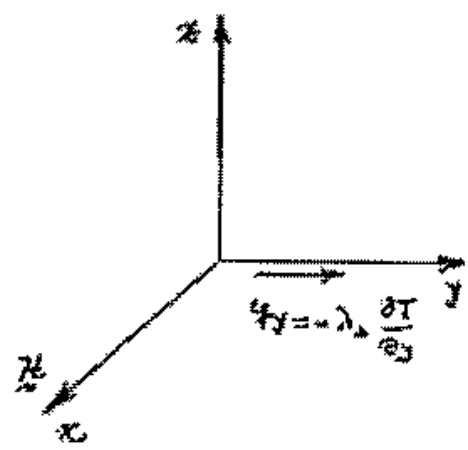

(b)

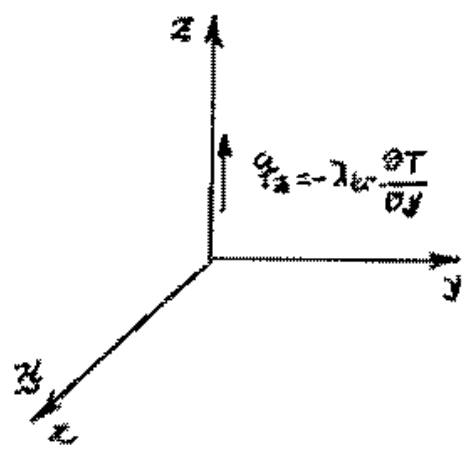

(c)

Figura 4.1: Os coeficientes de condutividade térmica: (a) paralelo, (b) perpendicular e (c) transversal.

- qual apresenta termos adicionais em relação ao coeficiente de condutividade têrmica dado pela equaşã̃o (4.78).

Sem perda de generalidade, consideremos em seguida, o campo magnético $\mathcal{H}$ coincidente com a direçào $x$ de um sisterna de coordenadas cartesianas com a origern fixada em algum ponto do gás. Para o tensor condutividade térmica, a partir da eqquaçào (4.95), temos a seguinte representação cattesiana [60]:

$$
(\boldsymbol{\lambda})_{i j}=\left(\begin{array}{lll}
\lambda_{x x} & 0 & 0 \\
0 & \lambda_{y y} & \lambda_{y z} \\
0 & -\lambda_{y z} & \lambda_{y y}
\end{array}\right)
$$

onde

$$
\begin{gathered}
\lambda_{x x}=-\left(\beta_{1}-\beta_{2} h^{2}\right) \\
\lambda_{y y}=\lambda_{z z}=-\beta_{1} \\
\lambda_{y z}=-\lambda_{z y}=-\alpha_{1} \beta_{2} \mu .
\end{gathered}
$$

Vamos introduzir os coeficientes de condutividade térmica paraleio $\left(\lambda_{11}\right)$, perpendicular $\left(\lambda_{1}\right)$ e transversal $\left(\lambda_{3 r}\right)$. Os coeficientes $\lambda_{\mid l}$ e $\lambda_{\perp}$ são obtidos pela medida do fluxo de calor na direçào do gradiente de temperatura $\nabla T$, com o campo magnético $\mathcal{H}$ aplicado respectivamente, nas direçóes paralela e perpendicular a $\nabla T$. O coeficiente $\lambda_{t r}$ é obtido medindo-se o fuxxo de calor na direçào que é mutuamente perpendicular (transversal) a $\nabla T$ e $\mathcal{H}$. Assim, na fig 4.1 temos: em (a) $q_{z}=-\lambda_{\|} \frac{\partial T}{\partial x}, \mathrm{em}(\mathrm{b}) q_{y}=-\lambda_{\perp} \frac{\partial T}{\partial y} \mathrm{em}(\mathrm{c}) q_{z}=-\lambda_{t} \frac{\partial T}{\partial y}$. 
Daste modo, a representaçäo da equaçäo (4.99), torna-se

$$
(\lambda)_{i j}=\left(\begin{array}{ccc}
\lambda_{\|} & 0 & 0 \\
0 & \lambda_{2} & \lambda_{t r} \\
0 & -\lambda_{t r} & \lambda_{1}
\end{array}\right)
$$

onde as três componentes independentes do tensor condutividade térmica, são dadas por

$$
\begin{aligned}
& \lambda_{\|}(H)=\lambda_{x x}=-\left(\beta_{1}-\beta_{2} \mathcal{H}^{2}\right)
\end{aligned}
$$

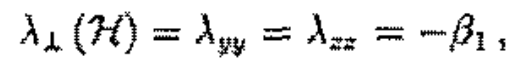

$$
\begin{aligned}
& \lambda_{t r}(H)=\lambda_{y z}=-\lambda_{2 y}=-\alpha_{1} \beta_{2} H
\end{aligned}
$$

Para $H=0$, temos: $\lambda_{1}(0)=\lambda_{\perp}(0)=\lambda_{0}$ e $\lambda_{t r}(0)=0$, com $\lambda_{0}$ dado pela equactao $(4.97)$; assim. dà equaçăo (4.103) decorre que

$$
\lambda_{0}=\frac{1}{3} \lambda_{\mathrm{rr}}(0)=\frac{1}{3}\left[\lambda_{1}(0)+2 \lambda_{1}(0)\right] .
$$

Estamos interessados em analisar as variaçöes relativas dos coeficientes de condutividade térmica dependernte do campo magnético externo $\mathcal{H}$, isto é, na forma

$$
\frac{\Delta \lambda_{i j}(H)}{\lambda_{0}}=\frac{\lambda_{i j}(\mathcal{H})-\lambda_{0} \delta_{i j}}{\lambda_{0}}
$$

Portanto, das equaços (4.104) e (4.105) temos, para o coeficiente de condutividade térmica paralelo

$$
\frac{\Delta \lambda_{11}(\mathcal{H})}{\lambda_{0}}=-\frac{\beta_{1}(\mathcal{H})-\mathcal{K}^{2} \beta_{2}(\mathcal{H})+\lambda_{0}}{\lambda_{0}}
$$

$e_{\text {s }}$ para o coeficiente de condutividade térmica perpendicular

$$
\frac{\Delta \lambda_{1}(\mathcal{H})}{\lambda_{0}}=-\frac{\beta_{1}\left(H_{0}+\lambda_{0}\right.}{\lambda_{0}}
$$

Para o coeficiente de condutividade térmica transversal verificamos, da equação (4.106), que $\lambda_{i r}(0)=0 ;$ assim, para este coeftciente nos interessa a variacäto relativa

$$
\frac{\lambda_{t r}(\mathcal{H})}{\lambda_{0}}=-\frac{\alpha_{1} \mathcal{H} \beta_{2}(\mathcal{H})}{\lambda_{0}}
$$

com $\lambda_{0}$ é dado pela equaçăo (4.97).

Com substituiçào das equaçoes $(4.93),(4.94)$ e $(4.97)$ na $(4.109)$, obtomos 


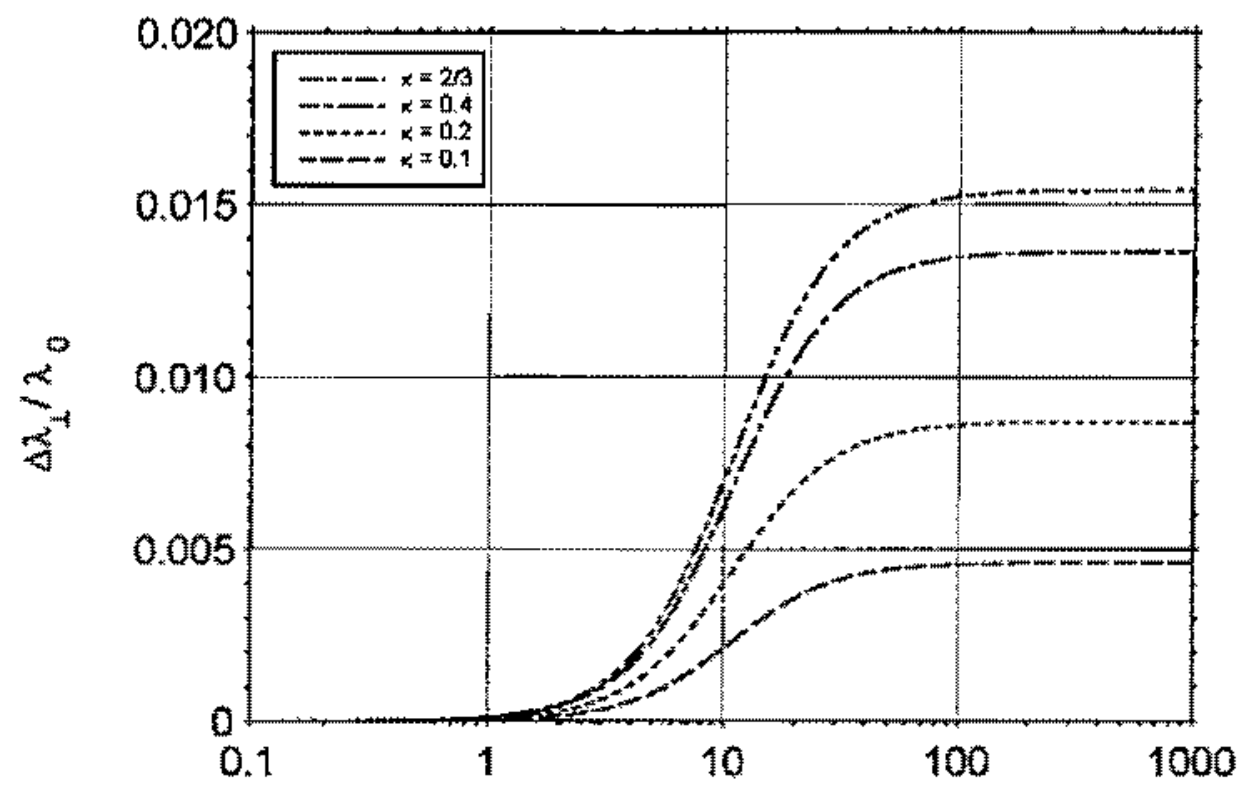

Parâmetro do Campo Magnético $(H)$

Figura 4.2: Variações relativas do coeficiente de condutividade térmica perpendicular

$$
\frac{\Delta \lambda_{\|}(r)}{\lambda_{0}}=0
$$

Portanto, neste modelo onde consideramos somente polarizaça $\varepsilon_{\text {; }} \Omega_{j} C_{k}$ o coeficiente de condutividade térmica na direçăo do campo magnëtico aplicado näo é afetado pelo campo. No entanto, dados experimentais [16] mostram que esta dependencia existe de modo que o modelo empregado não é completamente adequado.

As variaçotes relativas representadas pelas equacōes $(4.110)$ e (4.111) são mostradas, respectivamente, nas figuras 4.2 e 4.3, para alguns valores do momento de inércia adimensional $\kappa=\frac{4 l}{m_{a}^{2}}$ as quais säo da ordem de 1,0\%. No entanto como o modelo molecular de esferas rugosas superestima os valores para os coeficientes de transporte a contribuiçăo do campo $h_{i}(x, t)$ deve ser, na verdade, muito menor [5ia].

Desta forma, somente a polarizaçào representada pelo vetor de Waldmann $H_{i}(x, t)$ năo suficiente para descrever os coeficientes de condutividade térmica para um gás poliatômico sob um campo magnético aplicado.

Na próxima seção discutiremos a influéncia da polarização $\Omega_{<i} \Omega_{j>} C_{k}$, representada pelo t.ment $H_{<i, j}(x, 1)$. sobre estes conficientes, a qual mostrar-se-á mais realista diante dos dados experimentais. 


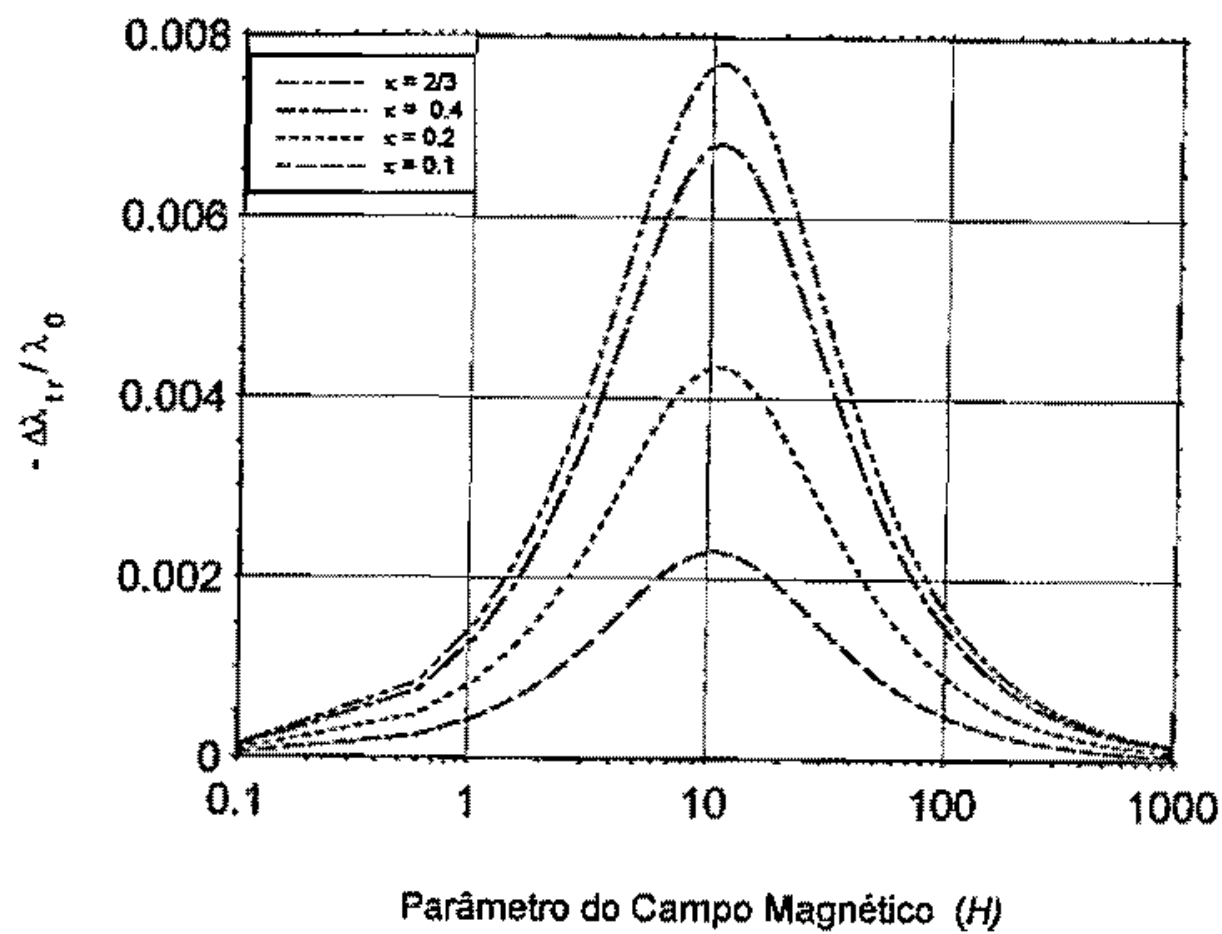

Figura 4.3: Variagöes relativas do coeficiente de condutividade termica transversal

\subsubsection{Campo $H_{<i j>k}(\mathrm{x}, t)$ e Coeficientes de Condutividade Térmica}

Consideremos em seguida, a influência da polarizaçäa $\Omega_{j} \Omega_{j} C_{k}$ sobre os coeficientes de condutividade térmica atrayés do campo $H_{\langle i j\rangle k}(x, t)$, definido segundo as equaçōes equaçōes $(4.17)$ e $(4.61)_{2}$ : por

$$
H_{<i j>k}=\frac{\sqrt{\pi} a^{2} T}{m}\left(\frac{m}{k T}\right)^{3 / 2} \int I \Omega_{\langle i} \Omega_{j\rangle} C_{k} \int d \mathrm{c} d \omega .
$$

Para os campos $Q_{t}^{T}\left(x_{1} t\right), Q_{i}^{R}(x, t)$ e $H_{\langle i j>k}(x, t)$ o sistema de equaçöes $(4.64)$ a $(4.67)$ se reduz a

$$
\begin{aligned}
& A_{1} H_{\langle i \backslash\rangle}+A_{2} Q_{i}^{T}+A_{3} Q_{i}^{R}=A_{4} \frac{\partial T}{\partial x_{i}} \\
& B_{1} H_{<i b\rangle}+B_{2} Q_{i}^{T}+B_{3} Q_{i}^{R}=B_{4} \frac{\partial T}{\partial x_{i}} \\
& {\left[D_{1} \delta_{i j} \delta_{p k}-D_{2}\left(\delta_{i k} \delta_{p j}+\delta_{j k} \delta_{i p}\right)\right] H_{\langle i p\rangle]}-D_{3} H_{\langle i j\rangle k}-D_{4}\left(H_{\langle i k\rangle j}+H_{\langle j k\rangle j}\right)} \\
& -D_{3}\left(\delta_{i k} \delta_{p j}+\delta_{j k} \delta_{t p}-\frac{2}{3} \delta_{i j} \delta_{k p}\right) Q_{p}^{T}+D_{6}\left(\delta_{i k} \delta_{j s}+\delta_{j k} \delta_{i s}-\frac{2}{3} \delta_{i j} \delta_{k s}\right) Q^{k}
\end{aligned}
$$




$$
=D_{7}\left(\delta_{i i} \varepsilon_{p j r}+\delta_{j i t_{p i r}}\right) H_{<p l>k} \mathcal{H}_{*},
$$

onde introduzimos os coeficientes

$$
\begin{array}{cl}
A_{1}=3(2 \kappa+1), & A_{2}=-10 \kappa, A_{3}=10\left(2 \kappa^{2}+2 \kappa+1\right), \quad A_{4}=-\frac{45}{8}(\kappa+1)^{2}, \\
B_{1}=15 \kappa, & B_{2}=6(17 \kappa+4), \quad B_{3}=-50 \kappa, \quad B_{4}=-\frac{225}{8}(\kappa+1)^{2}, \\
D_{1}=4(2 \kappa+1), & D_{2}=11 \kappa+3, \quad D_{3}=2\left(20 \kappa^{2}+16 \kappa+11\right), D_{4}=\kappa+3, \\
D_{5}=4 \kappa . & D_{6}=-4(2 \kappa+1), \quad D_{3}=\frac{15}{2}(\kappa+1)^{2} .
\end{array}
$$

Nosso objetivo é resolver o sisterna de equaçóes $(4.114)$ a (4.116) para os campos $Q_{i}^{R}(\mathcal{H})$ e $Q_{i}^{T}(\mathcal{H})$, de modo a obter os coeficientes de condutividade térmica $\lambda_{i j}$ através das equaçóes $(4.68)$ e $(4.69)$.

0 procedimento que empregaremos consiste em resolver primeiramente o sistema (4.114) a (4.116) para $\mathcal{H}=0$ e então, após subtrair do sistema original as equações de campo nulo, resolver o sistema de equaçôes dependentes do campo magnético [51]. Para isso, vamos escrever os campos na forma

$$
\begin{gathered}
H_{<i,>k}(\mathcal{H})=H_{<i,>k}^{(0)}+H_{<i j>k}^{(1)}(\mathcal{H}) \\
Q_{i}^{T}(\mathcal{H})=Q_{i}^{T^{(0)}}+Q_{i}^{T^{(1)}}(\mathcal{H}) \\
Q_{i}^{R}(\mathcal{H})=Q_{i}^{R(0)}+Q_{i}^{R(1)}(\mathcal{H})
\end{gathered}
$$

sendo que

$$
\begin{gathered}
H_{<j>k}^{(1)}(0)=0 \\
Q_{i}^{r^{(1)}}(0)=0 \quad \text { e } \quad Q_{i}^{R(1)}(0)=0 .
\end{gathered}
$$

\subsubsection{Coeficiente de Condutividade Térmica para Campo Magnético Nulo}

Com o objetivo de resolver o sistema de equaçōes (4.114) a (4.116) vamos inicialmente. obier a solução deste sistema para $\mathcal{H}=0$. Assim. temos 


$$
\begin{aligned}
& A_{1} H_{<i>l}^{(0)}+A_{2} Q_{i}^{T^{(0)}}+A_{3} Q_{i}^{p^{(0)}}=A_{4} \frac{\partial T}{\partial x_{i}} \\
& B_{1} H_{\langle t\rangle)}^{(0)}+B_{2} Q^{T(0)}+B_{3} Q_{i}^{R(0)}=B_{4} \frac{\partial T}{\partial x_{i}} \\
& {\left[D_{1} \delta_{i j} \delta p k-D_{2}\left(\delta_{i k} \delta_{p j}+\delta_{j k} \delta_{i p}\right)\right] H_{<y 1>1}^{(0)}-D_{3} H_{<i j>k}^{(0)}-D_{4}\left(H_{<i k>j}^{(0)}+H_{<j k>i}^{(0)}\right)-} \\
& D_{5}\left(\delta_{i k} \delta_{i p}+\delta_{j k} \delta_{i p}-\frac{2}{3} \delta_{i j} \delta_{k p}\right) Q_{y}^{T(0)}+D_{6}\left(\delta_{i k} \delta_{j y}+\delta_{j k} \delta_{i s}-\frac{2}{3} \delta_{i j} \delta_{k s}\right) Q_{s}^{R(0)}=0 .
\end{aligned}
$$

Resolvendo este sistema de equacoes para $Q_{i}^{T^{(0)}}$ e $Q^{n^{(0)}}$, resulta para o fuxo de calor translacional reduzido

$$
Q_{i}^{T^{(0)}}=E_{1} H_{<i l>1}^{(0)}+E_{3}^{z} \frac{\partial T}{\partial x_{i}}
$$

e, para o fluxo de calor rotacional reduzido

$$
Q_{i}^{n(0)}=E_{2} H_{<i l i l}^{(0)}+E_{4} \frac{\partial T}{\partial x_{i}}
$$

onde introduzimos os coeficientes

$$
\begin{array}{ll}
E_{1}=\frac{A_{3} B_{1}-A_{1} B_{3}}{A_{2} B_{3}-A_{3} B_{2}}, & E_{3}=\frac{A_{4} B_{3}-A_{3} B_{4}}{A_{2} B_{3}-A_{3} B_{2}}, \\
E_{2}=\frac{A_{1} B_{3}-A_{2} B_{1}}{A_{2} B_{3}-A_{3} B_{2}}, & E_{4}=\frac{A_{2} B_{4}-A_{4} B_{2}}{A_{2} B_{3}-A_{3} B_{2}}
\end{array}
$$

Consideremos o vetor $H_{<i, j}^{\text {to }}$ proporcional ao gradiente de temperatura, ou seja

$$
H_{<i l>l}^{(0)}=\frac{10}{3} \beta_{1} \frac{\partial T}{\partial x_{i}}
$$

onde $y_{1}$ é um parâmetro a detetminar e independente do campo magnético $\mathcal{H}$. Esta hipótese será justificada na seçấo seguinte [veja a equação (4.150)].

$P$ ara determinar $\beta_{1}$ vamos multiplicar a equaçäo (4.12) por $\delta_{j k}$ e na equacto resultante. substituinos as equaçós $(4.128)$ e (4.129); obtemos, entào

$$
\left(D_{1}-4 D_{2}-D_{3}-D_{4}-\frac{10}{3} D_{5} E_{1}+\frac{10}{3} E_{4} D_{6}\right) H_{<i b l}^{(0)}=\frac{10}{3}\left(D_{5} E_{3}-D_{6} E_{4}\right) \frac{\partial T}{\partial x_{i}}
$$

e. comparando corn a equaçăo (4.131), concluimos que

$$
H_{1}=\frac{D_{5} E_{3}-D_{6} E_{4}}{D_{1}-4 D_{2}-D_{3}-D_{4}-\frac{10}{3}\left(D_{5} E_{1}-E_{2} D_{5}\right)}
$$


Desta forma, os fluxos de calor reduzidos podem ser escritos, através das equaçöes (4.12S) e (4.129), como

$$
Q_{t}^{r(0)}=\left(E_{3}+\frac{10}{3} E_{1} \beta_{1}\right) \frac{\partial T}{\partial x_{i}}
$$

e

$$
Q_{i}^{n(0)}=\left(E_{4}+\frac{10}{3} E_{2} \beta_{1}\right) \frac{\partial T}{\partial x_{i}}
$$

As duas últimas equaçôes expressam a lei de Fourier $Q=-\lambda \frac{\partial T}{\partial x_{i}}$, para os fuxos de calor reduzidos translacional e rotacional, respectivamente.

Através das equaçōes $(4.117)$ a $(4.119),(4.130)$ e $(4.133)$ a $(4.135)$ podemos escrever para o coeficiente de condutividade térmica translacional reduzido

$$
\lambda_{0}^{T}=\frac{75}{16} \frac{(\alpha+1)^{3}\left(80 x^{2}+98 x+29\right)}{\left(1360 x^{4}+2266 \kappa^{3}+1707 \kappa^{2}+853 x+116\right)}
$$

para o coeficiente de condutividade térmica rotacional reduzido

$$
\lambda_{0}^{R}=\frac{3}{4} \frac{\left(760 \kappa^{4}+2216 \kappa^{3}+2315 \kappa^{2}+958 \kappa+99\right)}{\left(1360 \kappa^{4}+2260 \kappa^{3}+1707 \kappa^{2}+853 \kappa+116\right)}
$$

de modo que o coeficiente de condutividade térmica reduzido é dado por

$$
A_{0}=\lambda_{0}^{T}+\lambda_{0}^{R}=\frac{3}{16} \frac{(\kappa+1)^{2}\left(2000 \kappa^{4}+9490 \kappa^{3}+13449 \kappa^{2}+7336 \kappa+1121\right)}{\left(1360 \kappa^{5}+3626 \kappa^{4}+3973 \kappa^{3}+2560 \kappa^{2}+969 \kappa+116\right)}
$$

Portanto, o coficiente de condutividade térmica para o gás poliatômico pode ser escrito, através da equacoes $(4.70)$ e $(4.138)$, como

$$
H_{0}=\frac{3}{16} \frac{1}{a^{2}}\left(\frac{k^{3} T}{\pi T H}\right)^{1 / 2} \frac{(\kappa+1)^{2}\left(2000 \kappa^{4}+9490 \kappa^{3}+13449 \kappa^{2}+7336 \kappa+1121\right)}{\left(1360 \kappa^{5}+3626 \kappa^{4}+3973 \kappa^{3}+2560 \kappa^{2}+969 \kappa+116\right)} .
$$

Este resultado foi obtido anteriormente por Dahler e colaboradores [61].

\subsubsection{Influência do Carnpo Magnético Aplicado nos Coeficientes Condutividade Termica}

Conforme já observarnos na seçầ 4.5 .2 , sob a açäo de um campo magnético externo um gás poliatömico tornawse anisotrópíco, de modo que há três coeficientes independentes de condutividade térmica para o gás. 
Nesta seção vamos considerar a influência da polarizaçăo $\Omega_{<i} \Omega_{j} C_{k}$ atravếs do campo básico $H_{<i j\rangle k}$, definido pela equatăo (4.113) e que satisfaz ao sistema de equaços (4.114) a (4.116), sobre os coeficientes de condutividade térmica. Na ausência de campo magnético externo a gás é isotrópico com o coeficiente da condutividade térmica dado pela equaçäo (4.139).

Se subtrairmos as equaçós para campo nulo (4.125) a (4.127) das equaçós correspondentes $(4.114)$ a $(4.116)$ e, tendo cm conta as equaçoes $(4.120)$ a $(4.122)$, obtemos

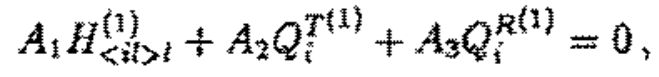

$$
\begin{aligned}
& B_{1} H_{\langle i(>1}^{(1)}+B_{2} Q_{i}^{T(1)}+B_{3} Q_{i}^{F(1)}=0 \\
& {\left[D_{1} \delta_{i j} \delta_{p k}-D_{2}\left(\delta_{i k} \delta_{p j}+\delta_{j k} \delta_{i p}\right)\right] H_{\langle p i>k}^{(1)}-D_{3} H_{\langle i j>k}^{(1)}-D_{4}\left(H_{<i k>j}^{(1)}+H_{<j k>i}^{(1)}\right)}
\end{aligned}
$$

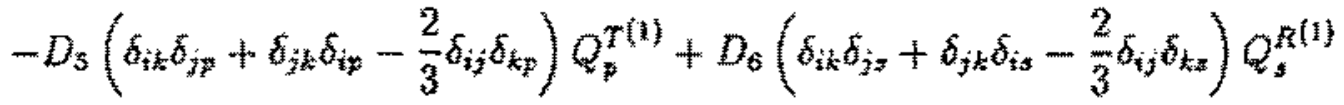

$$
\begin{aligned}
& =D_{7}\left(\varepsilon_{p j r} \delta_{i l}+\varepsilon_{p i r} \delta_{\eta l}\right)\left(H_{\langle p t>k}^{(0)}+H_{\langle p l>k}^{(1)}\right) H_{r}
\end{aligned}
$$

com os coeficientes $A_{i}, B_{i}$ e $D_{i}$ dados pelas equaçöes $(4.117)$ a $(4,119)$.

Resolvendo as duas primeiras equaçôes deste sistema para $Q_{i}^{T^{(1)}}$ e $Q_{i}^{R^{(1)}}$, obtemos

$$
Q_{i}^{T(1)}=E_{1} H_{<i b i}^{(1)}
$$

e

$$
Q^{R(1)}=E_{z} H_{<i l i t}^{(1)}
$$

que representam, respectivamente, os fluxos de calor reduzidos transiacional e rotacional dependentes do campo magnético. $E_{1}$ e $E_{2}$ säo definidos pelas equaçóes $(4,130)_{1,2}$.

A comparaçäo das duas últimas equaröos com a (4.69) indica que para determinarmos o vetor tluxo de calor dependente do campo magnético

$$
Q_{i}^{(1)}=Q^{r(1)}+Q_{i}^{(n)}=\left(E_{1}+E_{2}\right) H_{\langle i\rangle}^{(1)}
$$

e em cousequếncia, os coeficientes de condulividade térmica, devemos obter o vetor $H_{\text {cit> }}$ como funçäo do campo magnético reduzido $\mathcal{H}$ e do gradiente de temperatura $\nabla T$. Este vetor decorre da contraçäo de doís indices do tensor $H_{<i j>k}$, o qual deve ser determinado a partir da equaçäo (4.142).

Substituindo as equaçóes (4.143) e (4.144) na (4.142) e rearranjando os termos, obtemos 


$$
\begin{aligned}
& {\left[D_{1} \delta_{i j} \delta_{7 k}-D_{2}\left(\delta_{i k} \delta_{p y}+\delta_{j k} \delta_{i p}\right)+\left(E_{2} D_{6}-E_{1} D_{5}\right)\left(\delta_{i k} \delta_{j p}+\delta_{i k} \delta_{i p}-\frac{2}{3} \delta_{i j} \delta_{k p}\right)\right] H_{\langle p l\rangle i}^{(1) j}} \\
& -D_{3} H_{\langle i j\rangle k}^{(1)}-D_{4}\left(H_{\langle i k>j\rangle}^{(1)}+H_{\langle j k>i}^{(1)}\right)=D_{7}\left(\delta_{i i E_{p j \tau}}+\delta_{j t E_{n i \gamma}}\right) H_{*}\left(H_{\langle p i>k}^{(0)}+H_{\langle p i>k}^{(1)}\right)
\end{aligned}
$$

que é uma equação tensorial para as componentes do tensor

$$
H_{\langle i j\rangle k}=H_{\langle i j\rangle k}^{(0)}+H_{\langle i\rangle>k}^{(1)}
$$

onde $H_{\langle i>k}^{(1)}(0)=0$.

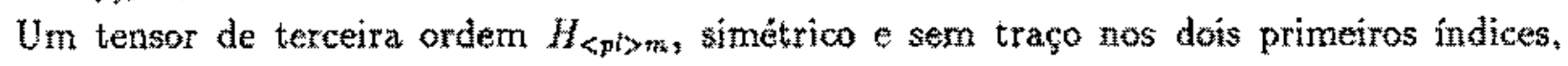
dependente do campo magnético $\mathcal{H}^{1}$ e do gradiente de temperatura $\nabla T$, tem a seguinte repre. sentação ( veja apêndíce $\mathrm{B}$ ):

$$
\begin{aligned}
& H_{<p\rangle m}=H_{<p l>m}^{(0)}+H_{<p l>m}^{(1)}=\int \beta_{1}\left(\delta_{p m} \delta_{i s}+\delta_{p s} \delta_{l m}-\frac{2}{3} \delta_{p h} \delta_{m s}\right)
\end{aligned}
$$

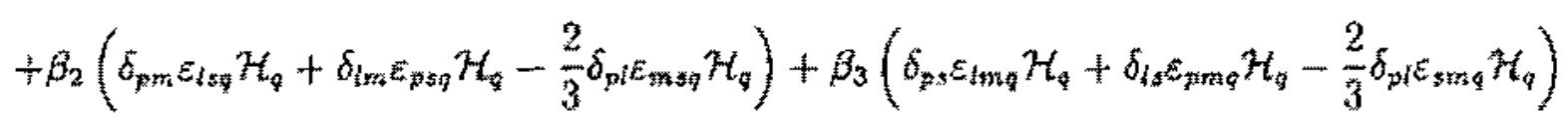

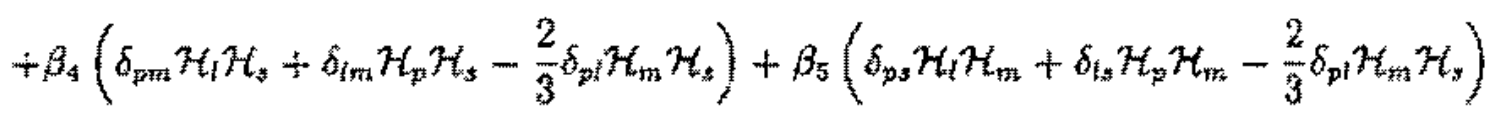

$$
\begin{aligned}
& +\beta_{s}\left(\tilde{\delta}_{m s} H_{p} H_{i}-\frac{1}{3} \delta_{p} \partial_{m s} H^{2}\right)+\beta_{z}\left(\varepsilon_{m m q} H_{q} H_{i}+\varepsilon_{m q} \mathcal{H}_{q} H_{p}\right) H_{s}
\end{aligned}
$$

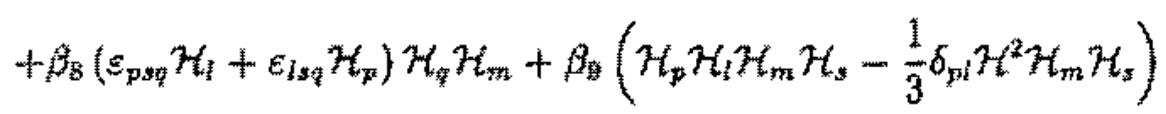

$$
\begin{aligned}
& \left.+\beta_{10}\left(\delta_{p m s} \delta_{t s}+\delta_{p s} \delta_{m}-\frac{2}{3} \delta_{p t} \delta_{m s}\right)\right] \frac{\partial T}{\partial x_{s}} .
\end{aligned}
$$

Nesta representaça on coeficientes $\beta_{i}=2,3, \ldots, 10$ são funções de potencias de $h^{2}$ enquanto que o coeficiente $\beta_{1}$ independe do campo magnético. De fato, para $\mathcal{H}=0$ a equąà $(4.147)$ se reduz a:

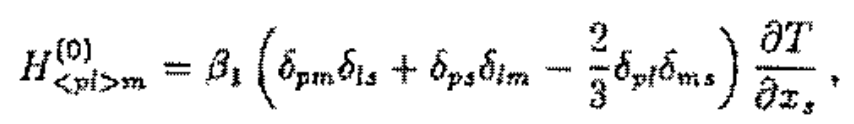

que é a representação para o tensor $H_{\langle p l\rangle m}^{t 0)}$ o qual depende, somente, do gradiente de tempera* tura VT.

Além disso, se multiplicarmos a equação anterior por $\delta_{\text {tm: }}$ obtemos

\footnotetext{
'Aa verdade, o lensor $H_{<p i>m}$ depende do campo magnélico através do tensor antissimétrico $B_{i j}=\varepsilon_{i j} H_{j}$ (veja apendic B).
} 


$$
H_{<p l>t}^{(0)}=\frac{10}{3} \beta_{1} \frac{\partial T}{\partial x_{p}}
$$

que é a equação (4.131) empregadá na seçâo 4.5.3.1. O coeficiente $\beta_{1}$ está, portanto, determinạdo através da equação (4.133).

Vamos reescrever o tensor $H_{<p l\rangle m}$ na forma

$$
H_{<p l>m}=t_{<\langle t>r q q} \frac{\partial T}{\partial x_{q}}=\left(t_{<p l>m q}^{(0)}+t_{<p l>m q}^{(1)}\right) \frac{\partial T}{\partial x_{q}}
$$

onde, das equações $(4.148)$ e (4.149), temos

$$
i_{\langle p l>m q}^{(0)}=\beta_{1}\left(\delta_{p m} \delta_{l q}+\delta_{p q} \delta_{i m}-\frac{2}{3} \delta_{p i} \delta_{m q}\right)
$$

$e_{2}$

$$
\begin{aligned}
& t_{<p l>m q}^{(1)}=\left[\beta_{2}\left(\delta_{p m i q} \varepsilon_{i q} \mathcal{H}_{r}+\delta_{l m} \varepsilon_{p q q} \mathcal{H}_{r}-\frac{2}{3} \delta_{p i} \varepsilon_{m q}, \mathcal{H}_{r}\right)\right.
\end{aligned}
$$

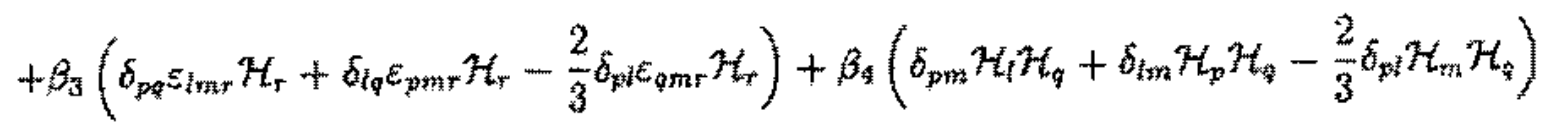

$$
\begin{aligned}
& +\beta_{s}\left(\delta_{p q} H_{i} H_{m}+\delta_{i q} \mathcal{H}_{p} \mathcal{H}_{m}-\frac{2}{3} \delta_{p} \mathcal{H}_{m} \mathcal{H}_{q}\right)+\beta_{\varepsilon}\left(\delta_{m q} \mathcal{H}_{p} \mathcal{H}_{t}-\frac{1}{3} \delta_{p t} \delta_{m q} \mathcal{H}^{2}\right) \\
& +\beta_{7}\left(\varepsilon_{p m r} \mathcal{H}_{r} \mathcal{H}_{t}+\varepsilon_{\text {mrr }} \mathcal{H}_{r} \mathcal{H}_{p}\right) \mathcal{H}_{q}+\beta_{8}\left(\varepsilon_{p q r} \mathcal{H}_{t}+\varepsilon_{q \mathrm{q}} \mathcal{H}_{p}\right) \mathcal{H}_{\mathrm{r}} \mathcal{H}_{m} \\
& \left.+\beta_{9}\left(\mathcal{H}_{p} \mathcal{H}_{i} \mathcal{H}_{m} \mathcal{H}_{q}-\frac{1}{3} \delta_{p i} \mathcal{H}^{2} \mathcal{H}_{m} \mathcal{H}_{q}\right)+\beta_{10}\left(\delta_{p m} \delta_{l q}+\delta_{p q} \delta_{l m}-\frac{2}{3} \delta_{p m} \delta_{i q}\right)\right] .
\end{aligned}
$$

Multiplicando a equação (4.146) por $\delta_{i j}$, obtemos a seguinte relação entre coeficientes

$$
3 D_{1}-2 D_{2}-2 D_{4}=0
$$

a qqual pode ser verficada através da equaçào (4.119).

Corn as equações $(4.151)$ e (4.154) podemos escrever a equação tensorial (4.146) como

$$
\begin{aligned}
& E\left(\delta_{i k} t_{\langle j>>m}^{(1)}+\delta_{j k} t_{\langle i r>r m}^{(1)}-\frac{2}{3} \delta_{i j} t_{\langle k+>r m}^{(1)}\right)-D_{3} t_{<i j>m m}^{(1)}-D_{4}\left(t_{\langle i k>j m}^{(1)}+t_{<j k>i m}^{(1)}-\frac{2}{3} \delta_{i j} t_{\langle k r>+m}^{(1)}\right)
\end{aligned}
$$

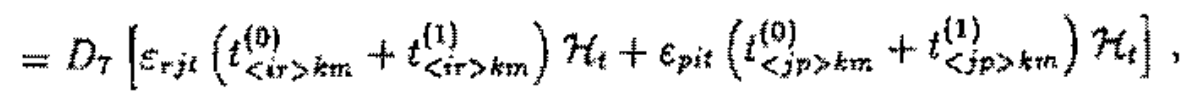

onde definimos

$$
E=-E_{1} D_{5}+E_{2} D_{6}-D_{2} \text {. }
$$

Substituindo as equaçōes $(4.152)$ e (4.153) na (4.155), resuka 


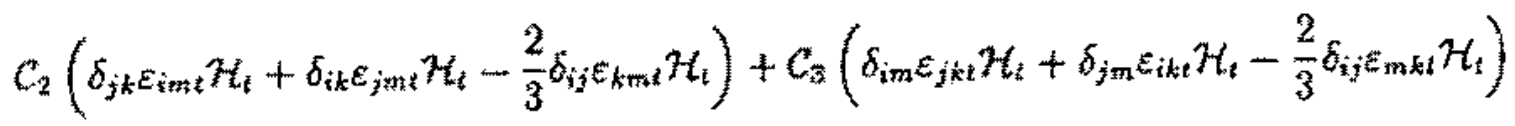

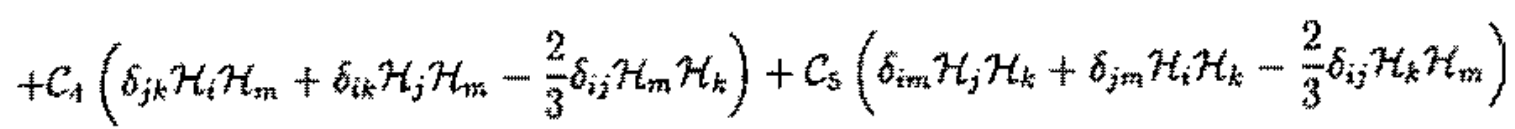

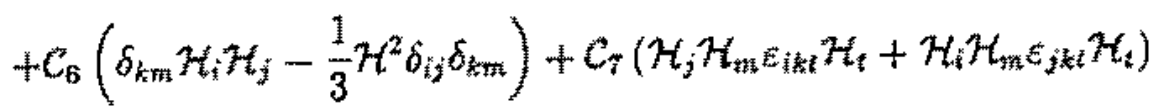

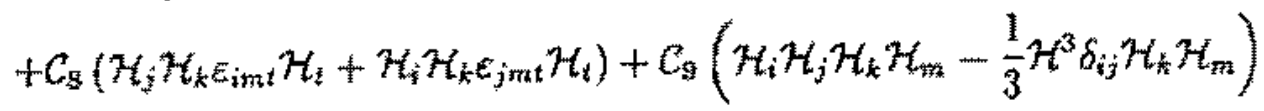

$$
\begin{aligned}
& +C_{10}\left(\delta_{i m} \delta_{j k}+\delta_{i k} \delta_{j m}-\frac{2}{3} \delta_{i j} \delta_{k m}\right)-2 D_{4} \beta_{8}\left(\mathcal{H}_{i} \mathcal{H}_{j} \varepsilon_{k m i} \mathcal{H}_{i}-\frac{1}{3} \mathcal{H}^{2} \delta_{j} \varepsilon_{k m i} \mathcal{H}_{i}\right)=0
\end{aligned}
$$

com os coeficientes $\mathcal{C}_{i}$ dados por:

$$
\begin{aligned}
& C_{2}=D_{2} \beta_{1}+\frac{1}{3}\left(10 E-3 D_{3}-D_{4}\right) \beta_{2}+\frac{1}{3}\left(5 E-2 D_{4}\right) \beta_{3}+E H^{2} \beta_{8}+D_{7} \beta_{10} \\
& c_{3}=D_{7} \beta_{1}+\left(D_{4}-D_{3}\right) \beta_{3}+D_{7} \beta_{10} \\
& C_{4}=2 D_{7} \beta_{2}+D_{7} \beta_{3}+\frac{1}{3}\left(10 E-3 D_{3}-D_{4}\right) \beta_{4}+\frac{1}{3}\left(2 D_{4}+E\right) \beta_{5} \\
& +E \beta_{6}-D_{7} \mathcal{H}^{2} \beta_{7}+\frac{1}{3} h^{2}\left(2 E+D_{4}\right) \beta_{9} \\
& \mathcal{C}_{5}=D_{7} \beta_{2}+2 D_{7} \beta_{3}-\left(D_{3}+D_{4}\right) \beta_{5}-D_{4} \beta_{6}-D_{7} h^{2} \beta_{3} \\
& C_{6}=-2 D_{7} \beta_{2}-2 D_{7} \beta_{3}-2 D_{4} \beta_{5}-D_{3} \beta_{6} \\
& C_{7}=D_{7} \beta_{4}+\left(D_{4}-D_{3}\right) A_{7} \\
& C_{8}=D_{7} \beta_{5}-\left(D_{3}+D_{4}\right) \beta_{8} \\
& c_{9}=2 D_{7} \beta_{7}+2 D_{7} \beta_{8}-\left(D_{3}+2 D_{4}\right) \beta_{9} \\
& \mathcal{C}_{10}=-2 D_{7} \mathcal{H}^{2} \beta_{2}-2 D_{7} \mathcal{H}^{2} \beta_{3}+E \mathcal{H}^{2} \beta_{3}+\frac{1}{3} H^{2}\left(D_{4}-E\right) \beta_{6} \\
& +\frac{1}{3}\left(10 E-3 D_{3}-D_{4}\right) \beta_{10}
\end{aligned}
$$


Podemos escrever o último termo da equaça (4.157) na seguinte forma:

$$
\begin{aligned}
& \mathcal{H}_{i} \mathcal{H}_{j} \varepsilon_{\text {tami }} \mathcal{H}_{t}-\frac{1}{3} \mathcal{H}^{2} \delta_{i j} \varepsilon_{k m i t} H_{t}= \\
& =\frac{1}{2}\left\{\mathcal{H}^{2}\left[\delta_{i m k} \varepsilon_{j k t} \mathcal{H}_{t}+\delta_{j m} \varepsilon_{i k l} \mathcal{H}_{t}-\frac{2}{3} \delta_{i j} \varepsilon_{m k l} \mathcal{H}_{t}\right]\right. \\
& -\mathcal{H}^{a}\left[\delta_{j k} \varepsilon_{i m t} H_{t}+\delta_{i k} \varepsilon_{j m i} H_{i}-\frac{2}{3} \delta_{i j} \varepsilon_{k m t} H_{i}\right] \\
& +\left[H_{k} H_{j} \varepsilon_{i m i} H_{i}+\mathcal{H}_{k} H_{i} \varepsilon_{j m t} H_{t}\right] \\
& \left.-\left[\mathcal{H}_{n} \mathcal{H}_{j} \varepsilon_{i k t} \mathcal{H}_{i}+\mathcal{H}_{i} \mathcal{H}_{m} \varepsilon_{j k i} \mathcal{H}_{t}\right]\right)
\end{aligned}
$$

e substituindo esta equaçäo na (4.15\%), resulta

$$
\begin{aligned}
& \left(c_{2}-D_{4} H^{2} h_{s}\right)\left(\delta_{j k} \varepsilon_{i m t} H_{i}+\delta_{i k} \varepsilon_{j m} H_{i}-\frac{2}{3} \delta_{i j} \varepsilon_{k m t} H_{t}\right) \\
& +\left(c_{3}+D_{4} \mathcal{H}^{2} \beta_{8}\right)\left(\delta_{m} \varepsilon_{j k t} \mathcal{H}_{t}+\delta_{3 m} \epsilon_{t k t} \mathcal{H}_{t}-\frac{2}{3} \delta_{t j} \varepsilon_{m k t} \mathcal{H}_{t}\right) \\
& +C_{4}\left(H_{i} H_{m} \delta_{j k}+H_{j} H_{m} \delta_{i k}-\frac{2}{3} H_{m k} H_{k} \delta_{i j}\right) \\
& +\mathcal{C}_{5}\left(\mathcal{H}_{j} \mathcal{H}_{k} \delta_{i m}+\mathcal{H}_{i} \mathcal{H}_{k} \delta_{m}-\frac{2}{3} \mathcal{H}_{k} \mathcal{H}_{m} \delta_{i j}\right) \\
& +c_{6}\left(\mathcal{K}_{i} H_{j} \delta_{k \ldots}-\frac{1}{3} \mathcal{H}^{2} \delta_{i j} \delta_{k+k}\right) \\
& +\left(C_{7}-D_{4} \delta_{s}\right)\left(\mathcal{H}_{\mathrm{m}} \mathcal{H}_{j} \varepsilon_{i k l} \mathcal{H}_{i}+\mathcal{H}_{i} \mathcal{H}_{\mathrm{m}} \varepsilon_{j k} \mathcal{H}_{t}\right) \\
& +\left(C_{8}+D_{4} \rho_{s}\right)\left(\mathcal{H}_{j} \mathcal{H}_{k} \varepsilon_{\text {int }} \mathcal{H}_{t}+\mathcal{H}_{i} \mathcal{H}_{k} \varepsilon_{j m i} \mathcal{H}_{i}\right)
\end{aligned}
$$

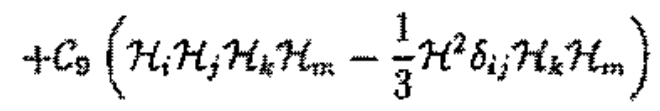

$$
\begin{aligned}
& +\mathcal{C}_{10}\left(\delta_{i m} \delta_{i k}+\delta_{i k} \delta_{j m}-\frac{2}{3} \delta_{i j} \hat{\delta}_{k m}\right)=0
\end{aligned}
$$

O primeiro membro desta equaçăo é constituido de termos tensoriais linearmente independentes. como podemos verificar da equação (4.153). Assim, se igualarmos os coeficientes da equação (4.168) a zero e com as equaçōes $(4.158)$ a $(4.166)$, abtemos o seguinte sistema de equaços para $\beta_{i}, i=2, \ldots, 10$ :

$$
\begin{gathered}
\left(10 E-3 D_{3}-D_{4}\right) \beta_{2}+\left(5 E^{2}-2 D_{4}\right) \beta_{3}+3 \mathcal{H}^{2}\left(D_{4}+E\right) \beta_{8}+3 D_{7} \beta_{10}=-3 \beta_{1} D_{r} \\
\left(D_{4}-D_{3}\right) \beta_{3}-D_{4} \mathcal{H}^{2} \beta_{8}+D_{7} \beta_{10}=-\beta_{1} D_{7}
\end{gathered}
$$




$$
\begin{aligned}
& 3 D_{7}\left(2 \beta_{2}+\beta_{3}\right)+\left(10 E-3 D_{3}-D_{4}\right) \beta_{4}+\left(2 D_{4}+E\right) \beta_{5}+3 E \partial_{6} \\
& -3 D_{7} \mathcal{K}^{2} \beta_{7}+\mathcal{H}^{2}\left(2 E+D_{4}\right) \beta_{5}=0 \\
& D_{7}\left(\beta_{2}+2 \beta_{3}\right)-\left(D_{3}+D_{4}\right) \beta_{5}-D_{4} \beta_{3}-D_{7} K^{2} \beta_{3}=0 \\
& 2 D_{7} \beta_{2}+2 D_{7} \beta_{3}+2 D_{4} \beta_{5}+D_{3} \beta_{6}=0 \\
& D_{7} \beta_{4}+\left(D_{4}-D_{3}\right) B_{7}+D_{1} B_{8}=0 \\
& D_{7} \rho_{5}-\left(2 D_{4}+D_{3}\right) \beta_{8}=0 \\
& 2 D_{7}\left(\beta_{7}+\beta_{5}\right)-\left(2 D_{4}+D_{3}\right) \beta_{2}=0 \\
& -6 D_{7} H^{2}\left(\beta_{2}+\beta_{3}\right)+3 E \mathcal{H}^{2} \beta_{5}+H^{2}\left(D_{4}-E\right) \beta_{6} \\
& +\left(10 E-3 D_{3}-D_{4}\right) \beta_{10}=0
\end{aligned}
$$

$\operatorname{com} \beta_{1}$ dado pela equação $(4,133)$.

Da equacto $(4.153)$ Lemos

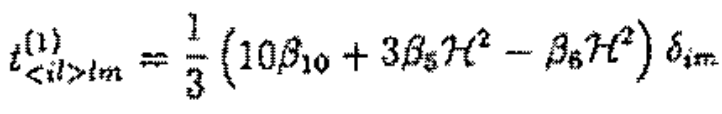

$$
\begin{aligned}
& +\frac{1}{3}\left(10 \beta_{4}+\beta_{3}+3 \beta_{6}+2 \beta_{3} \mathcal{H}^{2}\right) \mathcal{H}_{i} \mathcal{H}_{m} \\
& +\frac{1}{3}\left(10 \beta_{2}+5 \beta_{3}+3 \beta_{8} H^{2}\right) E_{m s} H_{t}
\end{aligned}
$$

e o vetor fuxo de calor total dependente do carmpo magnético, dado pela equaçăo (4.145), pode ser escrito como

$$
\begin{gathered}
Q_{i}^{(1)}=\left(E_{1}+E_{2}\right) t_{\langle i, i m}^{(1)} \frac{\partial T}{\partial x_{m i}}= \\
=\frac{1}{3}\left(E_{1}+E_{2}\right)\left[\left(10 \beta_{10}+3 \beta_{5} \mathcal{H}^{2}-\beta_{6} H^{2}\right) \delta_{i m}\right. \\
+\left(10 \beta_{4}+\beta_{5}+3 \beta_{6}+2 \beta_{s} \mathcal{H}^{2}\right) \mathcal{H}_{i} \mathcal{H}_{m}
\end{gathered}
$$




$$
\left.+\left(10 \beta_{2}+5 \beta_{3}+3 \beta_{8} H^{2}\right) \varepsilon_{\text {ini }} H_{k}\right] \frac{\partial T}{\partial x_{\mathrm{m}}}
$$

Assim, podemos escrever o tensor condutividade térmica reduzido dependente do campo magnético, através da equação (4.69), como

$$
\begin{gathered}
\lambda_{i j}^{(1)}(\mathcal{H})=\lambda_{i j}(\mathcal{H})-\lambda_{0} \tilde{o}_{i j}= \\
-\frac{1}{3}\left(E_{1}+E_{2}\right)\left[\left(10 \beta_{10}+3 \beta_{5} H^{2}-\beta_{6} \mathcal{H}^{2}\right) \delta_{i j}\right. \\
+\left(10 \beta_{4}+\beta_{3}+3 \beta_{6}+2 \beta_{3} \mathcal{H}^{2}\right) \mathcal{H}_{i} \mathcal{H}_{j} \\
\left.+\left(10 \beta_{2}+5 \beta_{3}+3 \beta_{3} H^{2}\right) \varepsilon_{i j k} \mathcal{H}_{k}\right]
\end{gathered}
$$

onde os $\beta_{i}$ sâo obtidos da resoluçăo do sistema de equaçöes (4.169) a (4.178). O coeficiente de condutividade lérmica na ausência de campo magnético é dado pela equaçäo (4.138); ou seja

$$
A_{0}=\frac{3}{16} \frac{(\kappa+1)^{2}\left(2000 \kappa^{4}+9490 \kappa^{3}+13449 \kappa^{2}+7736 \kappa+1121\right)}{\left(1360 \kappa^{3}+3626 \kappa^{4}+3973 \kappa^{3}+2560 \kappa^{2}+969 \kappa+116\right)}
$$

Sem perda de generalídade, se considerarmos o carmpo magnético coincidente com a direça $x$ de um sistema de coordenadas cartesianas com origem fixa em algum ponto do gás temos, dà equaçào (4.18I):

$$
\left(\lambda^{(1)}\right)_{i j}=\left(\begin{array}{ccc}
\lambda_{x z}^{(1)} & 0 & 0 \\
0 & \lambda_{y y}^{(1)} & \lambda_{z z}^{(1)} \\
0 & -\lambda_{y \neq}^{(1)} & \lambda_{z z}^{(1)}
\end{array}\right)
$$

sendo

$$
\begin{gathered}
\lambda_{z z}^{(n)}=-\frac{1}{3}\left(E_{1}+E_{2}\right)\left[10 \beta_{10}+\left(10 \beta_{4}+4 \beta_{5}+2 \beta_{3}\right) H^{2}+2 \beta_{9} \mathcal{H}^{4}\right] \\
\lambda_{y y}^{(1)}=\lambda_{z:}^{(1)}=-\frac{1}{3}\left(E_{1}+E_{2}\right)\left(10 \beta_{10}+3 \beta_{5} \mathcal{H}^{2}-\beta_{5} \mathcal{H}^{2}\right) \\
\lambda_{y 2}^{(1)}=-\lambda_{z y}^{(1)}=-\frac{1}{3}\left(E_{1}+E_{2}\right)\left(10 \beta_{2}+5 \beta_{3}+3 \beta_{8} \mathcal{H}^{2}\right) \mathcal{K} .
\end{gathered}
$$

De acordo com as equações $(4.103)$ a (4.106) o tensor condutividade térmica dependente do campo magnético pode ser escrito como

$$
\left(\lambda^{(1)}\right)_{i j}=\left(\begin{array}{ccc}
\lambda_{11}^{n)} & 0 & 0 \\
0 & \lambda_{2}^{(1)} & \lambda_{i \tau}^{(1)} \\
0 & -\lambda_{i r}^{(1)} & \lambda_{\perp}^{(1)}
\end{array}\right)
$$


sendo que os coeficientes de condutividade térmica paralelo, perpendicular e transversal são dados, respectivamente, por

$$
\begin{aligned}
& \lambda_{l]}^{(1)}=\lambda_{x x}^{(1)}=-\frac{1}{3}\left(E_{1}+E_{2}\right)\left[10 \beta_{10}+\left(10 \beta_{4}+4 \beta_{5}+2 \beta_{6}\right) \mathcal{H}^{2}+2 \beta_{3} \mathcal{H}^{4}\right], \\
& \lambda_{ \pm}^{(1)}=\lambda_{z y}^{(1)}=\lambda_{z=}^{(1)}=-\frac{1}{3}\left(E_{1}+E_{2}\right)\left(10 \beta_{10}+3 \beta_{5} H^{2}-\beta_{5} H^{2}\right) \\
& \lambda_{L}^{(3)}=\lambda_{y z}^{[1}=-\lambda_{z y}^{(n)}=-\frac{1}{9}\left(E_{1}+E_{2}\right)\left(10 \beta_{2}+5 \beta_{3}+3 \beta_{8} \mathcal{H}^{2}\right) \mathcal{H}
\end{aligned}
$$

A variação relativa dos coeficientes de condutividade térmica paralelo e perpendicular tèm a forma da equacäo (4.108), ou seja

$$
\frac{\Delta \lambda_{i j}(K)}{\lambda_{i}}=\frac{\lambda_{i j}(H)-\lambda_{0} B_{i j}}{\lambda_{0}}=\frac{\lambda_{i j}^{(1)}(\mathcal{H})}{\lambda_{0}}
$$

onde $\lambda_{0}$ é dado pela equação (4.182).

Resolvendo o sistema de equacōes $(4.169)$ a $(4.178)$ para os coeficientes $p_{n}$, podemos escrever para a variação relativa do coeficiente de condutividade térmica paralelo (4.188)

$$
\frac{\Delta \lambda_{1}}{\lambda_{0}}=\frac{\lambda_{1}^{(1)}}{\lambda_{0}}=\frac{6\left(E_{1}+E_{2}\right)\left(D_{3}+2 D_{4}\right) D_{7}^{2} \beta_{1} H^{2}}{\lambda_{0}\left[\left(D_{3}+2 D_{4}\right)\left(D_{3}-D_{4}\right)\left(3 D_{3}-10 E+D_{4}\right)+D_{7}^{2}\left(3 D_{3}+4 D_{4}-4 E\right) H^{2}\right]}
$$

e para a variaçấo relativa do coeficiente de condutividade térmica perpendicular (4.189)

$$
\begin{aligned}
\frac{\Delta \lambda_{\perp}}{\lambda_{0}}=\frac{A_{L}^{\prime}}{\lambda_{0}}=\frac{-3 D_{3}^{2} \beta_{1}}{\delta \lambda_{0}}\left(E_{1}+E_{2}\right)\left[3\left(D_{4}-D_{3}\right)\left(D_{3}+2 D_{4}\right)^{2}\left(9 D_{3}-7 D_{4}-5 E\right)\right. \\
\left.-4 D_{7}^{2}\left(3 D_{3}+D_{4}\right)\left(3 D_{3}+D_{4}-E\right) H^{2}\right] H^{2}
\end{aligned}
$$

onde

$$
\begin{gathered}
\delta=\left(D_{3}+2 D_{4}\right)^{2}\left(D_{4}-D_{3}\right)^{2}\left(10 E-3 D_{3}-D_{4}\right)^{2}+ \\
D_{3}^{2}\left[45 D_{3}^{4}+102 D_{3}^{3} D_{4}-19 D_{3}^{2} D_{4}^{2}-100 D_{3} D_{4}^{3}+72 D_{4}^{4}-\right. \\
E\left(138 D_{3}^{3}+274 D_{3}^{2} D_{4}+160 D_{3} D_{4}^{2}-72 D_{4}^{3}\right)+ \\
\left.E^{2}\left(185 D_{3}^{2}+260 D_{3} D_{4}+180 D_{4}^{2}\right)\right] \mathcal{H}^{2}+4 D_{7}^{4}\left(E-3 D_{3}-D_{4}\right)^{2} H^{4}
\end{gathered}
$$

A variaçăo relativa para o coefecente de condutividade térmico transversal (4.190) é dada por 


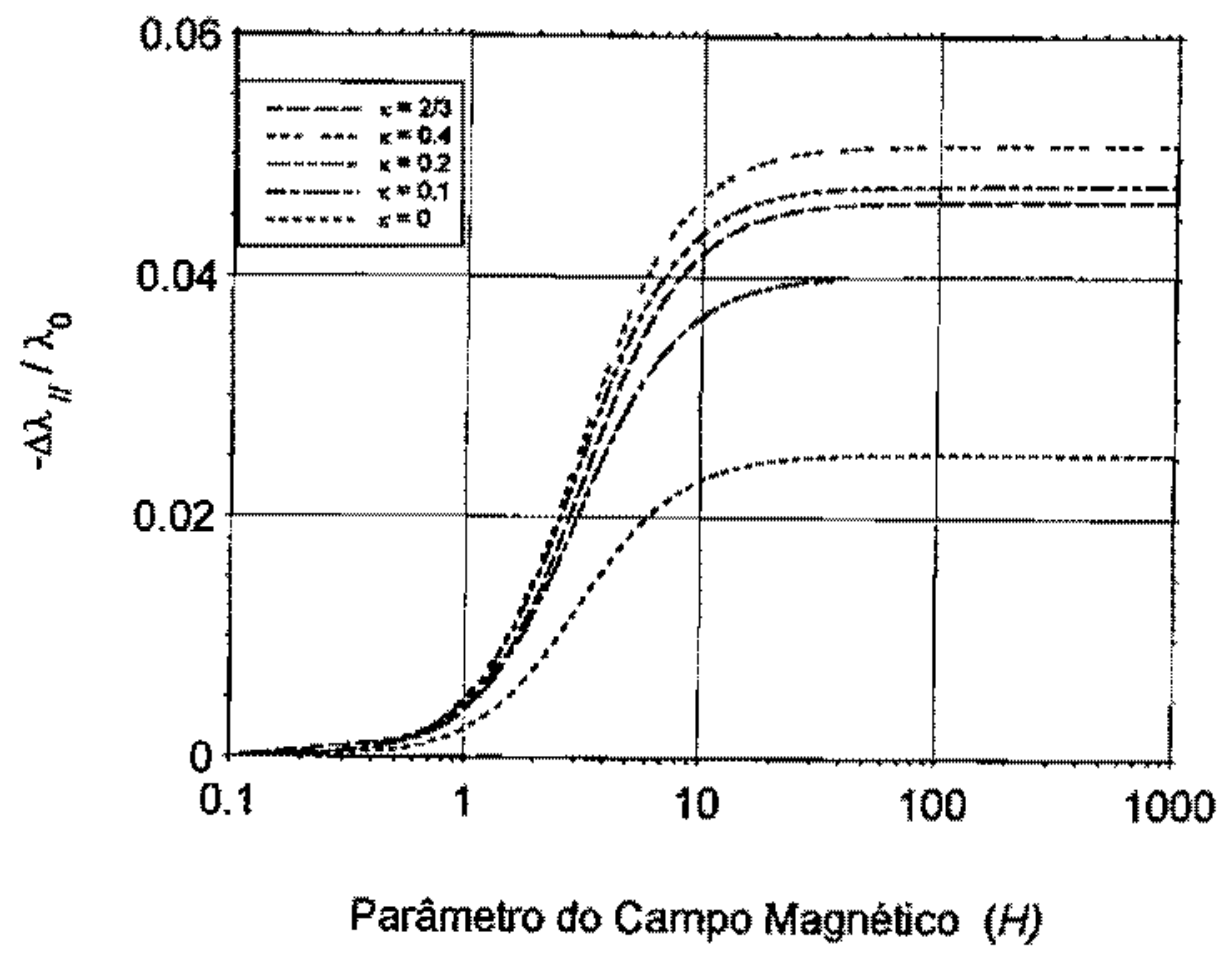

Figura 4.4: Variaçóes relativas para o coeficiente de condutividade têrmica paralelo.

$$
\begin{gathered}
\frac{\lambda_{\mathrm{tr}}}{\lambda_{0}}=\frac{\lambda_{t+}^{(1)}}{\lambda_{0}}=-\frac{3 \beta_{1} D_{7}}{\delta \lambda_{0}}\left(E_{1}+E_{2}\right)\left(3 D_{3}+D_{4}-10 E\right)\left[5\left(D_{4}-D_{3}\right)^{2}\left(D_{3}+2 D_{4}\right)^{2}+\right. \\
\left.4 D_{7}^{2}\left(2 D_{3}^{2}+2 D_{3} D_{4}+D_{4}^{2}\right) H^{2}\right] H
\end{gathered}
$$

Nas figuras $4.4,4.5$ e 4.6 são mostradas as variaçōes relativas das trếs componentes independentes do tensor condutividade térmica para alguns valores do momento de inếriatadimensional $n=\frac{4 I}{n t^{3}}$, segundo as equaçöes $(4.192),(4.193)$ e $(4.195)$. O paràmetro do campo é a quantidade

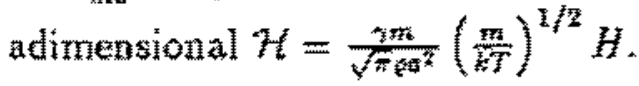

Estas curvas estäo em acordo com as observaçöes experimentais [62, 63], nas quais elas apre sentam um comportamento universal para todos os gases poliatômicos sob campo magnético aplicado, ou seja: i medida que a intensidade do campo magnético cresce os valores dos coeficiente de condutividade térmica $\lambda_{1}$ e $\lambda_{\|}$diminuem até atingirem um patamar que permanece constante; por outro lado, os valores do coeficiente $\lambda_{\mathrm{tr}}$ aumentam até atingirem um valor maximo apos o qual diminuem. No entanto, os valores absolutos de $\frac{\Delta \lambda}{\lambda_{0}}$ apresentados por este modelo sào ainda altos desde que os resultados experimentais mostram varjaçós relativas da ordem de 1 ou menores. Isto se deve a anomalia apresentada pelo modelo de esferas rugosas no qual mesmo em colisöes em que as esferas se tocam levemente elas são fortemente defletidas. Resultados teónicos semelhantes sã apresentados nas referências $[55,53]$. 


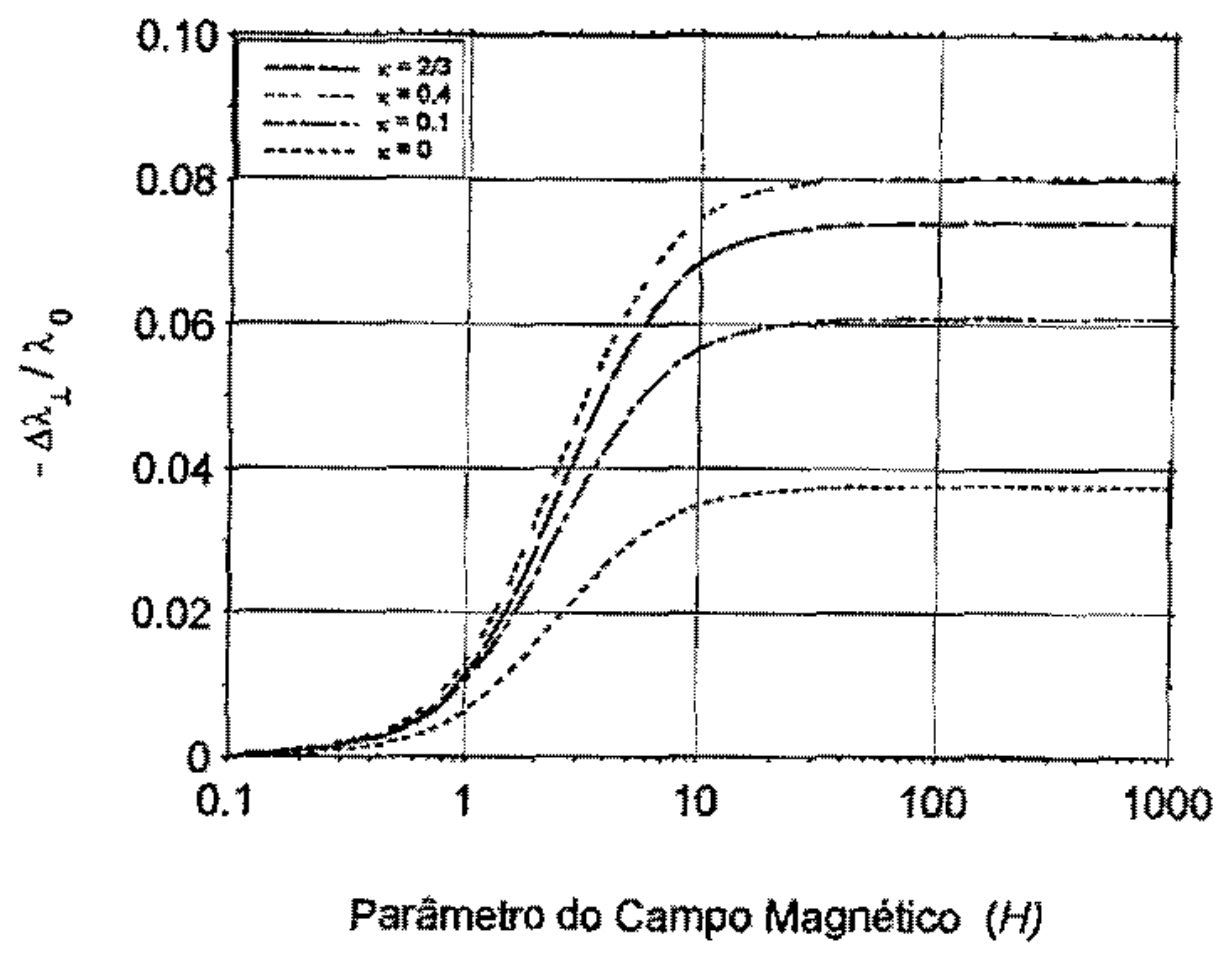

Figura 4.5: Variaçoes relativas para o coeficiente de condutividade de térmica perpendicular.

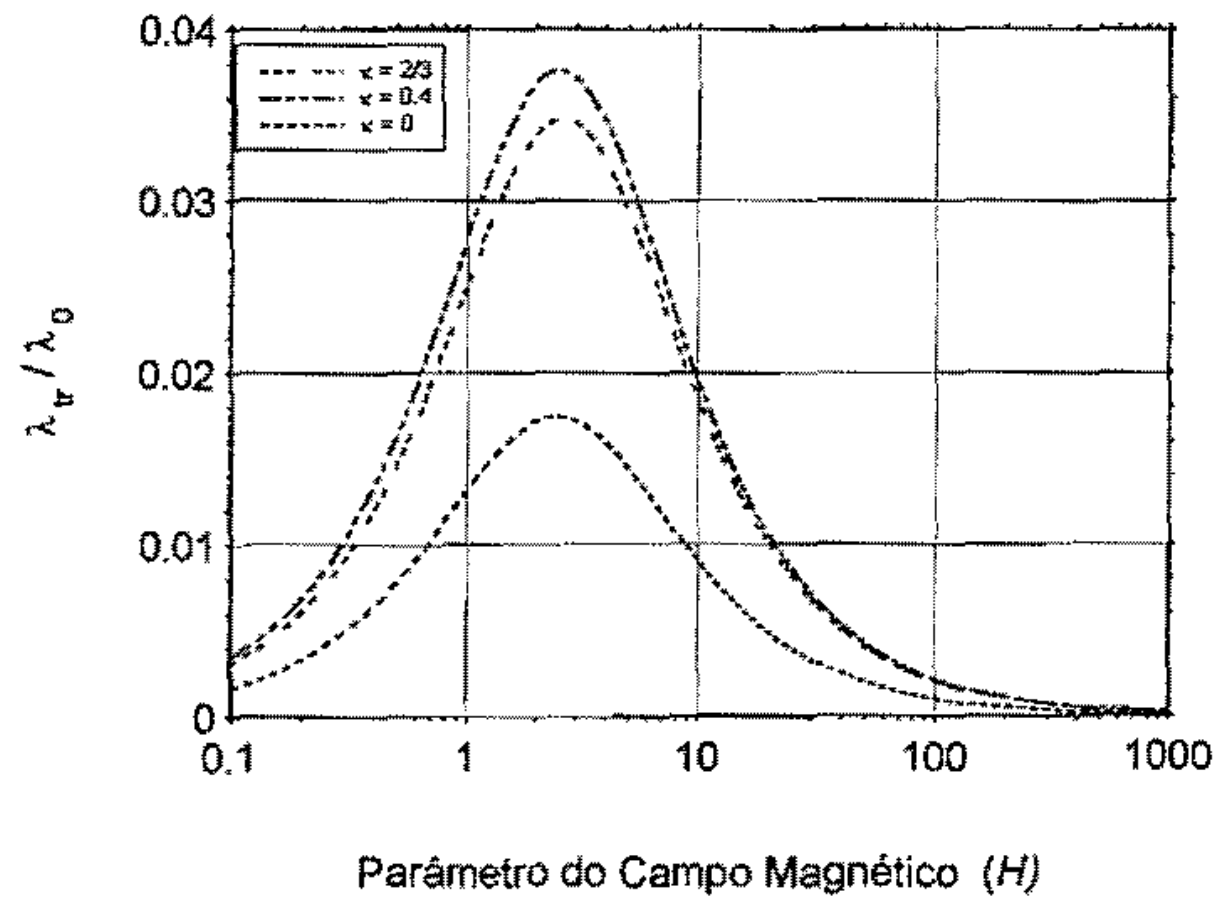

Figura 4.6: Variaços relativas para o coeficiente de condutividade tétmica transversal. 


\begin{tabular}{|c|c|c|c|}
\hline Gás & $\kappa$ & {$[R]_{\text {exp }}$} & {$[R]_{\text {teo }}$} \\
\hline$C H_{4}$ & 0,048 & 1,64 & 1,50 \\
$C D_{4}$ & 0.075 & 1,58 & 1,51 \\
$C F_{4}$ & 0.109 & 1,58 & 1,52 \\
\hline
\end{tabular}

Tabela 4. 1: Valores experimentais e teóricos para o parâmetro $R$.

Outro aspecto interessante está relacionado à grandeza $R=\left(\frac{\Delta \lambda_{1}}{\Delta \lambda_{1}}\right)_{\mathcal{K}_{-\infty}}$ ou seja, a relação entre a variaçấo relativa dos decréscimos dos coeficientes de condutividade térmica para os valores de saturaçẫo do campo magnético aplicado. Teoricamente, Kagan e Maksimov [51] obtiveram valor $3 / 2$ para qualquer gas poliatomico.

Para o modelo de esferas rugosas temos, das equações $(4.192)$ e (4.193):

$$
R=\left(\frac{\Delta \lambda_{1}}{\Delta \lambda_{\| l}}\right)_{K-\text { t }}=\frac{1}{2} \frac{\left(3 D_{3}+D_{4}\right)\left(3 D_{3}+4 D_{4}-4 E\right)}{\left(D_{3}+2 D_{4}\right)\left(3 D_{3}+D_{4}-E\right)}
$$

com $D_{3}$ e $D_{4}$ dados pela equaçäo (4.119) e $E$ dado pela equaçäo (4.156). $O$ gráfico da figura 4.7 mostra a varíą̧a de $R$ como funçẫo do momento de inércia adimensional $k=\frac{4 i}{m c^{2}}$. no intervalo $0 \leq \kappa \leq \frac{2}{3}$. Verificamos que $R$ varia desde o valor 1,475 para $\kappa=0$, que corresponde à concentraçăo de massa no centro da esfera, até o valor 1,600 para $\kappa=\frac{2}{3}$, que corresponde à distribuiçẫo uniforme de massa na sua superfície.

Na tabela 4.1 apresentamos valores obtidos experimentalmente para alguns gases poliatómicos [64] : os valores téricos foram calculados com a equação (4.196).

\subsection{Coeficientes de Viscosidade para Gases Poliatômicos}

Consideremos a determinaçăo dos coeficientes de viscosidade de um gás poliatômico sob um campo magnético aplicado $\mathbf{H}$, através do método combinado de Grad e Chapman. Enskog. Para isso, retomemos a equação (4.52)

$$
\begin{aligned}
& \int \varphi f^{(0)}\left\{\frac{m}{k T} C_{i} C_{j} \frac{\partial v_{\langle i}}{\partial x_{j\rangle}}+\frac{m}{6 k T}\left(C^{2}-\frac{I \Omega^{2}}{m}\right) \frac{\partial v_{r}}{\partial x_{r}}+\frac{1}{T}\left[\left(\frac{m C^{2}}{2 k T}-\frac{5}{2}\right)+\left(\frac{I \Omega^{2}}{2 k T}-\frac{3}{2}\right)\right] C_{i} \frac{\partial T}{\partial x_{i}}\right. \\
& \left.+\left[\frac{l}{m} \frac{q_{\langle p s\rangle}}{\varrho}\left(\frac{m}{h T}\right)^{2} n_{s}+\frac{h_{s}}{2 \ell}\left(\frac{m}{k T}\right)^{2} \varepsilon_{s p k} C_{k}+\frac{I}{m} \frac{h_{\langle p p\rangle k}}{\varrho}\left(\frac{m}{k T}\right)^{3} \Omega_{s} C_{k}\right] \gamma(\boldsymbol{\Omega} \times \mathbf{H})_{p}\right\} d \mathrm{~cd} d \omega \\
& =\int \phi I[\varphi] d c d \omega
\end{aligned}
$$

onde 


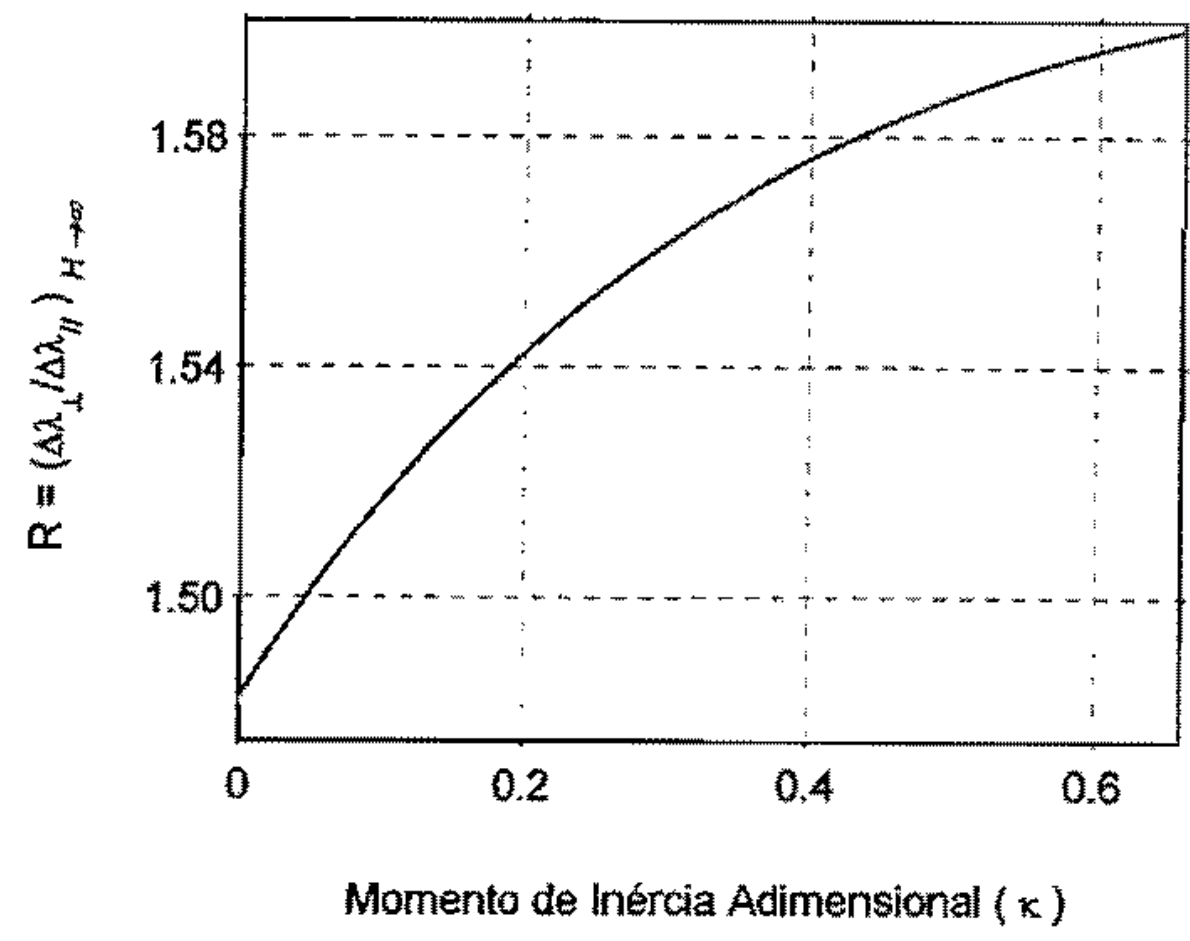

Figura 4.7: Variaçăo do parâmetro $R$ em funçăo do campo matgnético de saturaçăo.

$$
\mathcal{I}[\phi]=\int f_{1}^{(0)} f^{(0)}\left(\phi_{1}^{\prime}+\phi^{\prime}-\phi_{1}-\phi\right) a^{2}(\mathrm{~g} \cdot \mathbf{k}) d \mathbf{k} d \mathrm{c}_{1} d \omega_{1} d \mathrm{c} d \omega
$$

$\varphi(c, \omega, t)$ é uma funçâa arbitrătia e o desvio $\phi$ dado por

$$
\begin{aligned}
& \omega=\frac{1}{2 \varrho}\left(\frac{m}{k T}\right)^{2} m_{k i>} C_{i} C_{j}+\frac{1}{2 \varphi}\left(\frac{m}{k T}\right)^{2} \Pi\left(C^{2}-\frac{m n^{2}}{m}\right)+\frac{I}{2 m \rho}\left(\frac{m}{k T}\right)^{2} q_{\langle i j\rangle} \Omega_{i} \Omega_{j} \\
& +\frac{1}{\varrho}\left(\frac{m}{k T}\right)^{2}\left(\frac{m C^{2}}{5 k T}-1\right) q_{i}^{T} C_{i}+\frac{1}{\varrho}\left(\frac{m}{k T}\right)^{2}\left(\frac{I N^{2}}{3 k T}-1\right) q_{i}^{R} C_{i}+\frac{1}{2 Q}\left(\frac{m}{k T}\right)^{2} h_{i} \varepsilon_{i j} \Omega_{j} C_{k} \\
& +\frac{1}{2 m p}\left(\frac{m}{h T}\right)^{3} h_{<i j>k} \Omega_{i} n_{3} C_{k}
\end{aligned}
$$

Sob campo magnético externo aplicado o gás torna-se anisotrópico de modo que o tensor pressão é escrito como

$$
p_{i j}=\left(p-\eta_{v} \frac{\partial v_{t}}{\partial x_{r}}\right) b_{i j}-2 \eta_{<i j><k t>} \frac{\partial v_{<k}}{\partial x_{l\rangle}}
$$

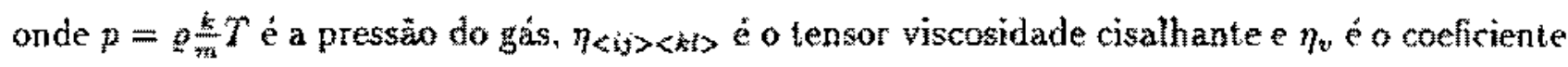
de viscosidade volumétrica. Nosso objetivo é, através da equacäo (4,197), obter relaçoes entre oa campos $p_{i j}$ e $\frac{\partial_{a}}{a x}$ de modo a determinar os coeficientes de viscosidade cisaltante volumétrica como funços do campo magnético aplicado. 


\subsubsection{Determinação dos Coeficientes de Viscosidade Cisalhante}

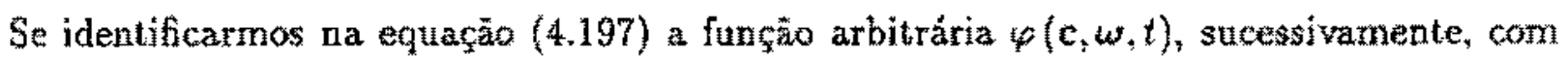

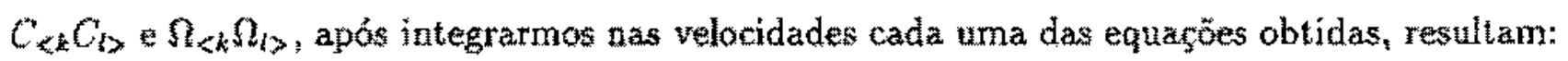

$$
\begin{aligned}
& \frac{2 g k T}{m^{2}} \frac{\partial v_{<k}}{\partial x_{i}}=-\frac{8 \sqrt{\pi}}{15} \rho\left(\frac{a}{m}\right)^{2}\left(\frac{k T}{m}\right)^{1 / 2} \frac{1}{(n+1)^{2}}\left[(13 \kappa+6) p_{<k t>}+5 \kappa q_{<k t>}\right],
\end{aligned}
$$

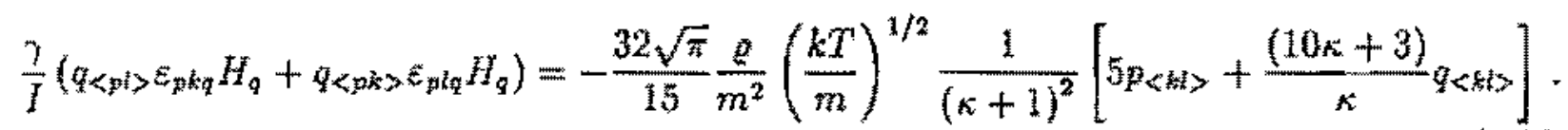

Introduzindo o campo magnético adimensional dado pela equaçă (4.63)

$$
\mathcal{H}_{i}=\frac{\eta m}{g a^{2}}\left(\frac{m}{\eta k T}\right)^{1 / 2} H_{i}
$$

as equajỏes (4.201) e (4.202) podem ser escritas como (veja apêndice B)

$$
\begin{aligned}
& \frac{\partial v_{<i}}{\partial x_{3>}}=-\frac{4 a^{2}}{15 m}\left(\frac{\pi m}{k T}\right)^{1 / 2} \frac{1}{(\kappa+1)^{2}}\left[(13 \kappa+6) p_{\langle i j\rangle}+5 \kappa q\langle i j\rangle\right] \\
& q<j k>\varepsilon_{k i q u} H_{q}+q_{k i k\rangle} \varepsilon_{k i \eta} H_{q}+\frac{8 \kappa}{15(\kappa+1)^{2}}\left[p_{k\langle i j\rangle}+\frac{(10 \kappa+3)}{\kappa} q_{\langle i j\rangle}\right]=0
\end{aligned}
$$

que constituem um sistema de equaçöes tensoriaj para determinaço dos campos $p<j\rangle$ e $q<i>$. Procuraremos obter uma equação na forma $p_{\langle j\rangle}=-n_{\langle i j\rangle<k b} \frac{\partial u_{<k}}{\partial z_{i\rangle}}$, a fim de obter os coeffcientes de viscosidade cisalhante para o gás.

As componentes de um tensor de quarta ordem, sem traço nos dois primeiros e ûtimos indices e que depende do vetor axial campo magnetico $\mathcal{H}_{i}$ podem ser escritas como (veja apèndice $\mathrm{B}$ )

$$
\begin{aligned}
& A_{\langle i j\rangle\langle k\rangle\rangle}=a_{1}\left(\delta_{j k \varepsilon_{i q q}}+\delta_{i k} \epsilon_{l j Q}+\delta_{j t \varepsilon_{k q}}+\delta_{i j k_{k q q}}\right) \mathcal{H}_{q} \\
& +b_{k}\left(\delta_{i k} \delta_{j !}+\delta_{i k} \delta_{j k}-\frac{2}{3} \delta_{i j} \delta_{k l}\right)
\end{aligned}
$$

onde $a_{1}$ e $b_{1}$ sâo coeficientes a determinar. Assim, podemos escrever que

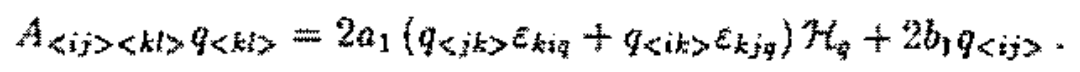

As equaçōes $(4.204)$ e $(4.205)$ podem ser escritas como 


$$
\frac{\partial v_{\langle i}}{\partial x_{j\rangle}}=\alpha_{1} p_{\langle i j\rangle}+\alpha_{2} q_{\langle i j\rangle}
$$

e

$$
A<i j\rangle\langle k|>\langle<k i\rangle+\alpha_{3} p_{<i j\rangle}=0
$$

onde introduzimos os parämetros

$$
\begin{gathered}
\alpha_{1}=-\frac{4 a^{2}}{15 m}\left(\frac{m m}{k T}\right)^{1 / 2} \frac{13 \kappa+6}{(\kappa+1)^{2}} \\
\alpha_{2}=-\frac{4 a^{2}}{3 m}\left(\frac{\pi m}{k T}\right)^{1 / 2} \frac{\kappa}{(\kappa+1)^{2}} \\
\alpha_{3}=\frac{16}{3} \frac{\kappa}{(\kappa+1)^{2}} \\
\alpha_{4}=b_{1}=\frac{8}{15} \frac{10 \kappa+3}{(k+1)^{2}}
\end{gathered}
$$

com $a_{y}=1$. Desta forma, podemos reescrever a equaçäo $(4.206)$ como

$$
\begin{aligned}
A\langle i j\rangle\langle k i\rangle & =\left(\delta_{i k_{i k q}}+\delta_{i k} \varepsilon_{i q}+\delta_{j \varepsilon_{k i q}}+\delta_{i \mid \varepsilon_{k i q}}\right) H_{q} \\
& +\alpha_{4}\left(\delta_{i k} \delta_{j i}+\delta_{i j} \delta_{j k}-\frac{2}{3} \delta_{i j} \delta_{k j}\right)
\end{aligned}
$$

Da equaçào $(4.209)$ temos

$$
p_{\langle i j\rangle}=-\frac{1}{a_{3}} A_{\langle i j\rangle\rangle\langle k l\rangle} q\langle k b\rangle
$$

e substituindo esta equação na (4.208) resulta:

$$
\frac{\partial v_{\langle i}}{\partial x_{j\rangle}}=-\frac{\alpha_{1}}{\alpha_{j}} A_{\langle i j\rangle\langle k|>} q_{\langle k \mid\rangle}+\alpha_{2} q_{\langle i j\rangle} .
$$

A equaça anterior pode ser escrita na forma

$$
\left.\frac{\partial v_{<i}}{\partial x_{j\rangle}}=A_{<i j\rangle\langle k i>}^{\prime} q<k \neq\right\rangle
$$

onde int roduzimos o tensor

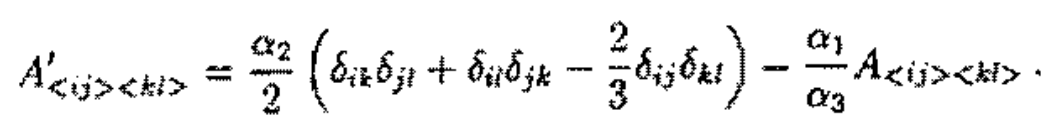


Considerando que (veja apêndice $\mathrm{B}$ )

$$
\left(A^{\prime-1}\right)_{\langle m n>\langle i\rangle\rangle} A_{\langle i j\rangle\langle k i\rangle}^{\prime}=\frac{1}{2}\left(\delta_{m k} \delta_{n !}+\delta_{m k} \delta_{n k}-\frac{1}{3} \delta_{m n} \delta_{k t}\right)
$$

obtemos, da equacta $(4.217)$ :

$$
q\langle k l\rangle=\left(A^{\prime-1}\right)_{\langle k i>\langle i j\rangle} \frac{\partial v_{<i}}{\partial x_{j\rangle}}
$$

onde

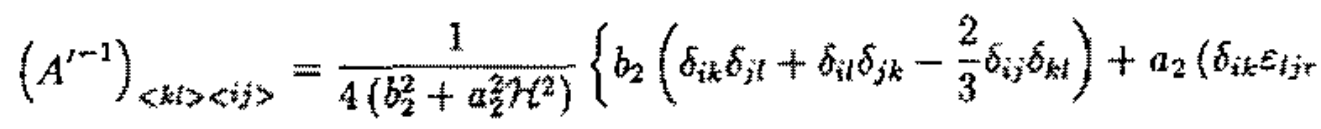

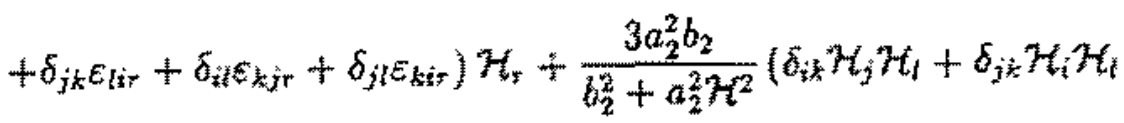

$$
\begin{aligned}
& \left.+\delta_{i} H_{j} H_{k}+\delta_{j l} \mathcal{H}_{i} \mathcal{H}_{k}-\frac{4}{3} H_{j} \mathcal{H}_{k} \mathcal{H}_{i}-\frac{4}{3} \delta_{h i} H_{i} \mathcal{H}_{j}+\frac{4}{9} H^{2} \delta_{k l} \delta_{i j}\right) \\
& \pm \frac{3 a_{2}^{3}}{b_{2}^{2}+a_{2}^{2} \mathcal{H}^{2}}\left(\varepsilon_{k i} \mathcal{H}_{j} \mathcal{H}_{l}+\varepsilon_{k j} H_{i} \mathcal{H}_{i}+\varepsilon_{i r} \mathcal{H}_{j} \mathcal{H}_{k}+\varepsilon_{i j r} \mathcal{H}_{i} \mathcal{H}_{k}\right) \mathcal{H}_{*}
\end{aligned}
$$

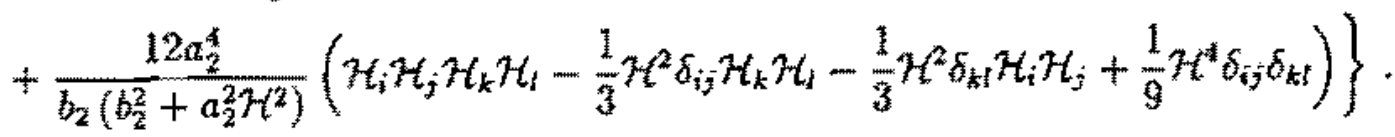

Na equaçã̃o anterior introduzimos

$$
a_{2}=-\frac{\alpha_{1}}{\alpha_{3}}
$$

e

$$
b_{2}=\frac{\alpha_{2}}{2}-\frac{\alpha_{1} \alpha_{4}}{\alpha_{3}}
$$

Substituindo as equaçôes $(4.214),(4.220)$ e (4.221) na $(4.215)$ obtemos para o deviante do tensor pressãa:

$$
\begin{aligned}
& p_{\langle i j\rangle}=-\frac{1}{2 \alpha_{3}\left(b_{2}^{2}+4 a_{2}^{2} h^{2}\right)}\left\{\left(b_{2} \alpha_{i}+4 a_{2} h^{2}\right)\left(\delta_{i k} \delta_{j t}+\delta_{i j} \delta_{j k}-\frac{2}{3} \delta_{i j} \delta_{k l}\right)\right. \\
& +\frac{1}{2}\left(b_{2}-a_{2} \alpha_{4}\right)\left(\delta_{i l} \varepsilon_{k j q}+\delta_{i k \varepsilon_{i q q}}+\delta_{j t \varepsilon_{k i q}}+\delta_{j k \varepsilon_{i q}}\right) \mathcal{H}_{q}
\end{aligned}
$$

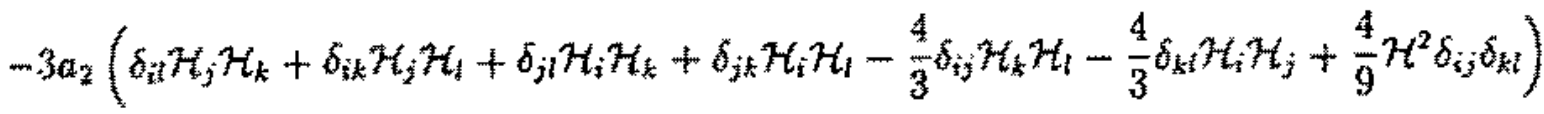

$$
\begin{aligned}
& +\frac{3 a_{2}^{2} b_{2}}{b_{2}^{2}+a_{2}^{2} \mathcal{H}^{2}}\left[\alpha _ { i } \left(\delta_{i 1} \mathcal{H}_{j} \mathcal{H}_{k}+\delta_{i k} \mathcal{H}_{j} \mathcal{H}_{i}+\delta_{j l} \mathcal{H}_{i} \mathcal{H}_{k}+\delta_{j k} \mathcal{H}_{i} \mathcal{H}_{i}-\frac{4}{3} \delta_{i j} \mathcal{H}_{k} \mathcal{H}_{j}-\frac{4}{3} \hat{k}_{k} \mathcal{H}_{i} \mathcal{H}_{2}\right.\right.
\end{aligned}
$$

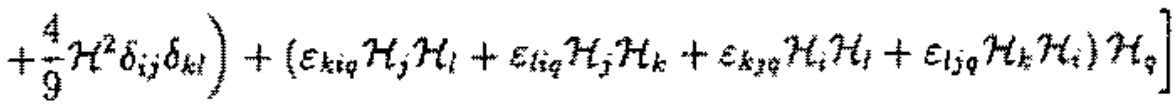




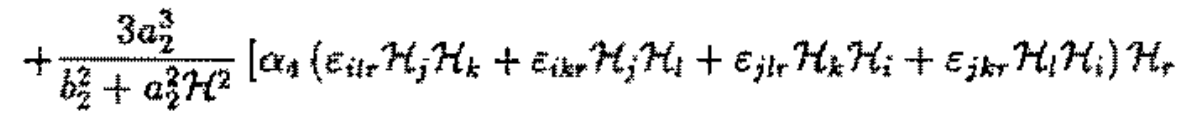

$$
\begin{aligned}
& \left.+H^{2} \delta_{i l} H_{j} H_{k}+H^{2} \delta_{i k} H_{i} H_{j}+H^{2} \hat{\delta}_{j} \mathcal{H}_{i} H_{k}+H^{2} \delta_{j k} \mathcal{H}_{i} H_{1}-4 H_{i} \mathcal{H}_{j} H_{k} H_{1}\right] \\
& \left.+\frac{12 a_{2}^{4} \alpha_{4}}{b_{2}\left(b_{2}^{2}+a_{2}^{2} \mathcal{H}^{2}\right)} H_{<i} H_{j} H_{<k} H_{l>}\right\} \frac{\partial v_{<k}}{\partial x_{b}} .
\end{aligned}
$$

Por outro lado, da equaçấ $(4.200)$ temos para o deviante do tensor pressăto

$$
p_{\langle i j\rangle}=-2 \pi\langle i\rangle\langle k \xi\rangle \frac{\partial v_{\langle k}}{\partial x_{l\rangle}}
$$

de modo que as componentes do tensor viscosidade cisalhante da equaçu (4.224), säo dadas por

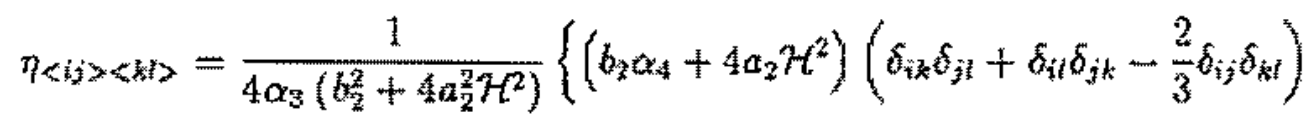

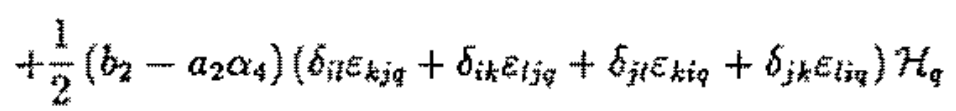

$$
\begin{aligned}
& -3 a_{2}\left(\delta_{i j} H_{j} H_{3}+\delta_{i k} \mathcal{H}_{j} H_{i}+\delta_{j} H_{i} H_{k}+\delta_{j k} H_{i} H_{i}-\frac{4}{3} \delta_{i j} H_{k} H_{t}-\frac{4}{3} \delta_{k l} H_{i} H_{j}+\frac{4}{9} H^{2} \delta_{i j} \delta_{k l}\right)
\end{aligned}
$$

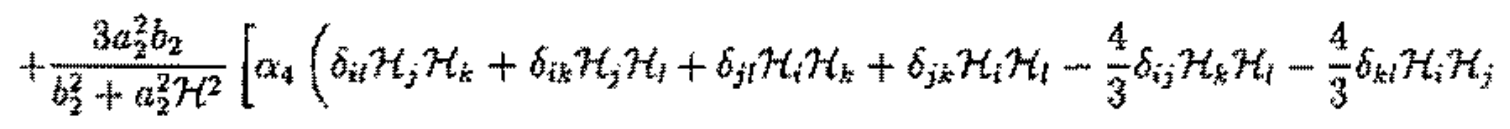

$$
\begin{aligned}
& \left.\left.+\frac{4}{9} H^{2} \delta_{i j} \delta_{k l}\right)+\left(\varepsilon_{k i q} H_{j} H_{l}+\varepsilon_{k q} H_{j} H_{k}+\varepsilon_{k j q} \mathcal{H}_{i} H_{i}+\varepsilon_{i j q} \mathcal{H}_{k} \mathcal{H}_{i}\right) H_{i}\right]
\end{aligned}
$$

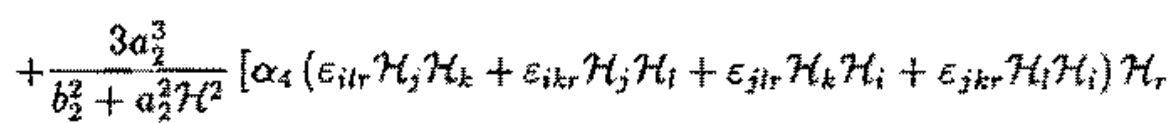

$$
\begin{aligned}
& \left.+\mathcal{H}^{2} \delta_{i} \mathcal{H}_{j} \mathcal{H}_{k}+\mathcal{H}^{2} \delta_{3 k} \mathcal{H}_{i} \mathcal{H}_{j}+\mathcal{H}^{2} \delta_{j 1} \mathcal{H}_{i} H_{k}+\mathcal{H}^{2} \delta_{j k} \mathcal{H}_{i} H_{i}-4 \mathcal{H}_{i} \mathcal{H}_{j} \mathcal{H}_{k} \mathcal{H}_{i}\right] \\
& \left.+\frac{12 a_{2}^{4} \alpha_{i}}{b_{2}\left(b_{2}^{2}+a_{2}^{2} H^{2}\right)} H_{<i} \mathcal{H}_{j>} \mathcal{H}_{<\mathrm{k}} \mathcal{H}_{\rangle}\right\}
\end{aligned}
$$

Com objetivo de explicitar as componentes do tensor viscosidade cisalhante, consideremos o campo magnético orientado segundo o eixo $x$ de um sistema cartesiano de coordenadas com origem fixa ern algum ponto do gás. Se considerarmos que o traço do deviante do tensor gradiente de velocidade $e^{\prime}$ nulo, isto

$$
\frac{\partial v_{<i}}{\partial x_{j>}}=\frac{\partial v_{<x}}{\partial x_{z>}}+\frac{\partial v_{<\psi}}{\partial x_{y>}}+\frac{\partial v_{<z}}{\partial x_{z>}}=0
$$

podemos consiruir as componentes do tensor p<ijs: através das equaçoes (4.225) a (4.227): é o que faremos em seguida. 
- componente $p_{\langle x+\rangle}$

Da equação (4.225) temos

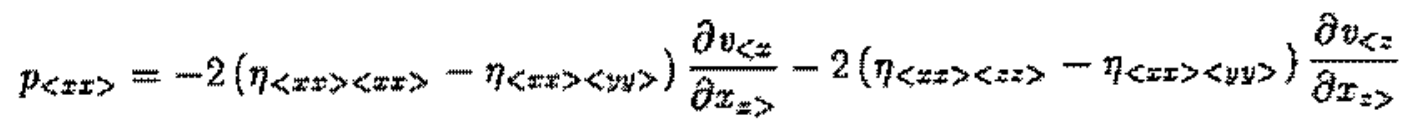

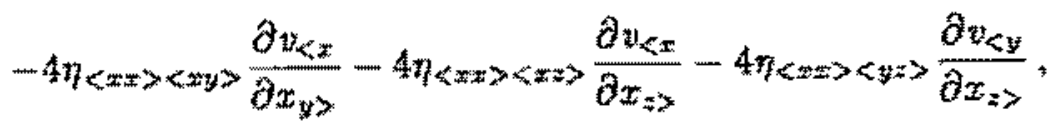

ou

$$
\left.p_{\langle x\rangle}=-2(n\langle x\rangle\langle x\rangle-n)\langle x\rangle\langle y\rangle\right) \frac{\partial v_{\langle x}}{\partial x_{z>}}
$$

sendo

$$
\begin{aligned}
& \eta_{\langle \pm x><x z>}=\frac{1}{3 \alpha_{3}\left(b_{2}^{2}+4 a_{2}^{2} h^{2}\right)}\left[b_{2} \alpha_{4}+\frac{4 a_{2}^{2} b_{2} \alpha_{4}}{b_{2}^{2}+a_{2}^{2} h^{2}} h^{2}+\frac{4 a_{2}^{4} \alpha_{4}}{b_{2}\left(b_{2}^{2}+a_{2}^{2} H^{2}\right)} h^{4}\right] \\
& n_{\langle x x\rangle\langle y y\rangle}=n_{\langle \pm x\rangle\langle z\rangle\rangle}= \\
& \frac{-1}{6 \alpha_{3}\left(b_{2}^{2}+4 a_{2}^{2} \mathcal{H}^{2}\right)}\left[b_{2} \alpha_{4}+\frac{4 a_{2}^{2} b_{2} \alpha_{4}}{b_{2}^{2}+a_{2}^{2} \mathcal{H}^{2}} K^{2}+\frac{4 a_{2}^{4} \alpha_{4}}{b_{2}\left(b_{2}^{2}+a_{2}^{2} \mathcal{H}^{2}\right)} \mathcal{H}^{4}\right]
\end{aligned}
$$

e

$$
\eta_{\langle x x\rangle\langle x y\rangle}=\eta_{\langle x x\rangle\langle x z\rangle}=\eta_{\langle x x\rangle\langle y z\rangle}=0 .
$$

A equaçäo (4.229) pode então ser reescrita como

$$
p_{\langle x x\rangle}=-2 \eta_{1} \frac{\partial \eta_{\langle x}}{\partial x_{x>}}
$$

onde

$$
\begin{aligned}
\eta_{1} & =\eta_{\langle x z>\langle x z>}-\eta_{\langle x x>\langle x y\rangle} \\
& =\frac{1}{2 \alpha_{3}\left(b_{2}^{2}+4 a_{2}^{2} \gamma^{2}\right)}\left[b_{2} \alpha_{4}+\frac{4 a_{2}^{2} b_{2} \alpha_{4}}{b_{2}^{2}+a_{2}^{2} \mu^{2}} H^{2}+\frac{4 a_{2}^{4} \alpha_{4}}{b_{2}\left(b_{3}^{2}+a_{2}^{2} H^{2}\right)} H^{4}\right] \\
& =\frac{a_{4}}{2 b_{2} \alpha_{3}}
\end{aligned}
$$


- componente $p_{\langle y y\rangle}$

$$
\begin{gathered}
p_{\langle y y\rangle}=-2\left(\eta_{\langle y y\rangle\langle y y\rangle}-\eta_{\langle y y\rangle\langle x x>}\right) \frac{\partial v_{\langle y}}{\left.\partial x_{y}\right\rangle}-2\left(\eta_{\langle y y\rangle\langle z z>}-\eta_{\langle y y\rangle\langle x x>}\right) \frac{\partial v_{<z}}{\left.\partial x_{z}\right\rangle} \\
-4 \eta_{\langle y y\rangle\langle y z>} \frac{\partial v_{\langle y}}{\partial x_{z>}},
\end{gathered}
$$

onde

$$
\begin{aligned}
& \eta_{<y y><y y>}=\frac{1}{3 \alpha_{3}\left(b_{2}^{2}+4 a_{2}^{2} \mathcal{H}^{2}\right)}\left[b_{2} \alpha_{4}+\left(3 a_{2}+\frac{a_{2}^{2} b_{2} \alpha_{4}}{b_{2}^{2}+a_{2}^{2} \mathcal{H}^{2}}\right) \mathcal{H}^{2}+\frac{a_{2}^{4} \alpha_{4}}{b_{2}\left(b_{2}^{2}+a_{2}^{2} \mathcal{H}^{2}\right)} \mathcal{H}^{4}\right] \\
& \left.\eta_{\langle y y\rangle}\right\rangle\langle x x\rangle=-\frac{1}{6 \alpha_{3}\left(b_{2}^{2}+4 a_{2}^{2} \mathcal{H}^{2}\right)}\left[b_{2} \alpha_{4}+\frac{4 a_{2}^{2} b_{2} \alpha_{4}}{b_{2}^{2}+a_{2}^{2} \mathcal{H}^{2}} \mathcal{H}^{2}+\frac{4 a_{2}^{4} \alpha_{4}}{b_{2}\left(b_{2}^{2}+a_{2}^{2} \mathcal{H}^{2}\right)} \mathcal{H}^{4}\right] \\
& \eta_{<y y><z z>}=-\frac{1}{6 \alpha_{3}\left(b_{2}^{2}+4 a_{2}^{2} \mathcal{H}^{2}\right)}\left[b_{2} \alpha_{4}+\left(6 a_{2}-\frac{2 a_{2}^{2} b_{2} \alpha_{4}}{b_{2}^{2}+a_{2}^{2} \mathcal{H}^{2}}\right) \mathcal{H}^{2}-\frac{2 a_{2}^{4} \alpha_{4}}{b_{2}\left(b_{2}^{2}+a_{2}^{2} \mathcal{H}^{2}\right)} \mathcal{H}^{4}\right], \\
& \eta_{\langle y y\rangle\langle x y\rangle}=\eta_{\langle y y\rangle\langle x z\rangle}=0, \\
& \eta_{\langle y y>\langle y z>}=\frac{\left(a_{2} \alpha_{4}-b_{2}\right) \mathcal{H}}{2 \alpha_{3}\left(b_{2}^{2}+4 a_{2}^{2} \mathcal{H}^{2}\right)} .
\end{aligned}
$$

Reescrevemos a equação (4.238) como

$$
p_{\langle y y>}=-2 \eta_{2} \frac{\partial v_{<y}}{\partial x_{y>}}-2 \eta_{4} \frac{\partial v_{<y}}{\partial x_{z>}}-2\left(\eta_{1}-\eta_{2}\right) \frac{\partial v_{<z}}{\partial x_{z>}},
$$

onde definimos

$$
\begin{aligned}
\eta_{2}= & \eta_{\langle y y\rangle\langle y y\rangle}-\eta_{\langle y y\rangle\langle x x\rangle}= \\
& \frac{1}{2 \alpha_{3}\left(b_{2}^{2}+4 a_{2}^{2} \mathcal{H}^{2}\right)}\left[b_{2} \alpha_{4}+\left(2 a_{2}+\frac{2 a_{2}^{2} b_{2} \alpha_{4}}{b_{2}^{2}+a_{2}^{2} \mathcal{H}^{2}}\right) \mathcal{H}^{2}+\frac{2 a_{2}^{4} \alpha_{4}}{b_{2}\left(b_{2}^{2}+a_{2}^{2} \mathcal{H}^{2}\right)} \mathcal{H}^{4}\right] 4
\end{aligned}
$$


e

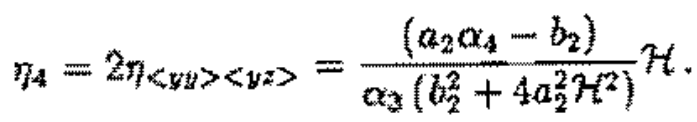

- componente $p_{<z \pm>}$

$$
\begin{aligned}
& p_{\langle i z\rangle}=-2\left(\eta_{\langle z z\rangle\langle y y\rangle}-\eta_{\langle z z\rangle\langle z z\rangle}\right) \frac{\partial v_{\langle y}}{\partial w_{y\rangle}}-2\left(\eta_{\langle z z\rangle\langle z z\rangle}-\eta_{\langle z z\rangle\langle x z\rangle}\right) \frac{\partial v_{\langle x}}{\partial v_{z\rangle}} \\
& \rightarrow 4 \eta_{\langle; z><y z>} \frac{\partial v_{<y}}{\partial x_{m>}}
\end{aligned}
$$

onde

$$
\begin{aligned}
& \eta_{\langle z z>\langle x y\rangle}=\eta\langle z z>\langle x z\rangle=0 \\
& \left.\eta_{\langle z z}\right\rangle\langle y y\rangle= \\
& -\frac{1}{6 \alpha_{3}\left(b_{3}^{3}+4 a_{2}^{2} \mathcal{H}^{2}\right)}\left[b_{3} \alpha_{4}+6 a_{2} \mathcal{H}^{2}-\frac{2 a_{2}^{2} b_{2} \alpha_{4}}{b_{3}^{2}+a_{2}^{2} \mathcal{H}^{2}} \mathcal{H}^{2}-\frac{2 a_{2}^{4} \alpha_{4}}{b_{2}\left(b_{2}^{2}+a_{2}^{2} \mathcal{H}^{2}\right)} \mathcal{H}^{4}\right] \\
& \eta\langle z z\rangle\langle x x\rangle= \\
& -\frac{1}{6 \alpha_{3}\left(b_{2}^{2}+4 a_{2}^{2} \mathcal{H}^{2}\right)}\left[b_{2} \alpha_{4}+\frac{4 a_{2}^{2} b_{2} \alpha_{4}}{b_{2}^{2}+a_{2}^{2} h^{2}} \mathcal{H}^{2}+\frac{4 a_{2}^{*} \alpha_{4}}{b_{2}\left(b_{3}^{2}+a_{2}^{2} \mathcal{H}^{2}\right)} \mathcal{H}^{4}\right] \\
& \eta_{\langle z x\rangle\langle x \#\rangle}= \\
& \frac{1}{3 \alpha_{3}\left(b_{2}^{2}+4 a_{2}^{2} \mathcal{H}^{2}\right)}\left[b_{2} \alpha_{4}+\left(3 a_{2}+\frac{a_{2}^{2} b_{2} \alpha_{4}}{b_{3}^{2}+a_{2}^{2} H^{2}}\right) \mathcal{H}^{2}+\frac{a_{2}^{4} \alpha_{4}}{b_{2}\left(b_{2}^{2}+a_{2}^{2} \mathcal{H}^{2}\right)} H^{4}\right]
\end{aligned}
$$

e

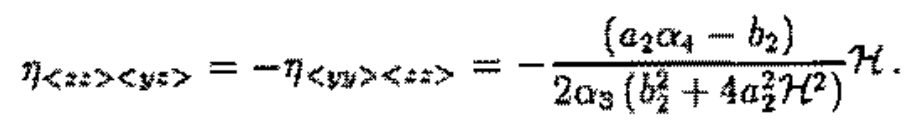

Reescreverno a equação $(4.248)$ como

$$
p_{<\#>>}=-2\left(\eta_{1}-\eta_{4}\right) \frac{\partial v_{\langle y}}{\partial x_{y\rangle}}-2 \eta_{2} \frac{\partial v_{<x}}{\partial x_{z>>}}+2 \eta_{4} \frac{\partial v_{<y}}{\partial x_{z>}}
$$


onde

$$
\begin{gathered}
\eta_{1}-\eta_{2}=\eta_{\langle z z>\langle y y\rangle}-\eta_{\langle z z>\langle x x\rangle}= \\
\frac{a_{2}}{\alpha_{3}\left(b_{2}^{2}+4 a_{2}^{2} \mathcal{H}^{2}\right)}\left[\frac{a_{2} b_{2} \alpha_{4}}{b_{2}^{2}+a_{2}^{2} \mathcal{H}^{2}}+\frac{a_{2}^{3} \alpha_{4}}{b_{2}\left(b_{2}^{2}+a_{2}^{2} \mathcal{H}^{2}\right)}-1\right] \mathcal{H}^{2} \\
\eta_{2}=\eta_{\langle z z>\langle z z\rangle}-\eta_{\langle z z>\langle x x\rangle}=\eta_{\langle y y\rangle\langle y y\rangle}-\eta_{\langle y y\rangle\langle x x\rangle}= \\
\frac{1}{2 \alpha_{3}\left(b_{2}^{2}+4 a_{2}^{2} \mathcal{H}^{2}\right)}\left[b_{2} \alpha_{4}+\left(2 a_{2}+\frac{2 a_{2}^{2} b_{2} \alpha_{4}}{b_{2}^{2}+a_{2}^{2} \mathcal{H}^{2}}\right) \mathcal{H}^{2}+\frac{2 a_{2}^{4} \alpha_{4}}{b_{2}\left(b_{2}^{2}+a_{2}^{2} \mathcal{H}^{2}\right)} \mathcal{H}^{4}\right] \\
\eta_{4}=-2 \eta_{\langle z z>\langle y z>}=\eta_{\langle y y\rangle\langle y z>}=\frac{\left(a_{2} \alpha_{4}-b_{2}\right)}{\alpha_{3}\left(b_{2}^{2}+4 a_{2}^{2} \mathcal{H}^{2}\right)} \mathcal{H} .
\end{gathered}
$$

e

- componente $p_{\leq y z>}$

$$
p_{\langle y z>}=-4 \eta_{\langle y z>\langle y z>} \frac{\partial v_{<y}}{\partial x_{z\rangle}}-2 \eta_{\langle y z>\langle y y\rangle} \frac{\partial v_{\langle y}}{\partial x_{y\rangle}}-2 \eta_{\langle y z>\langle z z>} \frac{\partial v_{<z}}{\partial x_{i>}} .
$$

Esta equação pode ser reescrita como

$$
p_{<y z>}=-2\left(2 \eta_{2}-\eta_{1}\right) \frac{\partial v_{<y}}{\partial x_{z>}}+\eta_{4} \frac{\partial v_{<y}}{\partial x_{y>}}-\eta_{4} \frac{\partial v_{<z}}{\partial x_{z>}}
$$

sendo

$$
\begin{gathered}
\eta_{\langle y z><x y\rangle}=\eta_{\langle y z>\langle x z\rangle}=\eta_{\langle y z>\langle x x\rangle}=0, \\
2 \eta_{2}-\eta_{1}=2 \eta_{\langle y z>\langle y z>}=\frac{b_{2} \alpha_{4}+4 a_{2} \mathcal{H}^{2}}{2 \alpha_{3}\left(b_{2}^{2}+4 a_{2}^{2} \mathcal{H}^{2}\right)}, \\
\eta_{4}=-2 \eta_{<y z><y y\rangle}=2 \eta_{<y z><z z>}=\frac{\left(a_{2} \alpha_{4}-b_{2}\right)}{\alpha_{3}\left(b_{2}^{2}+4 a_{2}^{2} \mathcal{H}^{2}\right)} \mathcal{H} .
\end{gathered}
$$

- componente $p_{\langle x z\rangle}$

$$
p_{<x=>}=-2 \eta_{5} \frac{\partial v_{<x}}{\partial x_{x>}}-2 \eta_{3} \frac{\partial v_{<x}}{\partial x_{z>}}
$$


sendo

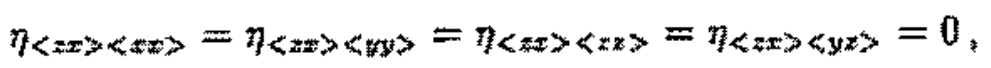

$$
\begin{aligned}
& \eta_{k}=2 \eta_{<x><y>}=\frac{\left(b_{2}-a_{2} \alpha_{4}\right)}{2 \alpha_{3}\left(b_{3}^{2}+4 a_{2}^{2} \mathcal{H}^{2}\right)}\left(1+\frac{3 a_{2}^{2} H^{2}}{b_{2}^{2}+a_{2}^{2} \mathcal{H}^{2}}\right) \mathcal{H}
\end{aligned}
$$

e

$$
\begin{aligned}
& \eta_{3}=2 \eta\langle z z\rangle\langle x z\rangle= \\
& \frac{1}{2 \alpha_{3}\left(b_{2}^{z}+a_{2}^{2} \mathcal{H}^{2}\right)}\left[b_{2} \alpha_{4}+a_{2}\left(1+\frac{3 a_{2} b_{2} \alpha_{4}}{b_{2}^{2}+a_{2}^{2} h^{2}}\right) \mathcal{H}^{2}+\frac{3 a_{2}^{3}}{b_{2}^{2}+a_{3}^{3} \mathcal{H}^{2}} \mathcal{H}^{4}\right]
\end{aligned}
$$

- componente p<xy

$$
p_{<x y>}=-2 \eta_{3} \frac{\partial v_{<x}}{\partial x_{y>}}+2 \eta \frac{\partial z_{<x}}{\partial x_{z>}}
$$

sendo

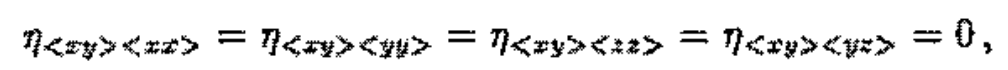

e

$$
\begin{aligned}
& \eta_{3}=2 \eta_{\langle x y\rangle\langle y\rangle}=2 \eta_{<x \neq><x z>}= \\
& \frac{1}{2 \alpha_{3}\left(b_{2}^{2}+4 a_{2}^{2} H^{2}\right)}\left[b_{2} \alpha_{4}+a_{2}\left(1+\frac{3 a_{2} b_{2} \alpha_{4}}{b_{2}^{2}+a_{2}^{2} H^{2}}\right) H^{3}+\frac{3 a_{2}^{3}}{b_{2}^{2}+a_{2}^{2} M^{2}} K^{4}\right]
\end{aligned}
$$

e

$$
\eta_{5}=-2 \eta_{\langle x y\rangle\langle x ;\rangle}=\eta_{\langle x x\rangle\langle x y\rangle}=\frac{\left(b_{2}-a_{2} \alpha_{4}\right)}{2 a_{3}\left(b_{2}^{2}+4 a_{2}^{2} \mathcal{H}^{2}\right)}\left(1+\frac{3 a_{2}^{2} \mathcal{H}^{2}}{b_{2}^{2}+a_{2}^{2} \mathcal{H}^{2}}\right) \mathcal{H}
$$

Estas relaçoes entre as componentes do deviante do tensor pressäo as respectivas componentes do deviante do gradiente de velocidade 5 aso as mesmas encontradas em [60], obtidas através de consideraçōes de simetria e das relaçöes de Onsager. Elas säo mostradas na tabela 4.2 onde as linhas e colunas conectam, respectivarnente, fuxos e forças termodinatmicas.

Para referencia posterior relacionamos em seguida os cinco coeficientes independentes de viscosidade cisalhante:

$$
\eta_{1}=\frac{\alpha_{4}}{2 b_{2} \alpha_{3}}
$$




\begin{tabular}{|c|c|c|c|c|c|c|}
\hline & $\frac{\partial v \times x}{\partial E x z}$ & $\frac{\partial w_{0}}{\partial z_{0}}$ & 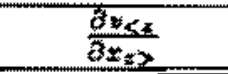 & $\frac{\partial \varepsilon_{x y}}{\partial z_{2} z}$ & $\frac{3+4 k x}{\left.\partial x_{x}\right\rangle}$ & $\frac{\partial z_{k x}}{\partial z_{v 2}}$ \\
\hline$p<x$ & $-2 \eta_{1}$ & $\overline{0}$ & 0 & 0 & 0 & 0 \\
\hline$p<y y\rangle$ & 0 & $-2 \eta_{2}$ & $-2\left(7_{1}-7_{2}\right)$ & $-2 \eta_{4}$ & 0 & 0 \\
\hline$p\langle q z\rangle$ & 0 & $-2\left(\eta_{1}-\eta_{2}\right)$ & $-2 \eta_{2}$ & $2 \eta_{4}$ & 0 & 0 \\
\hline$p<y z>$ & 0 & $\eta_{4}$ & $-\eta_{4}$ & $2 \pi_{1}-4 m_{2}$ & 0 & 0 \\
\hline$p_{\langle z \#\rangle}$ & 0 & 0 & 0 & 0 & $-27 / 3$ & $-2 \eta_{5}$ \\
\hline$p_{<x y+y}$ & 0 & 0 & 0 & 0 & $27_{5}$ & $-2 n_{3}$ \\
\hline
\end{tabular}

Tabela 4.2: Componentes do tensor pressäo

$$
\begin{gathered}
\eta_{2}=\frac{1}{2 \alpha_{3}\left(b_{2}^{2}+4 a_{2}^{2} \mathcal{H}^{2}\right)}\left[b_{2} \alpha_{4}+\left(2 a_{2}+\frac{2 a_{2}^{2} b_{2} \alpha_{1}}{b_{2}^{2}+a_{2}^{2} \mathcal{H}^{2}}\right) \mathcal{H}^{2}+\frac{2 a_{2}^{4} \alpha_{4}}{b_{2}\left(b_{2}^{2}+a_{2}^{2} \mathcal{H}^{2}\right)} \mathcal{H}^{4}\right] \\
\eta_{3}=\frac{1}{2 \alpha_{3}\left(b_{2}^{2}+4 a_{2}^{2} \mathcal{H}^{2}\right)}\left[b_{2} \alpha_{4}+a_{2}\left(1+\frac{3 a_{2}^{2} b_{2} \alpha_{4}}{b_{2}^{2}+a_{3}^{2} \mathcal{H}^{2}}\right) \mathcal{H}^{2}+\frac{3 a_{2}^{3}}{b_{2}+a_{2}^{2} \mathcal{H}^{2}} \mathcal{H}^{4}\right] \\
\eta_{4}=\frac{\left(a_{2} \alpha_{4}-b_{2}\right)}{\alpha_{3}\left(b_{2}^{2}+4 a_{2}^{2} \mathcal{H}^{2}\right)} \mathcal{H} \\
\eta_{5}=\frac{\left(b_{2}-a_{2} \alpha_{4}\right) \mathcal{H}}{2 \alpha_{3}\left(b_{2}^{2}+4 a_{2}^{2} \mathcal{H}^{2}\right)}\left(1+\frac{3 a_{2}^{2}}{b_{2}^{2}+a_{2}^{2} \mathcal{H}^{2}}\right) \mathcal{H}
\end{gathered}
$$

com os pazametros $\alpha_{2}, \alpha_{3}, \alpha_{4}, a_{2}$ e $b_{2}$ dados pelas equaçóes $(4.211),(4.212),(4.213),(4.222)$ e $(4.223)$, respectivamente. Observemos que os coeficientes $\eta_{2}$ e $\eta_{3}$ sào funçốs pares enquanto que $\eta_{4}$ 诘 sâo funçố impares do campo magnético $H$; neste modelo o coeficiente $\eta_{1}$ independe do campo magnético aplitcado. Na figura 4.8 apresentamos as variaçôs relativas para os coeficientes de viscosidade cisalhante dados pelas equaçōes (4.274) a (4.278). Estes resultados concordam de forma satisfatória com os apresentados na referência [06].

$\mathrm{Na}$ ausència de campo magnético $(M=0)$, o gás torna-se isotrópico e obtemos das equaçóes $(4.274)$ a $(4.278)$ :

$$
\eta_{1}=\eta_{2}=\eta_{3}=\eta_{0}=\frac{\alpha_{4}}{2 b_{2} \alpha_{3}}
$$

$$
\eta_{4}=\eta_{5}=0
$$

Assim, o deviante do tensor pressão da equação (4.225) se reduz a

$$
p_{\langle i \xi\rangle}=-2 \eta_{0} \frac{\partial v_{\langle i}}{\partial x_{i\rangle}}
$$




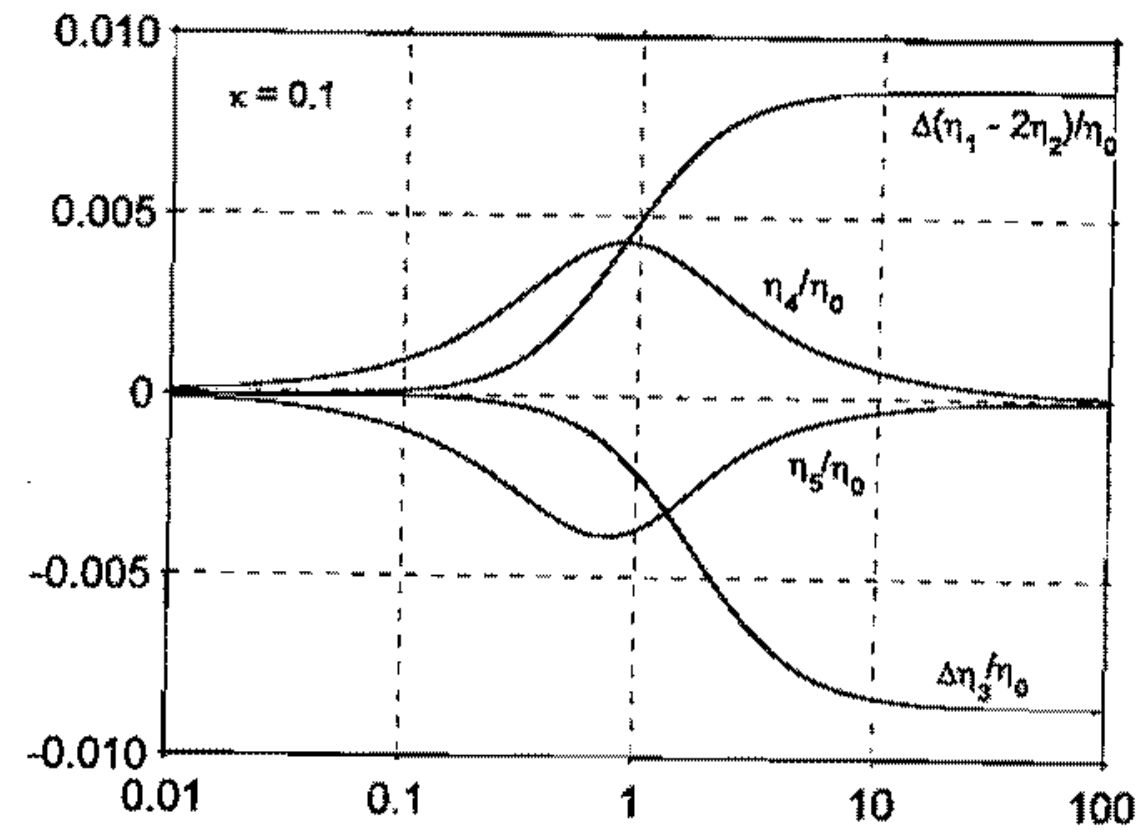

Parâmetro do Campo Magnético (H)

Figura 4.8: Variaçoes relativas dos coeficientes de viscosidade cisalhante.

com o coeficiente de viscosidade cisalbante dado por

$$
n_{\mathrm{o}}=\frac{5}{8 a^{2}}\left(\frac{m k T}{\pi}\right)^{3 / 2} \frac{(n+1)^{2}(10 \kappa+3)}{\left(35 k^{2}+33 \kappa+6\right)} .
$$

Este resultado foi obtido anteriormente por Condiff, Lu e Dahler [61].

É interessante notar que se tornarmos o campo $q\langle i\rangle>0$, porianto uma aproximaçăo menos precisa para o cálculo dos coeficientes de viscosidade, obtemos da equaçào (4.201) uma equaçằo análoga a (4.281), com o coeficiente de viscosidade cisalhante dado por

$$
\eta_{0}=\frac{15}{8 a^{2}}\left(\frac{m k T}{\pi}\right)^{3 / 2} \frac{(k+1)^{2}}{(13 \kappa+6)}
$$

que é o resultado obtido por Pidduck [39],(9].

Com a finalidade de caracterizarmos melhor os cinco coeficientes independentes de viscosidade cisalhante consideremos alguns aspectos de suas medidas.

A equaçào de movimento para o gás é obtida através das equaçōes $(2.13)$ e $(2.14)$ com $F_{i}=0$. isto é

$$
\varrho \frac{\partial v_{i}}{\partial t}+\varrho v_{i} \frac{\partial u_{i}}{\partial x_{j}}+\frac{\partial p_{i j}}{\partial x_{j}}=0
$$




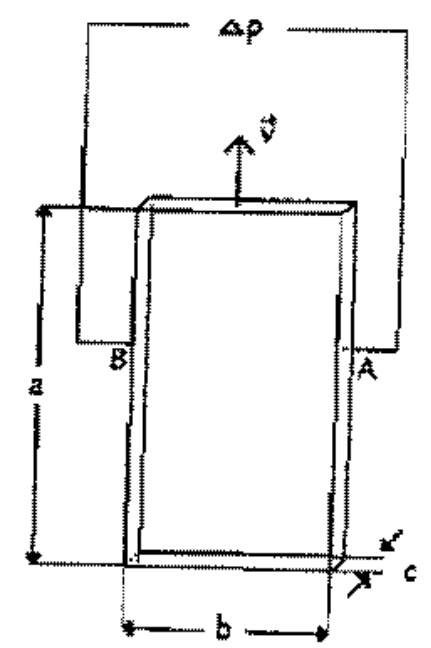

Figura 4.9: Dispositivo empregado na medida dos coeficientes de viscosidade.

e com o tensor pressão dado pela equação (4,200), temos

$$
\frac{\partial v_{i}}{\partial t}+p v_{3} \frac{\partial v_{i}}{\partial x_{j}}+\frac{\partial p}{\partial x_{i}}-\eta_{v} \frac{\partial^{*} p_{i}}{\partial x_{j} \partial x_{r}}-2 \eta_{\langle i j\rangle\langle k\rangle} \frac{\partial^{2} v_{<k}}{\partial x_{j} \partial x_{1\rangle}}=0
$$

A análise da forma completa da equaçäo (4.285) é complexa, de modo que é necessário um arramo experimental para o qual poucas derivadas de segunda ordem da velocidade sejam importantes. O arranjo mais simples empregado é o capilar rétangular [16] mostrado na figura 4.9. Para um capilar com dimensöes tais que

$$
a \geqslant b>c
$$

somente a componente da velocidade do gás a longo do capilar ( $v_{z}$ ) é importante bem como apenas o gradiente da velocidade na direção $y$, isto é, ( $\left.\frac{g_{s}}{z_{y}}\right)$, é relevante.

Com o campo magnético aplicado segundo alguma orientaçăo relativa ao capilar; dois efeitos experimentais säo observados:

(i) eleitos longitudinats, nos quais ocorrem mudanças na resistência ao fuxo do găs. Neste caso, os coeficientes de viscosidade longitudinats envolvidos säo $\eta_{1}, \eta_{2}$ e $\eta_{3}$; experimentalmente são medidos $\eta_{3}$ e a combinaçäo $2 \eta_{2}-\eta_{1}[50]$. As medidas säo feitas mantendo-se, por exemplo, a taxa de fluxo constante a longo do capilar;

(ii) afeitos transversais. nos quais gradientes de pressão transversais ao fluxo sâo medidos: os coeficientes de viscosidade transversais envolvidos șäo $\eta_{4}$ e $\eta_{x}$. Os gradjentes de pressào são medidos através da conexảo de um manômetro nos pontos $\mathrm{A}$ e $\mathrm{B}$, mostrados na figura 4.9 .

Orientando-se o campo magnético em diferentes posiçóes relalivas ao capilar é possivel 


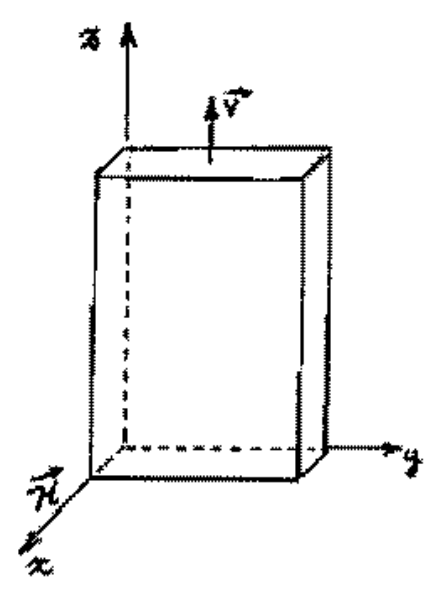

Figura 4.10: Arranjo esquernático utilizado na determinaça dos coeficientes de viscasidade $\eta_{3}$ e 政.

medir os cinco coeficientes independentes de viscosidade. Consideremos, como ilustraçãa, a determinação dos coeficientes de viscosidade longitudinal $\eta_{3}$ e transversal $\eta_{5}$. Da equação (4.285) com $\eta_{v}=0$ e no regine de fuxo estacionário, temos

$$
\varrho v_{j} \frac{\partial v_{i}}{\partial x_{j}}+\frac{\partial p}{\partial x_{i}}=2 \eta_{\langle i j\rangle\langle k i\rangle} \frac{\partial^{2} v_{\langle k}}{\partial x_{j} \partial x_{l\rangle}} .
$$

Na figura 4.10 é o mostrado o fluxo do gás na direçăo a com o campo magnético aplicado

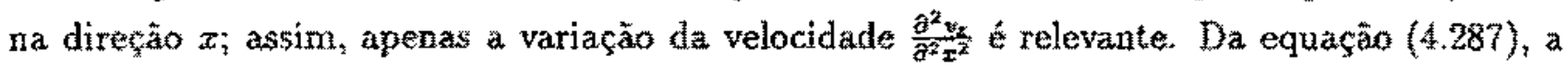
diferença de pressäo longitudinal é dada por

$$
\frac{\partial p}{\partial z}=2 \eta_{\langle x x\rangle\langle x z\rangle} \frac{\partial^{2} v_{<x}}{\left.\partial x^{2}\right\rangle}
$$

Com a equaçẫo (4.268) podemos escrever a equação anterior como

$$
\frac{\partial p}{\partial z}=\eta_{3} \frac{\partial^{2} v_{<z}}{\partial x^{2}>}
$$

Assim, mantendo-se constante o fluxo do gás ao longo do capilar (isto é, determinando $\frac{\partial p}{\partial z}$ ) e conhecendo o perfil de velocidades de fluxo do gás, o coeficiente de viscosidade $\eta_{3}$ está determintado.

Na determinação do coeficiente de viscosidade transversal $\eta_{\mathfrak{s}}$ a diferença de pressảo entre os pontos A e B ể medida (veja figura 4.9). Da equação (4.287) temos:

$$
\frac{\partial p}{\partial y}=2 \eta_{\langle x y\rangle<x z>} \frac{\partial^{2} v_{<=}}{\left.\partial x^{2}\right\rangle}
$$


e, comparando com a equação $(4,273)$ podemos berever que

$$
\frac{\partial p}{\partial y}=-n \frac{\partial^{2} v_{<z}}{\partial x^{2}>}
$$

de modo que o coeficiente $\eta_{s}$ pode ser determinado. A determinaçầ dos demais coeficientes de viscosidade cisalhante segue procedimentos análogos [65].

\subsubsection{Coeficiente de Viscosidade Volumétrica}

Para obtermos o coeficiente de viscosidade volumétrica para o gás identificamos a funçào arbitrária $\varphi(x, t)$ na equaçẫo $(4.197) \operatorname{com}\left(C^{2}-\frac{m n^{2}}{m}\right) \Pi$ e, após realizarmos as integraçōes da equaçăo obtida, resulta para a presșẫo dinămica:

$$
\Pi=-\eta_{v} \frac{\partial v_{i}}{\partial x_{i}}
$$

corn o coeficiente de viscosidade volumétrica dado por

$$
\eta_{v}=\frac{1}{32 a^{2}}\left(\frac{m k T}{\pi}\right)^{1 / 2} \frac{(\kappa+1)^{2}}{\kappa}
$$

que é o resultado obtido anteriormente por Pidduck [39] [9]. Desta forma a inclusão na teoria da $\Omega_{<i} \Omega_{j>}$ näo é suficiente para que tenhamos a dependência da do coeficiente $\eta_{t}$ com o campo magnético.

\subsection{Conclusồes}

O método de Chapman-Enskog e o método de Crad são os métodos tradicionalmente em. pregados na resoluçăo da equação de Boltzmann. Recentemente Bezera Jr, Reinecke Kremer [18] desenvolverarn uma forma alternativa que combina curacteristicas de ambos os métodos, denominado método combinado de Chapman-Enskog Grad. Com o método combinado toram obtidos os coeficientes de transporte para gases monoatömicos e suas misturas [18] bem como para gases ionizados [19], com bons resultados; além disso, houve uma considerável reduçấo no processo de cálculo quando comparado aos métodos tradicionais.

Nosso objetivo neste trabalho foi analisar as variaçoses dos coeficientes de transporte para um gás poliatônico sob o feito de un campo magnético externo uniforme e estacionário (efeito Senflleben-Beenakker), através do método combinado de Chapman-Enskog e Grad. Para isso empregamos un modelo em que consideramos as noléculas como esferas perfeitamente rígidas. elásticas e rugosas (modelo de Bryan). Neste modelo, a velocidade relativa dos pontos das esferas que entratn em contato sầ revertidos após uma colisăo binária. 
No método combinado a funçäo de distribuição para o gús num estado próximo ao do equilibrio e a lunçấo de distribuiçăo de Grad. Para obte-la empregamos um procedimento que consiste na maximizaçào da densidade de entropia cujos vínculos são os campos básicos definidos pelas equaçoes (4.8) a (4.17). Este é um problema típico do cálculo variacional e para resolve-lo empregamos o método dos multiplicadores de Lagrange.

Tendo deterrninado a funçäo de distribuiçäo de Grad para o problema o próximo passo do método combinado consiste em substitui-la na equaçăo de Boltzmann, como no método de Chapman-Enskog. Após a linezarizaçäo da equação obtida e a substituiçào das derivadas temporais através das equaçōes de balanço de um fluido em equilibrio (fluido de Euler) obtivemos uma equaç̃̃o semelhante à equaçăo integral que ocorte quando empregamos o método de Chapman-Enskog [veja equaçâo (4.52)]. O passo seguinte na aplicação do método consiste em multiplicar esta equação por funções adequadas nas velocidades linear angular e integra-las nestas velocidades; assim, obternos relaçỏes entre as forças e os fluxos termodinấmicos com coeficientes que estão relacionados aos coeficientes de transporte. Vamos analisar em seguida os principais resultados obticlos para os coeficientes de condutividade térmica, viscosidade cisalhante viscosidade volumétrica para um gás poliatómico.

\section{Coeficientes de Condutividade Térmica}

E conhecido da literatura específica que a polarização $\Omega_{<i} \Omega_{j>} C_{k}$ é que mais fortemente infuencia os coeficientes de condutividade térmica para um gás poliatónico enquanto que a polarizaça $(\Omega \times)_{i}=\varepsilon_{i j} \Omega_{j} C_{n}$ pode apresentar efeitos menores [50].

Introduzimos na teoria a polarizaça $\varepsilon_{i j k} \rho_{j} C_{k}$ (também denominado vetor de Waldmann) através do campo básico $h_{i}(x, h)$ definido pela equaçäo $(4.16)$. Na seçâ 4.5 .2 verificamos que o coeficiente de condutividade térmica paralelo $\lambda_{\text {倠 }}$ não ế afetado pelo campo campo magnético, $\phi$ que é incompativel com as observaçöes experimentais. Por outro lado, o coeficiente de condutividade perpendicular $\lambda_{1}$ apresenta um pequeno aumento relativo at $\dot{e}$ atíngir um patarnar para campos magnéticos de saturaço (veja figura 4.2); o coeficiente de condutividade transversal $\lambda_{*}$ decresce até atingir um valor minimo após o qual retorna aos valores iniciais, para grandes valores do campo magnético aplicado. Estes dados concordam com os obtíos por Dahler [53] para o modelo de esferas rugosas. Observamos, no entanto, que a inclusăo do campo $h_{i}\left(x_{*} t\right)$ na teoria provoca um aumento no coeficiente de condutividade térmica perpendicular e uma diminuição relativa no coeficiente de condutividade térmica transversal. Estes comportamentos opostos aos observados experimentalmente indicam que o campo $h_{i}(x, t)$ pode ser considerado como una contribuiçäo secundária à teoria.

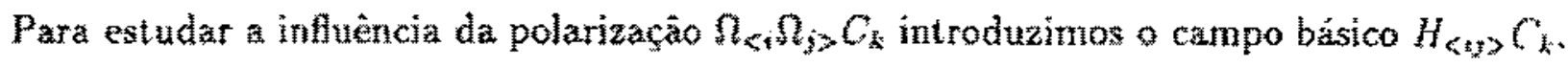


definido pela equaçầ (4.113). Como conseqüência obtivemos o sistema de equaçöes (4.114) a (4.116) o qual envolve relações entre os fluxos de calor rotacional $Q_{i}^{R}$ e translacional $Q_{i}^{T}$, o gradiente de temperatura $\frac{\partial T}{\partial x_{i}}$ e próprio campo básico $H_{\langle i j>k}$. A obtenção da solução deste sistema de vinte e uma equaçoes para o mesmo número de incógnitas apresenta alguma diffculdade, desda que näo é possivel resolve-lo algebricamente para obter relaçōes entre o fluxo total te calor $Q_{i}=Q_{i}^{T}+Q_{i}^{R}$, o tensor $H_{<i j>k}$ e o gradiente de temperatura $\frac{\partial T}{\partial r_{1}}$. Assim, é necessário expandir $H_{<i j\rangle}$ em termos do gradiente de temperatura e do campo magnético. Na literatura especifca encontramos que expansôes semelhantes a esta săo realizadas nem sempre com clareza requerida. Com o objetivo de exprimir o teasor $H_{\langle i j\rangle k}$ em termos do gradiente de lemperatura e do campo magnético, ampregamos teoremas de pepresentacta [21] os quais nos fornecem uma forma sistemática de representaçâ, inclusive para outras polarizaçōes que vierem a ser incorporadas à teoria (veja apêndice C).

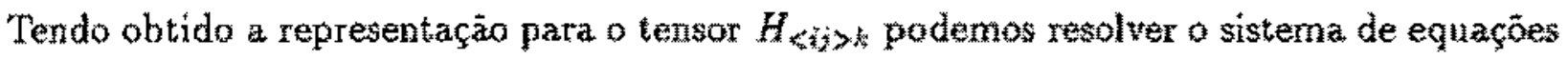
(4.114) a (4.116) e determinar expressões para as variaço è relativas dos tres coeficientes de condutividade térmica, isto é, para os coeficientes de condutividade térmica paralelo $\frac{\Delta \lambda_{1}}{\lambda_{0}}$ [equaça (4.192)], perpendicular $\frac{\Delta \lambda_{1}}{\lambda_{0}}$ [equação (4.193)] e transversal $\frac{\lambda_{x}}{\lambda_{0}}$ [ equaçāo (4.195)]. Nas figuras 4.4, 4.5 e 4.6 săo apresentados os gráficos para estas variaçồ relativas em termos do parâmetro de campo magnético $\mathcal{H}=\frac{\gamma m}{e^{2^{2}}}\left(\frac{m}{\pi k T}\right)^{1 / 2} H$. Verificamos que eles apresentam as principais ca. vacterísticas obtidas experimentalmente, isto $\hat{e}_{2}$ os coeficientes $\lambda_{\|}$e $\lambda_{\perp}$ apresentam pequenas reduçôes até atingirem um patamar constante para campos magnéticos de saturação enquanto que o coeficiente $\lambda_{t r}$ exibe um valor máximo após o qual tende para o valor nulo para campos magnéticos de saturação.

Outro aspecto a ressaltar se refere à razäo $R$ da equação (4.196) a qual relaciona as variaçoses $\Delta \lambda_{\| l}$ e $\Delta \lambda_{1}$ para campos de saturacão. Kagan Maksimov [bl] mostraram que $R=3 / 2$ para todos os gases poliatômicos; na tabela 4.1 apresentumos os valores de $R$ obtidos experimen. talmente para alguns gases polatömicos. O grafico da figura 4.7, obtido da equaçü (4.196). mostra a variação do parämetro $R$ para diferentes valores do parâmetro moleculat $n=\frac{4 !}{\text { ana }^{2}}$.

\section{Coeficientes de Viscosidade Cisalhante}

$O$ coeficiente de viscosidade cisalhante para um gás poliatômico submetido a um campo magnético externo deixä de ser um escalar ( $\left.\eta_{0}\right)$ e passa a ser representado pelo tensor de quarta ordem $\eta_{<i j\rangle\langle k l}$, o qual apresenta vinte e cinco componentes. Destas, apenas cinco säo independentes: três componentes longitudinais $\left(\eta_{1}, \eta_{2}\right.$ e $\left.\eta_{3}\right)$ relacionadas ao fuxo do gás ao longo de um tubo capilar e duas componentes transversais $\left(\eta_{4}\right.$ e $\left.\eta_{5}\right)$ relacionadas às direcoes mutuamente perpendiculares a tuxo do gás e à direçăo do campo magnético aplicado (veja 
seça 0.6 .1$)$

$\mathrm{Na}$ determinação dos coeficientes de viscosidade cisalhante $\Omega_{<} \Omega_{j>}$ é a a polarizaçäo dominante [49] de modo que introduzimos o campo basico definido pela equaçăo $(4,15$ ], isto

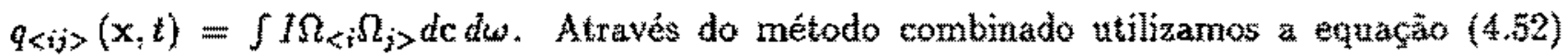
para obter um sistema de equaçoes envolvendo os deviantes do tensor pressão $p_{\langle i j\rangle}, q\langle i j\rangle e$ do gradiente de velocidade $\frac{\partial v_{\langle}}{\partial z_{y>}}$ [veja equaçóes (4.201) e (4.202)]. Na resoluçäo deste sistema necessitamos representar, bem como iaverter, o tensor de quarta ordem dependente do campo magnétice $A\langle i j\rangle\langle k\rangle$. Isto é feito, com detalhes, no apendice $B$. Com estas operaçoes realizadas

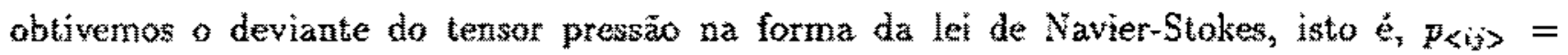

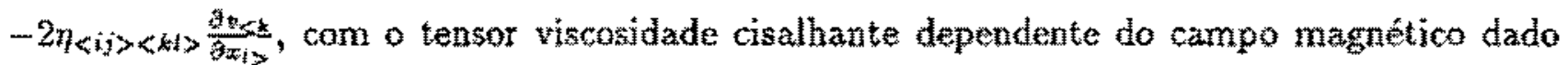
pela equaçäo $(4,226)$.

Sem perda de generalidade, consideramos o campo magnético orientado na direçăo $x$ de um sistema de coordenadas cartesianas, de modo que as cinco componentes independentes do tensor viscosidade cisalhante säo obtidas. As relaçôe entre as componentes do tensor pressāo

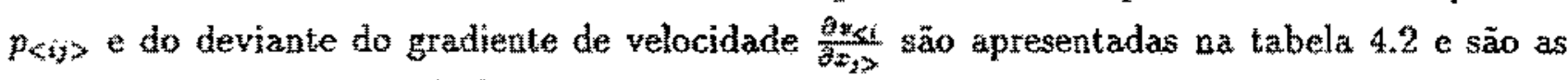
mesmas encontradas em [60], obthidas através de considerafónes de simetria e das relaços de Onsager.

Com a polarizaçâo $\Omega_{<i} \Omega_{j>}$ o coeficiente de viscosidade longitudinal $\eta_{1}$ näo á afetado pelo campo magnético, de modo que a variação relativa $\frac{\Delta \eta_{0}}{\eta_{0}}=0$ [49]; assim, outras polarizaçōes devern ser incluidas na teoria a fim de estabelecer o comportamento do coeficiente $\eta_{1}$ em termos do campo magnético aplicado. Os dados experimentais mostram que as variaçôes relativas de 箠 săo muito pequenas [50].

No gráfico da figura 4.8 são apresentadas as variaçöes relativas para os quatro coeficientes de viscosidade cisalhante; os coeficientes $\eta_{1}$ e $\eta_{2}$ sa que săo medidos. As curvas apresentarn o mesmo comportamento daquelas obtidas em [50].

\section{Coeficiente de Viscosidade Volumétrica}

O coeficiente de viscosidade volumétrica não é afetado pelo campo magnético se con" siderarmos na teoria somente a polarizaçüo $\Omega_{\alpha_{i}} \Omega_{j\rangle}$. Esta dependencia pode ser alcançada de incluirnos as polarizaçöes $C_{<i} C_{j>} \Omega_{k}$ on $C_{k i} C_{j}, \Omega_{<k} \Omega_{3>}[49]$.

๘ 


\section{Capítulo 5}

\section{Equações Linearizadas de Burnett}

\subsection{Introduçäo}

A primeira equaçăo constitutiva para o tensor pressăo com termos além daqueles que constituem a lei de Navier-Stokes é devida a Maxwell [22], gue procurou explicar a força de radiômetro usando a teoria cinética de gases (veja Introduçấ). Maxwell concluiu que o deviante do tensor pressäo $p_{<j y}, \dot{e}$ proporcional ao deviante do gradiente de segunda ordem da temperatura $\frac{\partial^{2} T}{\partial x_{i} T ; x ;}$.

Para gases monoatômicos rarefeitos, a equação constitutiva completa para o tensor pressäo $p_{i j}$ envolvendo derivadas de segunda ordem dos campos básicos (densidade, velocidade e temperatura) bern como produtos e quadrados derivadas de primeira ordem, foi obtida por Burnett [10]. A equaçăo constitutiva para o fluxo de calor foi obtida por Chapman e Cowling [9]. Na literatura elas são sắn conhecidas como equaçoes de Bumeth.

As equaçôes de Burnett são importantes no estudo de värios fenómenos tratando de gases rarefeitos como o efeito Scott [24] (veja Introduçâ) e a propagaçăo de ondas [66].

Nosso objetivo neste capitulo é determinar as equaçes linearizadas de Burnett para gases poliatômicos. Fazemos isto de duas maneiras distintats: através da teoria cinética de gases e segundo uma teoria fenomenológica.

Na teoria cinética de gases determinamos a terceira aproximaçăo linearizada para a funçă de distribuiçăo utilizando o método de Chapman-Enskog para resolver a equaçăo de Boltzmann. As moléculas do gás são consideradas como esferas rigidas, perfeitamente elásticas e perfeitamente rugosas que é o modelo de Bryan desenvolvido no capítulo 1.

As equaçón linearizadas de Burnett são tambêm obtidas através de uma teoria fenomenolö. gica a partir dos resultados da teoria termodinâmica estendida para gases poliatömicos, desen" volvida por Kremer [26, 27]. Nesta teoria o estado macroscópico do gás é caracterizado pelos dezessete campos excalares: densidade de massa, velocidade, tensor pressão, fuxo de calor translacional, densidade de energia da variavel interna fposteriormente jdentificada com a ener gía rotacional da molécula) e o fuxo de energia da variànel interna (posteriormente jdentificado 
com o fuxo de calor rotacional do gás).

Para resolvermos o sistema de equaçöes envolvendo os campos básicos empregamos o método jterativo de Maxwell [28] da teoria cinética. Com a primeíra iteração decorrem as les de Navier. Stokes e Fourier; com a segunda iteração obtemos as equaçôs linearizadas de Burnett.

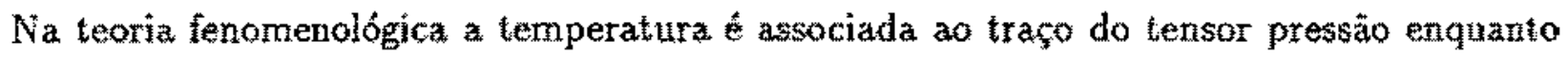
que na teoria cinética ela é associada à energia específica total da molécula Ao relacionarmos estas temperaturas podemos comparar as duas teorias. Além disso, colocamos a densidade de entropia e o fluxo de entropia em termos da temperatura e da pressào dinâmica definidas na teoria cinética e discutimos o papel desempenhado pelas temperaturas nas teorias cinética e fenomenológica, respectivamente.

A equaçào constitutiva para o tensor pressão de um găs poliatômico apresenta três termos que inexistem para um g guts monoatômico rarefeito. Um deles é ben conhecido pois está associ. ado ao divergente da velocidade do gás, tendo como coeficiente a viscosidade volumétrica. Os outros dois säo denominados de pressão térmica pressão de densidade e säo, respectivamente. proporcionais aos laplacianos da temperatura e da densidade. Estes termos também aparezem nas equaçốes constitutivas para um gás monoatómico moderadamente denso [67].

No desenvolvimento da teoria cinética empregamos os resultados do método de ChapmanEnskog desenvolvidos no capítulo 3 , enquanto que para a teoria fenomenológica nos baseamos nos resultados de [26, 27]. Os principais resultados deste capitulo esta apresentados em [68].

\subsection{Teoria Cinética}

\subsubsection{Segunda Aproximação para a Função de Distribuição}

Nesta seçäo nos reportamos aos principais resultados obtidos com o método de Chapman. Enskog desenvolvido no capítulo 3 , onde consideramos a segunda aproximaça $f^{(1)}=f^{(0)} \phi^{(1)}$ para a funçăo de distributçăo $f(x, c, \omega, t)$.

Para um gás poliatônico corn moléculas modeladas como esferas rugosas, a funçăo de dìs trĭbuiçăo é soluçâo da equação de Boltzmann (1.59) que, na ausência de forças e torques texter. nos. exrrita como

$$
\frac{\partial f}{\partial t}+c_{i} \frac{\partial f}{\partial x_{i}}=\int\left(f_{1}^{*} f^{*}-f_{1} f\right) a^{2}(\mathrm{~g} \cdot \mathrm{k}) d \mathrm{k} d \mathrm{c}_{1} d \omega_{1}
$$

Corno nos capitulos anteriores, empregamos a notaçăo:

$$
\begin{array}{ll}
f_{1}^{*}=f_{1}\left(x, c_{1}^{*}, \omega_{1}^{*}, t\right), & f_{1}=f\left(x, c_{1}, \omega_{1}, t\right) \\
f^{*}=f\left(x, c^{*}, \omega^{*}, t\right), & f=f\left(x, c_{,}, t\right),
\end{array}
$$

onde as grandezas com asterisco denotam o fato de que näo há colisäo inversa entre as moléculas. 
No método de Chapman-Enskog a solução para a equação (5.1) é suposta ser da forma

$$
f=f^{(0)}\left(1+\phi^{(1)}+\phi^{(2)}+\cdots\right)=f^{(0)}+f^{(1)}+f^{(2)}+\cdots
$$

onde a primeira aproximação $f^{(0)}$ é a função de distribuição para o equilíbrio

$$
f^{(0)}=\frac{\varrho}{m} \frac{(m I)^{3 / 2}}{(2 \pi k T)^{3}} \exp \left\{-\left(\frac{m C^{2}}{2 k T}+\frac{I \Omega^{2}}{2 k T}\right)\right\}
$$

e $f^{(1)}=f^{(0)} \phi^{(1)}, f^{(2)}=f^{(0)} \phi^{(2)}, \cdots$, são aproximações sucessivas para a função de distribuição de equilibrio $f^{(0)}$. As funçōes $\phi^{(i)}$ são denominadas de desvios da função $f^{(0)}$.

Niesta teoria a energia específica $\varepsilon$ é dada por

$$
\varepsilon=\varepsilon^{T}+\varepsilon^{R}
$$

onde $\varepsilon^{T}$ representa a energia translacional específica e $\varepsilon^{R}$ a energia interna rotacional específica. Como estamos tratando de uma teoria clássica na qual o princípio da equipartição da energia é válido temos que $\varepsilon^{T}=\varepsilon^{R}=\frac{3}{2} \frac{k}{m} T$ e, portanto

$$
\varepsilon(T)=3 \frac{k}{m} T .
$$

Verificamos na seção 3.2 que a segunda aproximação para a função de distribuição é dada pela a equação (3.64),

$$
\begin{gathered}
f^{(1)}=f^{(0)} \phi^{(1)}=f^{(0)}\left\{-\frac{2}{5}\left(\frac{m}{k T}\right)^{2}\left[\frac{\lambda^{T}}{\varrho}\left(\frac{m C^{2}}{2 k T}-\frac{5}{2}\right)+\frac{5}{3} \frac{\lambda^{R}}{\varrho}\left(\frac{I \Omega^{2}}{2 k T}-\frac{3}{2}\right)\right] C_{i} \frac{\partial T}{\partial x_{i}}\right. \\
\left.-\frac{\eta}{\varrho}\left(\frac{m}{k T}\right)^{2} C_{i} C_{j} \frac{\partial v_{<i}}{\partial x_{j>}}-\frac{\eta_{v}}{\varrho} \frac{m}{k T}\left(\frac{m C^{2}}{2 k T}-\frac{I \Omega^{2}}{2 k T}\right) \frac{\partial v_{r}}{\partial x_{r}}\right\}
\end{gathered}
$$

que é uma solução linearizada envolvendo somente gradientes de primeira ordem da temperatura e da velocidade.

Na equação (5.7) os coeficientes $\lambda^{T}, \lambda^{R}, \lambda, \eta$ e $\eta_{v}$ são identificados com os coeficientes de transporte para o gás e dados, respectivamente, pelas equações (3.57), (3.59), (3.60), (3.62) e (3.63), as quais reproduzimos a seguir:

- coeficiente de condutividade térmica translacional

$$
\lambda^{T}=\frac{225}{16 a^{2}}\left(\frac{k^{3} T}{\pi m}\right)^{1 / 2} \frac{(\kappa+1)^{3}(2 \kappa+1)}{\left(102 \kappa^{3}+101 \kappa^{2}+75 \kappa+12\right)}
$$

- coeficiente de condutividade térmica rotacional

$$
\lambda^{R}=\frac{9}{4 a^{2}}\left(\frac{k^{3} T}{\pi m}\right)^{1 / 2} \frac{(\kappa+1)^{2}(19 \kappa+3)}{\left(102 \kappa^{3}+101 \kappa^{2}+75 \kappa+12\right)}
$$


- coeficiente de condutividade térmica

$$
\lambda=\lambda^{T}+\lambda^{n}=\frac{9}{16 a^{2}}\left(\frac{k^{3} T}{\pi m}\right)^{1 / 2} \frac{(\kappa+1)^{2}\left(50 \kappa^{2}+151 \kappa+37\right)}{\left(102 \alpha^{3}+101 \kappa^{2}+75 x+12\right)}
$$

- coeficiente de viscosidade cisalhante

$$
\eta=\frac{15}{8 a^{2}}\left(\frac{m k T}{\pi}\right)^{1 / 2} \frac{(\kappa+1)^{2}}{(13 \kappa+6)}
$$

- coeficiente de viscosidade volumétrica

$$
\eta_{k}=\frac{1}{32 a^{2}}\left(\frac{m k T}{\pi}\right)^{1 / 2} \frac{(\kappa+1)^{2}}{\kappa}
$$

Com a segunda aproxjmaçäo (5. 7 ) para a funçăo de distribuição obtemos a equaçăo constitutiva para o terssor pressäo

$$
p_{i j}=\int m C_{i} C_{j} f d \mathrm{c} d \omega=\left(e \frac{k}{m} T-\eta \frac{\partial \tau_{r}}{\partial x_{r}}\right) \delta_{i j}-2 \eta \frac{\partial n_{\zeta i}}{\partial x_{j\rangle}}
$$

e a equaça constitutiva para o vetor fuxo de calor

$$
q_{i}=\int\left(\frac{1}{2} m C^{2}+\frac{1}{2} I \Omega^{2}\right) C_{i} f d c d \omega=-\left(\lambda^{T}+\lambda^{R}\right) \frac{\partial T}{\partial x_{i}}=-\lambda \frac{\partial T}{\partial x_{i}}
$$

que representam, respectivamente, as leis de Navier-Stokes e de Fourier para o gás poliatômico.

\subsubsection{Terceira Aproximação para a Função de Distribuiçâo}

Para obter as equaçoes de Burnet para um gấ poliatómico vamos escrever a funçăo de distributçào (5.3) na forma

$$
f=f^{(0)}\left(1+\phi^{(1)}+\phi^{(2)}\right)=f^{(0)}+f^{(1)}+f^{(2)}
$$

onde $f^{11)}$ é a segunda aproximação para a função $f^{(0)}$ dada pela equação (5.7) e $f^{(2)}$ é a terceira aproximação a ser determinada.

Consideremos inieialmente a descriçăo macroscópica de um gás poliatômico através da teoria termodinämica de cinco campos escalares: densidade de massa $\varrho(x, t)$, densidade de momento linear $\varrho v_{i}(\mathrm{x}, t)$ e temperatura $T(\mathrm{x}, t)$. Na teoria cinética estes campos säo definidos por

$$
\begin{aligned}
& \varrho(\mathbf{x}, t)=\int m f(\mathbf{x}, \mathbf{c}, \omega, t) d \mathrm{c} d \omega \\
& p^{v}(x, t)=\int m C_{i} \int(x, c, \omega \cdot l) d \mathrm{c} d \omega
\end{aligned}
$$




$$
T(\mathbf{x}, t)=\frac{m}{3 k \rho} \int\left(\frac{1}{2} m C^{2}+\frac{1}{2} I \Omega^{2}\right) f d c d \omega,
$$

com equaçöes de balanço dadas por $(3.7)$ a $(3.9)$.

$$
\begin{aligned}
& \frac{\partial \underline{p}}{\partial t}+\frac{\partial q_{i}}{\partial z_{i}}=0
\end{aligned}
$$

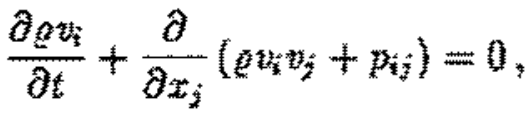

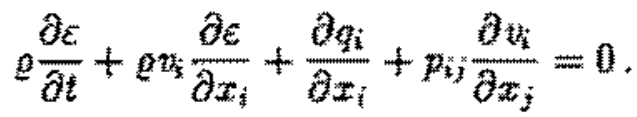

Estas equaçôes nāo constituem um sistema fechado de equaçöes para os campos básicos pois contétrn os termos constitutivos tensor pressän, definido por

$$
p_{i j}=\int m C_{i} C_{j} f d \mathrm{c} d \omega
$$

e o vetor fluxo de calor, definido por

$$
q_{i}=\int\left(\frac{1}{2} m C^{2}+\frac{1}{2} I \Omega^{2}\right) C_{i} f d \mathbf{c} d \omega
$$

os quais devem ser determinados em termos dos cempos básicos $\varrho(x, t), v_{i}(x, t)$ e $T(x, t)$.

Por sua vez; a determinaçä́o dos termos constitutivos $p_{i j}$ e $q_{i}$ depende do conhecimento da segunda aproximação $f^{(2)}$ para a funçăo de distribuição $f(x, c, \omega, t)$. Para determiná-la, vamos substituir a equaçäo (5.15) na equaçäo de Boltzmann $(5.1)$; então, obtemos

$$
\begin{aligned}
& \frac{\partial}{\partial t}\left(f^{(0)}+f^{(1)}\right)+c \frac{\partial}{\partial x_{i}}\left(f^{(0)}+f^{(1)}\right)=\int\left[f^{*(0)}\left(f_{1}^{*(1)}+f_{1}^{*(2)}\right)\right. \\
& \left.+f_{1}^{*(0)}\left(f^{*(1)}+f^{(2)}\right)-f^{(0)}\left(f_{1}^{(1)}+f_{1}^{(2)}\right)-f_{1}^{(0)}\left(f_{1}^{(1)}+f_{1}^{(2)}\right)\right] a^{2}(\mathrm{~g} \cdot \mathrm{k}) d \mathrm{k} d \mathrm{c}_{1} d \mathrm{w}_{1},
\end{aligned}
$$

onde mantivemos: no primetro membro desta equação somente as derivadas das funçotes fol e $f^{(1)}$ pois săo elas que geram os desvios $\phi^{(1)}=f^{(1)} / f^{(0)}$ e $\phi^{(2)}=f^{(2)} / f^{(0)}$ da tunçấ de distribuica $f^{(0)}$; no segundo membro, somente os termos lineares em $f^{(1)}$ a $f^{(2)}$ pois estamos

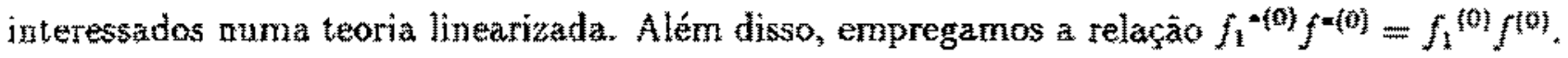

A equação (5.24) pode ser escrita na forma compacta

$$
\mathcal{D}\left(f^{(0)}+f^{(1)}\right)=I^{*}\left[\phi^{(1)}\right]+I^{*}\left[\omega^{(2)}\right]
$$

onde iniroduzimos os operadores

$$
\mathcal{D}=\frac{\partial}{\partial t}+c_{i} \frac{\partial}{\partial x_{\imath}}
$$




$$
T^{*}[\Phi]=\int f_{1}^{(0)} f^{(0)}\left(\Phi_{1}^{*}+\Phi^{*}-\Phi_{1}-\Phi\right) a^{2}(\mathrm{~g} \cdot \mathrm{k}) d \mathrm{k} t \mathrm{c}_{1} d \omega_{\mathrm{y}}
$$

Se multiplicarmos a equação $(5.25)$ por uma função arbitrária $\varphi(x, c, \omega, t)$ e integrarmos nas velocidades $\mathrm{c}$ e $\omega$, obtemos

$$
\begin{gathered}
\int \varphi D\left(f^{(0)}+f^{(1)}\right) d \mathbf{c} d \omega=\int \varphi T^{*}\left[\phi^{(1)}\right] d c d \omega+\int \varphi T^{*}\left[\phi^{(2)}\right] d c d \omega= \\
=\int \phi^{(1)} T[\varphi] d c d \omega+\int \phi^{(2)} \mathcal{I}[\varphi] d c d \omega
\end{gathered}
$$

onde

$$
I[\varphi]=\int f_{1}^{(0)} f^{(0)}\left(\varphi_{1}^{\prime}+\varphi^{*}-\varphi_{1}-\varphi\right) a^{2}(g \cdot \mathbf{k}) d \mathbf{k} d \mathrm{c}_{1} d \omega_{1}
$$

A segunda igualdade da equação (5.28) e obtida atrave da equação (3.24).

Se identificarmos a funçấo arbitrátia $p(x, c, t 2, t)$ com os invariantes de soma $\psi=m, m c_{i}$ e $\frac{1}{2} m c^{2}+\frac{1}{2} I \omega^{2}$, a equaçào $(5.28)$ se reduz a

$$
\int w \mathcal{D}\left(f^{(0)}+f^{(1)}\right) d c d w=0
$$

Ao aplicarmos o operador definido pela equação (5.26) às equaços $(5.4)$ e $(5.7)$, obtemos

$$
\begin{aligned}
& \int \psi f^{(0)}\left\{\frac{1}{e} \frac{\partial e}{\partial t}+\frac{1}{T}\left(\frac{m C^{2}}{2 k T}+\frac{m \Omega^{2}}{2 k T}-3\right) \frac{\partial T}{\partial t}+\frac{m}{k T} C_{i} \frac{\partial v_{i}}{\partial t}\right. \\
& +\left(C_{i}+v_{i}\right)\left[\frac{1}{\varrho} \frac{\partial \varrho}{\partial x_{i}}+\frac{1}{T}\left(\frac{m C^{2}}{2 k T}+\frac{I \Omega^{2}}{2 k T}-3\right) \frac{\partial T}{\partial x_{i}}+\frac{m}{k T} C_{i} \frac{\partial v_{j}}{\partial x_{i}}\right] \\
& +\frac{2}{g}\left(\frac{m}{k T}\right)^{2}\left[\frac{\lambda^{T}}{5}\left(\frac{5}{2}-\frac{m C^{2}}{2 k T}\right)+\frac{\lambda^{2}}{3}\left(\frac{3}{2}-\frac{I \Omega^{2}}{2 k T}\right)\right] C_{i} \frac{\partial^{2} T}{\partial t \partial x_{i}} \\
& -\frac{m}{e k T}\left[\frac{m}{k T} \eta C_{i} C_{j} \frac{\partial^{2} v_{\langle i}}{\partial t \partial x_{j\rangle}}+\eta_{*}\left(\frac{m C^{2}}{2 k T}-\frac{M M^{2}}{2 k T}\right) \frac{\partial^{2} v_{i}}{\partial t \partial x_{i}}\right] \\
& +\left(C_{i}+w_{i}\right)\left[\frac{2}{2}\left(\frac{m}{k T}\right)^{2}\left[\frac{\lambda^{T}}{3}\left(\frac{5}{2}-\frac{m C^{2}}{2 k T}\right)+\frac{\lambda^{R}}{3}\left(\frac{3}{2}-\frac{I \Omega^{2}}{2 k T}\right)\right] C_{j} \frac{\partial^{2} T}{\partial x_{i} \partial x_{i}}\right. \\
& \left.\left.-\frac{1}{e}\left(\frac{m}{k T}\right)^{2}\left[\eta C_{k} C_{j} \frac{\partial^{2} v_{<j}}{\partial x_{s} \partial x_{k>}}+\frac{k T}{m} \eta_{v}\left(\frac{m C^{2}}{2 k T}-\frac{I \Omega^{2}}{2 k T}\right) \frac{\partial^{2} \eta_{j}}{\partial x_{i} \partial x_{j}}\right]\right\}\right\} d \mathrm{c} d \omega=0 .
\end{aligned}
$$

Ao igualarmos na equaçào $(5.31)$ a função ${ }^{\text {ta }}$ aos invariames de soma $m, m c_{i}$ e $\frac{1}{2} m c^{2}+\frac{1}{2} / w^{2}$ e realizarmos as integraçỏes nas velocidades, obtemos as seguintes equaçós de balanço:

- balanço de massa

$$
\frac{\partial \varrho}{\partial t}+\frac{\partial \rho u_{i}}{\partial x_{i}}=0
$$


- balanço de densidade de momento linear

$$
e \frac{\partial v_{i}}{\partial t}+g y_{j} \frac{\partial v_{i}}{\partial x_{j}}+\frac{\partial}{\partial x_{i}}\left(\frac{k}{m_{m}} T\right)-2 \eta \frac{\partial^{2} v_{\langle i}}{\partial x_{j} \partial x_{j\rangle}}-\eta_{v} \frac{\partial^{2} v_{j}}{\partial x_{j} \partial x_{j}}=0
$$

- balarnço de densidade de energia

$$
3 e \frac{k}{m} \frac{\partial T}{\partial t}+3 \varrho \frac{k}{m} \frac{\partial T}{\partial x_{i}}+e \frac{k}{m} T \frac{\partial v_{i}}{\partial x_{i}}-\lambda \frac{\partial^{2} T}{\partial x_{i} \partial x_{i}}=0
$$

As equaçöes (5.32) a (5.34) represtatum as equaçoes linearizadas para um flúdo de Navier-

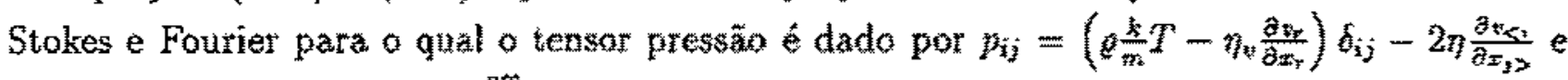

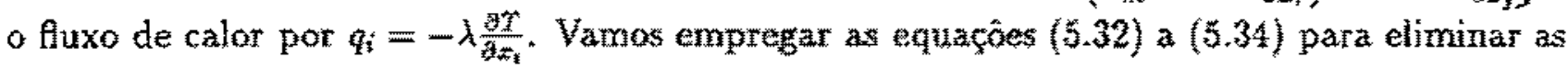
derivadas temporais da equaçă (5.31); podemos entäo escrever a equaçào (5.25) como:

$$
\begin{aligned}
& f^{(0)}\left\{-\frac{1}{3}\left(\frac{m C^{2}}{2 k T}+\frac{m n^{2}}{2 k T}\right) \frac{\partial n_{i}}{\partial x_{i}}+\frac{m}{k T} C_{i} C_{3} \frac{\partial v_{i}}{\partial x_{i}}+\frac{1}{T}\left(\frac{m C^{2}}{2 k T}+\frac{m O^{2}}{2 k T}-4\right) C_{i} \frac{\partial T}{\partial x_{i}}\right. \\
& +\left(\frac{m}{3 k \eta}\left(\lambda^{T}+\lambda^{R}\right)\left(\frac{m C^{2}}{2 k T}+\frac{7 \Omega^{2}}{2 k T}-3\right)+\frac{\eta_{w}}{k T}\left(\frac{m C^{2}}{2 k T}-\frac{1 \Omega^{2}}{2 k T}\right)\right. \\
& \left.+\frac{2}{3 \varrho}\left(\frac{m}{k T}\right)^{2} C^{2}\left[\frac{\lambda^{T}}{5}\left(\frac{5}{2}-\frac{m C^{2}}{2 k T}\right)+\frac{\lambda^{n}}{3}\left(\frac{3}{2}-\frac{T \Omega^{2}}{2 k T}\right)\right]\right\} \frac{\partial^{2} T}{\partial x_{i} \partial x_{i}} \\
& +\frac{2}{e}\left(\frac{m}{k T}\right)^{2}\left[\frac{\lambda^{T}}{5}\left(\frac{3}{2}-\frac{m C^{2}}{2 k T}\right)+\frac{\lambda^{R}}{3}\left(\frac{3}{2}-\frac{M \Omega^{2}}{2 k T}\right)+\frac{k}{2 m} \eta\right] C_{i} C_{j} \frac{\partial^{2} T}{\partial x_{\langle i} x_{j\rangle}} \\
& -\frac{4}{5} \frac{m}{g k T} \eta\left(\frac{m C^{2}}{2 k T}-\frac{5}{2}\right) C_{i} \frac{\partial^{2} v_{\langle i}}{\partial x_{j} \partial x_{j\rangle}}-\left(\frac{m}{k T}\right)^{2} \frac{\eta}{\ell} C_{k i} C_{j} C_{k>} \frac{\partial^{2} v_{k j}}{\partial x_{i} \partial x_{k>}} \\
& +\frac{2}{3} \frac{T}{\varrho}\left(\frac{m}{k T}\right)^{2}\left[\frac{\lambda^{T}}{5}\left(\frac{m C^{2}}{2 k T}-\frac{5}{2}\right)+\frac{\lambda^{R}}{3}\left(\frac{1 \Omega^{2}}{2 k T}-\frac{3}{2}\right)-\frac{3 k}{2} \frac{k}{m} \eta_{v}\left(\frac{m C^{2}}{2 k T}-\frac{I \Omega^{2}}{2 k T}-1\right)\right] C_{i} \frac{\partial^{2} v_{j}}{\partial x_{i} \partial m_{j}} \\
& \left.+\frac{m}{k Q^{2} T} \eta_{u} C_{i} C_{j} \frac{\partial^{2} \varrho}{\partial x_{\langle i} \partial x_{j\rangle}}+\frac{\eta_{v}}{\varrho^{2}}\left(\frac{m C^{2}}{2 k T}-\frac{I \Omega^{2}}{2 h T}\right) \frac{\partial^{2} \varrho}{\partial x_{i} \partial x_{i}}\right\}=T^{*}\left[\phi^{(1)}\right]+T^{*}\left[\phi^{(2)}\right] *
\end{aligned}
$$

que é a equaçäo integral linearizada para os desvios $\phi^{(1)}$ e $\phi^{(2)}$.

$O$ desvio $\phi^{(1)}$ é solução da equação integral (3.32) a qual pode ser escrita tomo

$$
f^{(0)}\left\{-\frac{1}{3}\left(\frac{m C^{2}}{2 k T}+\frac{I \Omega^{2}}{2 k T}\right) \frac{\partial v_{i}}{\partial x_{i}}+\frac{m}{k T} C_{i} C_{j} \frac{\partial \nu_{i}}{\partial x_{j}}+\frac{C_{i}}{T}\left(\frac{m C^{2}}{2 k T}+\frac{I \Omega^{2}}{2 k T}-4\right) \frac{\partial T}{\partial x_{i}}\right\}=T^{*}\left[\frac{b}{\partial n}\right] .
$$

Assim, se subtrairmos a equaçăo (5.36) da (5.35), resulta

$$
f^{(0)}\left\{\int \frac{m}{3 k n}\left(\lambda^{T}+\lambda^{R}\right)\left(\frac{m C^{2}}{2 k T}+\frac{I n^{2}}{2 k T}-3\right)+\frac{n w}{g T}\left(\frac{m C^{2}}{2 k T}-\frac{I n^{2}}{2 k T}\right)\right.
$$




$$
\begin{gathered}
\left.+\frac{2}{3 \varrho}\left(\frac{m}{k T}\right)^{2} C^{2}\left[\frac{\lambda^{T}}{5}\left(\frac{5}{2}-\frac{m C^{2}}{2 k T}\right)+\frac{\lambda^{R}}{3}\left(\frac{3}{2}-\frac{I \Omega^{2}}{2 k T}\right)\right]\right\} \frac{\partial^{2} T}{\partial x_{i} \partial x_{i}} \\
+\frac{2}{\varrho}\left(\frac{m}{k T}\right)^{2}\left[\frac{\lambda^{T}}{5}\left(\frac{5}{2}-\frac{m C^{2}}{2 k T}\right)+\frac{\lambda^{R}}{3}\left(\frac{3}{2}-\frac{I \Omega^{2}}{2 k T}\right)+\frac{k}{2 m} \eta\right] C_{i} C_{j} \frac{\partial^{2} T}{\partial x_{<i} x_{j>}} \\
-\frac{4}{5} \frac{m}{\varrho k T} \eta\left(\frac{m C^{2}}{2 k T}-\frac{5}{2}\right) C_{i} \frac{\partial^{2} v_{<i}}{\partial x_{j} \partial x_{j>}}-\left(\frac{m}{k T}\right)^{2} \frac{\eta}{\varrho} C_{<i} C_{j} C_{k>} \frac{\partial^{2} v_{<j}}{\partial x_{i} \partial x_{k>}} \\
+\frac{2}{3} \frac{T}{\varrho}\left(\frac{m}{k T}\right)^{2}\left[\frac{\lambda^{T}}{5}\left(\frac{m C^{2}}{2 k T}-\frac{5}{2}\right)+\frac{\lambda^{R}}{3}\left(\frac{I \Omega^{2}}{2 k T}-\frac{3}{2}\right)-\frac{3}{2} \frac{k}{m} \eta_{v}\left(\frac{m C^{2}}{2 k T}-\frac{I \Omega^{2}}{2 k T}-1\right)\right] C_{i} \frac{\partial^{2} v_{j}}{\partial x_{i} \partial x_{j}} \\
\left.+\frac{m}{k \varrho^{2} T} \eta_{v} C_{i} C_{j} \frac{\partial^{2} \varrho}{\partial x_{<i} \partial x_{j>}}+\frac{\eta_{v}}{\varrho^{2}}\left(\frac{m C^{2}}{2 k T}-\frac{I \Omega^{2}}{2 k T}\right) \frac{\partial^{2} \varrho}{\partial x_{i} \partial x_{i}}\right\}=\mathcal{I}^{*}\left[\phi^{(2)}\right],
\end{gathered}
$$

que é a equação integral para o desvio $\phi^{(2)}$.

A equação (5.37) pode ser escrita, em termos de polinômios de Sonine (veja Apêndice D) na seguinte forma:

$$
\begin{gathered}
f^{(0)}\left\{-\frac{m}{3 \varrho k T}\left\{\frac{8}{5} \lambda^{T} S_{1 / 2}^{(2)}\left(\beta C^{2}\right)+\left(\lambda^{R}-\lambda^{T}+3 \frac{k}{m} \eta_{v}\right)\left[S_{1 / 2}^{(1)}\left(\beta C^{2}\right)-S_{1 / 2}^{(1)}\left(\beta \Omega^{2}\right)\right]\right.\right. \\
\left.+\frac{4}{3} \lambda^{R} S_{1 / 2}^{(1)}\left(\beta C^{2}\right) S_{1 / 2}^{(1)}\left(\beta \Omega^{2}\right)\right\} \frac{\partial^{2} T}{\partial x_{i} \partial x_{i}}-\frac{\eta_{v}}{\varrho^{2}}\left[S_{1 / 2}^{(1)}\left(\beta C^{2}\right)-S_{1 / 2}^{(1)}\left(\beta \Omega^{2}\right)\right] \frac{\partial^{2} \varrho}{\partial x_{i} \partial x_{i}} \\
+\frac{2}{\varrho}\left(\frac{m}{k T}\right)^{2}\left[\frac{\lambda^{T}}{5} S_{5 / 2}^{(1)}\left(\beta C^{2}\right)+\frac{\lambda^{R}}{3} S_{5 / 2}^{(1)}\left(\beta \Omega^{2}\right)-\frac{1}{5}\left(\lambda^{T}+\frac{5}{2} \frac{k}{m} \eta\right) S_{5 / 2}^{(0)}\left(\beta C^{2}\right)\right. \\
\left.-\frac{2}{3} \lambda^{R} S_{5 / 2}^{(0)}\left(\beta \Omega^{2}\right)\right] C_{<i} C_{j>} \frac{\partial^{2} T}{\partial x_{<i} \partial x_{j>}}+\frac{m}{\varrho^{2} k T} \eta S_{5 / 2}^{(0)}\left(\beta C^{2}\right) C_{i} C_{j} \frac{\partial^{2} \varrho}{\partial x_{<i} x_{j>}} \\
+\frac{4}{5} \frac{m}{\varrho k T} \eta S_{3 / 2}^{(1)}\left(\beta C^{2}\right) C_{i} \frac{\partial^{2} v_{<i}}{\partial x_{j} x_{j>}}-\frac{2}{3} \frac{T}{\varrho}\left(\frac{m}{k T}\right)^{2}\left[\frac{1}{5}\left(\lambda^{T}-\frac{15}{2} \frac{k}{m} \eta_{v}\right) S_{3 / 2}^{(1)}\left(\beta C^{2}\right)\right. \\
\left.+\frac{1}{3}\left(\lambda^{R}+\frac{9}{2} \frac{k}{m} \eta_{v}\right) S_{3 / 2}^{(1)}\left(\beta \Omega^{2}\right)-\frac{1}{3}\left(\lambda^{R}-\frac{9}{2} \frac{k}{m} \eta_{v}\right) S_{3 / 2}^{(0)}\left(\beta \Omega^{2}\right)\right] C_{i} \frac{\partial^{2} v_{j}}{\partial x_{i} \partial x_{j}} \\
\left.-\frac{\eta}{\varrho}\left(\frac{m}{k T}\right)^{2} S_{7 / 2}^{(0)}\left(\beta C^{2}\right) C_{<i} C_{j} C_{k>} \frac{\partial^{2} v_{j}}{\partial x_{i} \partial x_{k}}\right\} \\
=\int f_{1}^{(0)} f^{(0)}\left(\phi_{1}^{(2)^{\prime}}+\phi^{(2)^{\prime}}-\phi_{1}^{(2)}-\phi^{(2)}\right) a^{2}(\mathrm{~g} \cdot \mathbf{k}) d \mathbf{k} d \mathrm{c}_{1} d \omega_{1}
\end{gathered}
$$

onde $\beta=m / 2 k T$.

A solução geral para o desvio $\phi^{(2)}$ da equação integral (5.38) consiste de uma combinação linear da solução da equaçào homogènea com uma solução particular da mesma equação. A soluçảo da equação homogênea é formada por uma combinação linear dos invariantes de soma. A soluçào particular pode ser construída através de uma combinação linear de segundos gradientes dos campos básicos tendo como coeficientes polinômjos de Sonine nas velocidades $\mathrm{C}$ e $\boldsymbol{\Omega}$. Assim. podemos escrever que 


$$
\begin{aligned}
& \phi^{(2)}=\sum_{r, s=0}^{\infty}\left[d_{r s}^{(1)} S_{1 / 2}^{(r)}\left(\beta C^{2}\right) S_{1 / 2}^{(d)}\left(\beta \Omega^{2}\right) \frac{\partial^{2} T}{\partial x_{i} \partial x_{i}}+d_{r s}^{(2)} S_{i / 2}^{(r)}\left(\beta C^{2}\right) S_{1 / 2}^{(s)}\left(\beta \Omega^{2}\right) \frac{\partial^{2} \underline{\partial}}{\partial x_{i} \partial x_{i}}\right.
\end{aligned}
$$

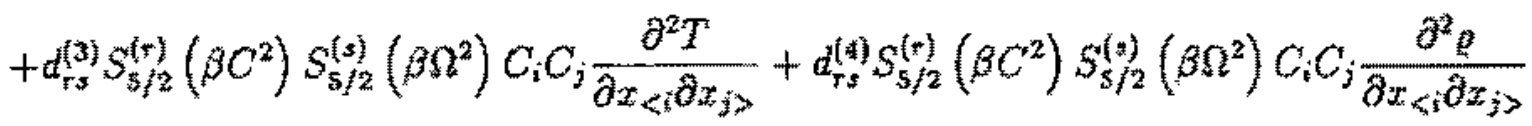

$$
\begin{aligned}
& +d_{r s}^{(5)} S_{3 / 2}^{(r)}\left(\beta C^{2}\right) S_{1 / 2}^{(s)}\left(\beta \Omega^{2}\right) C_{i} \frac{\partial^{2} v_{<j}}{\partial x_{j} \partial x_{j\rangle}}+d_{r s}^{(6)} S_{3 / 2}^{(+)}\left(\beta C^{2}\right) S_{3 / 2}^{(s)}\left(\beta \Omega^{2}\right) C_{i} \frac{\partial^{2} v_{j}}{\partial x_{i} \partial x_{j}} \\
& \left.+d_{* A}^{(n)} S_{7 / 2}^{(r)}\left(\beta C^{2}\right) S_{7 / 2}^{(s)}\left(\beta \Omega^{2}\right) C_{\langle i} C_{j} C_{k>} \frac{\partial^{2} v_{\langle j}}{\partial x_{j} \partial x_{k>}}\right]+\alpha^{(1)}+\alpha_{i}^{(2)} C_{i}+\alpha^{(3)}\left(C^{2}+\frac{1}{m} n^{2}\right)
\end{aligned}
$$

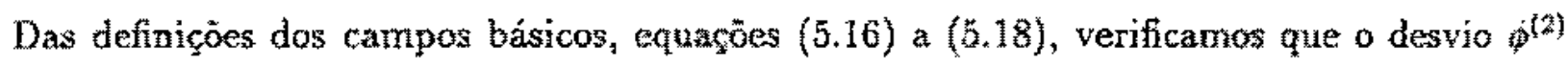
esta restrito à condiçầ de vínculo

$$
\int \psi^{3} s^{(0)} \phi^{(2)} d c d w=0
$$

onde $w$ representa os invariantes de soma.

Se substituimos a equação (5.39) na (5.40) e identificarmos a funçäo soma, após realizamos as integrạ̧öes nas velocidades $\mathrm{c}$ e w com o emprego da condição de ortogonalidade para os polinômios de Sonine, obtemos seguintes relaçòes entre coeficlentes da equaçäa (5.39):

- $\psi=m$

$$
\alpha^{(1)}+6 \frac{k T}{m} \alpha^{(3)}+d_{00}^{(1)} \frac{\partial^{2} T}{\partial x_{i} \partial x_{i}}+d_{00}^{(2)} \frac{\partial^{2} Q}{\partial x_{i} \partial x_{i}}=0
$$

- $\psi=m c_{i}$

$$
\alpha_{3}^{(2)}+d_{00}^{(5)} \frac{\partial^{2} y_{<i}}{\partial x_{j} \partial x_{j\rangle}}+d_{00}^{(5)} \frac{\partial^{2} z_{i}}{\partial x_{i} x_{j}}=0
$$

$* \psi^{*}=\frac{1}{2} m C^{2}+\frac{1}{2} l w^{2}$

$$
2 \alpha^{(1)}+16 \frac{k T}{m} \alpha^{(3)}+\left(2 d_{00}^{(1)}-d_{10}^{(1)}-d_{0 t}^{(1)}\right) \frac{\partial^{2} T}{\partial x_{i} \partial x_{i}}+\left(2 d_{00}^{(3)}-d_{10}^{(2)}-d_{01}^{(2)}\right) \frac{\partial^{2} \varrho}{\partial x_{i} \partial x_{i}}=0
$$

Vamos considerar a primeira aproximação para a soluçầ (5.39) que contem somente termos em $C^{2}$ e $\Omega^{2}$ na expansấo infinita de polinômios de Sonine; assim, se determinarmos os coeficientes $\alpha^{(1)}, \alpha_{i}^{(3)}$ e $\alpha^{(3)}$ do sistema de equaçoes $(5.41)$ a (3.43). substitutimos na equaça (5.40) e mantivermos apenas os termos que fornecem contribuiçôs näo nulas nas integraçōes para 0 lensor pressâo pi, para o vetor fluxo de calor $q_{\mathrm{i}}$ obternos: 


$$
\begin{aligned}
& \phi^{|2|}=d_{1}\left(\frac{m C^{3}}{2 k T}-\frac{I n^{2}}{2 k T}\right) \frac{\partial^{2} T}{\partial x_{i} \partial x_{i}}+d_{2}\left(\frac{m C^{2}}{2 k T}-\frac{I^{2}}{2 k T}\right) \frac{\partial^{2} g}{\partial x_{i} \partial x_{i}}+d_{3} C_{i} C_{j} \frac{\partial^{2} T}{\partial x_{\langle i} \partial x_{j\rangle}} \\
& +k_{4} C_{i} C_{j} \frac{\partial^{2} Q}{\partial x_{\langle i} \partial x_{j\rangle}}+\left[d_{5}\left(\frac{3}{2}-\frac{1 \Omega^{2}}{2 k T}\right)+d_{6}\left(\frac{5}{2}-\frac{m C^{2}}{2 k T}\right)\right] C_{i} \frac{\partial^{2} v_{\langle i}}{\partial x_{j} \partial x_{j\rangle}} \\
& +\left[d_{7}\left(\frac{3}{2}-\frac{M \Omega^{2}}{2 k T}\right)+d_{8}\left(\frac{5}{2}-\frac{m C^{2}}{2 k T}\right)\right] C_{i} \frac{\partial^{2} v_{j}}{\partial x_{i} \partial x_{j}}
\end{aligned}
$$

onde redefinimos:

$$
\begin{aligned}
& d_{1}=2\left(d_{01}^{(1)}-d_{10}^{(1)}\right), \quad d_{2}=2\left(d_{01}^{(2)}-d_{10}^{(2)}\right), \quad d_{3}=d_{000}^{(3)}, \quad d_{4}=d_{00}^{(1)} \\
& d_{s}=d_{01}^{(5)}, \quad d_{6}=d_{10}^{(6)}, \quad d_{7}=d_{0 \mathrm{I}}^{(7)}, \quad d_{8}=d_{10}^{(8)}
\end{aligned}
$$

Ao substituirmos o desvio $\phi^{(2)}$ da equaçäo (5.44) na equaçäo integral (5.37) e igualarmos os coeficientes dos gradientes de mesma ordem dos campos, obtemos as seguintes equaçós integrais:

$$
\begin{aligned}
& \frac{1}{3} f^{(0)}\left\{\frac{m\left(\lambda^{T}+\lambda^{R}\right)}{\varrho k T}\left(\frac{m C^{2}}{2 k T}+\frac{I \Omega^{2}}{2 k T}-3\right)+3 \frac{\eta_{t}}{\varrho T}\left(\frac{m C^{2}}{2 k T}-\frac{I \Omega^{2}}{2 k T}\right)\right. \\
& \left.+\frac{2}{e}\left(\frac{m}{k T}\right)^{2}\left[\frac{\lambda^{2}}{5}\left(\frac{5}{2}-\frac{m C^{2}}{2 k T}\right)+\frac{\lambda^{R}}{\varrho}\left(\frac{3}{2}-\frac{I \Omega^{2}}{2 k T}\right)\right] C^{2}\right\}=d_{1} \tau\left[\frac{m \Omega^{2}}{2 k T}-\frac{m C^{2}}{2 k T}\right] \\
& \frac{\eta_{2}}{Q^{2}} f^{(0)}\left(\frac{m C^{2}}{2 k T}-\frac{I \Omega^{2}}{2 k T}\right)=d_{2} T\left[\frac{I \Omega^{2}}{2 k T}-\frac{m C^{2}}{2 k T}\right] \\
& \frac{2}{e}\left(\frac{m}{k T}\right)^{2} f^{(0)}\left[\frac{\lambda^{T}}{5}\left(\frac{5}{2}-\frac{m C^{2}}{2 k T}\right)+\frac{\lambda^{R}}{3}\left(\frac{3}{2}-\frac{m \Omega^{I}}{2 k T}\right)+\frac{k}{m} \frac{\eta}{\varrho}\right] C_{<i} C_{j\rangle}=d_{3} I\left[C_{<i} C_{j\rangle}\right] \\
& \frac{m}{2^{2} m T} j^{(0)} C_{k i} C_{j\rangle}=d_{4} T\left[C_{\langle i} C_{j\rangle}\right] \\
& -\frac{4}{5} \frac{m}{g} \frac{\eta}{\varrho}\left(\frac{m C^{2}}{2 k T}-\frac{5}{2}\right) C_{i}=d_{5} I\left[\left(\frac{5}{2}-\frac{I \Omega^{2}}{2 k T}\right) C_{i}\right]+d_{5} T\left[\left(\frac{5}{2}-\frac{m C^{2}}{2 k T}\right) C_{i}\right] \\
& -\frac{T}{\varrho}\left(\frac{m}{k T}\right)^{2} f^{10 !}\left[\frac{k}{m} \eta_{v}\left(\frac{m C^{2}}{2 k T}-\frac{m \Omega^{2}}{2 k T}-1\right)+\frac{2}{15} \lambda^{T}\left(\frac{5}{2}-\frac{m C^{2}}{2 k T}\right)+\frac{2}{9} \lambda^{R}\left(\frac{3}{2}-\frac{I \Omega^{2}}{2 k T}\right)\right] C_{i}
\end{aligned}
$$




$$
=d_{7} I\left[\left(\frac{5}{2}-\frac{m C^{2}}{2 k T}\right) C_{i}\right]+d_{8} \mathcal{I}\left[\left(\frac{5}{2}-\frac{m C^{2}}{2 k T}\right) C_{i}\right]
$$

Multiplicando as equações (5.46) e (5.47) por $\left(\frac{m C^{2}}{2 k T}-\frac{3}{2}\right) d c d \omega$, as equações (5.48) e (5.49) por $C_{<r} C_{s>} d c d \omega$ e integrando as equações resultantes nas velocidades, obtemos:

$$
\begin{gathered}
d_{1}=-\left(\frac{m}{k T}\right)^{2}\left(3 \frac{k}{m} \eta_{v}+\lambda^{R}-\lambda^{T}\right), \\
d_{2}=-3 \frac{m}{k T} \frac{\eta_{v}^{2}}{\varrho^{3}} \\
d_{3}=-\frac{m^{2}}{k^{2} T^{3}}\left(\eta-\frac{2}{5} \frac{m}{k} \lambda^{T}\right) \frac{\eta}{\varrho^{2}}, \\
d_{4}=-\frac{m}{k T} \frac{\eta^{2}}{\varrho^{3}} .
\end{gathered}
$$

Multiplicando a equação (5.50) sucessivamente por $\left(\frac{5}{2}-\frac{m C^{2}}{2 k T}\right) C_{i} d \mathrm{c} d \omega$ e $\left(\frac{5}{2}-\frac{I \Omega^{2}}{2 k T}\right) d \mathrm{c} d \omega \mathrm{e}$ realizandos as integrações nas velocidades, resultam:

$$
(5 \kappa) d_{5}-(17 \kappa+4) d_{6}=\frac{3}{2 \sqrt{\pi}} \frac{(\kappa+1)^{2}}{m a^{2}}\left(\frac{m}{\varrho}\right)^{2}\left(\frac{m}{k T}\right)^{3 / 2} \eta
$$

e

$$
d_{6}=\frac{3\left(2 \kappa^{2}+2 \kappa+1\right)}{5 \kappa} d_{5}
$$

Resolvendo este sistema de equações, temos:

$$
d_{5}=-\frac{15}{2 \sqrt{\pi}} \frac{1}{m a^{2}}\left(\frac{m}{\varrho}\right)^{2}\left(\frac{m}{k T}\right)^{3 / 2} \frac{\kappa(\kappa+1)^{2}}{\left(102 \kappa^{3}+101 \kappa^{2}+75 \kappa+12\right)} \eta,
$$

e

$$
d_{6}=-\frac{9}{2 \sqrt{\pi}} \frac{1}{m a^{2}}\left(\frac{m}{\varrho}\right)^{2}\left(\frac{m}{k T}\right)^{3 / 2} \frac{(\kappa+1)^{2}\left(2 \kappa^{2}+2 \kappa+1\right)}{\left(102 \kappa^{3}+101 \kappa^{2}+75 \kappa+12\right)} \eta .
$$

Ao multiplicarmos a (5.51) sucessivamente por $\left(\frac{5}{2}-\frac{m C^{2}}{2 k T}\right) C_{i} d c d \omega$ e $\left(\frac{5}{2}-\frac{1 \Omega^{2}}{2 k T}\right) d c d \omega$ e realizarmos as integraçōes nas velocidades, obtemos o seguinte sistema de equações:

$$
(5 \kappa) d_{i}-(17 \kappa+4) d_{8}=\frac{15}{8 \sqrt{\pi}} \frac{(\kappa+1)^{2}}{m a^{2}}\left(\frac{m}{\varrho}\right)^{2}\left(\frac{m}{k T}\right)^{3 / 2}\left(\eta_{v}-\frac{2}{15} \frac{m}{k} \lambda^{T}\right)
$$

$$
3\left(2 \kappa^{2}+2 \kappa+1\right) d_{7}-(5 \kappa) d_{8}=\frac{9}{8 \sqrt{\pi}} \frac{(\kappa+1)^{2}}{m a^{2}}\left(\frac{m}{\varrho}\right)^{2}\left(\frac{m}{k T}\right)^{3 / 2}\left(\eta_{v}+\frac{2}{9} \frac{m}{k} \lambda^{T}\right)
$$


com soluģōes

$$
\begin{gathered}
d_{3}=\frac{3}{8 \sqrt{\pi} \frac{m}{a^{2} \ell^{2}}\left(\frac{m}{k T}\right)^{3 / 2}} \frac{(\kappa+1)^{2}}{\left(102 \kappa^{3}+101 \kappa^{2}+75 \kappa+12\right)}\left[25 \kappa\left(\frac{2}{15} \frac{m}{k} \lambda^{T}-\eta\right)\right. \\
\left.+3(17 \kappa+4)\left(\frac{2 m}{9} \lambda^{R}+\eta\right)\right],
\end{gathered}
$$

e

$$
\begin{gathered}
d_{B}=\frac{3}{8 \sqrt{\pi}} \frac{m}{a^{2} t^{2}}\left(\frac{m}{k T}\right)^{3 / 2} \frac{(\kappa+1)^{2}}{\left(102 \kappa^{3}+101 k^{2}+75 k+12\right)}\left[\left(2 \kappa^{2}+2 k+1\right)\left(\frac{2}{15} \frac{m}{k} \lambda^{m}-\eta\right)\right. \\
\left.+\kappa\left(\frac{2 m}{9} \frac{m}{k} \lambda^{k}+\eta\right)\right] .
\end{gathered}
$$

Assim, a segunda aproximaçã⿰o para a funçă de distribuiçấo pode ser escrita, através da equaçăo $(5.44)$, como

$$
\begin{gathered}
f^{(2)}=f^{(0)} \phi^{(2)}=f^{(0)}\left\{d_{1}\left(\frac{m C^{2}}{2 k T}-\frac{I \Omega^{2}}{2 k T}\right) \frac{\partial^{2} T}{\partial x_{i} \partial x_{i}}+d_{2}\left(\frac{m C^{2}}{2 k T}-\frac{I \Omega^{2}}{2 k T}\right) \frac{\partial^{2} Q}{\partial x_{i} \partial x_{i}}\right. \\
+d_{3} C_{i} C_{3} \frac{\partial^{2} T}{\partial x_{i} \partial x_{i}}+d_{4} C_{i} C_{3} \frac{\partial^{2} \ell}{\partial x_{<i} \partial x_{i}}+\left[d_{5}\left(\frac{3}{2}-\frac{I \Omega^{2}}{2 k T}\right)+d_{3}\left(\frac{5}{2}-\frac{m C^{2}}{2 k T}\right)\right] C_{i} \frac{\partial^{2} v_{<i}}{\left.\partial x_{j} \partial x_{j}\right\rangle} \\
\left.+\left[d_{7}\left(\frac{3}{2}-\frac{I \Omega^{2}}{2 k T}\right)+d_{8}\left(\frac{5}{2}-\frac{m C^{2}}{2 k T}\right)\right] C_{i} \frac{\partial^{2} v_{j}}{\partial x_{i} \partial x_{j}}\right\}
\end{gathered}
$$

com os coeficientes $d_{i}$ dados pelas equactes $(5.52),(5.53),(5.54),(5.55),(5.58),(5.59),(5.62)$ e $(5.63)$.

Tendo determinado a funça $f^{(2)}\left(x_{*}, w, t\right)$, podemos calculat o tensor pressão $p_{i j}$ e o vetor fluxo de calor $q_{i}$ bem como os respectivos coeficientes de transporte. E o que faremos na seça seguinte.

\subsubsection{Coeficientes de Transporte para as Equaçōes Lỉnearizadas de Burnett}

Com a terceira aproximaçào para a funçà̀ de distribuiç̆ăo (5.15) o tensor pressão, definido pela equuâão (5.22), é escrito como

$$
p_{i j}=\int m C_{3} C_{j} f^{(0)}\left(1+\phi^{(1)}+\phi^{(2)}\right) d \mathrm{c} d \omega
$$

Tendo em conta a soluçäo para a segunda aproximaçầo dada pela equaçào (5.13). podemos escrever a equaça anterior como 


$$
p_{i j}=\left(\frac{k}{m} T-\eta_{v} \frac{\partial w_{i}}{\partial x_{r}}\right) \hat{o}_{i j}-2 \eta \frac{\partial w_{k}}{\partial x_{j\rangle}}+\int m C_{i} C_{j} f^{(0)} \phi^{(j)} d c d \omega
$$

onde $\eta$ e thy sẫo dados, respectivamente, pelas equaçōes (5.11) e (5.12).

Inserindo o desvio $\phi^{(2)}$ da equaça (5.64) na equação anterior realizando as integraçoes nas velocidades, obtemos

$$
\begin{aligned}
p_{i j}= & \left(e \frac{k}{m} T-\eta_{v} \frac{\partial v_{\gamma}}{\partial x_{r}}-\beta_{1} \frac{\partial^{2} \ell}{\partial x_{r} \partial x_{r}}-\rho_{2} \frac{\partial^{2} T}{\partial x_{7} \partial x_{r}}\right) \delta_{i j} \\
& -2 \eta \frac{\partial v_{<i}}{\partial x_{j\rangle}}-2 \beta_{3} \frac{\partial^{2} \ell}{\partial x_{<i} \partial x_{j\rangle}}+\beta_{4} \frac{\partial^{2} T}{\partial x_{<i} \partial x_{j\rangle}}
\end{aligned}
$$

onde

$$
\begin{aligned}
& \beta_{1}=3\left(\frac{\eta}{\varrho}\right)^{2}, \quad \beta_{2}=\frac{m}{k T}\left(\lambda^{R}-\lambda^{T}+3 \frac{k}{m} \eta_{t}\right) \frac{\eta_{w}}{\varrho} \\
& \beta_{3}=\left(\frac{\eta}{\varrho}\right)^{2}, \quad \beta_{4}=\frac{4 m}{k T}\left(\lambda^{T}-\frac{5 k}{2 m} \eta\right) \frac{\eta}{\varrho} .
\end{aligned}
$$

Para o traço do tensor pressão, temos

$$
\frac{1}{3} p_{r s}=e \frac{k}{m} T+\Pi
$$

le modo que a equação (5.67) pode ser escrita como

$$
p_{i j}=\left(\frac{k}{m} T+\Pi\right) g_{i j}-2 \eta \frac{\partial v_{<i}}{\partial x_{j\rangle}}-2 \beta_{3} \frac{\partial^{2} \varrho}{\partial x_{\langle i} \partial x_{j\rangle}}+\beta_{i} \frac{\partial^{2} T}{\partial x_{\langle i} \partial x_{j\rangle}}
$$

onde a pressão dinâmica $\Pi$ é dada por

$$
\mathrm{\Pi}=-\frac{\partial v_{\psi}}{\partial x_{*}}-\beta_{1} \frac{\partial^{2} \underline{\partial}}{\partial x_{\tau} \partial x_{*}}-\beta_{2} \frac{\partial^{2} T}{\partial x_{\tau} \partial x_{\psi}}
$$

Ao compararnos a equação (5.67) com a expressäo para o tensor pressăo que decorre da segunda aproximaçäo para a funçäo de distribuiçäo dada pela equaçăo (5.13), verificamos que a primeira apresenta termos adicionais: no traço, um termo proporcional ao laplaciano da densidade denominado pressão de densidade um terno proporcional ao laplaciano da temperatura, denominato pressấo tétmica, enquanto que aa parte sem traço há dois termos proporcionais a gradientes tie segunda ordem da densidade e da temperatura, respectivarnente. Alén disso, verificamos das equações $(5.68)$ que os coeficienles $\beta_{1}$ e $\beta_{3}$ sho positivos semudefinidos enquanto que os coeficientes $\beta_{2}$ e $\beta_{4}$ permanecem com seus sinais indeterminados.

Com a terceira aproximasào para a funçào de distribuiçăo (5.15) o vetor fluxo de calor (5.23) pude ser escrito como 


$$
q=\int\left(\frac{m C^{2}}{2}+\frac{I \Omega^{2}}{2}\right) C_{i} f^{(0)}\left(1+\phi^{(1)}+\phi^{(2)}\right) d c d \omega
$$

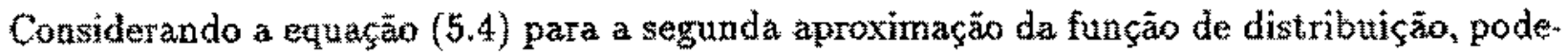
mos escrever a equação anterior como

$$
q_{i}=-\left(\lambda^{T}+\lambda^{R}\right) \frac{\partial T}{\partial x_{i}}+\int\left(\frac{m C^{2}}{2}+\frac{I \Omega^{2}}{2}\right) C_{i} f^{(0)} \phi^{(2)} d \mathrm{c} d w
$$

onde $\lambda^{T}$ e $\lambda^{*}$ são os coeficientes de condutividade térmica rotacional e translacional dados, respectivamente, pelas equacồes $(5.8)$ e $(5.9)$.

Se inserimos o desvio $\phi^{(2)}$ da equação (5.44) na equaçăo anterior e realizarmos as integraçōes nas velocidades, obtemos

$$
q_{i}=-\left(\lambda^{T}+\lambda^{A}\right) \frac{\partial T}{\partial x_{i}}+\gamma_{1} \frac{\partial^{2} v_{c i}}{\partial x_{j} \partial x_{j}}-\gamma_{i} \frac{\partial^{2} v_{j}}{\partial x_{i} \partial x_{i}}
$$

onde

$$
\gamma_{1}=\frac{4 m}{5} \frac{m}{\ell} \eta \lambda^{T}
$$

e

$$
\gamma_{2}=\frac{2}{15}\left(\frac{m}{k}\right)^{2} \frac{\lambda^{T}}{0}\left[\lambda^{T}+\frac{4}{15} \frac{(19 x+3)}{(2 x+1)(\kappa+1)} \lambda^{k}-\frac{3}{10} \frac{k}{m} \frac{\left(50 \kappa^{2}-\alpha+13\right)}{(2 \kappa+1)(\kappa+1)} \eta_{y}\right] .
$$

Comparando as equaçoes (5.14) e (5.74) para o vetor furo de calor verificamos que esta ütima apresenta termos adicionais proporcionais a gradientes de segund ordem da velocidade. Além disso, das equaçöes $(5.75)$ e $(5.76)$ verificamos que o coeficiente de transporte $\gamma$ é positivo semidefinido, enquanto que o sinal do coeficiente $\gamma_{2}$ permanece indeterninado.

Assim, através da teoria cinética de gases, determinamos as equaçōes linearizadas de Burnett (5.67) e (5.74) para um gás poliatômico com as moléculas consideradas como esferas rugosas. Observamos que os coeficientes destas equaçố \$ăo funçố da temperatura $T$ e da densidade e sendo que a massa $m_{1}$ o diâmetro a e o momento de inércia $I$ da molécula säo consideradas grandezas conhecidas.

No entanto, para aplicar os resultados obtjdos duas condiçoes devem ser preenchidas pelo gas poliatômico:

(i) as moléculas constituintes devern ser esfericamente simétricas, pois uthizamos o modelo de esferas rugosas:

(ii) a razäo $\gamma=c_{p} / c_{\mathrm{v}}$ entre os calores especificos do gás deve ser próxima de $4 / 3$, pois para moléculas corn seis graus de liberdade os calores especificos a volume e pressäo constantes sắn dados. respectivamente, por $\varepsilon_{\mathrm{r}}=3 \mathrm{k} / \mathrm{m}$ e $c_{\mathrm{p}}=4 \mathrm{k} / \mathrm{m}$. 
Estas condiçōes são verificadas, aproximadamente, pelos seguintes gases poliatômicos: metano $\left(\mathrm{CH}_{4}\right)$, tetrafluoreto de carbono $\left(\mathrm{CF}_{4}\right)$ e o metano- $\mathrm{d}_{4}\left(\mathrm{CD}_{4}\right)$ nos intervalos de temperatura apresentados na tabela 1.1. Na tabela 1.2 são apresentados valores característicos para os três gases mencionados.

Com os valores da tabela 1.2 verificamos, para os coeficientes de transporte dados pelas equaçōes (5.68), (5.75) e (5.76), que:

- para o $\mathrm{CH}_{4}$

$$
\begin{aligned}
& \beta_{1}>0, \quad \beta_{2}>0, \quad \beta_{3}>0 \\
& \gamma_{1}>0, \quad \gamma_{2}>0 .
\end{aligned}
$$

- para o $\mathrm{CF}_{4}$ e $\mathrm{CD}_{4}$

$$
\begin{aligned}
& \beta_{1}>0, \quad \beta_{2}>0, \quad \beta_{3}>0, \\
& \gamma_{1}>0, \quad \gamma_{2}<0 .
\end{aligned}
$$

Para um gás em repouso $\left(v_{i}=0\right)$ temos, da equação (5.71):

$$
\Pi=-\beta_{1} \frac{\partial^{2} \varrho}{\partial x_{r} \partial x_{r}}-\beta_{2} \frac{\partial^{2} T}{\partial x_{r} \partial x_{r}} .
$$

Considerando que os coeficientes $\beta_{1}$ e $\beta_{2}$ são positivos para o $\mathrm{CH}_{4}, \mathrm{CF}_{4}$ e $\mathrm{CD}_{4}$, podemos concluir que:

(j) a pressão dinâmica II diminui se a densidade $\varrho$ é uniforme e o laplaciano da temperatura $T$ é positivo, ou

(ii) a pressão dinâmica $\Pi$ diminui se a temperatura $T$ é uniforme mas o laplaciano da densidade $\varrho$ é positivo.

Estes efeitos ocorrem, também, para gases moderadamente densos [67], mas as pressōes aumentam sob as condições mencionadas.

A densidade de entropia $\varrho s$ e o fluxo de entropia $\phi_{i}$ para o gás poliatômico são dados. respectivamente, pelas equaçōes (3.128) e (3.130).

\subsection{Teoria Fenomenológica}

\subsubsection{Resultados de uma Teoria Termodinâmica Estendida}

Ima teoria fenomenológica para gases poljatômicos com uma variável interna e com base na termodinâmica estendida. foi formulada por Kremer $[26,27]$. A energia da variáve] interna 
pode ser identificada com a energia rotacional ou com a energía vibracional da molécula. Nesta seçäa, apresentaremos os principais resultados obtidos.

A teoria temodinamica estendidada de gases ideais com uma varyanel interna tem por objetivo a determinação dos dezessete campos escalares seguintes:

$$
\begin{aligned}
& \varrho \quad-\text { densidade de massa } \\
& v_{i}-\text { velocidade hidrodinâmica } \\
& p_{i j}-\text { tensor pressäo } \\
& q_{i}^{T}-\text { fuxo de calor translacional } \\
& g g-\text { densidade de energia da variável interna } \\
& g_{i}-\text { fluxo de energia da variável interna. }
\end{aligned}
$$

As equagcoes de balanço para estes campos säo:

$$
\begin{aligned}
& \frac{\partial \varrho}{\partial i}+\frac{\partial \varrho v_{i}}{\partial z}=0 \\
& e\left(\frac{\partial v_{i}}{\partial t}+v_{j} \frac{\partial v_{i}}{\partial x_{j}}\right)+\frac{\partial p_{j}}{\partial x_{i}}=\varrho F_{i}
\end{aligned}
$$

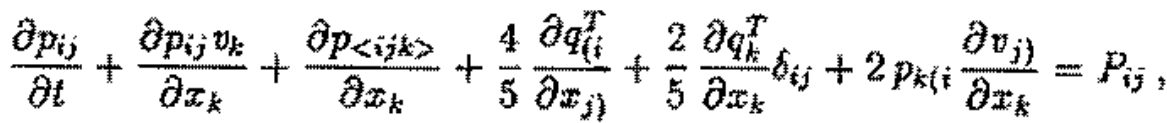

$$
\begin{aligned}
& \frac{\partial q_{i}^{T}}{\partial t}+\frac{\partial q_{i}^{T} v_{k}}{\partial x_{k}}+\frac{1}{2} \frac{\partial p_{i j j_{k}}}{\partial x_{k}}+p_{\langle i j k\rangle} \frac{\partial v_{i}}{\partial x_{k}}+\frac{2}{5} q_{i}^{T} \frac{\partial v_{j}}{\partial x_{i}}+\frac{7}{5} q_{j}^{T} \frac{\partial v_{i}}{\partial x_{j}} \\
& +\frac{2}{5} q_{i}^{T} \frac{\partial v_{k}}{\partial x_{k}}-\frac{p_{i j}}{\rho} \frac{\partial p_{j k}}{\partial x_{k}}-\frac{1}{2} \frac{p_{i j}}{\varrho} \frac{\partial p_{i k}}{\partial x_{k}}=P_{i}^{T} \\
& p\left(\frac{\partial g}{\partial t}+v_{i} \frac{\partial g}{\partial x_{i}}\right)+\frac{\partial g_{i}}{\partial x_{i}}=-\frac{1}{2} P_{i i} \\
& \frac{\partial g_{i}}{\partial t}+\frac{\partial g_{i} v_{k}}{\partial x_{k}}+\frac{\partial g_{i j}}{\partial x_{j}}+g_{j} \frac{\partial v_{i}}{\partial x_{j}}-g \frac{\partial p_{i j}}{\partial x_{j}}=P_{i}^{\prime}
\end{aligned}
$$

Estas equaçōes näo podem ser consideradas um sistema de equaçöes para os campos básicos (5.80) pois contém grandezas adicionais, as quais são:

$$
\begin{aligned}
& \text { psijk> } \quad \text { - deviante do fuxo do tensor pressão } \\
& \frac{1}{2} p_{i j z} \quad-\text { fluxo do nuxo de calor translacional } \\
& g_{i j} \text { - fluxo do fluxo de energia da variável interna } \\
& P_{i}, P_{\langle i j\rangle}, P_{i}^{T}, P_{i}^{T}-\text { termos de productato. }
\end{aligned}
$$


O tensor pressảo pode ser escrito na forma

$$
p_{i j}=p \delta_{i j}+p_{<i j>}
$$

onde $p_{\langle i\rangle}$ é seu deviante e $p=\frac{1}{3} p_{r}$ é a pressão hidrostática do gás.

A equaçẫo térmica de estado para um gás clássico é dada por

$$
p=\frac{\underline{g}}{m} k \hat{v}
$$

e energia translacional especifica é escrita como

$$
E^{T}=\frac{3}{2} \frac{k}{m} v_{3}
$$

onde denotamos por y temperatura do gás. Assim, estas duz grandezas estão relacionadas atraves de

$$
p=\frac{2}{3} e \varepsilon^{T}
$$

Desta forma, ao determinarmos o tensor pressão $p_{i j}$ da equaçäo (5.88) estamos também obtendo a densidade de energia especínca translacional $e^{T}$ cujo fluxo é dado pelo vetor fluxo de calor translacional $q_{i}^{T}$.

Podemos decompor a energia especfica g da variável interna em duas partes,

$$
y=z^{*}+\Delta
$$

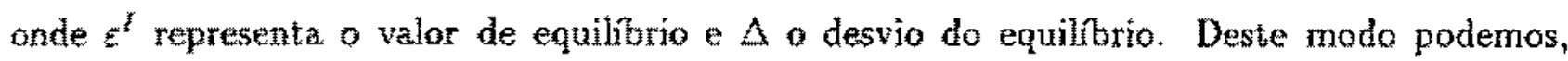
alterbativamente, considerar os dezessete campos básicos de (5.80) como sendo:

$$
\varrho, v_{i}, p_{i j}, q_{i}^{T}, \Delta \text { e } g_{i} .
$$

Com as equaçöes $(5.90)$ e (5.92) podemos escrever a energia especifica interna como

$$
\varepsilon=\varepsilon^{T}+g=\frac{3}{2} \frac{k}{m}+\varepsilon^{T}+\Delta
$$

Assumiremos que as grandezas constitutivas (5.87) dependem materialmente dos campos (5.93) enquanto que o valor de equilibrio da energia especifica da variavel interna $\xi^{\prime}$ depende somente da densidade de massa $\varrho$ e da pressäo $p$, ou seja

$$
\begin{gathered}
\left\{p_{<i j k>>} p_{i j j k}, g_{i j}, P_{i k}, P_{\langle i j\rangle}, p_{i}^{T}, P_{i}^{l}\right\}=C\left(g, v_{i}, p_{i j}, q_{i}^{T}, \Delta, g_{i}\right) \\
\varepsilon^{I}=\varepsilon^{l}(g, p) .
\end{gathered}
$$

A substituiçăo de (5.95) e (3.96) nas equaçöes de balango (5.81) a (5.86) conduz a um sistema de equaçoes de campo para os dezessete campos báxicos (5.93). Cada soluça deste sistema de equaçós é denominado de um processo termodinâmico. 
As funçöes constitutivas $C$ da equaçäo (5.95) são completamente gerais. Con o objetivo de restringi-las, Kremer [26, 27] empregou o princípio da entropia e o princípio da indiferença ao referencial [69].

O princípio da entropia estabelece que a desigualdade entrópica

$$
\frac{\partial \varrho s}{\partial t}+\frac{\partial}{\partial x_{i}}\left(\varrho s v_{i}+\phi_{i}\right) \geq 0
$$

É valida para todo processo termodinâmico. A densidade de entropia es e o fuxo de entropia $\phi$ são consideradas grandezas constitutivas que dependern dos campos básicos, ou seja

$$
\left\{\rho s, w_{i}\right\}=\mathcal{D}\left(\varrho, v_{i}, p_{i j}, q_{i}^{r}, \Delta, g_{i}\right)
$$

O principio da indiferença a referencial estabelece que as funçöes constitutivas $\mathcal{C}$ e das equaçoes (5.95) e (5.98) devem ter a mesma forma tanto em referenciais inerciais quanto em referenciais năo inerciais. Através deste princípio é possível mostrar que as funçöes constitutivas $\mathcal{C}$ e $D$ nào dependem dà velocidade $v_{i} \varepsilon_{y}$ além disso, säo funçôes isotrópicas das demaís variáveis especificadas pela equação (5.93).

Destu forma, as equaçöes $(5.95)$ e (5.98) podem ser escritas como

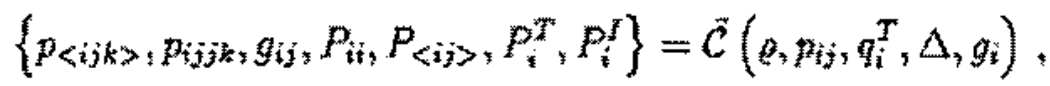

$$
\begin{aligned}
& \left\{\varrho s, \phi_{i}\right\}=\tilde{\mathcal{D}}\left(\varrho, p_{i j}, q_{i}^{T}, \Delta, g_{i}\right)
\end{aligned}
$$

sendo $\mathcal{C}$ e funçòes isotrópicas.

Nesta teoria, a temperatura é identificada com o fator integrante da forma pfaffana $\left(\left.d e\right|_{E}-\frac{p}{Q^{2}} d g\right)$, cuja integral é a entropia especifica em equilíbrio, ou seja

$$
\left.d s\right|_{E}=\frac{1}{v}\left(\left.d\right|_{E}-\frac{p}{e^{2}} d \theta\right)
$$

que é a equaçä̀ de Gibbs. Desta equaçăo decorre a condiçăo de integrabilidade

$$
\left(\frac{\partial \varepsilon}{\partial \varrho}\right)_{v}=\frac{1}{\partial^{2}}\left[p-\vartheta\left(\frac{\partial p}{\partial v}\right)_{q}\right] \text {. }
$$

Para um gás ideal, das equaçôes $(5.89)$ e $(5.90)$, conchümos que

$$
\left(\frac{\partial c^{I}}{\partial p}\right)_{\theta}=0
$$

Assim, a energia espectfica associada à varizvel interna independe da densidade $e_{4}$ isto é, depende somente da temperatura absoluta $\vartheta$. Deste modo, aquaçäo (5.94) para a energia especifica intema $z$ pode ser escrita como

$$
\varepsilon=\frac{3}{2} \frac{k}{m} \theta+\varepsilon^{I}(\vartheta)+\Delta
$$


O equilibrio é caracterizado como um processo termodinâmico no qual sâo nulas as as grandezas $p_{<i j\rangle}=p_{i j}-p \delta_{i j}, q_{i}, g_{i}$ e $\Delta$ bem como todos os termos de produçáo. Para protessos nas vizinhanças do equilbrio as equagoes constitutivas $(5.99)$, que decorrem do principio da entropia e do principio da indiferença ao referencial, são:

$$
\begin{aligned}
& p_{<i j k>}=0 \\
& p_{i ; k}=5 \frac{p^{2}}{2} \delta_{i k}+7 \frac{p}{p} p_{\langle i k\rangle} \\
& g_{i j}=\left(p \varepsilon^{\prime}+p \Delta\right) \delta_{i j}+\varepsilon^{l} p<i> \\
& P_{i}^{T}=\alpha_{1} g_{i}^{T}+\alpha_{2} g_{i}, \quad P_{\langle i j\rangle}=\sigma p_{\langle i\rangle\rangle} \\
& P_{i}^{i}=\chi_{1} q_{i}^{T}+\chi_{2} g_{i}, \quad P_{i}=\xi \Delta .
\end{aligned}
$$

onde $\alpha_{1}, \alpha_{2}, \chi_{1}, X_{2}, \sigma$ e $\xi$ säo coeficientes fenomenológicos os quais são hunçố de

A desigualdade entrópica (5.97) impöe as seguintes restriçoes aos coeficientes dos termos de produçäo, dados pelas equaç̧ōes (3.108) e (5.109):

$$
\begin{gathered}
\alpha_{1} \leq 0, \quad \chi_{2} \leq 0, \quad \sigma \leq 0, \quad \xi \geq 0, \\
\alpha_{1 X_{2}}-\frac{1}{10} \frac{m}{k} \varepsilon_{v}^{J}\left(\alpha_{2}+\frac{5}{2} \frac{k}{m} \frac{1}{\varepsilon_{0}^{I}} \chi_{1}\right)^{2} \geq 0
\end{gathered}
$$

onde $\varepsilon_{3}$ e o calor especifico da variável interna volume constante, dado por

$$
\varepsilon_{v}^{I}=\left(\frac{\partial \varepsilon^{t}}{\partial \theta}\right) .
$$

Por outro lado, as equaçóes constitutivas para a densidade de entropia $p s$ e para o fuxo de calor $\phi_{i}$, que são obtidas com o princípjo da entropia e do principio da indiferença ao referencial, sâo dadas por

e

$$
\begin{aligned}
& g s=g\left(\frac{k}{m} \ln \frac{v^{3 / 2}}{g}+\int \frac{\varepsilon_{y}^{l}(v)}{y} d y+a_{4}\right)+\frac{e}{v} \Delta-\frac{1}{2} \frac{e}{v^{2} E_{y}^{l}} \Delta^{2} \\
& -\frac{1}{d p^{q}} p_{\langle r s\rangle} p_{\langle\mathrm{r} s\rangle}-\frac{1}{5} \frac{e}{\left.p^{2}\right\rangle} a_{r}^{T} q_{r}^{T}-\frac{1}{2} \frac{1}{p^{2} \varepsilon_{q}^{T}} g_{i} g_{i}
\end{aligned}
$$

$$
\dot{\phi}_{i}=\frac{1}{\vartheta}\left(q_{i}^{T}+g_{i}\right)-\frac{1}{\vartheta^{2} \varepsilon_{\vartheta}^{l}} \Delta g_{i}-\frac{2}{5} \frac{1}{p \vartheta} p_{\langle i r\rangle} q_{F}^{T}
$$

onde $a_{4}$ é uma constante de integraçào.

$\mathrm{Na}$ seça seguinte identificaremos os coeficientes $\alpha_{3}, \alpha_{2}, x_{1}, x_{2}, \sigma$ e $\{$ das equaçoes (5.108) e (5.109). os quais säo denominados de coeficientes de uransporte. 


\begin{tabular}{|l|c|c|}
\hline & Teoria Cinética & Teoria fenomenológica \\
\hline \hline temperatura & $T$ & $\vartheta$ \\
\hline $\begin{array}{l}\text { traço do tensor } \\
\text { pressäo }\end{array}$ & $\frac{1}{3} p_{r r}=\varrho \frac{k}{m} T+I I$ & $\frac{1}{3} p_{r r}=\ell_{m}^{k \vartheta}$ \\
\hline $\begin{array}{l}\text { energia especifica } \\
\text { interna }\end{array}$ & $\varepsilon=\frac{3}{2} \frac{k}{m} T+\varepsilon^{T}(T)$ & $\varepsilon=\frac{3}{2} \frac{k}{m} \vartheta+\varepsilon(\vartheta)+\Delta$ \\
\hline
\end{tabular}

Tabela 5.1: Comparagäo entre as duas teorias.

\subsubsection{Comparação Entre Grandezas das Duas Teorias}

A tooria fenomenológica desenvolvida na seçâno anterior é anảloga a método dos momentos de Grad LII desenvolvido na teoria cinética. Se empregarmos as equaços de campo desta teoria juntamente com um esquema iterativo da teoria cinetica, denominado de iteraçầ Maxwelliana [28], podemos obter as equaçóes constitutivas para o deviante do tensor pressâo p pux> para o vetor fluxo de calor translacional $q_{i}^{T}$, para ofluxo de energia da variável interna $g_{t}$ e para o desvio do equilibrio $\Delta$ da energia especifica da varjavel interna $g$ [26].

Entretanto, do desenvolvimento apresentado na seção 5.2 e na subseça que näo é possivel comparar a teoria cinetica com a teoria tenomenológica pois há algumas diferenças entre ambas. As principais são apresentadas na tabela 5.1 .

Assim, verificamos que na teoria cinética o tensor pressão apresenta explícitamente o termo de näo-equilibrio (a pressão dinâmica II) enquanto que na teoria fenomenológica o termo de năo-equilibrio (o desvio $\Delta$ ) ocorre na expressão para a energia especifica internat.

A fim de que a equação (5.104) para a energia especfica interna total da teoria fenomenológica se reduza

$$
\varepsilon=\frac{3}{2 m} T+\varepsilon^{l}(T)
$$

vamos telacionar as temperaturas das duas teorias através de

$$
\hat{y}=T+\Upsilon \Delta \text {. }
$$

onde $\Upsilon$ é un coeficiente a ser determinado. Substituindo a equaça anterior na (5.104) e expandindo $E^{\prime}(T+\Upsilon \Delta)$ em série de Taylor até primeíra ordem, pois estamos interessados numa teoria linearizada, obtemos

$$
\varepsilon=\frac{3}{2} \frac{k}{n} T+\varepsilon^{l}(T)+\left(1+\varepsilon_{T}+\frac{3 k}{3 m} \Upsilon\right) \Delta
$$

onde introduzimos o calor especfico a volume constante associado variável interna

$$
E_{T}^{l}=\left(\frac{\partial \varepsilon^{I}}{\partial T}\right)_{0}
$$


Ao compararmos a equaçăo (5.117) com a $(5.115)$, concluimos que

$$
\Upsilon=-\frac{1}{\varepsilon_{T}^{I}+\frac{3}{2} \frac{k}{\pi^{2}}}
$$

Assim, a relação (5.116) entre as temperaturas, torna-se

$$
\vartheta=T-\frac{\Delta}{E^{I}+\frac{3}{3}}
$$

as quais são idêntỉcas para estados de equilforio.

Com o objetivo de comparat as equaçós de Burnet das duas teorias, vamos proceder a uma mudança de variáveis: ao invés de $\varepsilon$ e $\Delta$ da teoria fenomenológica vamos considerar $T$ e II da teoria cinética como novas variáveis.

Da definiçäo para o traço do tensor pressão da teoria fenomenológica

$$
\frac{1}{3} p_{x r}=p \frac{k}{m}
$$

e com a equaçäo $(5.120)$, temos

$$
\frac{1}{3} p_{r}=g \frac{k}{m} T-\frac{g \frac{k}{m}}{c_{T}^{l}+\frac{3}{3} \frac{k}{m}} \Delta .
$$

Comparando com a equação correspondente da teoria cinética

$$
\frac{1}{3} p_{s r}=e \frac{k}{n} T+\Pi
$$

conclumos que dectio $\Delta$ e a pressäo dinamica $I I$ estäo relacionados por

$$
\Delta=-\frac{\varepsilon_{t}+\frac{3}{2} \frac{k_{n}}{m}}{e^{\frac{k}{m}}} n
$$

Assim. a equação $(5.120)$ pode ser escrita como

$$
\eta=T+\frac{m}{k \varrho} \mathrm{I}
$$

a energia especifica assocjada à variável interna, como

$$
\varepsilon^{\prime}(\theta)=z^{\prime}(\eta)+\frac{m}{k g} \varepsilon_{T}^{I} \Pi
$$

\subsubsection{Equaçōes de Balanço Linearizadas}

Em termos das variáveis $T$ e $\Pi$, as equaçoes constitutivas $(5.105)$ a $(5.109)$ da teorla ferfomenologica podem ser escritas como:

$$
p_{\{1 j k\rangle}=0 \text {, }
$$




$$
\begin{aligned}
& p_{\text {i } i k}=5 \frac{k}{m} T\left(g \frac{k}{m} T+2 \Pi\right) \delta_{\xi k}+7 \frac{k}{m} T p_{\langle i k\rangle} \\
& g_{i j}=\left[\varepsilon^{l}(T) \frac{k}{m} T-\left(\frac{3}{2} \frac{k}{m} T-\varepsilon^{k}(T)\right) \Pi\right] \delta_{i j}+\epsilon^{t}(T) p_{\langle i j\rangle} \\
& P_{i}^{T}=\alpha_{1} q_{i}^{r}+\alpha_{z} g_{i}, \quad P_{\langle i\rangle\rangle}=\sigma p_{\langle i j\rangle}, \\
& P_{i}^{l}=\chi_{1} q_{i}^{r}+\chi_{2} g_{i}, \quad P_{i i}=-\varepsilon \frac{1}{p} \frac{m}{k}\left(\varepsilon_{T}^{t}+\frac{3 k}{2 m}\right) \Pi,
\end{aligned}
$$

onde negligenciamos os termos nâo lineares nos campos.

Ern seguida vamos reescrever as equagóes de balanço (5.81) a (5.86) em termos das varia veuts $T$ e $I 1$ na forma linearizada, isto é, eliminamos produtos de vi com gradientes dos campos básicos ou produtos de gradientes destes carnpos. Assim, obtemos:

- Equaçảo de balanço linearizada para a energia específica total.

Tomando o traço da equação de baluaço (5.83) para o tensor press̃̃o, obtemos

$$
\frac{\partial p_{r r}}{\partial t}+p_{r r} \frac{\partial v_{i}}{\partial x_{i}}+2 \frac{\partial q_{i}}{\partial x_{i}}+\frac{2}{3} p_{r r} \frac{\partial v_{i}}{\partial x_{i}}=P_{r r}
$$

Das equações $(5.92),(5.104)$ e $(5.121)$ temos

$$
\frac{1}{3} p_{y}=\frac{2}{3} e(z-g)
$$

Substituindo a equaçăo (5.133) na (5.132) e adicionando à equaçăo resultante a equaçấo de balanço (5.85) para a energia específica da variźvel interna $g$, resulta

$$
e \frac{\partial \varepsilon_{i}}{\partial t}+\frac{\partial q_{i}^{T}}{\partial x_{i}}+\frac{\partial g_{i}}{\partial x_{i}}+e \frac{k}{m} T \frac{\partial v_{i}}{\partial x_{i}}=0
$$

Da equação $(\overline{3} .115)$, temos

$$
\frac{\partial \varepsilon}{\partial t}=\left(\frac{3}{2 m} T+\varepsilon_{T}\right) \frac{\partial T}{\partial t}
$$

e equaçầ (5.134) pode ser escritu como

$$
e\left(\frac{3}{2} \frac{k}{m} T+c_{T}^{i}\right) \frac{\partial T}{\partial t}+\frac{\partial q_{i}^{T}}{\partial x_{i}}+\frac{\partial g_{i}}{\partial x_{i}}+\rho \frac{k}{m} T \frac{\partial v_{i}}{\partial x_{i}}=0
$$

que representa equaça de balanço linearizada para a energia espectica total $\varepsilon=\frac{3}{2} \mathrm{k} T+$ (T). 
- Equaçấo de balanço linearizada para a pressão dinâmica.

Substituindo a equação $(5.131)_{2}$ na equaçăo (5.132) para o traço do tensor pressão, temos

$$
e \frac{k}{m} \frac{\partial T}{\partial t}+\frac{\partial \Pi}{\partial t}+\frac{2}{3} \frac{\partial q_{t}^{T}}{\partial s_{t}}=\frac{1}{3 k T}\left(\frac{3}{2} \frac{k}{2 m}+\varepsilon_{T}^{I}\right) \xi \Pi
$$

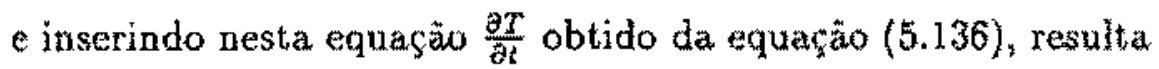

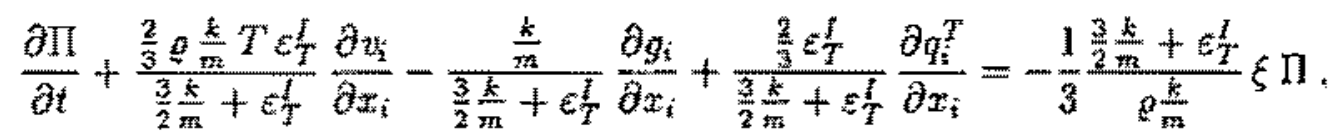

que é a equacảo de balanço linearizada para a pressão dinâmíca $\mathrm{II}$.

- Equaçăo de balanço linearizada para o deviante do tensor pressäo.

Tomando a parte sem traço da equaçăo de balanço (5.83) para o tensor pressäo e tendo

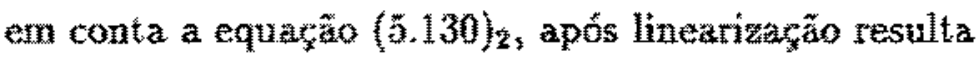

$$
\frac{\partial p_{\langle i j\rangle}}{\partial t}+\frac{4}{3 t} \frac{\partial q_{k i}^{T}}{\partial x_{j\rangle}}+2 g \frac{k}{m} T \frac{\partial n_{\langle i}}{\partial x_{j\rangle}}=g p_{\langle i j\rangle}
$$

que é a equação de balanço linẹarizada para o deviante do tensor pressão.

- Equaçào de balanģo linearizada para o fluxo de calor translacional.

Inserindo as equaçôes $(5.128)$ e (3.131) na equaçào para o fluxo de calor translacional $(5.84)$, após linearrização obtemos

$$
\frac{\partial q_{i}^{T}}{\partial t}+\frac{5}{2}\left(\frac{k}{m}\right)^{2} \varrho T \frac{\partial T}{\partial x_{i}}+\frac{5}{2} \frac{k}{m} T \frac{\partial \Pi}{\partial x_{i}}+\frac{k}{m} T \frac{\partial p_{\langle i \zeta\rangle}}{\partial x_{j}}=\alpha_{1} q_{i}^{T}+\alpha_{2} g_{i} .
$$

- Equaçăo de balanço linearizada para o fluxo de energia da varível interna.

Das equaçós $(5.86),(5.129),(5.131)$ e após linearizaçẵo concluimos que

$$
\frac{\partial g_{i}}{\partial t}+\varrho \frac{k}{m} T \varepsilon_{T}^{I} \frac{\partial T}{\partial x_{i}}-\frac{3}{2} \frac{k}{m} T \frac{\partial \Pi}{\partial x_{i}}=\chi_{1} q_{i}^{T}+\chi_{2} g_{i}
$$

Tendo obtido :s equaçóes de balanço para a pressăo dinámica II, para o deviante do tensor pressão p<y - para o fuxo de calor translacional $q_{i}{ }^{T}$ e para o fluxo de energia da variavel interna $g_{*}$ passaremos dateminaça dos coeftcientes de transporte atraves de um processo iterativo aplicado a este sistema de equaçòes. 


\subsubsection{Iteraçāo Maxwelliana e as Equaçōes Linearizadas de Burnett}

Com o objetivo de obter as equaçōes constitutivas para: a pressão dinâmica II, o deviante do tensor pressäo $p_{\langle i j\rangle}$ e para of fluxo de energia da variável interna $g_{i}$ empregaremos um método iterativo da teoria cinética denominado método iterativo de Maxwell [28].

A primeira iteração consiste em substituir no lado esquerdo das equaçoes de campo (5.138) a $(5.141)$ os valores de equilibrio

$$
\begin{aligned}
& I^{(0)}=0 \\
& p_{<i j\rangle}^{(0)}=0 \\
& q_{i}^{r(0)}=0 \\
& g_{i}^{(0)}=0
\end{aligned}
$$

e, em seguida, calcular os valores correspondentes à primeira iteração: $\mathrm{n}^{(1)}, p_{<i j>}^{(1)}, g_{i}{ }^{(1)}$ e $g_{i}{ }^{(1)}$.

Substituindo as equaçoes $(5.142),(5.144)$ e $(5.145)$ no lado esquerdo da equação de balanço (5.138), obtemos

$$
\frac{\frac{2}{3} e \frac{k}{m} T \varepsilon_{T}^{f}}{\frac{3}{2} \frac{k}{m}+\varepsilon_{T}^{l}} \frac{\partial v_{i}}{\partial x_{i}}=-\frac{1}{3} \frac{\frac{3}{2} \frac{k}{m}+\varepsilon_{T}^{l}}{e_{m}^{\frac{k}{m}}} \xi \Pi^{(n)}
$$

Assim. a pressão dinâmica pode ser escrita como

$$
\Pi^{(1)}=-\eta_{v} \frac{\partial v_{i}}{\partial x_{i}}
$$

onde identificamos o coeficiente de viscosidade volumétrica por

$$
\eta_{v}=2 \frac{e^{2}}{\xi}\left(\frac{k}{m}\right)^{2} \frac{T \varepsilon_{T}^{l}}{\left(\frac{3}{2}+\varepsilon_{T}^{I}\right)^{2}}
$$

Com a desigualdade $(5.110)_{4}$, concluimos que o coeficiente $\eta_{v}$ urna quantidade positiva semidefinida, isto é

$$
\eta_{u} \geq 0
$$

Das equaçôes $(5.143),(5.144)$ e $(5.139)$, temos que

$$
2 \frac{k}{m} T \frac{\partial u_{<\mathrm{i}}}{\partial x_{j\rangle}}=\sigma p\langle i\}
$$

ou

$$
p_{\langle i j\rangle}^{n !}=-2 \eta \frac{\partial v_{\langle i}}{\partial x_{j\rangle}}
$$

onde o coeficiente de viscosidade cisalhante é dado por

$$
\eta=-\frac{p}{\sigma}
$$


Con a desigualdade $(5.110)_{3}$ verificamos que o coeficiente de viscosidade cisalhante é uma grandeza positiva semidefnida, isto é

$$
7 \geq 0
$$

De (5.142) a (5.145), obtemos o seguinte sistema de equaçỏes:

$$
\begin{aligned}
& \frac{5}{2}\left(\frac{k}{m}\right)^{2} e^{T} \frac{\partial T}{\partial x_{i}}=\alpha_{1} q_{i}^{T(1)}+\alpha_{2} g_{i}^{(1)}
\end{aligned}
$$

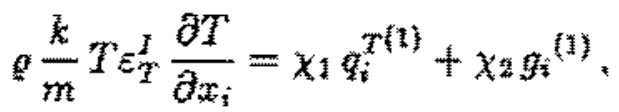

que tem como soluçöes

$$
q_{i}^{T^{(1)}}=-\lambda^{T} \frac{\partial T}{\partial x_{i}}
$$

e

$$
g_{i}^{(i)}=-\lambda \frac{\partial T}{\partial x_{i}}
$$

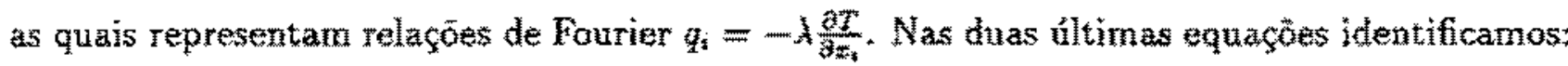
o coeficiente de condutividade térmica translacional, por

$$
\lambda^{T}=\frac{\frac{5}{2}\left(\frac{k}{m}\right)^{2} e T \chi_{2}-\frac{k}{m} \varepsilon_{T}^{J} \varrho T \alpha_{2}}{\alpha_{2} \chi_{1}-\alpha_{1} \chi_{2}},
$$

e o coeficiente de condutividade térmica associado à variável interna, por

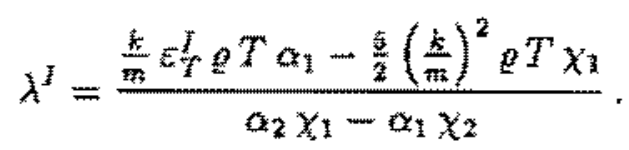

A segunda iteraçầ consiste em substituir no lado esquerdo das equaços de campo (5.138) a (5.141) as primeiras iteraçōes $\Pi^{(1)}, p_{<i j\rangle}^{(1)} q_{i}^{r(1)} g_{i}^{(1)}$ e proceder as respectivas linearizaçòes. Para eliminar as derivadas temporais destas equaçöes empregarenos. da equação (5.82)

$$
\frac{\partial v_{i}}{\partial t}=-\frac{1}{\rho} \frac{\partial p_{i j}}{\partial x_{j}}
$$

e da equarza $(5.136)$

$$
\frac{\partial T}{\partial t}=-\frac{1}{g\left(\frac{3}{2} \frac{k}{m} T+\varepsilon_{T}^{t}\right)}\left(\frac{\partial q_{i}^{T}}{\partial x_{i}}+\frac{\partial g_{i}}{\partial x_{i}}+\rho \frac{k}{m} \Upsilon \frac{\partial v_{i}}{\partial x_{i}}\right) .
$$

Substituindo a equação (5.147) no lado esquerdo da (5.138) e com o emprego da equaçà (5. 163 \} obtemos, após linearização, que a pressäo dinárnica é dada por 


$$
\Pi^{(2)}=-\eta_{v} \frac{\partial v_{i}}{\partial x_{i}}-\beta_{1} \frac{\partial^{2} e}{\partial x_{r} x_{r}}-\beta_{2} \frac{\partial^{2} T}{\partial x_{r} x_{r}},
$$

onde os coeficientes $\beta_{1}$ e $\beta_{2}$ săo dados por

$$
B_{1}=\frac{3}{2} \frac{\frac{k}{m}+\varepsilon_{T}^{I}}{\varepsilon_{T}^{I}} \frac{\eta^{2}}{\ell^{2}}
$$

e

$$
\beta_{2}=\frac{m}{e^{k T}}\left[\frac{3}{2} \frac{k}{m} \frac{\lambda^{I}}{\varepsilon_{T}^{I}}+\frac{\varepsilon_{T}^{I}+\frac{3}{2} \frac{k}{m}}{\varepsilon_{T}^{T}} \eta_{v}-\lambda^{T}\right] \eta_{v}
$$

Das equações (5.139), (5.151) e (5.163) resulta, após linearização, que o deviante do tensor pressão é dado por

$$
p_{<i j\rangle}^{(2)}=-2 \eta \frac{\partial v_{<i}}{\partial x_{j\rangle}}-2 \beta_{3} \frac{\partial^{2} \varrho}{\partial x_{<i} x_{j\rangle}}+\beta_{4} \frac{\partial^{2} T}{\partial x_{\langle i} x_{j\rangle}},
$$

onde

$$
\beta_{3}=\frac{\eta^{2}}{Q}
$$

e

$$
\beta_{4}=\frac{4}{3} \frac{m}{\rho k T}\left(\lambda^{T}-\frac{5}{2} \frac{k}{m} \eta\right) \eta
$$

Da equaçào (5.152) concluimos que $h_{3}$ é uma quantidade positiva semidefinida, isto é

$$
\beta_{3} \geq 0 \text {. }
$$

Corn as equaçôes (5.147): (5.151) e (5.100), obtemos

$$
\alpha_{1} q_{i}^{T^{(2)}}+\alpha_{2} g_{i}^{(2)}=\frac{5}{2}\left(\frac{k}{m}\right)^{2} e T \frac{\partial T}{\partial x_{i}}+\frac{\frac{k}{m} T \lambda^{T}}{\frac{3}{2} \frac{k}{m}+E_{T}^{I}} \frac{\partial^{2} \eta_{k}}{\partial x_{i} \partial x_{k}}-\frac{5}{2} \frac{k}{m} T \eta_{v} \frac{\partial^{2} v_{k}}{\partial x_{i} \partial x_{k}}-2 \frac{k}{m} T \eta \frac{\partial^{2} v_{<i}}{\partial x_{j} \partial x_{j\rangle}}
$$

Da mesma forma, das equaçôs $(5.141),(5.147),(5.157)$ e (5.161), resulta

$$
u_{q_{i}}^{r(2)}+\chi_{2} g_{i}^{(2)}=e \frac{k}{m} T \epsilon_{T}^{J} \frac{\partial T}{\partial x_{i}}+\left(\frac{3 k}{2} \frac{k}{m} \eta_{v}+\frac{\frac{k}{m} T \lambda^{l}}{\frac{3}{2} \frac{k}{m}+\varepsilon_{T}^{l}}\right) \frac{\partial^{2} v_{k}}{\partial x_{i} \partial x_{k}} .
$$

Ao resolvermos o sistema de equaçös $(5.169)$ e $(5.170)$, obtemos para o fluxo de calor translacional

$$
\begin{aligned}
& q_{i}^{r^{(2)}}=\frac{\frac{k}{m} T}{\left(\alpha_{1} \lambda_{2}-\alpha_{2} \lambda_{11}\right)}\left\{\frac{g}{T}\left(\frac{5}{2} \frac{k}{m} T_{\lambda z}-\varepsilon_{T}^{t} \alpha_{2}\right) \frac{\partial T}{\partial x_{i}}-2 \eta \times z \frac{\partial^{2} v_{c i}}{\partial x_{j} \partial x_{2\rangle}}\right. \\
& \left.+\left[\left(\frac{\lambda^{T}}{\frac{3}{2} \frac{k}{m}+\varepsilon_{\tau}^{l}}-\frac{5}{2} \eta_{v}\right) x_{2}-\left(\frac{\lambda^{I}}{\frac{3}{2} \frac{k}{m_{3}}+\varepsilon_{r}^{l}}+\frac{3}{2} \eta_{\mathrm{v}}\right) \alpha_{2}\right] \frac{\partial^{2} v_{k}}{\partial r_{1} \partial x_{k}}\right\}
\end{aligned}
$$


e para o fluxo de energia da variável interna

$$
\begin{aligned}
& g_{i}^{(2)}=\frac{\frac{k}{m} T}{\left(\alpha_{2} \chi_{1}-\alpha_{1} \chi_{2}\right)}\left\{e\left(\frac{5}{2} \frac{k}{m} \chi_{1}-z_{T}^{t} \alpha_{1}\right) \frac{\partial T}{\partial x_{i}}-2 \eta \chi_{1} \frac{\partial^{2} v_{<i}}{\partial x_{j} \partial x_{j\rangle}}\right.
\end{aligned}
$$

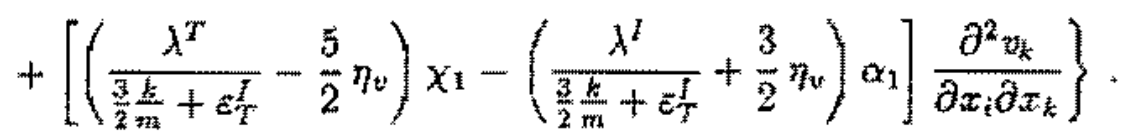

0 fluxo de calor total $q_{i}{ }^{(2)}$ é dado pela adiçào to termo que representa o fluxo de calor translacional $a_{i}^{T^{(2)}} \mathrm{com} 0$ termo que representa o fuxo de energia da variavel interna $g_{i}^{(2)}$, on seja

$$
q_{i}^{(2)}=q_{i}^{(2)}+g_{i}^{(2)}
$$

e com as equaçòes (5.17) ) (5.172), obtemos

$$
q_{i}^{(2)}=-\left(\lambda^{T}+\lambda^{t}\right) \frac{\partial T^{x}}{\partial x_{i}}+\gamma_{1} \frac{\partial^{2} v_{<i}}{\partial x_{j} \partial x_{j\rangle}}-\gamma_{i} \frac{\partial^{2} \eta_{j}}{\partial x_{i} \partial x_{j}}
$$

onde

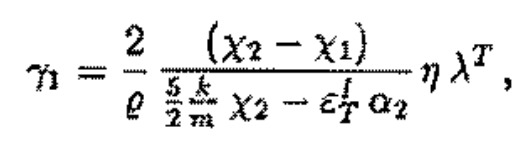

e

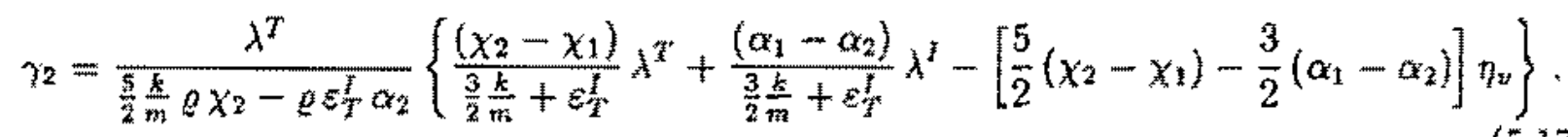

Através da equaçăo (5.123) podemos escrever para o tensor pressão

$$
p_{i j}^{(n)}=\frac{1}{3} p_{\tau \tau}^{(2)} \delta_{i j}+p_{\langle i, j\rangle}^{(2)}=\left(\frac{k}{n} T+\Pi^{(2)}\right) \delta_{i j}+p_{\langle i j\rangle}^{(2)}
$$

e, com as equacoes $(5.162)$ e $(5.165)$, que

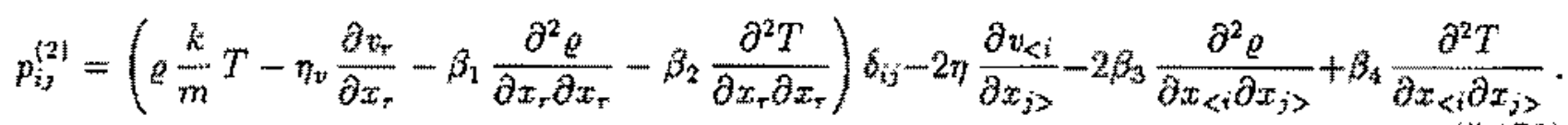

A equaçáo (5.174) para o fluxo de calor $q^{(2)}$ e a equagäo (5.178) para o tensor pressăo constituem as equaçoses linearizadas de Burnett para um gás poliatomico com umán varíavel interna: elas sào analogas às equaçoes $(5.74)$ e (5.67) obtidas através da teoria cinếtica. 


\subsubsection{Densidade e Fluxo de Entropia}

Para urn gás poliatômico com uma variável interna a densidade de entropia $\varrho s$ e ofuxo de entropia $\phi_{i}$ sâo dados, respectivamente, pelas equaçōes (5.113) e (5.114). Se reescrevermos estas equaços en ternos das variáveis temperatura $T$ e pressấo dinâmica II através das equaçòes $(3.124)$ e $(3.125)$, obtemos, respectivamente

$$
\begin{aligned}
& e s=e\left[\frac{k}{m} \ln \left(\frac{T^{3 / 2}}{e}\right)+\int \frac{e_{T}^{l}}{T} d T+a_{1}\right]-\frac{m}{k} n \int \frac{\varepsilon_{T T}^{l}}{T} d T \\
& -\frac{m}{k^{k T^{2}}}\left[\frac{3}{4}\left(1+\frac{3}{2} \frac{k}{m} \frac{1}{\varepsilon_{T}^{k}}\right)-\frac{T^{2}}{2} \frac{m}{k} \int \frac{\varepsilon_{T}^{J}}{T^{2}} d n\right] \mathrm{H}^{2}
\end{aligned}
$$

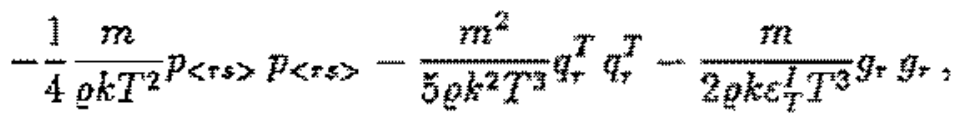

e

$$
\phi_{i}=\frac{1}{T}\left(q_{i}^{T}+g_{i}\right)+\frac{m}{g k T^{2}}\left(\frac{3}{2} \frac{k}{m} \frac{1}{\varepsilon_{T}^{T}} g_{i}-q_{i}^{T}\right) \Pi-\frac{2}{5} \frac{m}{g k T^{2}} p_{<j}>q_{j}^{T}
$$

onde $\varepsilon_{T T}^{I}=\frac{\partial e_{T}^{L}}{\partial T}$.

Algumas observações podem ser feitas à respeito das equaçōes para es e $\phi_{i}$. Por exemplo, no limite clássico. quando a variável interna está totalmente excitada decorre da equaçăo (5.118). que o calor especifico volume constante toma-se

$$
e_{T}^{t}=\left(\frac{\partial t^{T}}{\partial T}\right)_{e}=\text { constante }
$$

de modo que o termo linear em n năo aparece ná equazăo (5.179).

Se associarmos a variavel interna graus de liberdade rotacionais clássicos para a molécula do gás a energía interna especifica pode ser escrita, através do princípio da equipartiçäo da energia, como

$$
E^{l}(T)=\frac{3}{2} \frac{h}{m} T
$$

de modo que o calor especifico a volume constante é dado por

$$
e_{T}=\frac{3 k}{2} \frac{k}{m}
$$

e $\varepsilon_{T T}^{I}=0$. Desta forma, a equaçäo $(5.179)$ se reduz a:

$$
\begin{gathered}
p s=0\left[\frac{k}{m} \ln \left(\frac{T^{3}}{\ell}\right)+a_{1}\right]-\frac{3}{2} \frac{m}{\varrho k T^{2}} \Pi^{2}-\frac{1}{4} \frac{m}{\varrho k T^{2}} p_{\langle r s\rangle} p_{\langle r s\rangle} \\
-\frac{m^{2}}{\varrho^{2} T^{3}}\left(\frac{1}{5} q_{r}^{T} q_{T}^{T}+\frac{1}{3} q_{s}^{R} q_{s}^{R}\right)
\end{gathered}
$$


e para ofluxo de entropicatamos

$$
\phi_{i}=\frac{1}{T}\left(q_{i}^{T}+q_{i}^{n}\right)+\frac{m}{p^{k} T^{2}}\left(q_{i}^{n}-q_{i}^{T}\right) \Pi-\frac{2}{5} \frac{m}{e^{k T^{2}}} q_{j}^{T} p_{\langle i j\rangle}
$$

Nas duas ultimas equaçoes identificamos o fuxo de energia da variável interna com o fuxo de calor rotacional. isto $g_{i}=q_{i}^{R}$.

As equaçò $(3.184)$ e (5.185) são idênticas às que săo obtidas ao calcularmos a densidade de entropia e o fuxo de entropia na teoria cinêtica, equaçoes (3.128) e (3.130), através das integraçòes

$$
p s=-k \int f \ln f d \mathbf{c} d \omega
$$

e

$$
\phi_{i}=-k \int C_{i} f \ln f d c d \omega
$$

com a constante $a_{1}$ dada por

$$
a_{1}=3-\ln \left[\frac{1}{2 \pi k}\left(\frac{I}{m}\right)^{1 / 2}\right]^{3}
$$

\subsubsection{As Temperaturas $\vartheta$ e $T$ como Variáveis Termodinâmicas}

L'mâ parede ideal é caracterizada por nào participar ativamente dos processos em estudo. Em particular, ela năo armazena ou produz entropia, de modo que o fuxo de entropia de um dos lados da parede igual ao fluxo de entropia do lado oposto.

Se $t_{i}$ e um versor normal ă parede, podemos escrever que [veja equaçäo (E.18)]

$$
\left\|\phi_{i}\right\| e_{i}=0
$$

onde os colchetes indicam diferença da grandeza $\hat{h}_{i}$ em ambos os lados da parede.

Além disso. uma parede termométrica não armazena energia de modo que ofluxo de calor lotal $q_{i}=h_{i}^{7}+g_{i}$ numa direção normal à parede é, também, continuo, ou seja [veja equaçäo (4. 19$)$ :

$$
\text { lat } \epsilon_{i}=0
$$

Temperatura é uma variável adequada em termodinảmica desde que seja considerada ser facilnente mensurável e, em condições de equilbrio, seja conthutatravés de uma parede Lermométrica. Vamos analisar o que ocorre numa situagäo próxima do equilibrio.

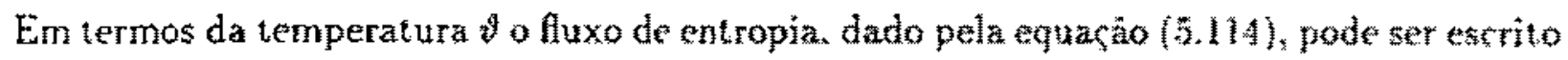
contho

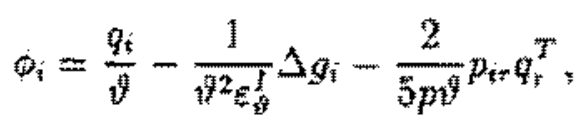


que, com as condiços dadas pelas equações $(5.189)$ e $(5.190)$, resulta em

$$
\left[\frac{1}{3}\right] q_{i} \epsilon_{i}=\llbracket\left[\frac{1}{\eta^{2} \varepsilon_{i}^{l}} \Delta g_{i}\right] \varepsilon_{i}+\left[\frac{2}{5 p^{0}} p^{p}<i r>q_{r}^{T}\right] \epsilon_{i}
$$

Em termos da temperatura $T$ temos, da equaçäo (5.180)

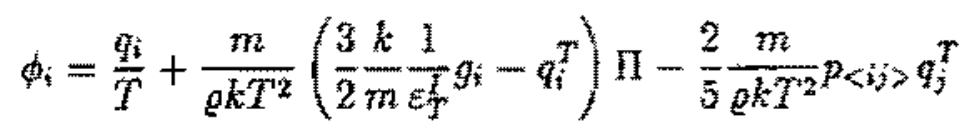

e, de forma análoga à obtenção da (5.192), temos:

$$
\llbracket \frac{1}{T} \rrbracket q_{i} e_{i}=\llbracket \frac{m}{\varrho k T^{2}}\left(q_{i}^{T}-\frac{3 k}{2} \frac{1}{m_{i}} \frac{1}{\varepsilon_{T}^{T}} q_{i}\right) \Pi \rrbracket e_{i}+\llbracket \frac{2}{5} \frac{m}{\varrho k T^{2}} p_{\langle i j\rangle} q_{i}^{T} \rrbracket e_{i}
$$

Das equaçós $(5.192)$ e (5.194) observamos que em processos que ocorrem próximo do equilibrio os termos nảo lineares nos campos poden ser negligenciados e as temperaturas $t$ e $T$ säo contínuas. No entanto, como foi observado em [26], a temperatura (e também $T$ ) perde muito do seu valor como variável termodinamica em processos nos quais os termos não lineares säo relevantes pois tanto $\downarrow$ quanto $T$ dexam de ser contínas em paredes idealizadas.

\subsubsection{Coeficientes de Transporte das Equações Linearizadas de Burnett}

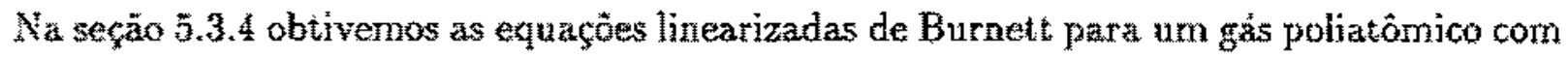
uma surável interna, através de uma teoria fenomenológica. Para o tensor pressào obtivernos a equaçä̃o $(5.178)$

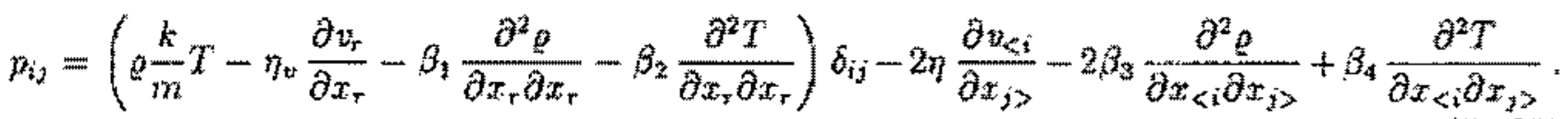

com as coeficientes $B_{i}$ dados respectivamente, pelas equaçöes $(5.164),(5.165),(5.167)$ e $(5.163)$ :

$$
\begin{gathered}
\beta_{1}=\frac{3}{2} \frac{\frac{3}{2} \frac{k}{m}+\varepsilon_{T}^{I}}{\varepsilon_{T}^{I}} \frac{\eta_{w}{ }^{2}}{\varrho^{2}} \\
\beta_{2}=\frac{m}{g k T}\left[\frac{\left.3 k \frac{\lambda^{I}}{2 m} \frac{\varepsilon_{T}^{I}+\frac{3}{3} \frac{k}{m}}{\varepsilon_{T}^{I}} \eta_{v}-\lambda^{T}\right] \eta_{w}}{\varepsilon_{T}^{I}}\right. \\
\beta_{3}=\frac{\eta^{2}}{\varrho^{2}}
\end{gathered}
$$




$$
\beta_{4}=\frac{4}{5} \frac{m}{0 k T}\left(\lambda^{T}-\frac{5 k}{2} \frac{k}{m}\right) \eta
$$

Além disso, conclumos que os coeficientes $\beta_{1}$ en $h_{3}$ sâ positivos semidefinidos, enquanto que os sinais dos coeficientes $\beta_{2}$ e $\beta_{4}$ permanecem indeterminados.

Para o vetor fuxo de calor obtivemos da equaça (5.174):

$$
q_{i}=-\left(\lambda^{x}+\lambda^{I}\right) \frac{\partial r}{\partial x_{i}}+\gamma_{1} \frac{\partial^{2} v_{<i}}{\partial x_{j} \partial x_{j\rangle}}-\gamma_{2} \frac{\partial^{2} v_{j}}{\partial x_{j} \partial x_{j}}
$$

com os coeficientes $\gamma_{i}$ dados respectivamente, pelas equaçóes $(5.176)$ e $(5.177)$ :

$$
\gamma_{1}=\frac{2}{\left.g \frac{5}{2} \frac{k}{m} x_{2}-\chi_{1}\right)} \eta_{\frac{1}{2} \alpha_{2}} \lambda^{T}
$$

e

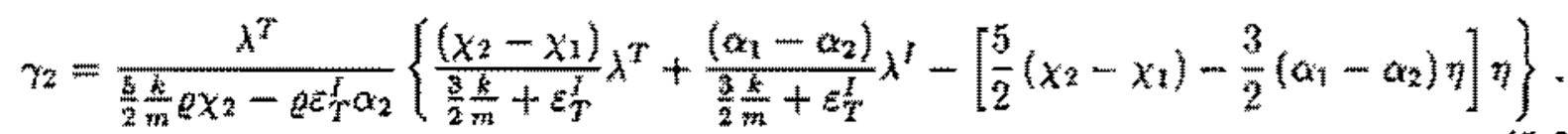

sendo que os sinais dos coeficientes de transporte $\gamma_{1}$ e $\gamma_{2}$ nầ estäo determinados.

Com o objetivo de confrontar os resultados da teoria fenomenológica com aqueles obtidos na seça õ. 2.3 através da teoria cinética, consideremos o caso de um gás políatónico de esferas rugosas. Desta forma, identificamos

$$
\begin{aligned}
\varepsilon^{k}(T) & =\frac{3}{2} \frac{k}{m} T^{T} \\
g_{i} & =g_{i}^{R}
\end{aligned}
$$

e

$$
\lambda^{y}=\lambda^{R}
$$

A equą̧âo (5.203) exprime o fato que a energia especifica interna e identificada com a energia especific clássica associada aos tres graus de liberdade rotacionais da molécula: na equaçầ (5.204) identificamos o fluxo de energia da variável interna com o fluxo de calor rotacional e na equação (5.205) igualamos o coefétente de condutividade tẻmica, associado à variável interna. an coeficiente de condutividade testrica rotacional.

Da equaçâo (5.204) temos para o calor específico rotacional a volume constante:

$$
E_{T}^{t}=\left(\frac{\partial \varepsilon^{T}}{\partial T}\right)_{s}=\frac{3}{2 m}
$$


Substituindo as equaçōes (5.203), (5.205) e (5.206) em (5.196) a (5.199), obtemos os coeficientes de transporte associados ao tensor pressão $p_{i j}$ :

$$
\begin{gathered}
\beta_{1}=3\left(\frac{\eta_{v}}{\varrho}\right)^{2}, \\
\beta_{2}=\frac{m}{\varrho k T}\left(\lambda^{R}-\lambda^{T}+\frac{3}{2} \frac{k}{m} \eta_{v}\right) \eta_{v}, \\
\beta_{3}=\left(\frac{\eta}{\varrho}\right)^{2}
\end{gathered}
$$

e

$$
\beta_{4}=\frac{4}{5} \frac{m}{\varrho k T}\left(\lambda^{T}-\frac{5}{2} \frac{k}{m} \eta\right) \eta .
$$

que são idênticos aos dados pelas equaçôes (5.68) obtidas através da teoria cinética.

Das equaçōes (5.201) e (5.203), resulta

$$
\gamma_{1}=4 \frac{m}{\varrho k}\left(\frac{\chi_{2}-\chi_{1}}{5 \chi_{2}-3 \alpha_{2}}\right) \eta \lambda^{T},
$$

e comparando a equação anterior com a (5.75), concluimos que

$$
\frac{\chi_{2}-\chi_{1}}{5 \chi_{2}-3 \alpha_{2}}=\frac{1}{5}
$$

ou seja

$$
\gamma_{1}=\frac{4}{5} \frac{m}{k \varrho} \eta \lambda^{T} .
$$

Das equaçōes (5.202), (5.205), (5.206) e (5.212), obtemos

$$
\gamma_{2}=\frac{2}{15}\left(\frac{m}{k}\right)^{2} \frac{\lambda^{T}}{\varrho}\left\{\lambda^{T}+\frac{5\left(\alpha_{1}-\alpha_{2}\right)}{\left(5 \chi_{2}-3 \alpha_{2}\right)} \lambda^{R}-\frac{15}{2} \frac{k}{m}\left[1-\frac{3\left(\alpha_{1}-\alpha_{2}\right)}{\left(5 \chi_{2}-3 \alpha_{2}\right)}\right] \eta_{v}\right\},
$$

e comparando com a equação (5.76), concluimos que

$$
\frac{\alpha_{1}-\alpha_{2}}{5 \chi_{2}-3 \alpha_{2}}=\frac{4}{75} \frac{(19 \kappa+3)}{(2 \kappa+1)(\kappa+1)},
$$

ou seja

$$
\gamma_{2}=\frac{2}{5}\left(\frac{m}{k}\right)^{2} \frac{\lambda^{T}}{\varrho}\left\{\lambda^{T}+\frac{4}{15} \frac{(19 \kappa+3)}{(2 \kappa+1)(\kappa+1)} \lambda^{R}-\frac{3}{10} \frac{k}{m} \frac{\left(50 \kappa^{2}-\kappa+13\right)}{(2 \kappa+1)(\kappa+1)} \eta_{v}\right\} .
$$

Verificamos assim que os coeficientes de transporte das equaçoos linearizadas de Burnett (5.195) e (5.200). obtidas da teoria fenomenológica e particularizadas para um gás poliatómico de esferas rugosas, podem ser determinados se recorrermos aos resultados obtidos através da teoria cinética. 


\subsection{Conclusões}

Neste capitulo determinamos as equaçỏes lineariadas de Burnet de duas maneiras distin* tas: através da teoria cinética dos gases e segundo uma teoria fenomenológica.

$\mathrm{Na}$ formulação da teoria cinética com cinco campos básicos (densidade de massa, velocidade e temperatura) empregamos terceira aproximação para a funçäo de distribuição com o método de Chapman-Enskogi assim, obtivemos as equaçós constitutivas para o tensor pressão $p_{i j}$ e para o vetor fluxo de calor $q_{i}$. As moleculas foram tratadas como esferas rigitas elásticas e rugosas, segundo o modelo de Bryan.

A equacắo para o tensor pressäo apresenta très termos que näo ocorrem para os gases monoatômicos. Un deles é bem conhecido sendo proporcional ao divergente da velocidade do gás tendo como coeficiente a velocidade volumétrica. Os outros dois säo proporcionais, respectivamente, ao laplaciano da densidade, denominado pressäo de densidade, e ao laplaciano da temperatura, denominado de pressão térmica.

$O$ vetor fluxo de calor também apresenta termos adicionais ao termo usual contendo o gradiente de temperatura obtido com a segunda aproximaçăo para a funçẫo de distribuiçầo. Estes termos são propocionais a gradientes de segunda ordem da velocidade do gás.

Os seis coeficientes de transporte das equaçôes linearizadas de Burnett foram deteminados em termos dos coeficientes obtidos através da segunda aproximaçâá para a lunçäo de distribuição: os coeficientes de condutividade térmita translacional $\lambda^{T}$ e rotacional $\lambda^{R}$, 0 co. eficiente de viscosidade cisalhante $\eta$ e o coeficiente de viscositade volumétrica $\eta_{w}$ bem como das propriedades moleculares: massa $m$, dismetro a e momento de inéria 1 . Outro aspecto observado é que os coeficientes de transporte sâo inversamente proporcionais à densidade do gás: assim, eles desempenham um papal importante medida que consideramos gases mais rarefeilos.

Em teoria cinetica a temperatura $T$ é associada à energia específica interna total das moleculas, as quais são: a energiti interna especifica de translaçầ $\varepsilon^{T}$ e a energia interrat especifica de rotaça $\varepsilon^{R}$. Assim, obtenos a temperatura do gás calculando o valor médio da energia cinética total das moléculas. Em outras palavras, em teoria cinética temperatura ê uma medida da agitaça molecular do gás.

Com o modelo molecular empregato, isto é. moléculas esféricas com seis grâus de liberdade a aplicabilidade da teoria se restringe a gases poliatömicos constituido de moléculas que apre* sentam os momentos de inércia todos iguais (moléculas esféricas) e com a razäo entre os calores especificos $7=\frac{c_{g}}{c_{*}}=\frac{4}{3}$ (moléculas com seis graus de liberdade). Dentre os gases que se enquadram neste modelo estät o metano $\mathrm{CH}_{4}$, no intervalo $60 K<T<200 \mathrm{~K}$. o tetrafluoreto de carbono $\mathrm{CF}_{4}$ no intervalo $60 \mathrm{~K}<\mathrm{T}<80 \mathrm{~K}$ e o metano- $\mathrm{C}_{4} \mathrm{CD}_{4}$. no intervalo $80 \mathrm{~h}<\mathrm{T}<160 \mathrm{~A}$.

Para determinarmos as equaçoes linearizadas de Burnett através de una teoria fenome* 
nológica utilizamos os resultados da teorio termodinâmica estendida para gases poliatômicos desenvolvida por Kremer $[26,27]$.

Nesta teoria empregamos dezessete campos encalares: densidade de massa, velocidade. tensor pressão, fluxo de calor translacional, densidade de energia associada à uma variável interna o o fluxo de energia associado variavel interna.

Estes campos básicos da termodinâmica são soluçôes de um conjunto de dezessete equaçôes de balanço ou de conservação; para resolve-las utilizamos um método iterativo devido à Maxwell. Com a primeira iteraçäo obtemos a lei de Navier-Stokes para o tensor pressão e a lei de Fourier para o retor fluxo de calor; com a segunda iteração obtemos as equaçōes linearizadas de Burnett.

Os roeficierutes para as equaçōes de Burnett assim obtidos não estão completamente determinados mesmo que identifiquemos a energia da variável interna com a energia de rotaça ou de vibraçäo da molécula. A determinação destes coeficientes pode ser feita experimentalmente ou corn o auxilio dos resultados da teoria cinética.

Para determiná-los através da teoria cinética identificamos a variável interna da teoria fenomenológica com a velocidade angular da molécula. de modo que a energia especifica da variável interna coincide com a energia específica rotacional e o fluxo de energia da variável interna coincide com of fuxo de energia rotacional. No entanto, somente estas identificações não säo suficientes pois há algumas diferenças conceituajs entre alguns campos empregados nạs duas teorias como mostra a tabela 5.1. Por exemplo, na teoria cinética a temperatura é identificada com o valor médio da energia interna total da molécula enquanto na teoria fenomenológica ela e associatia ao traço do tensor pressão.

Para confrontar os resultados determinamos relaçoes entre grandezas que representam si" tuaços fora do equilibrio para as duas teorias. Desta forma, podemos reduzir os resulta. dos da teoria fenomenológica aos da teoria cinética e, assim, identificar completamente todos os coeficientes de transporte das equaços linearizadas de Burnett obtidas através da teoria fenomenológica. Além disso. as equaçôes obtidas para a densidade de entropia e para o lluxo de entropia coincidem, como esperado, com as equaçöes correspondentes obtidas através da teoria cinćtica.

Através da "condiçào de salto" numa parede termométrica discutimos o papel desempenha* do pelas temperaturas, tanto na teoria cinética quanto na teoria fenomenológica. Uma pareds. termométrica é um dispositivo idealizado (um termómetro ideal, por exemplo) no qual as componentes normais dos fuxos de calor e de entropia são continuos alraves da parede. Com estas condiçoes. concluimos que a temperatura contínua em processos que se desenvolvem próximo do equilibrio. isto é. em processos nos quats os termos näo lineares nos campos podem ser negligenciados. No entanto, para processos em que os temos näo linearss säo considerados a tonyeratura é desconthua alraves de uma parede tamometrica e perde nuito da sev valor 
como grandeza termodinẳnica fundamental.

Como já observamos anteriormente, o emprego do modelo molecular de esferas rugosas conduz a valores altos para os coeficientes de transporte de um gás poliatónico, quando confrontamos estes valores com os obtidos experimentalmente. No entanto, as equações linearizadas de Burnet, obtidas neste capitulo podem ser úteis como ponto de partida para a explicação de fenômenos que dependen de segundos gradientes dos campos básicos (densidade, velocidade temperatura) como o efeito Scott e a propagação de ondas em gases rarefeitos. 


\section{Apêndice A}

\section{Integrais em Teoria Cinética}

Em teoria cinética dos gases as seguintes integrais definidas são freqüentemente utilizadas:

$$
\int_{0}^{\infty} x^{n} e^{-\alpha x^{2}} d x=\frac{1}{2} \Gamma\left(\frac{n+1}{2}\right)\left(\frac{1}{\alpha}\right)^{\frac{n+1}{2}}
$$

onde of uma constante,

$$
\left\{\begin{array}{l}
\Gamma(n+1)=n \Gamma(n) \\
\Gamma(1)=1 \\
\Gamma\left(\frac{1}{2}\right)=\sqrt{\pi} \\
\Gamma\left(n+\frac{1}{2}\right)=\frac{(2 n-1) ! 1}{2^{n}} \sqrt{\pi}
\end{array}\right.
$$

é a funçào gama, e

$$
(2 n+1) ! n=(2 n+1)(2 n-1) \ldots 5 \cdot 3 \cdot 1=\frac{(2 n+1) !}{2_{n} n !}
$$

Outras infegrais empregadas em teoris cinetica dos gases são do tipo

$$
\int C_{i,} C_{i_{2}} \ldots C_{i_{N}} f_{M} d \mathrm{c}=\frac{e}{m}\left(\frac{m}{2 \pi k T}\right)^{\frac{3}{2}} \int C_{i_{1}} C_{i_{2}} \ldots C_{i_{N}} \mathrm{e}^{-\frac{m^{2}}{2 k T}} d \mathrm{c}
$$

A integral da equagâno (A.4) é nula sempre que o número de componentes de velocidades peculiares for impar. Se $N=2$ a integral (A.4) pode ser escrita como:

$$
\begin{gathered}
\int C_{i} C_{j} f_{M} d \mathrm{c}=-\frac{e}{m}\left(\frac{m}{2 m T}\right)^{\frac{3}{2}}\left(\frac{k T}{m}\right) \int C_{t} \frac{\partial e^{-\frac{m m^{2}}{2 x T}}}{\partial C_{j}} d \mathrm{c} \\
=-\frac{e}{m}\left(\frac{m}{2 \pi k T}\right)^{\frac{3}{2}}\left(\frac{k T}{m}\right) \iint \frac{\partial C_{i} e^{-\frac{m c^{2}}{2 k T}}}{\partial C_{j}} d \mathrm{c}-\int e^{\left.-\frac{m c^{2}}{2 k T} \delta_{i j} d \mathrm{c}\right] .}
\end{gathered}
$$

A primeira integral na última igualdade é nula poia a funçâo exponencial lende a zero para grandes valores de C. Consequientemente, a equaçào (A.5) se reduz a: 


$$
\int C_{i} C_{j} f_{M} d c=-\frac{\varrho}{m}\left(\frac{m}{2 \pi k T}\right)^{\frac{3}{3}}\left(\frac{k T}{m}\right) \delta_{i j} \int e^{-\frac{m C^{2}}{2 \pi T}} d c .
$$

Por outro lado, é fácil verificar através da equaçäo (A.l), que

$$
\int e^{-\frac{m c^{2}}{2 \hbar T}} d \mathrm{c}=\frac{1}{3} \frac{m}{k T} \int C^{2} e^{-\frac{m c^{2}}{2 k T}} d c
$$

Segue. portanto, das equações (A.6) e (A.7) que

$$
\int C_{i} C_{j} f_{M} d \mathbf{c}=\frac{1}{3} \delta_{i j} \int C^{2} f_{M} d \mathrm{c} .
$$

Da mesma forma para $N=4$, temos da equaçän (A.4)

$$
\begin{aligned}
& \int C_{i} C_{j} C_{k} C_{l} f_{M} d \mathbf{c}=-\frac{e}{m}\left(\frac{m}{2 \pi k T}\right)^{\frac{3}{2}}\left(\frac{k T}{m}\right) \int C_{i} C_{j} C_{k} \frac{\partial e^{-\frac{m c^{2}}{3 k T}}}{\partial C_{l}} d \mathbf{c} \\
& =\frac{\varrho}{m}\left(\frac{m}{2 \pi k T}\right)^{\frac{3}{2}}\left(\frac{k T}{m}\right) \int\left(\delta_{i l} C_{j} C_{k}+C_{i} \delta_{j} C_{k}+C_{i} C_{j} \delta_{k l}\right) e^{-\frac{m C^{2}}{2 k T}} d \mathbf{c}
\end{aligned}
$$

A equação (A.9) pode ser escrita como

$$
\begin{gathered}
\int C_{i} C_{j} C_{k} C_{i} f_{M} d \mathrm{c}=\frac{e}{m}\left(\frac{m}{2 \pi k T}\right)^{\frac{3}{2}}\left(\frac{k T}{m}\right) \frac{1}{3}\left(\delta_{i l} \delta_{j k}\right. \\
\left.+\delta_{\mathrm{i} k} \delta_{j i}+\delta_{i j} \delta_{k i}\right) \int C^{z} e^{-\frac{m C^{3}}{2 m T^{2}}} d \mathrm{c}
\end{gathered}
$$

ao utilizarmos a equaçäo (A.8). Como, segundo a equação $(A .1)$

$$
\int C^{2} e^{-\frac{m c^{2}}{2 k T}} d \mathbf{c}=\frac{1}{5} \frac{m}{k T} \int C^{4} e^{-\frac{m c^{2}}{2 k T}} d \mathbf{c},
$$

as equaçỏes $($ A.10) e (A.11) nos fornecerm que

$$
\int C_{i} C_{j} C_{k} C_{i} f_{M} d \mathrm{c}=\frac{1}{15}\left(\delta_{i l} \delta_{j k}+\delta_{i k} \delta_{j 1}+\delta_{i j} \delta_{k j}\right) \int C^{4} f_{M} d \mathrm{c}
$$

A seguir daremos o resultado final para $N=6$, pois este pode ser obtido aplicado o msmo procedimento acima:

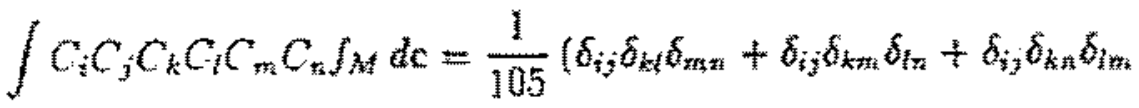

$$
\begin{aligned}
& +\delta_{i k} \delta_{j l} \delta_{m k}+\delta_{i k} \delta_{j m} \delta_{m n}+\delta_{i k} \delta_{j n} \delta_{l m}+\delta_{i k} \delta_{j k} \delta_{m n}+\delta_{i k} \delta_{j m} \delta_{k n n}
\end{aligned}
$$




$$
\begin{aligned}
+\delta_{i n} \delta_{j n} \delta_{k m} & +\delta_{i m} \delta_{j k} \delta_{m}+\delta_{i m} \delta_{j l} \delta_{k n}+\delta_{i m} \delta_{j n} \delta_{k l}+\delta_{i n} \delta_{j k} \delta_{l m} \\
& +\delta_{i n} \delta_{j l} \delta_{k m}+\delta_{k n} \delta_{j m} \delta_{k l} \int C^{6} \delta_{M} d \mathrm{c}
\end{aligned}
$$

As seguintes tómulas são válidas para $n=0,1,2,3, \cdots$ :

$$
\begin{aligned}
& \int(g \cdot k)^{n} d k=\frac{2 \pi}{n+1} g^{n} \\
& \int k_{i}(g \cdot \mathbf{k})^{*} d k=\frac{2 \pi}{n+2} g^{(n+1)} g_{i}
\end{aligned}
$$

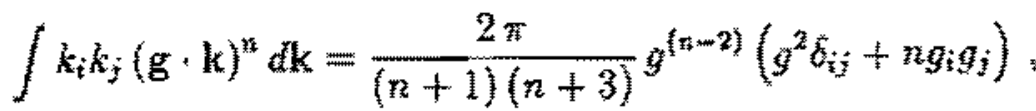

$$
\begin{aligned}
& \int k_{i} k_{j} k_{k}(\mathrm{~g} \cdot \mathrm{k})^{n} d \mathrm{k}=\frac{2 \pi}{(n+2)(n+4)} g^{(n-3)}\left[g^{2}\left(g_{i} \delta_{j k}+g_{j} \delta_{i k}+g_{k} \delta_{i j}\right)+(n-1) g_{i j} g_{j k}\right] \\
& \int k_{i} k_{j} k_{r} k_{s}(\mathrm{~g} \cdot \mathrm{k})^{n} \mathrm{k}=\frac{2 \pi}{(n+1)(n+3)(n+5)} g^{(n+4)}\left[g^{4}\left(\delta_{i j} \delta_{s}+\delta_{i r} b_{j k}+\delta_{i s} \delta_{j s}\right)\right. \\
& \left.+\pi g^{2}\left(g_{i} g_{j} \delta_{r s}+g_{i} g_{r} \delta_{j s}+g_{i} g_{s} \delta_{j}+g_{j} g_{r} \hat{b}_{s s}+g_{j} g_{s} \delta_{i r}+g_{r} g_{s} \delta_{i j}\right)+n(n-2) g_{i} g_{j} g_{s} g_{s}\right]= \\
& \int k_{i} k_{j} k_{k} k_{k} k_{m}(\mathrm{~g} \cdot \mathrm{k})^{n} d \mathrm{k}=\frac{2 \pi}{(n+2)(n+4)(n+6)} g^{(n-5)}\left\{g ^ { 4 } \left[g_{i}\left(\delta_{j k} \delta_{l m}+\delta_{j l} \delta_{k m}+\delta_{j m} \delta_{k l}\right)\right.\right.
\end{aligned}
$$

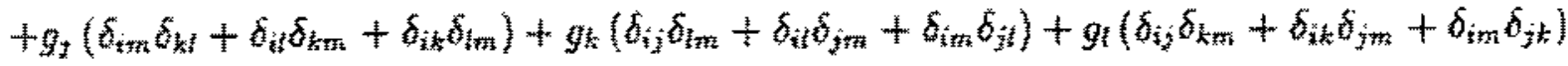

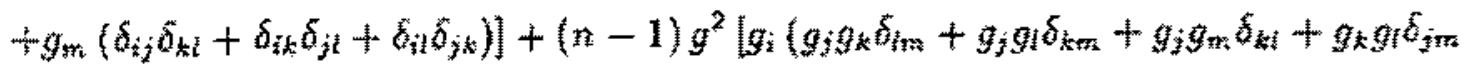

$$
\begin{aligned}
& \left.\left.+g_{k} g_{m} \delta_{i j}+g_{l} g_{m} \delta_{j k}\right)+g_{k} g_{t} g_{m} \delta_{i j}+g_{j} g_{i} g_{m} \delta_{i k}+g_{j} g_{k} g_{m} \delta_{i l}+g_{j} g_{k} g_{l} \delta_{i m}\right] \\
& \left.+(n-1)(n-3) g_{i} g_{j} g_{k} g_{1} g_{m}\right\} \text {. }
\end{aligned}
$$

Para provar as equaçoes (A.14) a (A.19), consideremos que o elemento de ângulo sólido dk pode ser escrito como:

$$
d \mathrm{k}=\operatorname{sen} 0 \mathrm{~d} n \mathrm{a} E
$$

onde $\theta$ e $\varepsilon$ săo ângulos polares de $\mathrm{k}_{\mathrm{r}} \mathrm{e}$

$$
k_{i}=\left(\begin{array}{r}
\cos \operatorname{sen} \theta \\
\operatorname{sen} \operatorname{sen} \theta \\
\cos \theta
\end{array}\right)
$$

sendo $0 \leq 0 \leq \frac{\pi}{2}$ e $0 \leq \varepsilon \leq 2 \pi$ 
Por outro lado e sem perda de generalidade, se for orientado na direçäo do eixo $x_{3}$ temos que $(\mathrm{g} \cdot \mathrm{k})=g \cos \theta$.

Para provarmos a relaçăo (A.17) escolhemos dois vetores constantes a e b que não dependem de $\theta$ e. Ao multiplicarmos escalarmente o lado esquerdo da equą̧̧o (A.17) e integrando a equação resltante, obtemos:

$$
\begin{aligned}
& \int(\mathbf{a} \cdot \mathbf{k})(\mathbf{b} \cdot \mathbf{k})(\mathrm{g} \cdot \mathbf{k})^{*} k_{i} d \mathbf{k} \\
& =\int\left(a_{x} \operatorname{sen} \theta \cos \epsilon+a_{y} \operatorname{sen} \theta \operatorname{sen} \varepsilon+a_{z} \cos \theta\right)\left(b_{x} \operatorname{sen} \theta \cos \varepsilon+b_{y} \operatorname{sen} \theta \operatorname{sen} \varepsilon+b_{z} \cos \theta\right) \\
& \times\left(\begin{array}{c}
\cos \varepsilon \operatorname{sen} \theta \\
\operatorname{sen} \varepsilon \operatorname{sen} \theta \\
\cos \theta
\end{array}\right) g_{i}^{\mathrm{n}} \cos ^{\mathrm{n}} \theta \operatorname{sen} \theta d \theta d \varepsilon \\
& =\frac{2 m}{(n+2)(n+4)} g^{n}\left\{\left[a_{z} b_{x}+a_{y} b_{y}+(n+2) a_{z} b_{z}\right]\left(\begin{array}{l}
0 \\
0 \\
1
\end{array}\right)_{i}+\left(a_{z} b_{x}+a_{x} b_{z}\right)\left(\begin{array}{l}
1 \\
0 \\
0
\end{array}\right)_{i}\right. \\
& \left.+\left(a_{y} b_{z}+a_{z} b_{y}\right)\left(\begin{array}{l}
0 \\
1 \\
0
\end{array}\right){ }_{i}\right)=\frac{2 \pi}{(n+2)(n+4)} g^{n-3}\left[g_{z}(a \cdot b) g^{2}+b_{i}(a \cdot g) g^{2}\right. \\
& \left.+a_{i}(\mathrm{~b} \cdot \mathrm{g}) g^{2}+(n-1) g(\mathrm{a} \cdot \mathrm{g})(\mathrm{b} \cdot \mathrm{g})\right]
\end{aligned}
$$

A relaçăo (A.l T) é facilmente obtida com a equaçào anterior. 


\section{Apêndice B}

\section{Representações Tensoriais}

\section{B.1 Representação para o Tensor $H_{<i j>k}$}

Consideremas a funçäo tensorial isotrópica $H_{<i j\rangle} k$ do tensor autissimétrico $B_{i j}=\varepsilon_{i j} H_{k}$ e do vetor gradiente de temperatura $\frac{\partial T}{\partial x_{i}}: H_{k}$ são as componentes do vetor axial campo magnético.

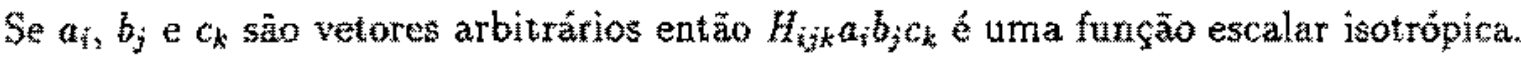

De acordo com os teoremas de represent.z̧ào para funçoes tensoriais [21] os invariantes escalares para uma função escalar isotrópica de um tensor antissimétrico $B_{i j ̧}$ e dos vetores $v_{a}$. $a=1,2, \cdots, n$ säo:

$$
v_{\alpha} \cdot v_{\alpha}, v_{\alpha} \cdot v_{0}, \operatorname{tr} B^{z}, v_{\alpha} \cdot B^{2} \cdot v_{\alpha}, v_{\alpha} \cdot B \cdot v_{b \beta} \text { e } v_{\alpha} \cdot B^{2} \cdot v_{b}
$$

onde $\alpha, \beta=1,2, \cdots, n$ e $\alpha<\beta$.

Se considerarmos o caso linear nos vetores $a_{i}, b_{j}, c_{k}$ e $d_{1}$ temos, da equaça $(B .1)$, os seguintes invariantes:

$$
\begin{aligned}
& \text { a } a b, \quad a \cdot c, \quad a \cdot d, \quad b \cdot c, \quad b \cdot d, \quad c \cdot d, \\
& \operatorname{tr} \mathbf{B}^{2}, \quad \mathbf{a} \cdot \mathbf{B} \cdot \mathbf{b}, \quad \mathbf{a} \cdot \mathbf{B} \cdot \mathbf{c}, \quad \mathbf{a} \cdot \mathbf{B} \cdot \mathbf{d}, \quad \mathbf{b} \cdot \mathbf{B} \cdot \mathbf{c}, \quad \mathbf{b} \cdot \mathbf{B} \cdot \mathbf{d}, \\
& c \cdot B \cdot d, \quad a \cdot B^{2} \cdot b, a \cdot B^{2} \cdot c, a \cdot B^{2} \cdot d, b \cdot B^{2} \cdot c, b \cdot B^{2} \cdot d, \\
& \mathrm{c} \cdot \mathrm{B}^{2} \cdot \mathrm{d} \text {, }
\end{aligned}
$$

onde

$$
\left(\mathbf{G}^{2}\right)_{i j}=B_{i m} B_{m j}=H_{i} H_{j}-H^{2} \delta_{i j}
$$

A funça escalar escrita como uma combinaçấ trilinear em a, b. c e linear em d, ou seja:

$$
\begin{gathered}
H_{r 3 t}\left(a_{t} b_{5} c_{t}=a_{1}(a \cdot b)(c \cdot d)+\alpha_{2}(a \cdot b)(c \cdot B \cdot d)+\alpha_{3}(a \cdot b)\left(c \cdot B^{2} \cdot d\right)+\alpha_{4}(a \cdot c)(b \cdot d)\right. \\
+\alpha_{5}(a \cdot c)(b \cdot B \cdot d)+\alpha_{6}(a \cdot c)\left(b \cdot B^{2} \cdot d\right)+\alpha_{7}(a \cdot d)(b \cdot c)+\alpha_{8}(a \cdot d)(b \cdot B \cdot c) \\
+\alpha_{9}(a \cdot d)\left(b \cdot B^{2} \cdot c\right)+\alpha_{10}(b \cdot c)(a \cdot B \cdot d)+a_{11}(b \cdot c)\left(a \cdot B^{2} \cdot d\right)
\end{gathered}
$$




$$
\begin{aligned}
& +\alpha_{12}(\mathbf{b} \cdot \mathbf{d})(\mathbf{a} \cdot \mathbf{B} \cdot \mathbf{c})+\alpha_{13}(\mathbf{b} \cdot \mathbf{d})\left(\mathbf{a} \cdot \mathbf{B}^{2} \cdot \mathbf{c}\right)+\alpha_{14}(\mathbf{c} \cdot \mathbf{d})(\mathbf{a} \cdot \mathbf{B} \cdot \mathbf{b})+\alpha_{15}(\mathbf{c} \cdot \mathbf{d})\left(\mathbf{a} \cdot \mathbf{B}^{2} \cdot \mathbf{b}\right) \\
& +\alpha_{13}(\mathbf{a} \cdot \mathbf{B} \cdot \mathbf{b})(\mathbf{c} \cdot \mathbf{B} \cdot \mathbf{d})+\alpha_{17}(\mathbf{a} \cdot \mathbf{B} \cdot \mathbf{b})\left(\mathbf{c} \cdot \mathbf{B}^{2} \cdot \mathbf{d}\right)+\alpha_{18}(\mathbf{a} \cdot \mathbf{B} \cdot \mathbf{c})(\mathbf{b} \cdot \mathbf{B} \cdot \mathbf{d})+ \\
& +\alpha_{19}(\mathbf{a} \cdot \mathbf{B} \cdot \mathbf{c})\left(\mathbf{b} \cdot \mathbf{B}^{2} \cdot \mathbf{d}\right)+\alpha_{20}(\mathbf{a} \cdot \mathbf{B} \cdot \mathbf{d})(\mathbf{b} \cdot \mathbf{B} \cdot \mathbf{c})+\alpha_{21}(\mathbf{a} \cdot \mathbf{B} \cdot \mathbf{d})\left(\mathbf{b} \cdot \mathbf{B}^{2} \cdot \mathbf{c}\right) \\
& +\alpha_{22}(\mathbf{b} \cdot \mathbf{B} \cdot \mathbf{c})\left(\mathbf{a} \cdot \mathbf{B}^{2} \cdot \mathbf{d}\right)+\alpha_{23}(\mathbf{b} \cdot \mathbf{B} \cdot \mathbf{d})\left(\mathbf{a} \cdot \mathbf{B}^{2} \cdot \mathbf{c}\right)+\alpha_{24}(\mathbf{c} \cdot \mathbf{B} \cdot \mathbf{d})\left(\mathbf{a} \cdot \mathbf{B}^{2} \cdot \mathbf{b}\right) \\
& +\alpha_{25}\left(\mathbf{a} \cdot \mathbf{B}^{2} \cdot \mathbf{b}\right)\left(\mathbf{c} \cdot \mathbf{B}^{2} \cdot \mathbf{d}\right)+\alpha_{26}\left(\mathbf{a} \cdot \mathbf{B}^{2} \cdot \mathbf{c}\right)\left(\mathbf{b} \cdot \mathbf{B}^{2} \cdot \mathbf{d}\right)+\alpha_{27}\left(\mathbf{a} \cdot \mathbf{B}^{2} \cdot \mathbf{d}\right)\left(\mathbf{b} \cdot \mathbf{B}^{2} \cdot \mathbf{c}\right),(\mathbf{B} \cdot 4)
\end{aligned}
$$

com os coeficientes $\alpha_{i}=\alpha_{i}\left(\operatorname{tr} \mathrm{B}^{2}\right)=\alpha_{i}\left(H^{2}\right)$.

Realizando derivaçoes parciats da equuaçă anterior em relaçăo as componentes vetoriais af, $b_{j}$ e c obtemos:

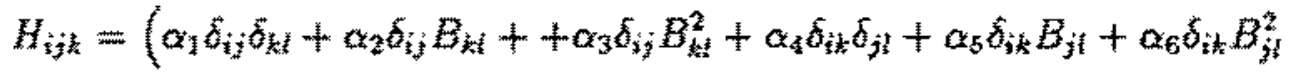

$$
\begin{aligned}
& +\alpha_{7} \delta_{i k} \delta_{j k}+\alpha_{8} \delta_{i l} B_{3 k}+\alpha_{5} \delta_{i l} B_{j k}^{z}+\alpha_{10} \delta_{j k} B_{i t}+\alpha_{11} \delta_{j k} B_{i j}^{2}+\alpha_{12} \delta_{j t} B_{i k} \\
& +\alpha_{13} \delta_{j i} B_{i k}^{2}+\alpha_{14} \delta_{k i} B_{i j}+\alpha_{15} \delta_{k i} B_{i j}^{2}+\alpha_{16} B_{i j} B_{k l}+\alpha_{17} B_{i j} B_{k l}^{2}+\alpha_{18} B_{i k} B_{j l} \\
& +\alpha_{19} B_{i k} B_{j k}^{2}+\alpha_{20} B_{i l} B_{j k}+\alpha_{21} B_{i l} B_{j k}^{z}+\alpha_{22} B_{j k} B_{i i}^{2}+\alpha_{23} B_{j l} B_{i k}^{2}+\alpha_{24} B_{i j}^{2} B_{k l}^{2} \\
& \left.+\alpha_{2 \xi} B_{i k}^{2} B_{j l}^{2}+\alpha_{26} B_{i l}^{2} B_{j l}^{2}\right) d_{l} \text {. }
\end{aligned}
$$

Se utilizarmos a equaçäo (B.3) e mantivermos apenas os termos linearmente independentes podemos escrever a equação anterior como:

$$
\begin{aligned}
& H_{i j k}=\left[\beta_{1}^{\prime} \delta_{i j} \delta_{k i}+\beta_{2}^{\prime} \delta_{i j} \varepsilon_{k i p} H_{p}+\beta_{3}^{\prime} \delta_{i j} H_{k} H_{i} \div \beta_{4}^{\prime}\left(\delta_{i k} \delta_{j i}+\delta_{i l} \delta_{j k}\right)\right. \\
& +\beta_{S}^{\prime}\left(\delta_{i k} \varepsilon_{j l p} H_{p}+\delta_{j k} \varepsilon_{i p} H_{\mathfrak{p}}\right)+\beta_{6}^{\prime}\left(\delta_{i t} \varepsilon_{j k_{p}} H_{p}+\delta_{j i} \varepsilon_{i k g} H_{p}\right) \\
& +\beta_{7}^{\prime}\left(\varepsilon_{i k p} \varepsilon_{j l q}+\varepsilon_{j k p} \varepsilon_{i q q}\right) H_{p} H_{q}+\beta_{8}^{\prime}\left(\delta_{i k} H_{j} H_{t}+\delta_{j k} H_{i} H_{i}\right) \\
& +\beta_{\mathrm{g}}^{2} \delta_{k i} H_{i} H_{j}+\beta_{\mathrm{ta}}^{2}\left(\epsilon_{i k_{p}} H_{i}+\varepsilon_{j k} H_{i}\right) H_{p} H_{i} \\
& \left.+\beta_{1 \mathrm{l}}^{t}\left(\varepsilon_{\mathrm{ip}} H_{j}+\varepsilon_{i t_{p}} H_{i}\right) H_{p} H_{k}+\beta_{12}^{\prime} H_{i} H_{i} H_{k} H_{1}\right] \frac{\partial T}{\partial x_{i}},
\end{aligned}
$$

onde identificamos $d_{t}=\frac{\partial T}{\partial x_{i}}$.

Estamos interessados na função tensorial $H_{<i\rangle\rangle k}$, simétrica e sem traço nos dois primeiros indices, dada por

$$
H_{<i j>k}=H_{i j k}-\frac{1}{3} H_{r k k} \delta_{i j}
$$

Assim. das equaçoes (B.6) e (B.7). podemos escrever que:

$$
H_{<i j>k}=\left[\beta_{1}\left(\delta_{i k} \delta_{j l}+\delta_{i l} \delta_{j k}-\frac{2}{3} \delta_{i j} \delta_{k l}\right)+\beta_{2}\left(\delta_{i k E_{j t p}}+\delta_{j k} \xi_{i l p}-\frac{2}{3} \delta_{i j} \varepsilon_{k l p}\right) H_{p}\right.
$$




$$
\begin{aligned}
& +\beta_{3}\left(\delta_{i k} \varepsilon_{j k p}+\delta_{j l \varepsilon_{i k p}}-\frac{2}{3} \delta_{i j} \varepsilon_{k l p}\right) H_{p}+\beta_{4}\left(\delta_{i k} H_{j}+\delta_{j k} H_{i}-\frac{2}{3} \delta_{i j} H_{k}\right) H_{l} \\
& +\beta_{5}\left(\delta_{i} H_{j}+\delta_{j i} H_{i}-\frac{2}{3} \delta_{i j} H_{l}\right) H_{k}+\beta_{G}\left(H_{i} H_{j}-\frac{1}{3} H^{2} \delta_{i j}\right) \delta_{k l} \\
& +\beta_{7}\left(\varepsilon_{i k_{p}} H_{j}+\varepsilon_{j k_{p}} H_{i}\right) H_{p} H_{i}+\beta_{8}\left(\varepsilon_{i p_{p}} H_{j}+\varepsilon_{i p p} H_{i}\right) H_{p} H_{k} \\
& \left.+\beta_{0}\left(H_{i} H_{i}-\frac{1}{3} H^{2} \delta_{i j}\right) H_{k} H_{i}+\beta_{10}\left(\delta_{i k} \delta_{j i}+\delta_{i l} \delta_{j k}-\frac{2}{3} \delta_{i j} \delta_{k l}\right)\right] \frac{\partial T}{\partial z_{i}} .
\end{aligned}
$$

Nesta equaçăo os coeficientes $\beta_{3}$ säo funçoes $\beta_{i}\left(H^{2}\right), i=2, \cdots, 10$, enquanto que o coeficiente $A_{1}$ independe de $H$; de fato, para $H=0$ a equaçz̃o $(B .8)$ se redu a

$$
H_{\langle i j\rangle k}=\hat{\sigma}_{1}\left(\delta_{i k} \delta_{j t}+\delta_{i t} \delta_{j k}-\frac{2}{3} \delta_{t j} \delta_{k t}\right) \frac{\partial T}{\partial t a t}
$$

que é a representação para o tensor $H_{<i j>k}$ como funcão somente do vetot $\frac{\partial T}{\partial x_{3}}$.

\section{B.2 Representação para um Tensor de Quarta Ordem}

Consideremos a função escalar isotrópica $A_{i j k} a_{i} b_{j} c_{k} d_{l}$ do vetores arbitrários $a_{i}, b_{j}, c_{k}$ e $d_{j}$ e do tensor antissimétrico $B_{i j}=\epsilon_{i j k} H_{k}$, onde $H_{k}$ säo as componentes do vetor axial campo magnético. Empregando teoremas de representaçäo para funçôes escalares isotrópicas [21] vèrificamos que os invariantes escalares para $A_{i j k j} a_{i} b_{j} c_{k} d_{l}$ sầo dados pela equação (B.2).

Se considerarmos o caso quadrilinear nos vetores $a_{i}, b_{j}, c_{k}$ e $d_{l}$, a função escalar pode ser escrita como:

$$
\begin{aligned}
& A_{i 2 k} a_{;} b_{3} c_{k} d_{1}=\beta_{1}(\mathrm{a} \cdot \mathrm{b})(\mathrm{c} \cdot \mathrm{d})+\beta_{3}(\mathrm{a} \cdot \mathrm{b})(\mathrm{c} \cdot \mathrm{B} \cdot \mathrm{d})+\beta_{\mathrm{s}}(\mathrm{a} \cdot \mathrm{b})\left(\mathrm{c} \cdot \mathrm{B}^{2} \cdot \mathrm{d}\right)+\beta_{4}(\mathrm{a} \cdot \mathrm{c})(\mathrm{b} \cdot \mathrm{d})
\end{aligned}
$$

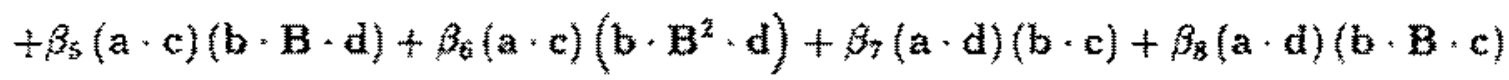

$$
\begin{aligned}
& +\beta_{9}(\mathrm{a} \cdot \mathrm{d})\left(\mathrm{b} \cdot \mathrm{B}^{2} \cdot \mathrm{c}\right)+\beta_{10}(\mathrm{~b} \cdot \mathrm{c})(\mathrm{a} \cdot \mathbf{B} \cdot \mathrm{d})+\beta_{11}(\mathrm{~b} \cdot \mathrm{c})\left(\mathrm{a} \cdot \mathbf{B}^{2} \cdot \mathrm{d}\right) \\
& +\beta_{12}(\mathbf{b} \cdot \mathbf{d})(\mathbf{a} \cdot \mathbf{B} \cdot \mathbf{c})+\beta_{13}(\mathbf{b} \cdot \mathbf{d})\left(\mathbf{a} \cdot \mathbf{B}^{2} \cdot \mathbf{c}\right)+\beta_{14}(\mathrm{c} \cdot \mathrm{d})(\mathbf{a} \cdot \mathbf{B} \cdot \mathbf{b})+\beta_{13}(\mathbf{c} \cdot d)\left(\mathbf{a} \cdot \mathbf{B}^{2} \cdot \mathbf{b}\right) \\
& +\beta_{16}(\mathbf{a} \cdot \mathbf{B} \cdot \mathbf{b})(\mathrm{c} \cdot \mathbf{B} \cdot \mathbf{d})+\beta_{17}(\mathbf{a} \cdot \mathbf{B} \cdot \mathbf{b})\left(\mathbf{c} \cdot \mathbf{B}^{2} \cdot \mathbf{d}\right)+\beta_{1 s}(\mathbf{a} \cdot \mathbf{B} \cdot \mathbf{c})(\mathbf{b} \cdot \mathbf{B} \cdot \mathrm{d}) \\
& +\beta_{19}(\mathbf{a} \cdot \mathbf{B} \cdot \mathrm{c})\left(\mathrm{b} \cdot \mathbf{B}^{2} \cdot \mathrm{d}\right)+\beta_{20}(\mathrm{a} \cdot \mathbf{B} \cdot \mathrm{d})(\mathrm{b} \cdot \mathbf{B} \cdot \mathbf{c})+\beta_{2 \mathrm{I}}(\mathrm{a} \cdot \mathbf{B} \cdot \mathrm{d})\left(\mathbf{b} \cdot \mathbf{B}^{2} \cdot \mathrm{c}\right) \\
& +\beta_{22}(\mathrm{~b} \cdot \mathrm{B} \cdot \mathrm{c})\left(\mathrm{a} \cdot \mathrm{B}^{2} \cdot \mathrm{d}\right)+\beta_{23}(\mathrm{~b} \cdot \mathbf{B} \cdot \mathbf{d})\left(\mathbf{a} \cdot \mathbf{B}^{2} \cdot \mathbf{c}\right)+\beta_{24}(\mathbf{c} \cdot \mathbf{B} \cdot \mathrm{d})\left(\mathbf{a} \cdot \mathbf{B}^{2} \cdot \mathbf{b}\right) \\
& +\beta_{2 s}\left(\mathrm{a} \cdot \mathrm{B}^{2} \cdot \mathrm{b}\right)\left(\mathrm{c} \cdot \mathrm{B}^{2} \cdot \mathrm{d}\right)+\beta_{26}\left(\mathbf{a} \cdot \mathbf{B}^{2} \cdot \mathrm{c}\right)\left(\mathrm{b} \cdot \mathbf{B}^{2} \cdot \mathrm{d}\right)+\beta_{22}\left(\mathrm{a} \cdot \mathbf{B}^{2} \cdot \mathrm{d}\right)\left(\mathbf{b} \cdot \mathbf{B}^{2} \cdot \mathrm{c}\right) \cdot(\mathbf{B} \cdot 10)
\end{aligned}
$$

con os coeficientes $\beta_{i}=\beta_{i}\left(\operatorname{tr} \mathbf{B}^{2}\right)=\beta_{i}\left(H^{2}\right)$. 
Ao realizarmos as derivaçố da equação anterior em relação às componentes vetoriais $a_{x}$, $b_{j,} c_{k,} d_{j}$ e considerarmos o tensor $A_{i j k l}$ simétrico nos dois primeiros e nos dois últimos índices, isto é

$$
A_{i j k l}=A_{j k k l} \text { e } A_{i j k l}=A_{i j k k}
$$

obtemos com o emprego da equaçäo (B.3):

$$
\begin{aligned}
& A_{i j k l}=\alpha_{1}^{\prime} \delta_{i j} \delta_{k l}+\alpha_{2}^{\prime}\left(\delta_{i k} \delta_{j l}+\delta_{i l} \delta_{j k}\right)+\alpha_{3}^{\prime} \delta_{i j} H_{k} H_{l}+\alpha_{4}^{\prime} \delta_{k l} H_{i} H_{j}
\end{aligned}
$$

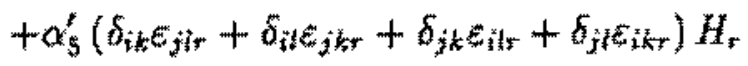

$$
\begin{aligned}
& +\alpha_{6}^{\prime}\left(\delta_{i k} H_{j} H_{1}+\delta_{i 1} H_{j} H_{k}+\delta_{j} H_{i} H_{3}+\delta_{j i} H_{i} H_{k}\right)
\end{aligned}
$$

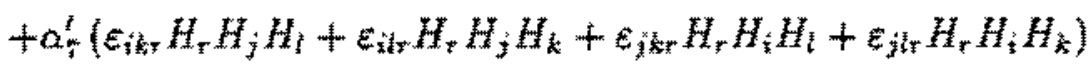

$$
\begin{aligned}
& \alpha_{3}^{\prime} H_{i} H_{j} H_{*} H_{i}
\end{aligned}
$$

A parte simétrica e sem traço nos dois primeiros e nos dois últimos indices é defnida por

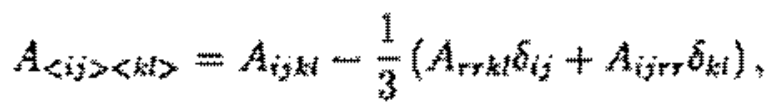

e da equaçào (B.12) temos:

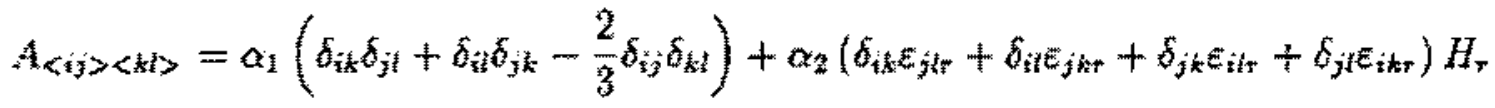

$$
\begin{aligned}
& +\alpha_{3}\left(\delta_{i k} H_{j} H_{i}+\delta_{i j} H_{j} H_{k}+\delta_{j k} H_{i} H_{i}+\delta_{j i} H_{i} H_{k}-\frac{4}{3} \delta_{k i} H_{i} H_{j}-\frac{4}{3} \delta_{i j} H_{k} H_{l}+\frac{4}{9} H^{2} \delta_{i j} \delta_{k l}\right) \\
& +a_{4}\left(\varepsilon_{i k r} H_{j} H_{l}+\varepsilon_{i t r} H_{j} H_{k}+\varepsilon_{j k r} H_{i} H_{1}+\varepsilon_{j t r} H_{i} H_{k}\right) H_{r} \\
& +\alpha_{5}\left(H_{i} H_{j} H_{k} H_{l}-\frac{1}{3} H^{2} \delta_{k i} H_{i} H_{j}-\frac{1}{3} \delta_{i j} H^{2} H_{k} H_{l}+\frac{1}{9} H^{4} \delta_{i j} \delta_{k l}\right) \text {. }
\end{aligned}
$$

que a representaçăo para um tensor de quarta ordem, simétrico e sem traço nos dois primeiros e nos dois ultimos indices.

\section{B.3 Inversão de um Tensor de Segunda Ordem}

Lm tensor de segunda ordem (S) ig soluçăo da seguinte equação, dada através do teorema de Cayley Hamilion:

$$
\left(\mathrm{B}^{3}\right)_{i m}-I_{1}\left(\mathrm{~S}^{2}\right)_{i m}+I_{2}(S)_{i m}-I_{3} \delta_{i m}=0
$$

onde

$$
I_{1}=\mathfrak{S}_{3 i}, \quad I_{2}=\frac{1}{2}\left[\left(S_{i i}\right)^{2}-\left(S^{2}\right)_{i i}\right] \text { e } I_{3}=\operatorname{det}(S)
$$


Se multiplicarmos a equaçăo (B.15) pelo tensor inverso $\left(\mathrm{S}^{-1}\right)_{m j}$, obternos:

$$
\left(\mathrm{S}^{-1}\right)_{i j}=\frac{1}{I_{3}}\left[\left(\mathrm{~S}^{2}\right)_{i j}-I_{1}(\mathrm{~S})_{i j}+I_{i \delta_{i j}}\right]
$$

sendo $\left(\mathbf{S}^{-1}\right)_{i j}(S)_{i j}=\delta_{i j}$,

Consideremos o tensor de segunda orden

$$
(\mathrm{S})_{i j}=a \delta_{i j}+b \varepsilon_{i j k} H_{i}+c H_{i} H_{i}
$$

onde $a, b$ a $c$ sầ coeficientes indeterminados. Das equaçoes (B.16), temos:

$$
I_{1}=3 a+c H^{2}, \quad I_{2}=3 a^{2}-\left(2 a c-b^{2}\right) H^{2} \text { e } I_{3}=\left(a^{2}+b^{2} H^{2}\right)\left(a+c H^{2}\right)
$$

Assim, da equação (B.17) podemos escrever que:

$$
\left(s^{-1}\right)_{i j}=\frac{1}{\left(a_{2}^{2}+b^{2} H^{2}\right)\left(a+c H^{2}\right)}\left[a\left(a+c H^{2}\right) \delta_{i j}-b\left(a+c H^{2}\right) \varepsilon_{i j} H_{b}+\left(b^{2}-a c\right) H_{i} H_{4}\right] \text {, }
$$

que é o tensor inverso para o tensor $(S)_{i j}$ dado pela equaçăto (B.18).

\section{B.4 Inversão de um Tensor de Quarta Ordem}

Consideremos o tensor de quarta ordem

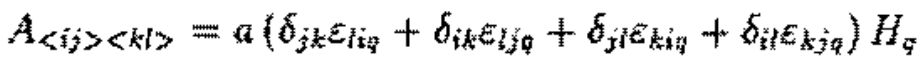

$$
\begin{aligned}
& +b\left(\delta_{i k} \delta_{j l}+\delta_{i j} \delta_{j k}-\frac{2}{3} \delta_{i j} \delta_{k i}\right) .
\end{aligned}
$$

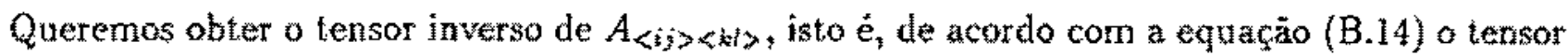
com componentes

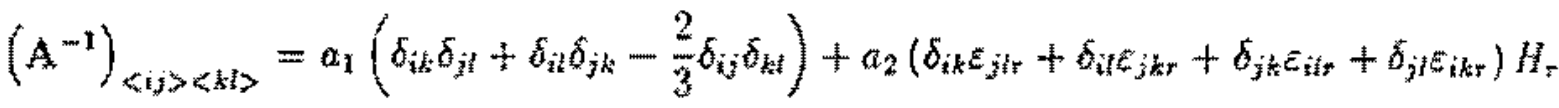

$$
\begin{aligned}
& +a_{3}\left(\delta_{i k} H_{j} H_{i}+\hat{\delta}_{i} H_{j} H_{k}+\delta_{j k} H_{i} H_{i}+\delta_{j i} H_{i} H_{k}-\frac{4}{3} \delta_{m i} H_{i} H_{j}-\frac{4}{3} \delta_{j j} H_{3} H_{1}+\frac{4}{9} H^{2} \delta_{i j} \delta_{k i}\right) \\
& +a_{4}\left(\varepsilon_{i k r} H_{j} H_{l}+\varepsilon_{i k r} H_{j} H_{k}+\varepsilon_{j k r} H_{i} H_{l}+\varepsilon_{j l r} H_{i} H_{k}\right) H_{r}
\end{aligned}
$$

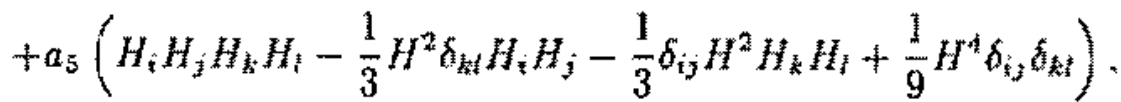

tal que

$$
\left(A^{-1}\right)_{\langle n\rangle\langle k j\rangle} A<k \mid>\langle m n\rangle=\frac{1}{2}\left(\delta_{m m} \delta_{m n}+\delta_{i n} \delta_{3 m}-\frac{2}{3} \delta_{i j} \delta_{m n}\right)
$$


onde o lado direito desta equaçäo representa os elementos da matriz identidade.

Substituindo as equaçoes (B.21) e (B.22) na (B.23), efetuando os produtos e rearranjando os termos, obtemos as seguintes relaçoes entre os coeficientes:

$$
\begin{aligned}
& a_{5} b-4 a_{4} a=0 \\
& a_{4} b-a_{3} a=0 \\
& 2 a_{4} a H^{2}+2 a_{3} b-3 a_{2} a=0 \\
& a_{2} b-a_{1} a=0 \\
& a_{1} b-4 a_{2} a H^{2}=\frac{1}{4}
\end{aligned}
$$

Resolvendo este sistema de equaços, obtemos:

$$
\begin{array}{ll}
a_{1}=\frac{b}{4\left(b^{2}+4 a^{2} H^{2}\right)}, & a_{2}=\frac{a}{4\left(b^{2}+4 a^{2} H^{2}\right)} \\
a_{3}=\frac{3 a^{2} b}{4\left(b^{2}+a^{2} H^{2}\right)\left(b^{2}+4 a^{2} H^{2}\right)}, & a_{4}=\frac{3 a^{3}}{4\left(b^{2}+4 a^{2} H^{2}\right)\left(b^{2}+4 a^{2} H^{2}\right)} \\
a_{5}=\frac{3 a^{4}}{b\left(b^{2}+a^{2} H^{2}\right)\left(b^{2}+4 a^{2} H^{2}\right)} &
\end{array}
$$

Com as equaçôes (B.25) temos para a equação (B.22)*

$$
\begin{aligned}
& \left(\mathrm{A}^{-1}\right)_{<i j\rangle\langle k\rangle}=\frac{1}{4\left(b^{2}+4 a^{2} H^{2}\right)}\left[b\left(\delta_{i k} \delta_{j l}+\delta_{i j} \delta_{j k}-\frac{2}{3} \delta_{i j} \delta_{k l}\right)\right. \\
& +a\left(\delta_{i k \varepsilon_{j l r}}+\delta_{i l} \epsilon_{j k r} \delta_{j k} \varepsilon_{i l \psi}+\delta_{j k} \epsilon_{i k r}\right) H_{r} \\
& +\frac{3 a^{2} b}{\left(b^{2}+a^{2} H^{2}\right)}\left(\delta_{i j} H_{j} H_{i}+\delta_{i l} H_{j} H_{k}+\delta_{j k} H_{i} H_{l}+\delta_{j} H_{i} H_{k}-\frac{4}{3} \delta_{k l} H_{i} H_{j}-\frac{4}{3} \delta_{i j} H_{k} H_{i}+\frac{4}{9} H^{2} \delta_{i j} \delta_{k l}\right) \\
& +\frac{3 a^{3}}{\left(b^{2}+a^{2} H^{2}\right)}\left(\varepsilon_{i k r} H_{j} H_{i}+\varepsilon_{i \xi} H_{j} H_{k}+\varepsilon_{j k r} H_{i} H_{t}+\varepsilon_{j i r} H_{i} H_{k}\right) H_{r} \\
& \left.+\frac{12 a^{4}}{b\left(b^{2}+a^{2} H^{2}\right)}\left(H_{i} H_{j} H_{k} H_{i}-\frac{1}{3} H^{2} \delta_{k l} H_{i} H_{j}-\frac{1}{3} H^{2} \delta_{i j} H_{k} H_{i}+\frac{1}{9} H^{4} \delta_{i j} \delta_{k t}\right)\right)
\end{aligned}
$$

que é o tensor inverso do tensor $A_{\langle i j\rangle\langle k\rangle}$ dado pela equaçă̌ $(B .21)$. 


\section{Apêndice $C$}

\section{Integração de uma Integral de Colisão}

Com a finalidade de llustrar o cálculo de uma integral de colisäo vamos calcular o lado direito da equaçăo (4.197) $\mathrm{com} \varphi=C_{<k} C_{i s}$, para obter o segundo membro da equação (4.201). Da equaçäo (4.198), temos:

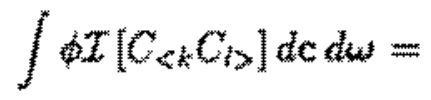

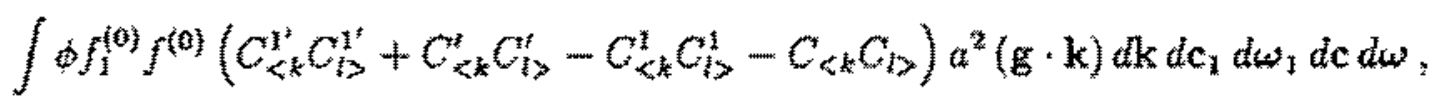

com o desvio dato pela equaça (4. 199$)$; dasde que os termos que contribuem para a integraça

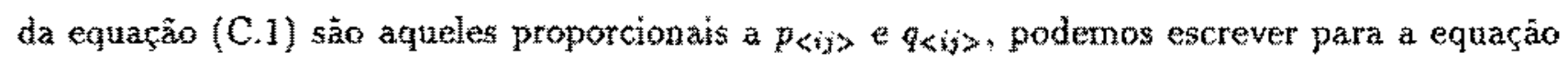
(1), 199):

$$
\phi=\frac{1}{2 Q}\left(\frac{m}{2 k T}\right)^{2} p_{\langle i j\rangle} C_{i} C_{j}+\frac{1}{2 m_{Q}}\left(\frac{m}{2 k T}\right)^{2} q_{<j\rangle} \Omega_{i} \Omega_{j}
$$

Corn a equação anterior escrevemos a (C.l) como $I=I_{1}+I_{2}$, onde

$$
I_{1}=\frac{1}{2 \varrho}\left(\frac{m}{k T}\right)^{2} p_{\langle i j\rangle} \int C_{i} C_{j} I\left[C_{<k} C_{l\rangle}\right] a^{2}(\mathrm{~g} \cdot \mathrm{k}) d \mathrm{k} d \mathrm{c}_{1} d \omega_{1} d \mathrm{c} d \omega
$$

e

$$
I_{2}=\frac{1}{2 m \varrho}\left(\frac{m}{k T}\right)^{2} q_{\langle i j\rangle} \int \Omega_{i} \Omega_{j} \mathcal{I}\left[C_{<k} C_{b}\right] a^{2}(\mathbf{g} \cdot \mathbf{k}) d \mathbf{k} d \mathbf{c}_{1} d \omega_{1} d \mathrm{c} d \omega
$$

Para a equação (C.3), temos:

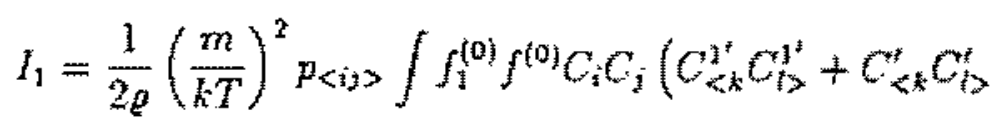

$$
\begin{aligned}
& \left.-C_{<k} C_{t>}^{1}-C_{<k} C_{D}\right) a^{2}(\mathrm{~g} \cdot \mathrm{k}) d \mathrm{k} d \mathrm{C}_{1} d \omega_{1} d \mathrm{c} d \omega
\end{aligned}
$$

E comveniante introduzimos as velocidades definidas por: 


$$
\begin{array}{ll}
g=c_{1}-c=C_{1}-C, & Q=\frac{1}{2}\left(C_{1}+C\right) \\
z=\omega_{1}-\omega=\Omega_{1}-\Omega, & Z=\frac{1}{3}\left(\Omega_{1}+\Omega\right)
\end{array}
$$

onde $\mathrm{g}$ e $\mathrm{z}$ săo, respectivamente, velocidades relativas linear e angular enquanto $G$ e $\mathrm{Z}$ são velocidades linear e angular do centro de massa, respectivamente. Corn as equaçōes (C.6) podemos mostrar que:

$$
d \mathrm{c} d \omega d \mathrm{c}_{1} d w_{1}=d \mathrm{~g} d \mathrm{~d} d \mathrm{G} d \mathbf{Z}
$$

e

$$
\begin{array}{ll}
\mathrm{C}=\mathrm{G}-\frac{1}{2} \mathrm{~g}, & \mathrm{C}_{1}=\mathrm{G}+\frac{1}{2} \mathrm{~g}, \\
\Omega=\mathrm{Z}-\frac{1}{2} \mathrm{z}, & \mathbf{\Omega}_{1}=\mathrm{Z}+\frac{1}{2} \mathrm{z},
\end{array}
$$

de modo que para as equaçōes $(1.28)$ a $(1.31)$, temos:

$$
\begin{aligned}
& C_{i}^{\prime}=G_{i}-\frac{1}{2} g_{i}+\frac{\kappa}{\kappa+1}\left[g_{i}-a \varepsilon_{i j k} k_{j} Z-k_{i}+\frac{1}{\kappa}(\mathrm{k} \cdot \mathrm{g})\right], \\
& C_{i}^{\prime \prime}=G_{i}+\frac{1}{2} g_{i}-\frac{k}{\kappa+1}\left[g_{i}-a \xi_{i j k} k_{j} Z-k_{i}+\frac{1}{k}(\mathrm{k} \cdot \mathrm{g})\right], \\
& \Omega_{i}^{i}=Z_{i}-\frac{1}{2} z_{i}-\frac{2}{a(1+\kappa)}\left(\varepsilon_{i j k} k_{j} g_{k}+a Z_{i}-a k_{i} k_{r} Z_{r}\right), \\
& \Omega_{i}^{\prime \prime}=Z_{i}+\frac{1}{2} z_{i}-\frac{2}{a(1+n)}\left(\varepsilon_{i j k} k_{j} g_{k}+a Z_{i}-a k_{i} k_{r} Z_{*}\right) .
\end{aligned}
$$

Também podemos escrever que

$$
\begin{gathered}
\mathscr{H}^{\mathrm{O})} f^{(\mathrm{v})}=\left(\frac{e}{m}\right)^{2} \frac{(m I)^{3}}{(2 \pi k T)^{6}} \exp \left[-\left(\frac{m C^{2}}{2 k T}+\frac{m \Omega^{2}}{2 k T}\right)\right] \\
=\left(\frac{e}{m}\right)^{2} \frac{(m l)^{3}}{(2 \pi k T)^{6}} \exp \left[-\left(\frac{m}{2 k T}\right)\left(2 G^{2}+\frac{1}{2} g^{2}\right)-\frac{1}{2 k T}\left(2 Z^{2}+\frac{1}{2} z^{2}\right)\right] \\
C_{i} C_{j}=G_{i} G_{j}-\frac{1}{2} g_{j} G_{i}-\frac{1}{2} g_{i} G_{j}+\frac{1}{4} g_{i} g_{j}
\end{gathered}
$$

e

$$
\begin{aligned}
& C_{\langle k}^{1} C_{1\rangle}^{\prime}+C_{<k}^{*} C_{1\rangle}^{\prime}-C_{<k}^{1} C_{1\rangle}^{\prime}-C_{<k} C_{1\rangle}=
\end{aligned}
$$

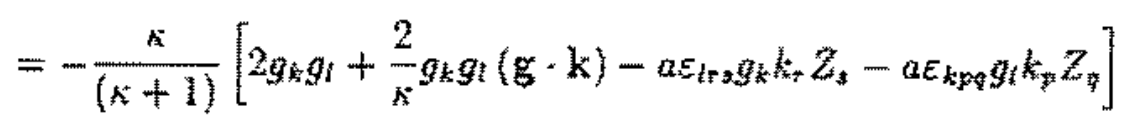

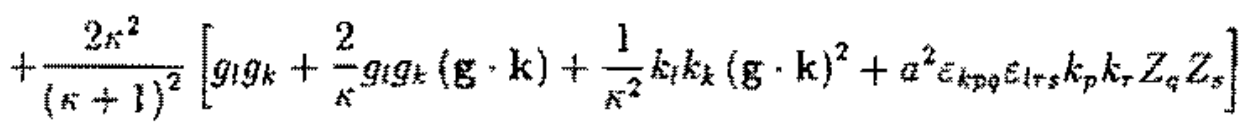




$$
\begin{aligned}
-\frac{2 \kappa^{2}}{3(k+1)^{2}}\left[g^{2}+\right. & \left.\frac{2}{\kappa}(\mathrm{g} \cdot \mathbf{k})^{2}+\frac{1}{\kappa^{2}}(\mathrm{~g} \cdot \mathbf{k})^{2}+a^{2} \varepsilon_{\mathrm{pgm}} \varepsilon_{\mathrm{pr} g} k_{\mathrm{g}} k_{\mathrm{F}} z_{m} z_{s}\right] \delta_{k \mathrm{i}} \\
& -\frac{2 \kappa}{3(\kappa+1)}\left[g^{2}+\frac{1}{k}(\mathrm{~g} \cdot \mathbf{k})^{2}\right] \delta_{k}
\end{aligned}
$$

Substituindo as equaços (C.7), (C.10), (C.11) e (C.12) na (C.5), obternos:

$$
\begin{aligned}
& \left.l_{1}=\frac{g}{2}\left(\frac{m I}{2 \pi}\right)^{3} \frac{p<i\rangle}{(k T)^{3}}\right] \exp \left[-\frac{m}{k T}\left(\alpha^{2}+\frac{1}{4} g^{2}\right)-\frac{I}{k T}\left(Z^{2}+\frac{1}{4} z^{2}\right)\right] \\
& {\left[G_{i} G_{j}-\frac{1}{2} g_{j} G_{i}-\frac{1}{2} g_{i} G-j+\frac{1}{4} g_{i} g_{j}\right]\left\{-\frac{x}{k+1}\left[2 g_{k} g_{l}+\frac{2}{k_{k}} g_{k} g_{l}(\mathrm{~g} \cdot \mathbf{k})\right.\right.}
\end{aligned}
$$

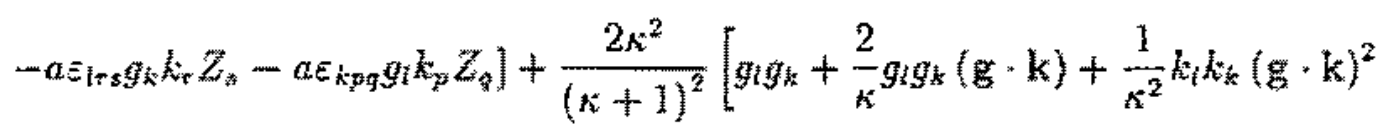

$$
\begin{aligned}
& \left.+a^{2} \varepsilon_{k p q} k_{k r s} k_{p} k_{s} k_{s} Z_{q} Z_{s}\right]-\frac{2 \kappa}{3(\kappa+1)}\left[g^{2}+\frac{1}{\kappa}(\mathrm{g} \cdot \mathrm{g})^{2}\right] \delta_{\mathrm{k} !}-\frac{2 \kappa^{2}}{3(\kappa+1)^{2}}\left[g^{2}+\frac{2}{\kappa}(\mathrm{g} \cdot \mathrm{k})^{2}\right.
\end{aligned}
$$

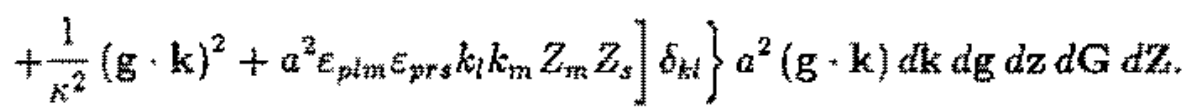

As integrais em $\mathbf{k}$ podem ser realizadas de imediato com as fórmulas do apêndice $\mathrm{A}$. Para realizar as integraçōes em $\mathrm{g}$ empregamos a transformaç̆o para coordenadas esfériè dg $=$ $4 \pi g^{2}$ tg as fórmulas do Apêndice $\mathrm{A}$; procedimento análogo e utilizado para as variáveis $G_{j}$, $z$ z. Ainda com o emprego das formulas do apendice A, obtemos para a equatäo (C.13):

$$
l_{1}=-\frac{8 \sqrt{\pi}}{15} g\left(\frac{a}{m}\right)^{2}\left(\frac{k T}{m}\right)^{1 / 2} \frac{(13 \pi+6)}{(k+1)^{2}} p_{<k l>}
$$

Com procedimento semelhante obtemos para a integraçäo da equação $(C .4)$

$$
l_{2}=-\frac{8 \sqrt{\pi}}{3} \varrho\left(\frac{a}{m}\right)^{2}\left(\frac{k T}{m}\right)^{1 / 2} \frac{k}{(k+1)^{2}} q_{<k l>}
$$

de modo que resulia para a equaçâo (C.2):

$$
I=-\frac{8 \sqrt{\pi}}{15}\left(\frac{a}{m}\right)^{2}\left(\frac{k T}{m}\right)^{1 / 2} \frac{1}{(k+1)^{2}}\left[(13 k+6) p_{\langle k l\rangle}+5 k a\langle t\rangle\right]
$$

que o lado direito da equaço $(4.201)$. 


\section{Apêndice D}

\section{Polinômios de Sonine}

Certos problemas em fisica teórica, como por exemplo, o problema de autovalores na mecânica quântica, recaem em equaçồs diferenciais do tipo

$$
x y^{3 *}+(m+1-x) y^{\prime}+(n-m) y=0
$$

que é conhecida como equaça diferencial associada de Laguerre. As soluçôes desta equaçäo para valores năo negativos de $m$ e $n$ são dadas através dos polinômios associados de Laguerre de gratu $n-m$

$$
y(x)=L_{n}^{\text {m) }}(x), \quad \text { para } \quad m \leq n
$$

A função geratiz dos polinônios $L_{n}^{(m)}(x)$ é dada por

$$
(-1)^{m}(1-z)^{m(m+n)} \exp \left(-\frac{x z}{1-z}\right)=\sum_{n=0}^{\infty} \frac{L_{n+m}^{(m)}(x)}{(n+m) !} z^{n}
$$

enquanto que suán fómula geral é

$$
L_{n}^{(m)}(x)=\sum_{k=0}^{n-m n}(-1)^{k+n} \frac{(n))^{k}}{(n-m-k) !(k+m) !} \frac{x^{k}}{k !}
$$

Os polinomios de Laguerre $L_{r h}^{(m)}(x)$ formam um conjunto de funçós ortogonais com relaçào à funça peso $z^{-7} x^{m}$ no intervalo $0<x<\infty$. isto é,

$$
\int_{0}^{\infty} e^{-x} x^{m} L_{n}^{(m)}(x) L_{p}^{(m)}(r) d x=\frac{(n !)^{3}}{(n-m) !} \delta_{n y}
$$

Conforme é visto no capítulo 5 , a solusäo da equação de Boltzmann através do método de Chapman-Enshog requer a expansăo de coeficientes em série de polinônios de Sonine $S_{m}^{(n)}(x)$. Estes estâto relacionados com os polinómios associados de Laguerre alravés de 


$$
S_{m}^{(n)}(x)=\frac{(-1)^{m}}{(n+m) !} L_{n+m}^{(m)}(x)
$$

Portanto, segundo as equaçōes (D.3) e (D.6), a funçäo geratriz, a fórmula geral e a condiçăo de ortogonalidade para os polinômios de Sonine são expressas, respectivamente, por

$$
\begin{gathered}
(1-z)^{-(m+1)} \exp \left(-\frac{2 z^{z}}{1-z}\right)=\sum_{n=0}^{\infty} S_{m}^{(n)}(x) z^{n}, \\
S_{m}^{(n)}(x)=\sum_{k=0}^{n} \frac{(n+m) !}{k !(n-k) !(k+m) !}(-x)^{k}, \\
\int_{0}^{\infty} e^{-x} x^{m} S_{m k}^{(n)}(x) S_{m i}^{(p)}(x) d x=\frac{(n+m) !}{n !} \delta_{n p} .
\end{gathered}
$$

Para os polinômios de Sonine de ordem $n$, indice $\left(l+\frac{1}{2}\right)$ e variável $\beta C^{2}=\frac{m C^{2}}{2 k T}$, as equaçōes (D.8) e (D.9) säo escritas na forma

$$
\begin{aligned}
& S_{i+\frac{1}{2}}^{(n)}\left(\beta C^{2}\right)=\sum_{k=0}^{n} \frac{\Gamma(n+l+3 / 2)}{k !(n-k) ! \Gamma(k+1+3 / 2)}\left(-\beta C^{2}\right)^{k},
\end{aligned}
$$

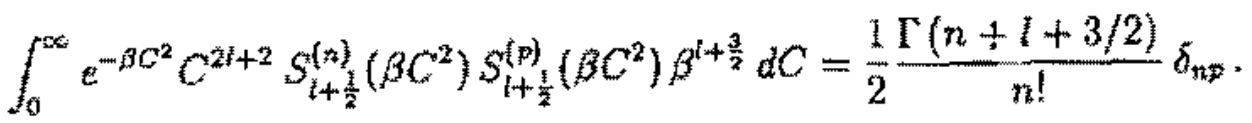

onde $\Gamma\left(m+\frac{1}{2}\right)=\frac{(2 m-1) !}{2^{m}} \sqrt{\pi}$ é a função gama.

A partir da equação (D.10) escrevemos os três primeiros polinổnios de Sonine:

$$
\begin{gathered}
S_{i+\frac{1}{2}}^{(0)}\left(\beta C^{2}\right)=1 \\
S_{1+\frac{1}{2}}^{(1)}\left(\beta C^{2}\right)=l+\frac{3}{2}-\beta C^{2} \\
S_{i+\frac{1}{2}}^{(2)}\left(\beta C^{2}\right)=\frac{1}{2}\left(l+\frac{5}{2}\right)\left(l+\frac{3}{2}\right)-\left(l+\frac{5}{2}\right) \beta C^{2}+\frac{1}{2} \beta^{3} C^{4}
\end{gathered}
$$




\section{Apêndice $\mathbf{E}$}

\section{Condição de Salto para Campos}

Se $\Psi$ representa uma propriedade aditiva qualquer e $\psi$ a sua densidade, a quantidade $\psi_{4}$ um volume material $V(t)$, cuja superfície $\partial V(t)$ se move com uma velocidade $v$ igual a velocidade dos pontos materiais interiores à superície $\partial V(t)$, é dada por

$$
\Psi v=\int_{V(t)} \psi d V
$$

A mudança temporal $\Psi_{V}$ terá, em geral, duas contribuições ${ }^{1}$ :

(1) uma contribuicão devida a uma densidade de fluxo $\varphi_{\text {i }}$ através da superficie $\partial V(t)$. Se $\mathbf{n}$ representar a normal unitária superficie $\partial V(t)$ e da um elemento de àrea desta superficis, 0 fluxo através da superficie será dato por:

$$
-\oint_{a v(t)} \Phi_{i} n_{i} d a
$$

(ii) uma contribuição devida a uma densidade de produção a no interior do volume V(i), isto é.

$$
\int_{V[t]} d V
$$

Portanto, a mudança temporal de $\Psi$ v pode ser escrita como

$$
\frac{d}{d t} \int_{V(t)} \psi d V=-\int_{\partial V_{(t)}} \Phi_{i} \eta_{i} d d t+\int_{V(t)} d d V
$$

O seguinte teorema é vălido para volumes materiais:

Teorema de tranporte: Seja $V(t)$ um volume material cuja superfície $\partial V(i)$, de normal unitána n. se move com velocidade $v$. Entäo, para qualquer função suave $\psi(x, y)$, temos [mo]:

$$
\frac{d}{d t} \int_{y(t)} \psi d V=\int_{v(t)} \frac{\partial \Psi}{\partial t} d V+\int_{\partial v(t)} u v \cdot n d a
$$

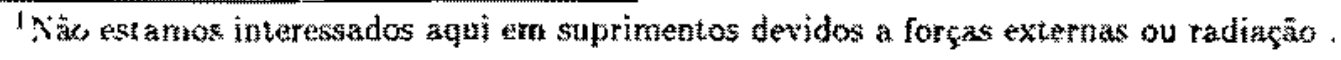




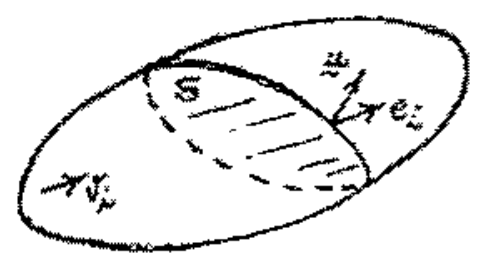

Figura E.1: Superficie singular para um volume de fluido

Se combinarmos as equaços $($ E. 4$)$ e $($ E.5) e utilizarmos o teorema da divergência segue que

$$
\int_{v(t)}\left[\frac{\partial \psi}{\partial t}+\frac{\partial}{\partial x_{i}}\left(\psi w_{i}+\psi_{i}\right)-\partial\right] d y=0
$$

Como a equação anterior é válida para todos os pontos regulares do volume material $V(t)$. obtemos a forma local da equaçăo geral de balanço

$$
\frac{\partial \psi}{\partial t}+\frac{\partial}{\partial x_{i}}\left(\psi+w_{i}\right)=\sigma
$$

Dizemos que $S(i)$ é uma superficie singular se algum campo experimenta um salto (ou descontinuidade) atraves de $S$. Considere agora que o volume material $V(t)$ contem uma superficie singular $S(t)$. Sendo $\phi$ um campo seus respectivos limites $\phi^{*}$ e $\phi^{-2}$ en ambos os lados de $S$ existem mas não säo necessáriamente iguais. Define-se o salto do campo $\phi$ através de $S$, por

$$
\llbracket \phi=\phi^{*}-\phi^{*}
$$

Se a superficie singular $S$ se desloca com uma velocidade $t_{i}(x, t)$, onde $\varepsilon_{i}$ um versor nomal à superície singular (veja a figura E. I), a forma geral da equaça de balanço em pontos singulares é dada por $[69]$

$$
\llbracket \Phi_{i} \rrbracket e_{i}+\| \psi_{1}\left(u_{i}-u_{i}\right) \rrbracket e_{i}=0
$$

Na tabela 1 apresentamos as respectivas densidades, fluxos e produçoes ao identificarmos a trandeza $\Psi$ a massa, momento linear, energia e entropia: $p_{i j}$ é tensor pressao, $q_{j}$ o fuso de

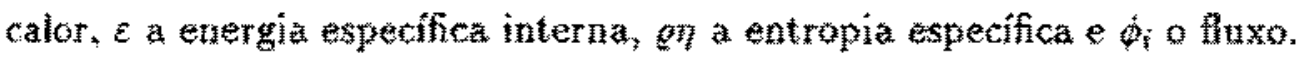

Das equaçóes (E.7) e (E.9) resultam as seguintes equaçöes de balanço local e para pontos singulares do fluido: 


\begin{tabular}{|l|c|c|c|}
\hline & $\psi$ & $\Phi_{i}$ & $\sigma$ \\
\hline massa & $\varrho$ & 0 & 0 \\
\hline momento linear & $\varrho \nu_{i}$ & $p_{i j}$ & 0 \\
\hline energia & $\varrho\left(c+\frac{1}{2} v^{2}\right)$ & $p_{i j} z_{i}+q_{i}$ & 0 \\
\hline entropia & $\varrho \eta$ & $\phi_{i}$ & $\varsigma$ \\
\hline
\end{tabular}

Tabela E.1: Densidades, fluxos e produçoes para a grandeza $\Psi$

- massa

$$
\begin{aligned}
& \frac{\partial g}{\partial t}+\frac{\partial g x_{i}}{\partial x_{i}}=0 \\
& \llbracket g\left(w_{i}-w_{i}\right) \rrbracket e_{i}=0
\end{aligned}
$$

- densidade de momento linear

$$
\begin{aligned}
& \frac{\partial g v_{i}}{\partial t}+\frac{\partial}{\partial x_{j}}\left(2 v_{i} v_{j}+p_{i j}\right)=0 \\
& u p_{i j} \rrbracket e_{j}+\llbracket p v_{i}\left(v_{j}-u_{j}\right) \rrbracket e_{j}=0
\end{aligned}
$$

- energia especifica

$$
\begin{aligned}
& \frac{\partial}{\partial t}\left[\varrho\left(\varepsilon+\frac{1}{2} v^{2}\right)\right]+\frac{\partial}{\partial x_{j}}\left[\varrho\left(\varepsilon+\frac{1}{2} v^{2}\right) v_{j}+p_{i j} v_{i}+q_{j}\right]=0 \\
& \left.\left\|g_{j}+p_{i j} v_{i}\right\| e_{j}+\| e\left(\varepsilon+\frac{1}{2} v^{2}\right)\left(v_{j}-u_{j}\right)\right] \epsilon_{j}=0
\end{aligned}
$$

- densidade de entropia

$$
\begin{aligned}
& \frac{\partial \eta \eta}{\partial t}+\frac{\partial}{\partial x_{i}}\left(\omega \eta v_{i}+\phi_{i}\right)=\zeta \geq 0 \\
& \llbracket \phi_{i} \rrbracket e_{i}+\llbracket \varrho \eta\left(w_{i}-w_{i}\right) \| e_{i}=0
\end{aligned}
$$

No capítulo 5 utilizamos as condiçöes de salto para um volume rnaterial com uma superficie singular ambos em repouso $\left(v_{i}=0\right.$ e $\left.w_{i}=0\right)$, de modo que as condiçoes de salto A.15 e A.IT podem se escritas como

$$
\begin{aligned}
& \left\|q_{i}\right\| \varepsilon_{i}=0 \\
& \left\|\phi_{i}\right\| \varepsilon_{i}=0
\end{aligned}
$$




\section{Bibliografia}

[1] Maxwell, J.C. Mustrations of the Dynamical Theory of Gases, Phil. Magarine 19 (1860) 19-32; $20(1860) 21-36$.

(2) Maxwell, J.C.: On the Dynamical Theory of Gases, Phil. Trans. Roy. Soc. London 157 (1867) 4988

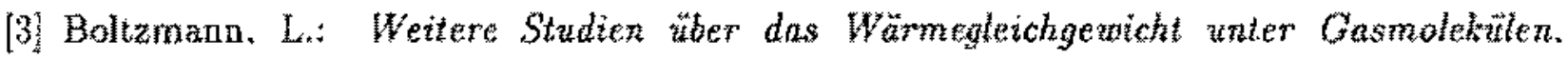
Sitzungs Berichte, Kaiserl. Akad. der Wissenschaften 60 (2) (1872) 275-370.

[4] Boltzmann, I.: Ueber das Wärmegleichgewicht won Gasen, auf welch äussere hrafite wirken, Sitzungs Berichte der Oester. Akad. der Wissenschalten, Mathem.-Naturwiss. Klasse t2 (2) (1875) 427-4t5.

[5] Lorentz, H.A.: Ueber das Gleichgevicht der lebendigen Kraft unter Gasmoletwlen, Sitz. Ber. Kaiserl. Akad. der Wiss. Mathem.-Naturwiss. Klasse 95 (1887) 115 152.

[6] Hibert, D.: Begründung der kinetischen Gastheoric, Mathematische Annalen 72 $(1916 / 17) 562-577$.

[7] Chapman, S.: The Kinetic Theory of Simple ard Composite Gases: Viscosity; Thermal Conduction and Diffusion. Proc. Roy. Soc. Lond. A93 (1916/17) 1-20.

[8] Enskog, D.: Kinetische Theorie der Vorgänge in mässing verdünnten Gasen, Almquist and Wiksell, Uppsala (1917).

[9] Chapman, S.; Cowling. T.G.: The Mathematical Theory of Non-Uniform Gases. Cambridge: Cambridge Univ. Press $(1970)$.

110j Burnett, D.: The Distribution of Molecular Velocities and the Mean Motion in a NonLwowm Gas, Proc. London Math. Soc. 40 (1935) 382.435.

111] Giad, H.: On the Kinctic Theory of fordfed gascs, Commun. Pure Appl. Math. 2 (1949) $31.40 \%$ 
[12) Wang-Chang, C.S.; Uhlenbeck, G.E.; de Boer, J.: The Heat Conducivity and Viscasty of Polyatomic Gases, Studies in Statistical Mechanics, Vol.1, North-Holland, Amsterdan (1964) $243 \cdot 268$

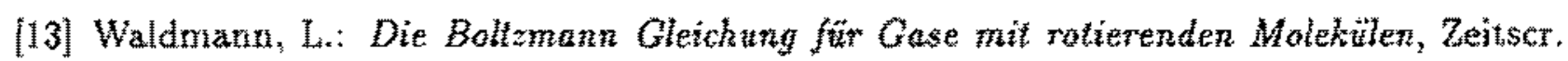
Nacurt. 12a (1957) 660-662.

[14] Snider, R.F. Quantum-Mechanical Modffed Bollzman Equation for Degenerate Internal States, J. Chem. Phys. 32 (1960) 1051.1060.

[15] McCourt, F.R.W.; Snider, R.F.: Thermal Conductiuity of a Gas with Ratational States, J. Chem. Phys. 41 (1964) 3185-3194; 43 (1955) 2276.2283.

[16] McCourt, F.R.W.; Beenakker, J.J.: Magnetic and Eletric Efects on Transport Properties, Ann. Rev. Phys. Chem. 21 (1970) 47-72.

[17] Bryan, G.H: On our Knowledge of Thermodynamacs, Brit. Assoc. Reports (1894) 83.

[18] Bezerra Jr, A. G.: Reinedke, S.; Kremer, G: A Combined Chapmam-Enskog and Grad Method. H.Monatomic Gases and Mirines, Continum Mech. Thermodyn. 6 (1994) 149 160.

[19] Rodbard. M. G.; Bezerra Jr., A. G.; Kremer, G.M.: A Combined Chapman-Enskog and Grad Method. II.Ion:zed Gases, Physics of of Plasmas, 2, 642 (1995).

[20] Kagan, Yu.: Afanase'v, A. M.: On the Kinetic Theory of Gases with Rotational Degrees of Freedom, Sov. Phys. JETP, 14 (1962) 1096-1101.

[21] Smith, G. F.: On the Ssotropic Function of Symetric Tensors, Screw-Symetric Tensors and Vectors. J. Int. Engien. Sci., 2 (1971) 899-916.

[22] Maxwell, J.C.: On Stresses havefued Gases Arising from Inequalities of Temperalure. Phyl. Trans. Roy. Soc. 170 (1879) $231-256$.

[23] Brush, S.G.: A History of the Kinetic Theory of Gases in the 19th Century. Vol.1: Physics and the Atomists. North-Holland, Amsterdam (1986).

124) Scott, G.G.; Sturner, H.W. Willianson, R.M.: Gas Torque Anomaly in Weak Magnelic Ficlds. Phys. Rev. 158 (1967) $117+121$.

[25] Levi, A.C.: Beenakker, J.J.M.: Thermomagnetic Torques in Dilute Gases. Phys. Letters $25 \mathrm{~A}(1965) 350-352$. 
[26] Kremer, G.M.: Extended Thermodyamics of Molecular Ideal Gases, Continuum Mech. Thermodym. 1 (1989) 21-45.

[27] Kremer, G.M: Zut entetterten Themodynamik idealer thd dichter Gase. Diss. TU Berlin $(1085)$

[28] Ikenberry, E*: Truesdel, $C_{*}:$ On the Pressure and the Fiv po Energy in a Gas According to Maxwell's Kinetic Theory I, J. Ration. Mech. Anal. 5 (1956) 1-54.

[29] Ferziger, J. H.; Kaper, H. G.: Mathematical Theory of Transport Processes in Gases, North Holland, Amsterdam (1967).

[30] Kogan, M. N.: Rarefied Gas Dynambs, Plenum Press, New York (1969).

[31] Kremer, G. M.:A Equaçāo de Boltzmann os Processos de Transporte em Gases notas de aula para o curso de Pós-Graduação em Física, Dep. de Física, UFPR, Curitiba (1995).

[32] Kremer, G. M.: Teoria Cinêtica de Gases Poliatomicos, Tese apresentada para o Concurso de Professor Titular, Dep. de Fisica, UFPR, Curitiba (1987)

[33] Sommertald, A.: Temodynamics and Statiscal Mechanics. Lectures on Theorefical Phasics, vol. V, p. 332. New York: Academic Press, Inc. (1967).

[34] Lax, E.: Taschenbuch für Chemiker und Physiker, vol. 1, Springer-Verlag, Berlin, Heidelberg, New York (196\%).

[35] Bousheri. A. J* Bzowski, J* Kestin, J. e Mason, E. A.: Equibribm and Transport Properties of Eleven Polyatomic Gases at Low Densiby, J. Phys. Chem. Ref. Data, 16 . 3. $(1987) 445-466$.

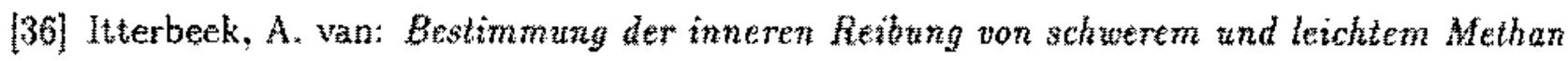
zwischen ges $K$ und $90 \mathrm{~K}$, Physica, 2.831 (1940).

[37] Reid, R. C.; Sherwood, T. K.: The Properties of Gasts and Liqutds, Mc Graw Hill, New York $(1966)$.

[38] Eucken. E.: Veber das Warmeituermogen, dic spezifsche Wäme und die innere Reitung dev Gase, Phys. Zeits. 14 (1913) 324,332.

[3ol Pidduck. F.B.: The Kinetic Theory of Specint Type of a Rigit Molecules, Proc. Roy. Soc. A. $101(1922) 101-112$. 
[40] Jeans, J. H.: The Distribution of Molecular Energy, Phil. Trans. Roy. Soc, A196 (1901) $397-431$.

[41] Kihiara, T: J. Phys. Soc, Japan, 6 (1951) 289.

[42] Cercignani, C.; lampis, M.: On the H-theorem for Polyatomic Gases, J. Stat. Phys., 26 (1981) $795 \cdot 801$.

[43] Reinecke, 9.; Kremer, G.: A Generalization of the Chapmen-Enskon and Orat Menods. Continuum Mech. Thermodyn., 3 (1991) 155-167.

[44] Kremer, G.M.: Kinetic Theory of a Rarefied of Rough Spheres, Rev, Bras. Fis. 17 (1987) $370-386$.

[45] Senfleben, H.: Einfluss eines Magnetfelds auf das Wärmleitungsvermögen von paramagnetischen Cusen, Physik. Zeitschr., 31 (1930) 961-963.

[46] Engelhard, H.; Sack, H.: Physik, Zeitschr, 33 (1932) 724-727.

[47] Gorter, C. J.: Zur Interpretierong des Senfleben-Effekts, Naturwista 26 (1938) 140.

[48] Beenakker, J. J. M.; Scoles, G.; Knaap, H. F. P.: Jonkman, R. M.: Physs. Lett., 2. (1962) $3-6$.

[49] Wakeham, W. A; Dickinson, A. S.; McCourt, F. R. W.; Vesovic, V.: Status and Future Deatopments in the Study of Transport Properties, NATO ASI Séries. Kluwer Academic Publis., Dordrecht, Boston, London (1992) $117-153$.

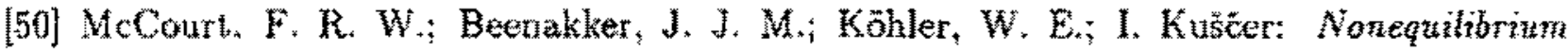
Phenomena in Polyatomic Gases. Vol. 1: Dilute Gases, Oxford University Press, Oxford (1990).

[51] Kagan, Yu.; Maksimov L.: Transport Phenomena in Paramagnetic Gas, Sov. Phys. JETP, 14. (1952) 604-610.

[52] Knaap, H. F. P.: Beenakker, J. J. M.: Heat Conducizuty and Viscosity of a Gas of

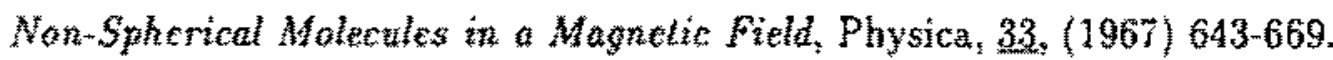

[53] Klein, M. W.: Hofman, D. K.: Dahler, J. S.: Extemal Futu Dependenef of Tratsport Propertics. 1. Thermal Conduction in a Fiad of Rough Spewes. J. Chem. Phys. 49 (19to) $2321-2333$. 
[54] Hulsman, H.; Knaap, H. F. P.: Experimental Arrangements for Measuring ble Fve Independent Shear. Viscosity Coefficients in a Polyatomic Gas in a Magnetic Field. Physica. $50(1970) 565-572$.

[55] McCourt, F. R. W. Knaap, H. F. P. Moraal, H.: The Senfleben-Beenakker Eftects for a Gas of Rough Spheres Spherical Molecules, 1. Themal Contuctinty, Physica, 13 (1969) $485-512$.

[56] Moraal, H.; McCourt, F. R.; Knaap, H. F. P.: The Senftleben-Beenakker Effects for a Gas of Rough Spherical Molecules. II. The Viscosity Scheme, Physica, 45 (1969) 4554668.

[57] Kogan. M.N. On the Principle of Maximum Entropy, Proc. $5^{\text {th }}$ Int. Symp. Rar. Gas Dyn., vol. I, Academic Press, New York (1967) 359-368.

[58] Butkov, E.: Mathematical Physics, Reading, Mass.: Addison-Wesley, 1973 (Capitulo 13)

[59] Waldmann, L: Proc. Int. Sem. on the Transp. Prop. of Gases. Providence (1964) 59.

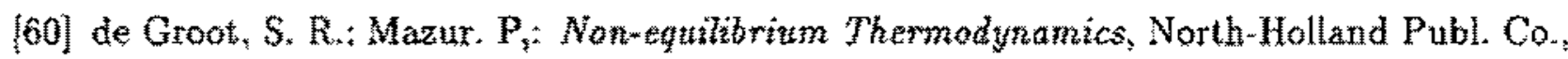
Amslerdam (1969).

[61] Condiff, D. W.; Lu, W. K.; Dahler,J. S.: Trasporl Properties of Polyatomic Futids, a Dilute Gas Of Perfectly Rough Spheres, J. Chem. Phys, 42 (1965) 3445-3475.

[62] Korving, J, Honeywell, W. I, Bose T. K.; Beenakker, J. J. M.: The Influence of Magnetic Futd an the Transport Properties of Cases of Polyatomic Molecules. II. Thermal Conducturity, Physica, 36 (1967) 198-214.

[63] Hermans, L. J. F.; Fortuin, P. H.; Knaap, H. F. P.; Beenakker, J. J. M.: Transwerse Htat Transport in Polyatomic Gases under Infiuence of a Magnetic Field, Phys. Letters. $25 \mathrm{~A}$. $2(195 \%) 81-82$.

[6.1] Thijsse, B. J.; Denissen, W. A. P, Hermans, L. J. F; Knaap, H. F. P.; Beenakher, J. J. M.: The Thernal Conductivity of Polar Gases in a Magnetic Field, Physica, 9f A (1979) $467+514$.

[65] Hulsman, H.; Burgmans. A.L.J.: The Five Shear Viscosity Coefficients of a Polyatomic Gas in a Magnetic Fitd. Phys. letters, 29A (1969) 629-630.

[60] Fock. J.; Uhlenbeck, G.E. Propagation of Sound in Monatomic Gases. Phys. Rev Letters $19(1967) 1025,1027$. 
[67] Marques Jr, W; Kremer, G.M.: On Enskog's Dense Gas Theory. II. The Linearivex Burnett Equations for Monatomic Gases, Rev. Bras. Fis., 21 (1991) 402 417 .

[68] Fervandes, A.S,; Kremer, G. M.: Kinetic and Phenomenological Theories for the Linearized Burnett Equations of a Molecular Gas, Continuum Mech. Thermodyn., 5 (1993) $67-81$.

[69] Müller, 1.: Thermodynamics, Pitman Advanced Publishing Program, Boston, Loddon, Melbourne (1985).

[70] Liu, I-Shih: Mechanies of Continuous Metra. Notas de Aula, IM-UFRu (1995). 